\title{
A Study of the Sorption Characteristics of Nanostructured Calcium Silicate
}

\author{
by \\ Mathew James Cairns \\ Victoria \\ Te Whare Wānanga \\ o te Ūpoko o te Ika a Māui

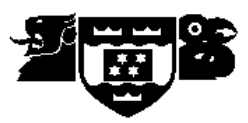

A thesis

submitted to Victoria University of Wellington in fulfilment of the requirements for the degree of

Doctor of Philosophy in Chemistry.

Victoria University of Wellington 2008 


\begin{abstract}
Nanostructured calcium silicate (NCS) is an X-ray amorphous silicate material consisting of randomly arranged platelets several tens of nanometres in size, forming agglomerates a few micrometres in size. This affords the material a high, readily accessible surface area of up to $600 \mathrm{~m}^{2} \mathrm{~g}^{-1}$ with chemically active surface-bound calcium and silanol groups being integral parts of the silicate structure. As such, it makes an ideal material for the sorption of many potential pollutant materials. However, NCS is highly thixotropic. This reduces its applicability for use as a sorbent material on a large scale, the thixotropic nature of NCS precluding its efficient separation from suspension.
\end{abstract}

NCS, in contact with water, will ion-exchange surface-bound calcium with hydrogen ions, releasing calcium into solution, and leading to an increase in the $\mathrm{pH}$ value of the solution. The process may be exploited by using the material as a sorbent for cationic metal species forming insoluble hydroxides. This thesis demonstrates the use of NCS as a sorbent material for $\mathrm{Cu}^{2+}$, with the material exhibiting a sorption capacity for this ion of up to $10 \mathrm{mmol} \mathrm{g}$. When the sorption capacity of the material is reached, all the calcium initially present in the NCS material $(31-38 \mathrm{wt} \% \mathrm{CaO})$ is leached into solution. The copper is initially sorbed as an X-ray amorphous phase (most likely $\left.\mathrm{Cu}(\mathrm{OH})_{2}\right)$ but in the presence of excess copper, the more thermodynamically stable crystalline phase $\mathrm{Cu}_{2} \mathrm{X}(\mathrm{OH})_{3}, \mathrm{X}$ being chloride or nitrate, is formed. It was shown that the presence of calcium is necessary for this sorption to occur. When calcium was leached from the material prior to sorption studies, the sorption capacity of the material was significantly decreased.

To aid the separation process of NCS from solution, bulk magnetite powder $\left(\mathrm{Fe}_{3} \mathrm{O}_{4}\right)$, or superparamagnetic magnetite or maghemite $\left(\gamma-\mathrm{Fe}_{2} \mathrm{O}_{3}\right)$ were incorporated into the NCS structure during its synthesis. The addition of these additives to the NCS material reduced characteristics such as specific surface area or sorption capacity insofar as extra mass had been added to the system. The structure of the NCS was not degraded. The NCS material containing bulk magnetite powder was shown to be applicable to the sorption of phosphate in a continuous fluidised bed system, utilising the magnetic properties of the material to 
aid separation. Phosphate was chosen, as the sorption characteristics of NCS with respect to this ion were previously known.

Attempts to use magnetic techniques to separate the superparamagnetic composites subsequent to copper sorption were unsuccessful. Although the composite materials exhibited similar sorption capacity for copper to the unmodified one, the acidic conditions of the copper solution degraded the composite, precluding the use of magnetic separation.

Finally, composite materials of NCS and a conducting polymer, polyaniline, were prepared which provided potential redox-activity to a high surface area substrate. The sorption characteristics of this material were demonstrated with its use as a sorbent for the perrhenate ion. This rhenium ion was chosen due to its chemical similarity to pertechnetate, a component of many radioactive wastewaters. It was demonstrated that the sorption process proceeded via an electrochemical mechanism in which the polyaniline caused the perrhenate ion to be reduced to a rhenium oxide species. 


\section{Acknowledgements}

There are a great many people who should be acknowledged for their help and support with this project. As is often the case with a work of such size, a name will occasionally be overlooked. Any omissions in the list below are entirely accidental. If you have assisted me in some small way with this project, I offer you my gratitude.

Above all else, I would like to thank my supervisor, Thomas Borrmann. His guidance and support with this project has been invaluable and far exceeds what one could reasonably expect. I offer him my most sincere thanks. Jim Johnston, as my co-supervisor also deserves my deepest thanks. It has been a pleasure to work with you both.

Special thanks are also due to Andy McFarlane. His knowledge of silicates and willingness to answer questions was incredibly helpful in the early stages of this project. His sense of humour has also enlivened many an otherwise dull evening in the lab.

Over the years, our lab has seen a lot of people come and go. I'd like to express my gratitude to the past and present members of materials chemistry research group - Aaron, Fern, Kersitn, Mike, Amy, Jess, Carla, James, Daniel, Tobias, Daniela, Andi, Ramprasad, Christian, Kathrin, and many, many others who've passed through the lab over the years. Without you all, the lab just wouldn't be the same.

One Tuesday evening several years ago, I was dragged out of the lab and introduced to the social life of the department. For that, I have to thank Laine and Rhys. Although the Tuesday social group is no longer, thanks also go to John, Kirsten, Janeen, Jonathan, Chris, Scotty and the other regulars at more recent social events for the friendship they've shown.

I'd like to thank Jackie, Teresa, Gordon, Alan and Manu for their technical help, especially getting pieces of equipment fixed, or getting access to it on short notice.

Writing can, at times, be a very frustrating process with the possibility to effect those around you. Because of this, I want to thank Wendy, Jo, Sarah, Kathryn, Teresa, Ying, Hui Lin, Brad, and Almas, the people with whom I've shared an office, for putting up with me over the last several months.

The congenial atmosphere of the department was integral to the success of this project. I wish to thank the innumerable people who contributed to this in some small way.

Part of the work described herein was undertaken using the facilities of the Institute for Technical Chemistry at the Forschungszentrum Karlsruhe, Germany, with funding from the German Research Foundation. I want to express my gratitude to Wolfgang Höll for making that research possible. I'd also like to thank the many people at that institute who welcomed me with open arms — Julia, Carla, Dirk, Marcel, Marcelo, José, and Asma, to name but a few.

Last, but by no means least, is my family. I am deeply appreciative of the unconditional support and understanding that my parents Annette and Eric, and my sister Joanna, have provided over the course of this project. None of this would have been possible without you. 


\section{Table of Contents}

$\begin{array}{lll}\text { Abstract } & \text { ii }\end{array}$

Acknowledgments $\quad$ iv

Table of Contents $\quad$ v

List of Figures $\quad$ ix

List of Tables $\quad$ xiii

$\begin{array}{ll}\text { Publications } & \text { xv }\end{array}$

$\begin{array}{ll}\text { Glossary } & \text { xvi }\end{array}$

1 Introduction 1

1.1 Water Pollution and the Environment . . . . . . . . . . . . . . . 1

1.1.1 Water Quality . . . . . . . . . . . . . 2

1.2 Sorption Models . . . . . . . . . . . . . . . . . . . 5

1.2.1 Sorption Isotherms _. . . . . . . . . . . . . . . 5

1.2.2 Direct Fitting Models . . . . . . . . . . . . . . . . . 9

1.2.3 Diffusion-Controlled Sorption . . . . . . . . . . . . . . 11

1.2.4 Kinetics of Sorption . . . . . . . . . . . . . . . . . 13

1.3 Nanostructured Calcium Silicate . . . . . . . . . . . . . . . . 15

$1.3 .1 \quad$ Structure .......................... 16

1.3.2 Physical Properties . . . . . . . . . . . . . . . . . 20

1.3.3 Chemistry ....................... 20

1.3.4 Conducting Polymer Derivatives . . . . . . . . . . . . . 21

1.4 Scope of the Thesis . . . . . . . . . . . . . . . . . 22 


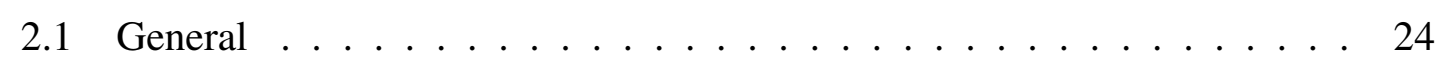

2.1.1 Chemical Analytical Techniques . . . . . . . . . . . 25

2.1.2 Other Instrumental Techniques . . . . . . . . . . . . . 30

2.2 Synthesis of Calcium Silicate and Its Derivatives _ . . . . . . . . . . . 34

2.2.1 General Synthetic Procedure . . . . . . . . . . . . . 34

2.2.2 Synthesis of Magnetic Calcium Silicate Composites . . . . . . 37

2.2.3 Synthesis of Calcium Silicate-Polyaniline Composites . . . . . . 39

2.3 Sorption Studies of Copper . . . . . . . . . . . . . . . . . 41

$2.3 .1 \quad$ Kinetic Studies . . . . . . . . . . . . . . . . . 41

2.3.2 Isotherm Measurements . . . . . . . . . . . . . . . 42

2.4 Sorption Studies of Phosphate . . . . . . . . . . . . . . . 42

2.4 .1 Isotherm Studies $\ldots \ldots$. . . . . . . . . . . . . . 42

2.4.2 Kinetic Studies . . . . . . . . . . . . . . . 43

2.4.3 Continuous Uptake Studies . . . . . . . . . . . . . . . . . . . 43

2.5 Sorption Studies of Rhenium . . . . . . . . . . . . . . . 45

2.5.1 Sorption by Polyaniline Films . . . . . . . . . . . . . 45

2.5.2 Sorption by PANI-NCS Composites . . . . . . . . . . . . 46

3 Copper Sorption $\quad 48$

3.1 Introduction . . . . . . . . . . . . . . . 48

3.1 .1 Heavy Metals . . . . . . . . . . . . . . . . . 48

3.1.2 Chemistry of Copper . . . . . . . . . . . . . . . 48

3.1.3 Copper Sorption Techniques . . . . . . . . . . . . 50

3.2 Results . . . . . . . . . . . . . . . . . . 58

3.2 .1 Sorption Studies $\ldots \ldots \ldots$. . . . . . . . . . . . . 58

3.2.2 Kinetics of Sorption . . . . . . . . . . . . . 67 
3.2.3 Surface Analysis . . . . . . . . . . . . . 76

3.2 .4 Mechanism of Sorption . . . . . . . . . . . . . . . . . 91

3.3 Conclusions . . . . . . . . . . . . . . . . . . 94

4 Magnetic Calcium Silicate Composites $\quad 96$

4.1 Introduction . . . . . . . . . . . . . . 96

4.1.1 Magnetism . . . . . . . . . . . . . . . . 96

4.1.2 Magnetic Separation ................ . 101

4.2 Results . . . . . . . . . . . . . . . . . 103

4.2.1 Superparamagnetic NCS Composites _. . . . . . . . . . . 103

4.2.2 Bulk Magnetite-NCS Composites . . . . . . . . . . . . 118

4.2 .3 Sorption Studies . . . . . . . . . . . . . 123

4.3 Conclusions . . . . . . . . . . . . . . . . . . . 128

4.3.1 Maghemite-Calcium Silicate Composites . . . . . . . . . . . 128

4.3.2 Magnetite-Calcium Silicate Composites . . . . . . . . . . . . . 130

5 Phosphate Sorption by $\mathrm{Fe}_{3} \mathrm{O}_{4}$-NCS Composites 131

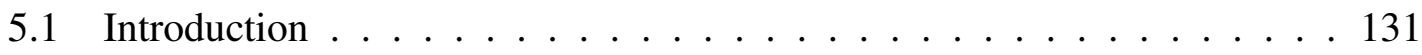

5.1 .1 Phosphorus Speciation . . . . . . . . . . . . . 131

5.1.2 Phosphate in the Environment . . . . . . . . . . . 131

5.1.3 Phosphate Sorption . . . . . . . . . . . . . 133

5.1.4 Biological Removal . . . . . . . . . . . . . . . . . . . 134

5.1.5 Chemical Removal . . . . . . . . . . . . . . . 136

5.1.6 Phosphate Sorption by NCS . . . . . . . . . . . . . 138

5.2 Results . . . . . . . . . . . . . . . . . . . . . 140

5.2 .1 Isotherm Studies . . . . . . . . . . . . . . . . . . . 140

5.2 .2 Kinetic Studies . . . . . . . . . . . . . . . . . . 147

5.2.3 Wastewater Treatment . . . . . . . . . . . . . . 149 
5.2.4 Continuous Uptake Studies . . . . . . . . . . . . . . . . . . . 149

5.3 Conclusions . . . . . . . . . . . . . . . . 156

6 Rhenium Sorption by Polyaniline-NCS Composite Materials 158

6.1 Introduction . . . . . . . . . . . . . . . 158

6.1 .1 Polyaniline ........................ 158

6.1.2 Chemisty of Rhenium . . . . . . . . . . . . 163

6.1.3 Rhenium Sorption . . . . . . . . . . . . . 165

6.2 Results . . . . . . . . . . . . . . . . . . . . 169

6.2.1 Polyaniline-NCS Composites . . . . . . . . . . . . 169

6.2.2 Thermogravimetric Analysis . . . . . . . . . . . 175

6.2 .3 Electrochemistry .................. 176

6.2.4 X-Ray Photoelectron Spectroscopy . . . . . . . . . . . . 179

6.2.5 Rhenium Sorption Isotherms . . . . . . . . . . . . . . . 190

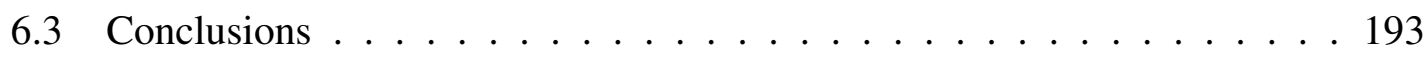

$7 \quad$ Summary of Thesis $\quad 196$

7.1 Sorption of Copper . . . . . . . . . . . . . . . 196

7.2 Magnetic Calcium Silicate Composites . . . . . . . . . . . . . . 197

7.3 Sorption of Phosphate by Magnetite-Calcium Silicate Composites . . . 198

7.4 Polyaniline Calcium Silicate Composites and Sorption of Rhenium . . . . 199

7.5 Future Work . . . . . . . . . . . . . . . . 200

A Data for Figures $\quad 202$

$\begin{array}{lr}\text { References } & 212\end{array}$ 


\section{List of Figures}

1.1 Scanning electron micrograph of nanostructured calcium silicate showing its porous microstructure . . . . . . . . . . . . . 16

1.2 Suggested formula and schematic of nanostructured calcium silicate. . . . 17

2.1 Schematic of set-up for column sorption experiments. . . . . . . . . . . . 44

3.1 Chitin and its deacetylated analogue, chitosan. . . . . . . . . . . 53

3.2 Co-polymer resin as used by Wang and Wang for copper sorption. . . . . 54

3.3 Hydroxide bridged copper dimer, as observed by Kurdoa et al. . . . . . . 55

3.4 Leaching of $\mathrm{Ca}^{2+}$ from NCS in contact with water. . . . . . . . 58

3.5 Sorption characteristics of NCS towards $\mathrm{Cu}^{2+}$ as either $\mathrm{CuCl}_{2}$ or $\mathrm{Cu}\left(\mathrm{NO}_{3}\right)_{2}$ an an initial concentration of approximately $80 \mathrm{mmol} \mathrm{dm}^{-3}$. . . . . 60

3.6 Sorption characteristics of NCS towards copper salts at lower concentrations. . . . . . . . . . . . . . . . . 62

3.7 Sorption characteristics of $\mathrm{pH}$ adjusted NCS towards copper salts. . . . . 64

3.8 Sorption characteristics of NCS towards $\mathrm{Cu}^{2+}$ in the presence of $\mathrm{I}_{3}^{-}$. . . 65

3.9 First- and second-order reaction models for sorption of $\mathrm{Cu}^{2+}$ by NCS. . . 69

3.10 Kinetics of hydrogen ion uptake by NCS in the presence of $1.6 \mathrm{mmol} \mathrm{dm}{ }^{-3}$

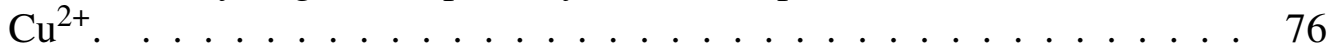

3.11 Powder X-ray diffraction patterns of spent NCS material following sorption at various initial concentrations of $\mathrm{Cu}\left(\mathrm{NO}_{3}\right)_{2} \ldots \ldots 77$

3.12 Powder X-ray diffraction patterns of spent NCS material following sorption of $\mathrm{Cu}^{2+}$ from $\approx 80 \mathrm{mmol} \mathrm{dm}^{-3}$ solution. . . . . . . . . . . 78

3.13 Electron micrographs of spent NCS material following sorption of $\mathrm{Cu}\left(\mathrm{NO}_{3}\right)_{2} .81$

3.14 Electron micrographs of spent NCS following sorption of $\mathrm{Cu}^{2+}$ from a $\mathrm{CuCl}_{2}$ solution. ................ 83

3.15 Electron micrographs of spent NCS material following sorption of $\mathrm{Cu}^{2+}$ from a $\mathrm{Cu}\left(\mathrm{NO}_{3}\right)_{2}$ solution at $1.6 \mathrm{mmol} \mathrm{dm}^{-3} \ldots \ldots . \ldots 85$

3.16 EDS overlay of NCS follwing sorption of $\mathrm{Cu}^{2+}$ from a $\mathrm{Cu}\left(\mathrm{NO}_{3}\right)_{2}$ solution. 87 
3.17 EDS overlay of $\mathrm{pH}$ adjusted NCS following sorption of $\mathrm{Cu}^{2+}$ from a $\mathrm{Cu}\left(\mathrm{NO}_{3}\right)_{2}$ solution. ..................... 88

3.18 EDS overlay of NCS following sorption of $\mathrm{Cu}^{2+}$ from a $\mathrm{CuCl}_{2}$ solution. . $\quad 90$

4.1 Formation of magnetic sublattices. . . . . . . . . . . . . . . . . 98

4.2 Formation of domains within a magnetic crystal. . . . . . . . . . . . 99

4.3 Magnetisation curve showing example of superparamagnetism. . . . . . . 100

4.4 Magnetic field gradient generated by the presence of a ferromagnetic wire in an otherwise uniform field. . . . . . . . . . . . . . . . . 102

4.5 Particle size distribution of $\mathrm{Fe}_{3} \mathrm{O}_{4}$-NCS slurries, prepared with superparamagnetic $\mathrm{Fe}_{3} \mathrm{O}_{4}$. . . . . . . . . . . . . . . . . . . . . 104

4.6 Surface area measurements on $\gamma-\mathrm{Fe}_{2} \mathrm{O}_{3}$-NCS composite materials. . . . . 106

4.7 X-ray diffraction pattern of superparamagnetic $\mathrm{Fe}_{3} \mathrm{O}_{4} \ldots \ldots$. . . . . 107

4.8 X-ray diffraction pattern for $\mathrm{Fe}_{3} \mathrm{O}_{4}$ - and $\gamma$ - $\mathrm{Fe}_{2} \mathrm{O}_{3}-\mathrm{NCS}$ composite materials. 108

4.9 XPS analysis of superparamagnetic $\gamma-\mathrm{Fe}_{2} \mathrm{O}_{3}-\mathrm{NCS}$ composite material. . . 110

4.10 Saturation magnetic moment for superparamagnetic $\gamma-\mathrm{Fe}_{2} \mathrm{O}_{3}-\mathrm{NCS}$ composites, prior and subsequent to copper sorption. . . . . . . . . . 111

4.11 Remnant magnetic moment for superparamagnetic $\gamma-\mathrm{Fe}_{2} \mathrm{O}_{3}-\mathrm{NCS}$ composites, prior and subsequent to copper sorption. . . . . . . . . . . . 112

4.12 EDS overlay of 2-ethoxyethanol washed $\gamma$-Fe ${ }_{2} \mathrm{O}_{3}$-NCS material. . . . . 114

4.13 Electron micrographs of $\gamma-\mathrm{Fe}_{2} \mathrm{O}_{3}$-NCS at increasing magnification. . . . 115

4.14 Magnetic separation of $\gamma-\mathrm{Fe}_{2} \mathrm{O}_{3}$-NCS composites. . . . . . . . . . 117

4.15 Particle size distribution of $\mathrm{Fe}_{3} \mathrm{O}_{4}$-NCS slurries prepared with bulk $\mathrm{Fe}_{3} \mathrm{O}_{4}$ powder. . . . . . . . . . . . . . . . 119

4.16 Effect of instrument absorbance setting on recorded particle size distribution of $\mathrm{Fe}_{3} \mathrm{O}_{4}$ powder. . . . . . . . . . . . . . . . . . . 120

4.17 X-ray diffraction pattern of $\mathrm{Fe}_{3} \mathrm{O}_{4}$-NCS composite material, prepared with bulk $\mathrm{Fe}_{3} \mathrm{O}_{4} \ldots \ldots \ldots \ldots 122$

4.18 Sorption characteristics of $\gamma-\mathrm{Fe}_{2} \mathrm{O}_{3}$-NCS towards $\mathrm{Cu}\left(\mathrm{NO}_{3}\right)_{2} \ldots \ldots$

4.19 Isothermal sorption of $\mathrm{Cu}^{2+}$ by $\mathrm{Fe}_{3} \mathrm{O}_{4}$-NCS composite. . . . . . . . . . 126

4.20 Electron micrograph of $\gamma-\mathrm{Fe}_{2} \mathrm{O}_{3}-\mathrm{NCS}$ following sorption of $\mathrm{Cu}^{2+}$. . . 127 
4.21 EDS overlay of $\gamma-\mathrm{Fe}_{2} \mathrm{O}_{3}-\mathrm{NCS}$ following sorption of $\mathrm{Cu}^{2+}$ from a $\mathrm{Cu}\left(\mathrm{NO}_{3}\right)_{2}$ solution. . . . . . . . . . . . . . . . . 129

5.1 Phosphate sorption capacity of $\mathrm{Fe}_{3} \mathrm{O}_{4}$-NCS composites. . . . . . . . . . 141

5.2 Phosphate sorption isotherms. . . . . . . . . . . . . . . 143

5.3 Sorption of $\mathrm{H}_{2} \mathrm{PO}_{4}^{-}$by $\mathrm{Fe}_{3} \mathrm{O}_{4}$-NCS composite. . . . . . . . . . . . . . . 148

5.4 Column-based continuous uptake of phosphate by $\mathrm{Fe}_{3} \mathrm{O}_{4}$-NCS. . . . . . 151

5.5 Experimental set-up for sorption of phosphate by $\mathrm{Fe}_{3} \mathrm{O}_{4}$-NCS. . . . . . 155

6.1 Redox states of polyaniline. . . . . . . . . . . . . . . . . . . 160

6.2 Mechanism for the oxidation of aniline. . . . . . . . . . . . . . 161

6.3 Poly(2-methoxyaniline-5-sulfonic acid) (PMAS) in the leucoemeraldine base state. . . . . . . . . . . . . . . . . . 162

6.4 Electrochemical potential for the reaction of polyaniline and $\mathrm{ReO}_{4}^{-}$. . . . 164

6.5 Examples of rhenium(VII) complexes exhibiting Re-N bonding. . . . . . 165

6.6 Polymer resin used by Xiong et al. for $\mathrm{ReO}_{4}^{-}$sorption. . . . . . . . . . . 166

6.7 Examples of hydrogen bonding between $\mathrm{ReO}_{4}^{-}$and amine species. . . . . 168

6.8 Amine functional groups reported as suitable for ion exchange of $\mathrm{ReO}_{4}^{-}$ by Kholmogorov et al. . . . . . . . . . . . . . . . . . . . . . . 168

6.9 UV/vis spectra of polyaniline, and PANI-NCS composite materials. . . . 170

6.10 Reflectance spectra of NCS and its PANI composite. . . . . . . . . . . . 170

6.11 Possible hydrogen bonding modes between emeraldine base and NCS. . . 171

$6.12 \mathrm{UV} / \mathrm{vis}$ spectra of perrhenate sorption onto polyaniline films. . . . . . . . 173

6.13 UV/vis spectra of polyaniline films deposited on polycarbonate cuvettes. . 174

6.14 TGA trace for PANI-NCS composite with a nominal $10 \mathrm{wt} \%$ PANI content.175

6.15 Cyclic voltagram of PANI-NCS composite. . . . . . . . . . . . 176

6.16 Pourbaix diagram for the rhenium system. . . . . . . . . . . . . 177

6.17 X-ray photoelectron spectra of polyaniline as emeraldine salt: (a) ${ }^{14} \mathrm{~N}$ PANI; (b) ${ }^{15}$ N-PANI. . . . . . . . . . . . . . . . . . . . 180 
6.18 X-ray photoelectron spectra of polyaniline-NCS composite material prior to rhenium sorption. . . . . . . . . . . . . . . . 183

6.19 X-ray photoelectron spectra of unmodified 2-ethoxyethanol washed NCS. 184

6.20 X-ray photoelectron spectra of polyaniline-NCS composite material following rhenium sorption. . . . . . . . . . . . . . 186

6.21 X-ray photoelectron spectra of $\mathrm{KReO}_{4} \ldots \ldots \ldots$. . . . . . . . . 187

6.22 Isotherm models for the sorption of $\mathrm{ReO}_{4}^{-}$by PANI-NCS composite under acidic conditions. . . . . . . . . . . . . . . . . 191

6.23 Supernatant $\left[\mathrm{Ca}^{2+}\right]$ and $\mathrm{pH}$ for the $\mathrm{ReO}_{4}^{-}$sorption isotherm presented in Figure 6.22 . . . . . . . . . . . . . . . . . . . 192 


\section{List of Tables}

1.1 Excerpt from Drinking-water Standards for New Zealand. . . . . . . . . . 3

1.2 Excerpt from Wellington City Trade Waste Bylaw. . . . . . . . . . . . . . 4

1.3 Effects of inverse or logarithmic transformations on uncertainties. . . . . 10

1.4 Typical elemental composition of NCS as determined by XRF. . . . . . . 17

1.5 Surface tension of various solvents. . . . . . . . . . . . . . . . . . 19

2.1 Instrumental conditions for atomic absorption analysis. . . . . . . . . . 29

3.1 Solubility products and stability constants of some metal-containing species. 49

3.2 Rate data for the sorption of $\mathrm{Cu}^{2+}$ from a $\mathrm{Cu}\left(\mathrm{NO}_{3}\right)_{2}$ solution by NCS. . . 70

3.3 Rate data for $\left[\mathrm{OH}^{-}\right]$in sorption of copper by NCS. . . . . . . . . . . 75

4.1 Definition of some quantities associated with magnetism. . . . . . . . . 96

4.2 Parameters for X-ray diffraction pattern shown in Figure 4.7. . . . . . . 108

4.3 Parameters for the XPS peaks modelled in Figure 4.9 . . . . . . . . . . . 109

4.4 Effect of material absorbance on recorded particle size distribution of $\mathrm{Fe}_{3} \mathrm{O}_{4}$ powder. . . . . . . . . . . . . 120

4.5 Parameters for X-ray diffraction pattern shown in Figure 4.17. . . . . . 121

4.6 Parameters for copper sorption isotherms as shown in Figure 4.19. . . . . 125

5.1 Phosphate equilibria in aqueous conditions. . . . . . . . . . . . . 131

5.2 Common metal species encountered as counter-ions in phosphate precipitates. . . . . . . . . . . . . . . . . . . 132

5.3 Calcium compounds of phosphorus commonly encountered in soils. . . . 133

5.4 Parameters for isotherm models of phosphate sorption by $\mathrm{Fe}_{3} \mathrm{O}_{4}$-NCS composite material. . . . . . . . . . . . . . . . 146

5.5 Rate data for phosphate sorption by $\mathrm{Fe}_{3} \mathrm{O}_{4}$-NCS composite. . . . . . . . . 147

5.6 Column-based continuous uptake of phosphate by $\mathrm{Fe}_{3} \mathrm{O}_{4}$-NCS. . . . . . 152

6.1 Reduction potentials of various group 7 species. . . . . . . . . . . . . 163 
6.2 Equilibria determining rhenium uptake by chitosan. . . . . . . . . . . 167

6.3 Spectral parameters for PANI-NCS composite, from spectra presented in Figure 6.9. . . . . . . . . . . . . . . . . . . 171

6.4 Parameters for X-ray photoelectron spectra of polyaniline samples. . . . . 179

6.5 Parameters for X-ray photoelectron spectra of PANI-NCS composite materials. . . . . . . . . . . . . . . . . . 182

6.6 Parameters for X-ray photoelectron spectra of PANI-NCS composite following rhenium sorption from $\mathrm{K}^{\mathrm{KReO}_{4}}$ solution. . . . . . . . . 188

6.7 Parameters for perrhenate sorption isotherms as shown in Figure 6.22. . . 191 


\section{Publications}

The following paper has been published as a result of the work undertaken in Chapter 3, Copper Sorption:

Cairns, M. J.; Borrmann, T.; Höll, W. H.; Johnston, J. H. A study of the uptake of copper ions by nanostructured calcium silicate Microporous and Mesoporous Materials 2006, 95, 126-134. 


\section{Glossary}

AA (Flame) atomic absorption (spectroscopy)

ADP Adenosine diphosphate

ADP Adenosine triphosphate

ANS 1-Amino-2-naphthol-4-sulfonic acid

BET (isotherm) The Brunauer, Emmett and Teller isotherm

DRP Dissolved reactive (toward the molybdate test) phosphorus

EB Emeraldine base of polyaniline

EDS Energy dispersive spectroscopy

EDTA Ethylenediaminetetraacetic acid/ acetate

EPR Electron paramagnetic resonance (spectroscopy)

ES Emeraldine salt of polyaniline

FZK Forschungszentrum Karlsruhe (Research centre Karlsruhe, Karlsruhe, Germany)

GV Guideline value, c.f. MAV

HGMS High gradient magnetic separation

HWHM Half peak width at half median height

ICDD International Centre for Diffraction Data

LD $\quad$ Median lethal dose

LEB Leucoemeraldine base of polyaniline

MAS Monomer of PMAS, 3-amino-4-methoxybenzenesulfonic acid

MAS-NMR Magic angle spinning nuclear magnetic resonance (spectroscopy)

MAV Maximum acceptable value

NCS Nanostructured calcium silicate

NDF Number of degrees of freedom

NMP N-methyl pyrrolidine 
PANI Polyaniline

PDF Powder diffraction file

PMAS Poly(2-methoxyaniline-5-sulfonic acid)

PNB Pernigraniline base of polyaniline

PNS Pernigraniline salt of polyaniline

$R^{2} \quad$ Coefficient of determination

RED Reducing agent (for phosphate analysis)

SEM Scanning electron microscopy

SMOR Molybdic acid reagent (for phosphate analysis)

TDP Total dissolved phosphorus

TGA Thermogravimetric analysis

TP Total phosphorus

TRP Total reactive phosphorus

UV/vis Ultra Violet/visible absorption (spectroscopy)

wt \% $\quad$ Percent by mass

XPS X-ray photoelectron spectroscopy

XRD (Powder) X-ray diffraction

XRF X-ray fluorescence (spectroscopy) 


\section{Chapter 1}

\section{Introduction}

\subsection{Water Pollution and the Environment}

In 2006 in New Zealand, it has been estimated that resource consents permit the withdrawl of water from limnological sources at a rate of up to $676 \mathrm{~m}^{3} \mathrm{~s}^{-1}$ for anthropogenic purposes other than hydroelectric power generation. ${ }^{1}$ The actual rate at which this water is used is often much less than this, and varies according to temporal climatic conditions. A large portion of this water becomes contaminated through use, and has the potential to cause significant environmental damage if discharged without adequate treatment. Typical contamination includes:

- Biological contaminants, especially pathogens often found in fecal matter from agricultural wastes or sewage.

- Organic waste such as sugars, proteins, lipids, aromatic hydrocarbons, pesticides etc. from a variety of agricultural and food processing sources.

- Nutrients such as phosphate and nitrate from agricultural waste.

- Other inorganic species with adverse effects on human health such as copper, lead, cadmium and fluoride from industrial processes.

Other metal ions, particularly radionucleotides such as caesium, strontium and technetium are not a significant source of waste-water contamination in New Zealand. However, countries utilising nuclear power generation must deal with significant volumes of such waste. While the aforementioned contaminants can have significant adverse effects on the environment, many are potentially valuable and deserve recycling. An efficient, cost effective recovery system would allow the materials to be recycled thus reducing both pollution and depletion of mineral resources. 
From the New Zealand perspective, agricultural wastewaters are a major concern. Agricultural run-off typically contains high concentrations of nitrogen species (such as ammonium) and phosphate. Smith, ${ }^{2}$ in a 20 month study from 1984-1985 of water runoff in a pastoral area near Hamilton, New Zealand, found total phosphorus concentrations averaging $1 \mathrm{mg} \mathrm{dm}^{-3}$ (as $\mathrm{P}$ ) and total nitrogen concentrations averaging $6 \mathrm{mg} \mathrm{dm}^{-3}$ (as $\mathrm{N})$. The rate of biological activity in aquatic systems is often limited by the concentration of these nutrients, especially phosphate. ${ }^{3}$ When excess phosphate enters the aquatic environment through such run-off, the rate of biological activity drastically increases, particularly for organisms such as algae. ${ }^{4}$ This can lead to eutrophication of the waterway: bacterial decomposition of the algae significantly reduces the dissolved oxygen content in the water, leading to death of higher organisms such as fish. If these nutrients can be recovered before they enter riparian zones and associated waterways, they may be reapplied to agricultural areas as fertiliser.

\subsubsection{Water Quality}

In New Zealand, the Resource Management Act 1991 provides the basis for legislation designed to regulate pollution and to mitigate its environmental impact. ${ }^{5}$ In conjunction with this act, government departments and local government bodies construct regulations specifying maximum acceptable values (MAVs) for various constituents of wastewater such that effluent from any treatment process applied to the wastewater poses negligible risk to either the environment or to human health. Ideally, after adequate mixing of the effluent and the water to which it is discharged, there should be no noticeable change in the local environmental conditions. Example of such regulations are the Drinking-water Standards for New Zealand ${ }^{6}$ and the Wellington City Council Trade Waste Bylaw 2004, ${ }^{7}$ excerpts of which are shown below.

\subsubsection{Potable Water}

The Ministry of Health promulgates regulations specifying the criteria that water supplies must meet before those supplies may be considered potable. These regulations specify, 
among other things, the maximum allowable concentration of analytes whose characteristics are potentially detrimental to human health. The MAVs of some of the regulated inorganic species are shown in Table 1.1. This list is not exhaustive; other analytes, including organic species, bacteria and protozoa are also regulated. While potable water is by no means chemically pure, these standards provide a suitable guide for water quality.

Table 1.1 Excerpt from Drinking-water Standards for New Zealand. ${ }^{6}$

\begin{tabular}{|c|c|c|c|}
\hline Analyte & $\begin{array}{l}\text { MAV/ }{ }^{a} \\
\mathbf{m g ~ d m}^{-3}\end{array}$ & $\begin{array}{l}\mathbf{G V} /^{b} \\
\mathbf{m g ~ d m ^ { - 3 }}\end{array}$ & Notes \\
\hline Arsenic & 0.01 & - & \\
\hline Cadmium & 0.004 & - & \\
\hline Chromium & 0.05 & - & $\begin{array}{l}\text { Includes all valent } \\
\text { forms }\end{array}$ \\
\hline Copper & 2 & 1 & $\begin{array}{l}\text { Set to prevent stain- } \\
\text { ing. }\end{array}$ \\
\hline Fluoride & 1.5 & $0.7-1.0$ & $\begin{array}{l}\text { Guideline set for oral } \\
\text { health reasons }\end{array}$ \\
\hline Lead & 0.01 & - & \\
\hline Mercury & 0.002 & - & \\
\hline Nickel & 0.02 & - & \\
\hline Nitrate & 50 & - & Measured as $\mathrm{NO}_{3}$ \\
\hline Nitrite $^{c}$ & 0.2 & - & Measured as $\mathrm{NO}_{2}$ \\
\hline $\begin{array}{l}\text { Phosphate } \\
\text { Hardness }\end{array}$ & - & - & Not regulated \\
\hline $\begin{array}{l}(\mathrm{Ca} \text { and } \\
\mathrm{Mg})\end{array}$ & - & $100-300$ & Measured as $\mathrm{CaCO}_{3}$ \\
\hline Dissolved solids & - & 1000 & \\
\hline $\mathrm{pH}$ & - & $7.0-8.5$ & \\
\hline
\end{tabular}

${ }^{a}$ Maximum Acceptable Values are specified such that a person consuming 2 litres per day over a lifetime of 70 years will experience no statistically significant risk of adverse health effects as a result.

${ }^{b}$ Guideline Values are set for aesthetic purposes.

${ }^{c}$ The value for nitrate is set as a short-term limit. No long-term limit is given for nitrate; however, the value listed for nitrite is a long-term exposure limit.

\subsubsection{Trade Wastes}

In Wellington, the composition of wastewater resulting from industrial processes must meet certain criteria before it may be discharged into the municipal sewer. Table 1.2 gives criteria for some of the inorganic constituents that may be found in such wastes. In general, any discharges must not jeopardise the correct functioning of the municipal wastewater treatment system. The other consideration undertaken in setting these limits 
is, whether the analyte can be successfully removed from the treated effluent that is discharged. The municipal wastewater treatment facility in Wellington consists of five processes: screening, primary sedimentation, microbiological oxidation, secondary sedimentation, and UV disinfection. The treated effluent is subsequently discharged into the sea. ${ }^{8}$ Based on these processes, the trade waste limits in Table 1.2 can be understood in the following way:

- Limits for most of the metal species, such as arsenic or copper are at moderate levels, as they are precipitated (for example as copper hydroxides or iron arsenates) in the sedimentation processes.

- The limit for mercury is set at a low level, ${ }^{\dagger}$ because if mercury is inadequately removed, it will bio-accumulate in the aquatic environment.

- Limits for nutrients, such as nitrogen- and phosphorus-containing species are set at high levels, similar to those encountered in municipal sewage. The microorganisms involved in the biological oxidation process consume these species.

Table 1.2 Excerpt from Wellington City Trade Waste Bylaw. ${ }^{7}$

\begin{tabular}{lr}
\hline Analyte & MAV/ $\mathbf{~ g ~ d m ~}^{-\mathbf{3}}$ \\
\hline Arsenic & 5 \\
Cadmium & 0.5 \\
Copper & 10 \\
Fluoride & 30 \\
Lead & 10 \\
Mercury & 0.005 \\
Nickel & 10 \\
Nitrogen & $a$ \\
pH & 500 \\
Phosphorus & 10.0 \\
\hline
\end{tabular}

\footnotetext{
${ }^{a}$ This limit is for nitrogenous species that are converted to ammonia by the Kjeldahl distillation procedure. ${ }^{10}$ This includes ammonia, ammonium, amines and amides, but not nitrate, nitrite or other nitrogen species nonreactive towards concentrated $\mathrm{H}_{2} \mathrm{SO}_{4}$. The value is reported as $\mathrm{mg} \mathrm{N} \mathrm{dm}^{-3}$.
}

This thesis investigates the sorption of ionic contaminants from aqueous solution. As such, it is advisable to discuss sorption models.

\footnotetext{
${ }^{\dagger}$ Under optimum conditions, instrumental detection limits can be significantly lower than the MAV. For example, Chou and Naleway, have reported a detection limit for the analysis of mercury by cold vapour atomic absorption spectroscopy at $0.013 \mu \mathrm{g} \mathrm{dm}^{-3} .^{9}$
} 


\subsection{Sorption Models}

\subsubsection{Sorption Isotherms}

A sorption isotherm represents, in its simplest form, the state of the system at equilibrium. When a discussion of sorption isotherms is undertaken, it is convenient to define $\theta$, the fraction of surface coverage, as the ratio of sorption sites occupied by a sorbent, $n$, to the total number of sites present in the system, $n_{\infty}$ :

$$
\theta=\frac{n}{n_{\infty}}
$$

\subsubsection{The Langmuir Isotherm}

Of the isotherms commonly used to describe sorption systems, the Langmuir isotherm is one of the few that may be derived from fundamental principals. The isotherm was originally developed to describe the sorption of gases onto the surface of either glass, mica or platinum. ${ }^{11}$ In his derivation of the functional form of the isotherm, Langmuir approached the problem from a consideration of the relative rates of sorption and desorption. A similar approach is given below.

The equilibrium between a sorbate, $\mathrm{M}$, bound to a sorbent site, A, can be defined as follows:

$$
\mathrm{A}(\mathrm{s})+\mathrm{M}(\mathrm{aq}) \underset{k_{-1}}{\stackrel{k_{1}}{\rightleftharpoons}} \mathrm{A}-\mathrm{M}(\mathrm{s})
$$

From this, it is possible to construct the rate law with respect to the number of occupied sites, $n_{\mathrm{A}-\mathrm{M}}$ :

$$
\frac{d n_{\mathrm{A}-\mathrm{M}}}{d t}=k_{1} \cdot n_{\mathrm{A}} \cdot[\mathrm{M}]-k_{-1} \cdot n_{\mathrm{A}-\mathrm{M}}
$$


then $\theta$ can be defined in terms of the actual species present:

$$
\theta=\frac{n_{\mathrm{A}-\mathrm{M}}}{n_{\mathrm{A}}+n_{\mathrm{A}-\mathrm{M}}}
$$

or, in terms of occupied sites:

$$
n_{\mathrm{A}-\mathrm{M}}=\frac{n_{\mathrm{A}} \cdot \theta}{1-\theta}
$$

When the system reaches equilibrium, the net rate of reaction becomes zero. It becomes possible to combine (1.5) and (1.3), eliminating both $n_{\mathrm{A}}$ and $n_{\mathrm{A}-\mathrm{M}}$ :

$$
\frac{k_{1} \cdot[\mathrm{M}]_{E q}}{k_{-1}}=\frac{\theta}{1-\theta}
$$

Given that the ratio of forward to reverse rates is equivalent to the overall equilibrium constant of the reaction, $K=\frac{k_{1}}{k_{-1}}$, Equation (1.6) is functionally identical to the Langmuir isotherm:

$$
\theta=\frac{K[\mathrm{M}]_{E q}}{1+K[\mathrm{M}]_{E q}}
$$

As the coefficient for this isotherm is approximately equal to the equilibrium constant, ${ }^{\dagger}$ it is possible to derive other thermodynamic values once the system has been shown to conform to the Langmuir isotherm.

One of the drawbacks to this approach is that it assumes that all sorption sites within the system are uniform - that is to say that sorption does not occur preferentially at an sorption site (or group of sites) within the system. Another assumption made in the derivation of this isotherm is that interactions do not occur between different sorbed molecules. Such interactions may occur, for example, in cases where the presence of one sorbed molecule promotes (or retards) the rate at which sorption occurs at adjacent sites.

\footnotetext{
${ }^{\dagger}$ It is recognised that the equilibrium constant for (and, by analogy, reaction rates of) a system are not derived directly from the concentration of the species involved, but from their activities. If, in the example shown above, $\mathbb{K}$, the equilibrium constant, is defined as $\mathbb{K}=\frac{a_{\mathrm{A}-\mathrm{M}}}{a_{\mathrm{A}} a_{\mathrm{M}}}$ then $K=\frac{\gamma_{\mathrm{A}} \gamma_{\mathrm{M}}}{\gamma_{\mathrm{A}-\mathrm{M}}} \mathbb{K}$ where $a_{x}$, the activity of component $x$, is defined as $a_{x}=\gamma_{x}[x]$. In systems for which species are present at sub-molar concentrations, the values of $\mathbb{K}$ and $K$ are often similar.

As this study concerns the sorption of species at concentrations of a few millimolar from aqueous solution, it is appropriate to approximate $\mathbb{K}$ with $K$ in the present work.
} 
Interaction between sorbed species can also lead to the formation of multiple sorption layers (as opposed to the formation of monolayers of sorbed species). The presence of these interactions leads to different rates of sorption at different sites within the system. While the system can still be described by the Langmuir isotherm, its treatment becomes more complex. An example of this follows. In cases where different types of sorption sites are known to exist within the system, $\mathrm{A}_{1}$ to $\mathrm{A}_{n}$, the rate at which sorption occurs will differ depending on the type of site. If the fraction of site $i$ is represented by $\beta_{i}$, then the Langmuir isotherm becomes:

$$
\theta=\sum_{i=1}^{n} \frac{\beta_{i} K_{i}[\mathrm{M}]_{E q}}{1+K_{i}[\mathrm{M}]_{E q}} \quad \sum_{i=1}^{n} \beta_{i}=1
$$

The introduction of multiple variables makes the fitting of such a model to a set of experimental data more difficult. As such, this is rarely done.

Another example for which the Langmuir model is insufficient is the presence of multiple layers of sorbate forming on the surface of the sorbent. For example, should the reaction presented in Equation (1.2) be:

$$
\mathrm{A}(\mathrm{s})+\mathrm{M}(\mathrm{aq}) \underset{k_{-1}}{\stackrel{k_{1}}{\rightleftharpoons}} \mathrm{A}-\mathrm{M}(\mathrm{s}) \stackrel{+\mathrm{M}(\mathrm{aq}) \quad k_{2}}{\underset{k_{-2}}{\rightleftharpoons}} \mathrm{A}-\mathrm{M}-\mathrm{M}(\mathrm{s}) \rightleftharpoons \ldots
$$

The rate law presented in (1.3) must be extended to account for the formation of additional sorbate layers. Once this is done, expressing the surface coverage in terms of the equilibrium concentration of $\mathrm{M}$ becomes more complex, and other isotherm models (given below) become more appropriate.

\subsubsection{The Freundlich and Tempkin Isotherms}

The Freundlich and Tempkin isotherms were developed in an attempt to account for the non-conformity to the Langmuir isotherm by systems exhibiting multi-layer sorption, or preferential sorption sites. The adaptation of the Langmuir isotherm results in isotherm parameters exhibiting complex relationships to, for example, the system equilibrium 
constant, K. As such, while these isotherm models may, through empirical techniques, be shown to adequately describe a system, applying the information acquired from one system, to another is a difficult task. The Freundlich isotherm is given by ${ }^{12}$

$$
\theta=a\left([\mathrm{M}]_{E q}\right)^{\frac{1}{C_{2}}}
$$

While the Tempkin isotherm is given by ${ }^{13}$

$$
\theta=C_{1} \ln \left(C_{2}[\mathrm{M}]_{E q}\right)
$$

In the equations (1.10) and (1.11) above, $a, C_{1}$ and $C_{2}$ are parameters dependent on the individual system, whereas $[\mathrm{M}]_{E q}$ is the concentration of the sorbate present in solution at equilibrium.

\subsubsection{The Frumkin Isotherm}

The Frumpkin isotherm is derived from the Langmuir isotherm by introducing a term to describe inhomogeneity of the sorbent surface. If the Langmuir isotherm, Equation (1.7), is expressed as follows: ${ }^{12,14}$

$$
K[\mathrm{M}]_{E q}=\frac{n}{n_{\infty}-n}
$$

Then the Frumkin isotherm is arrived at through the addition of an inhomogeneity term, $A$ :

$$
K[\mathrm{M}]_{E q}=\frac{n}{n_{\infty}-n} \exp \left(-A \frac{n}{n_{\infty}}\right)
$$

Or, in terms of $\theta$ :

$$
\theta=\frac{K[\mathbf{M}]_{E q}}{K[\mathbf{M}]_{E q}+\exp (-A \theta)}
$$

Unfortunately, in the case of the Frumkin isotherm, $\theta$ cannot be expressed as a function of $[\mathrm{M}]_{E q}$ as it can be in the other isotherms discussed previously, making a direct comparison of isotherms (by non-graphical means) difficult. 


\subsubsection{The BET Isotherm}

The BET isotherm ${ }^{\dagger}$ was derived from the Langmuir isotherm by allowing for the formation of multiple sorption layers on a surface (as is shown in the equilibrium presented in Equation (1.9)). ${ }^{15}$ This commonly occurs with the sorption of gasses at temperatures slightly above their boiling point. The isotherm model, as applicable to the sorption of gasses is:

$$
\begin{aligned}
\frac{V}{V_{m o n}} & =\frac{c z}{(1-z)[1-(1-c) z]} \\
z & =\frac{p}{p^{*}} \\
c & \approx \mathrm{e}^{\left(\frac{-\Delta G}{R T}\right)}
\end{aligned}
$$

where $V$ is the volume of gas sorbed, $V_{m o n}$ is the volume occupied by a sorbed monolayer, $p$ is the pressure in the system, $p^{*}$ is the saturation pressure of the gas at the temperature of interest and $c$ is a constant. The isotherm may be adapted to solution-based sorption by redefining $z$ as the ratio of $[\mathrm{M}]$ to the solubility of $\mathrm{M},[\mathrm{M}]^{*}$ :

$$
\begin{aligned}
\frac{n}{n_{\text {mon }}} & =\frac{c z}{(1-z)[1-(1-c) z]} \\
z & =\frac{[\mathrm{M}]}{[\mathrm{M}]^{*}}
\end{aligned}
$$

\subsubsection{Direct Fitting Models}

Most of the above isotherm models have linear implementations, approximating the function $f(x)=m x+c$, allowing the models to be applied to experimental data in a fairly straight-forward fashion. For example, the linear implementation of the Langmuir isotherm, Equation (1.7), is:

$$
\frac{1}{\theta}=\frac{1}{K} \frac{1}{[\mathbf{M}]_{E q}}+1
$$

${ }^{\dagger} \overline{\text { Named after Brunauer, Emmett and Teller }}$ 
while the linear form of the Freundlich isotherm, Equation (1.10), is:

$$
\log \theta=\frac{1}{C_{2}} \log [\mathrm{M}]_{E q}+\log a
$$

Thus if a system conforms to the Langmuir isotherm, a plot of $\frac{1}{\theta}$ against $\frac{1}{[\mathrm{M}]_{E q}}$ will exhibit a linear relationship. Likewise, for the Freundlich isotherm, a linear relationship will be observed in a plot of $\log \theta$ against $\log [\mathrm{M}]_{E q}$. While useful for checking if an individual model conforms to experimental data, difficulty is encountered if this system is used to decide which isotherm best models a specific data set, as comparing data normally, inversely or logarithmically has different effects on uncertainties associated with the data points. For example, in Table 1.3, the two data values each have a 5\% uncertainty. Normally, the absolute magnitude of this uncertainty increases with an increase in the data value; for the inverse, the absolute magnitude of this uncertainty decreases with an increase of the data value; for the logarithmic scale, the absolute magnitude of the uncertainty is independent of the original data value. This has consequences, if attempts are made to compare 'goodness of fit' criteria like $R^{2}$ values between different models, as any deviation from the ideal fit would be accorded different significance, depending on the space under which the model was fitted.

Table 1.3 Effects of inverse or logarithmic transformations on uncertainties.

\begin{tabular}{clll}
\hline Datum & \multicolumn{3}{c}{ Spread } \\
\cline { 2 - 4 }$(x \pm y)$ & Normal $(2 y)$ & Inverse $\left(\frac{1}{x-y}-\frac{1}{x+y}\right)$ & Logarithm $\left(\log \frac{x+y}{x-y}\right)$ \\
\hline $10.0 \pm 0.5$ & 1.0 & 0.010 & 0.043 \\
$100 \pm 5$ & 10. & 0.0010 & 0.043 \\
\hline
\end{tabular}

For this reason, when different isotherm models are fitted to the same data set in this study, parameters such as $R^{2}$ or $\chi^{2}$ values are calculated directly from the data values, not their inverse or logarithmic versions. Therefore, in this thesis, curve fitting software employing the Levenberg-Marquardt algorithm ${ }^{16}$ was used for the purposes of modelling the data. As such, linear interpretations of the isotherm models were not evaluated.

Choy et al. ${ }^{17}$ have observed the same discrepancy between $R^{2}$ values when comparing isotherm models for sorption of dyes onto activated carbon. 


\subsubsection{Diffusion-Controlled Sorption}

In sorbate-sorbent systems, the distribution of species within the system is not homogeneous. Typically the sorbent is a solid material and the sorbate is some species dissolved in solution. This means that the sorption process occurs at the phase boundary between the sorbent and the solution. ${ }^{\dagger}$ As such, there exists a gradient in the concentration of the sorbate as one moves from the bulk solution to the sorbent surface. If the sorbent particles have a large volume with respect to their surface area, a second diffusion process, wherein the sorbed species migrate from the surface of the particle into its centre, may also occur. Therefore, it is prudent to include a discussion of diffusion, when one considers such systems.

The fundamental concepts of diffusion are covered in the two laws named for Fick. ${ }^{13}$ Fick's first law states that under steady-state conditions, the flux of a particular component, $J_{\mathrm{C}}$, is proportional to the concentration gradient of that component:

$$
J_{\mathrm{C}}=-D \frac{\partial[\mathrm{C}]}{\partial x}
$$

If the system is under dynamic conditions, as opposed to the steady state, then the rate of change in the concentration of a particular component may be described as:

$$
\frac{\partial[\mathrm{C}]}{\partial t}=D \frac{\partial^{2}[\mathrm{C}]}{\partial x^{2}}
$$

Equations (1.22) and (1.23) are known as Fick's first and second laws.

Boyd, Adamson and Myers ${ }^{18}$ presented solutions for the kinetics of sorption where the rate limiting step was one of the aforementioned types of diffusion. Their work is discussed in more detail below.

\footnotetext{
For the purposes of this discussion this would typically be the phase boundary occurring between a solid and an aqueous ionic solution. The same concepts apply to other biphasic systems such as solid-gas or gas-liquid.
} 


\subsubsection{Diffusion Through a Liquid Film}

Should the kinetics of sorption be rate limited by diffusion of the sorbate species through the solution at the particle interface, the rate law takes the following form:

$$
\frac{d Q}{d t}=R\left(Q_{E q}-Q\right)
$$

which, when integrated with respect to time, becomes:

$$
Q=Q_{E q}\left(1-\mathrm{e}^{(-R t)}\right)
$$

where $Q$ and $Q_{E q}$ are the amount of substance sorbed by the sorbent at time $t$, and at equilibrium, $t=\infty$, and $R$ is defined as follows:

$$
R=\frac{3 D}{r . \Delta r \kappa}
$$

where $D$ is the diffusion coefficient, $r$ is the radius of the sorbent particle, $\Delta r$ is the thickness of the diffusion layer and $\kappa$ is the the distribution coefficient (defined as the ratio of the equilibrium sorbate concentration in the solid to the concentration in the solution). This relationship assumes that $D, \Delta r$ and $\kappa$ are constant throughout the approach towards equilibrium. This only holds true in cases where the sorbate is at infinite dilution. However the model can be approximated in cases where the sorbate is present at low concentrations. This often necessitates the use of radioactive tracer techniques to accurately determine the low concentrations of the sorbate.

This form of the rate law is identical to that exhibited by simple first order kinetics.

\subsubsection{Diffusion Through the Sorbent Particle}

In situations where the rate limiting step is the process of diffusion of the sorbate from the surface of the sorbent into its centre, the form of the equation is slightly different. The 
integrated form of the rate law is:

$$
\frac{Q}{Q_{E q}}=\sum_{n=1}^{\infty} \frac{1}{n^{2}} \exp \frac{-D n^{2} \pi^{2} t}{r^{2}}
$$

For cases where the system is significantly distant from equilibrium $\left(\frac{Q}{Q_{E q}}<0.05\right)$, the above equation simplifies to:

$$
\begin{aligned}
& \frac{Q}{Q_{E q}}=\frac{6}{\sqrt{\pi^{3}}} \sqrt{B t} \\
& \frac{Q}{Q_{E q}} \approx 1.08 \sqrt{B t}
\end{aligned}
$$

where

$$
B=\frac{D \pi^{2}}{r^{2}}
$$

As indicated above, the relationship in Equation (1.28) only holds true in systems for which the attainment of equilibrium is slow. Only in those cases can this method be employed. Otherwise, a special experimental set-up is required to facilitate the measurement of sorbate concentrations after short periods of sorbent-sorbate contact. This may, for example, be possible in systems, where the sorbate and the sorbent-sorbate complex exhibit significantly different spectral characteristics, enabling spectrophotometric techniques to be employed for analysis.

Even though Equation (1.27) holds true throughout the approach to equilibrium, using this equation to model an experimental data set is often too complex unless the assumptions for (1.28) can be applied.

\subsubsection{Kinetics of Sorption}

In the previous section, it was noted that most sorption systems are controlled by diffusion. Except in cases of infinite dilution, and in systems far removed from equilibrium, the diffusion limited steps may not be apparent in the overall rate law, and the system may exhibit behaviour more akin to typical solution based kinetics. That is to say that sorption 
systems often exhibit simple first- or second-order kinetics.

In a review of many studies of the sorption of divalent metal species, for example $\mathrm{Cd}(\mathrm{II})$, $\mathrm{Cu}(\mathrm{II})$ and $\mathrm{Pb}(\mathrm{II})$, Ho and $\mathrm{McKay}{ }^{19}$ reported that most of the experimental data exhibited a high degree of conformation to pseudo second-order kinetic models. The use of a second order model can be justified as follows. If the rate limiting step in the sorption process is not diffusion, but rather the binary reaction between the sorbent and sorbate, the reaction and its associated rate law are the same as for the derivation of the Langmuir isotherm, given in Equations (1.2) and (1.3). Assuming that the system is distant from equilibrium, so that the rate of the reverse reaction is negligible, and expressing all quantities in terms of amount of substance, Equation (1.3) becomes:

$$
\frac{d n_{\mathrm{A}-\mathrm{M}}}{d t}=k_{1} n_{\mathrm{M}} n_{\mathrm{A}}
$$

By mass balance,

$$
n_{\mathrm{A}}=n_{\mathrm{A}}^{0}-n_{\mathrm{M}}^{0}+n_{\mathrm{M}}
$$

where $n_{\mathrm{X}}^{0}$ is the initial amount of substance $\mathrm{X}$, then the rate can be expressed solely in terms of $n_{\mathrm{M}}$ :

$$
\begin{aligned}
\frac{d n_{\mathrm{A}-\mathrm{M}}}{d t} & =k_{1} n_{\mathrm{M}}\left(n_{\mathrm{A}}^{0}-n_{\mathrm{M}}^{0}+n_{\mathrm{M}}\right) \\
& =k_{1}\left[n_{\mathrm{M}}\left(n_{\mathrm{A}}^{0}-n_{\mathrm{M}}^{0}\right)+n_{\mathrm{M}}^{2}\right]
\end{aligned}
$$

In cases where the initial amounts of $\mathrm{A}$ or $\mathrm{M}$ are similar $\left(n_{\mathrm{A}}^{0} \approx n_{\mathrm{M}}^{0}\right)$, the rate law simplifies to the classic form for a second order process:

$$
\frac{d n_{\mathrm{A}-\mathrm{M}}}{d t}=k_{1} n_{\mathrm{M}}^{2}
$$

If this is not the case, and $n_{\mathrm{A}}^{0} \gg n_{\mathrm{M}}^{0}$, the system will often exhibit pseudo-first order kinetics, as $n_{\mathrm{A}} \approx n_{\mathrm{A}}^{0}$ throughout the approach to equilibrium. Thus Equation (1.31) 
becomes:

$$
\frac{d n_{\mathrm{A}-\mathrm{M}}}{d t}=k_{1}^{\prime} n_{\mathrm{M}}
$$

where $k_{1}^{\prime}=k_{1} n_{\mathrm{A}}^{0}$ and is a constant. The same consideration applies in cases where $n_{\mathrm{M}}^{0} \gg$ $n_{\mathrm{A}}^{0}$.

It is also possible to express these rate laws as the rate or approach to equilibrium, namely:

$$
\frac{d n_{\mathrm{A}-\mathrm{M}}}{d t}=k_{E q}\left(n_{\mathrm{M}_{E q}} n_{\mathrm{M}}\right)
$$

for the first order process, and

$$
\frac{d n_{\mathrm{A}-\mathrm{M}}}{d t}=k_{E q}\left(n_{\mathrm{M}_{E q}} n_{\mathrm{M}}\right)^{2}
$$

for the second order process. In this case, $k_{E q}$ represents the rate of approach towards equilibrium $\left(k_{1}-k_{-1}\right)$, while $n_{\mathrm{M}_{E q}}$ represents the amount of $\mathrm{M}$ in solution at equilibrium.

\subsection{Nanostructured Calcium Silicate}

Nanostructured Calcium Silicate (NCS) is a proprietary material reported recently by Johnston, McFarlane and Borrmann ${ }^{20,21}$ and discussed in more detail by McFarlane. ${ }^{22}$ The material consists of nanometre-size platelets stacked semi-randomly, forming aggregate particles of a few micrometres in size. The microstructure of the material is similar to the macrostructure of gypsum desert rose, as can be seen in Figure 1.1. Despite this microstructure, no significant long-range order exists within the material; it is largely $\mathrm{X}$ ray amorphous. However some broad peaks are present in the X-ray powder diffraction pattern of NCS. ${ }^{22}$ Such peaks, common to many calcium silicate hydrates, are indicative of two-dimensional ordering within the material. ${ }^{23}$ The material's microstructure imparts a large, highly accessible surface area, typically on the order of $400-600 \mathrm{~m}^{2} \mathrm{~g}^{-1}$, and an oil sorption capacity in excess of $600 .^{\dagger}$ The material also has a high brightness. NCS

\footnotetext{
Oil sorption, an approximate measure of pore volume, is reported as mass oil sorbed by the material on a wt $\%$ basis, thus an oil sorption of 600 would be reported if one gram of material sorbed six grams of oil. ${ }^{24}$
} 
typically has a high ISO brightness in excess of $88 \%^{\dagger}$ and a whiteness greater than 97. These characteristics have seen the material employed as a filler material in papers, as an aid for reducing print-through and ink-spreading, as well as contributing to the optical properties of the paper. ${ }^{21}$

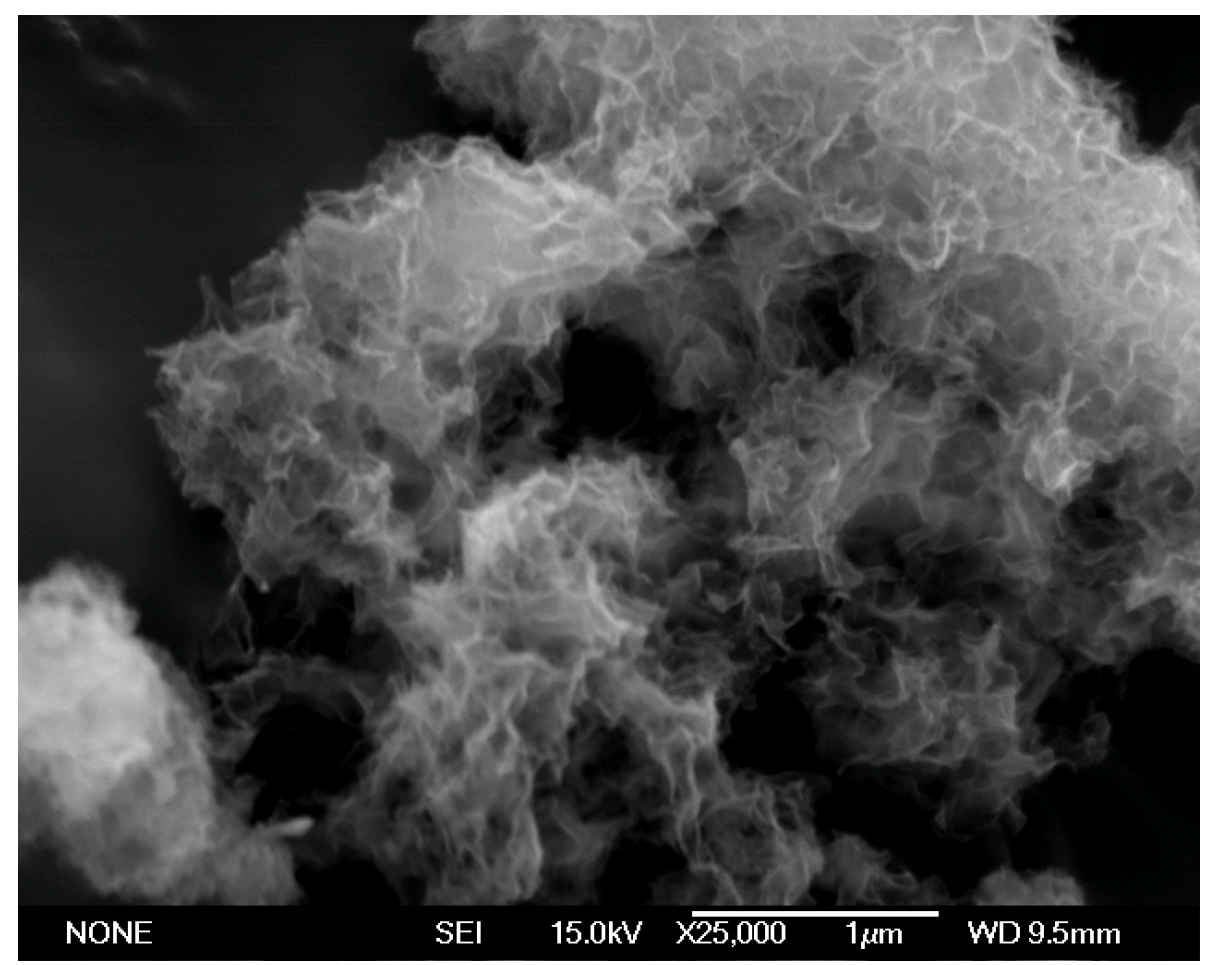

Figure 1.1 Scanning electron micrograph of nanostructured calcium silicate showing its porous microstructure.

\subsubsection{Structure}

From a chemical point of view, it is believed that the structure of the NCS consists of chains of connected $\mathrm{SiO}_{4}^{4-}$ tetrahedra, with these backbones bridged by $\mathrm{Ca}^{2+}$ ions. The material, being amorphous, does not have a defined chemical formula. From a typical elemental composition of the material, given in Table 1.4, its formula may be approximated by $\mathrm{Ca}_{0.8} \mathrm{SiO}_{2.8} \cdot 2 \mathrm{H}_{2} \mathrm{O}$. Solid state ${ }^{1} \mathrm{H}$ - and ${ }^{29} \mathrm{Si}-\mathrm{MAS}-\mathrm{NMR}$, X-ray Photoelectron Spectroscopy, X-ray Fluorescence and ion-beam spectroscopic studies of the material have demonstrated the presence of surface-bound hydroxyl groups and physisorbed water in the material, leading to the more appropriate formula $\mathrm{Ca}_{\mathrm{w}} \mathrm{SiO}_{\mathrm{x}}(\mathrm{OH})_{\mathrm{y}} \cdot \mathrm{zH}_{2} \mathrm{O}$, where:

\footnotetext{
${ }^{\dagger}$ Reported as reflectance at $457 \mathrm{~nm}^{25}$

${ }^{\ddagger}$ As measured on the $\mathrm{L}^{*}$ axis $\left(\right.$ white $=100$, black $=0$ ) of the CIELAB L*, $\mathrm{a}^{*}, \mathrm{~b}^{*}$ colour space. ${ }^{26}$
} 
- $\mathrm{w}=0.05-1^{\dagger}$

- $\mathrm{x}=2.05-3$

- $\mathrm{y}=0-0.5$

- $\mathrm{z}=1.4-2.5$

Table 1.4 Typical elemental composition of NCS as determined by XRF. ${ }^{27}$

\begin{tabular}{lr}
\hline Analyte & Percent by mass \\
\hline $\mathrm{SiO}_{2}$ & 45.52 \\
$\mathrm{CaO}$ & 38.05 \\
$\mathrm{LOI}^{a}$ & 14.45 \\
Other $^{b}$ & 1.58 \\
\hline Total & 99.60 \\
\hline
\end{tabular}

${ }^{a}$ Loss on ignition at $1000^{\circ} \mathrm{C}$.

${ }^{b}$ Minor contribution from $\mathrm{Al}_{2} \mathrm{O}_{3}, \mathrm{Fe}_{2} \mathrm{O}_{3}, \mathrm{MgO}, \mathrm{Na}_{2} \mathrm{O}, \mathrm{K}_{2} \mathrm{O}, \mathrm{TiO}_{2}, \mathrm{P}_{2} \mathrm{O}_{5}$

The structure is represented schematically in Figure 1.2.
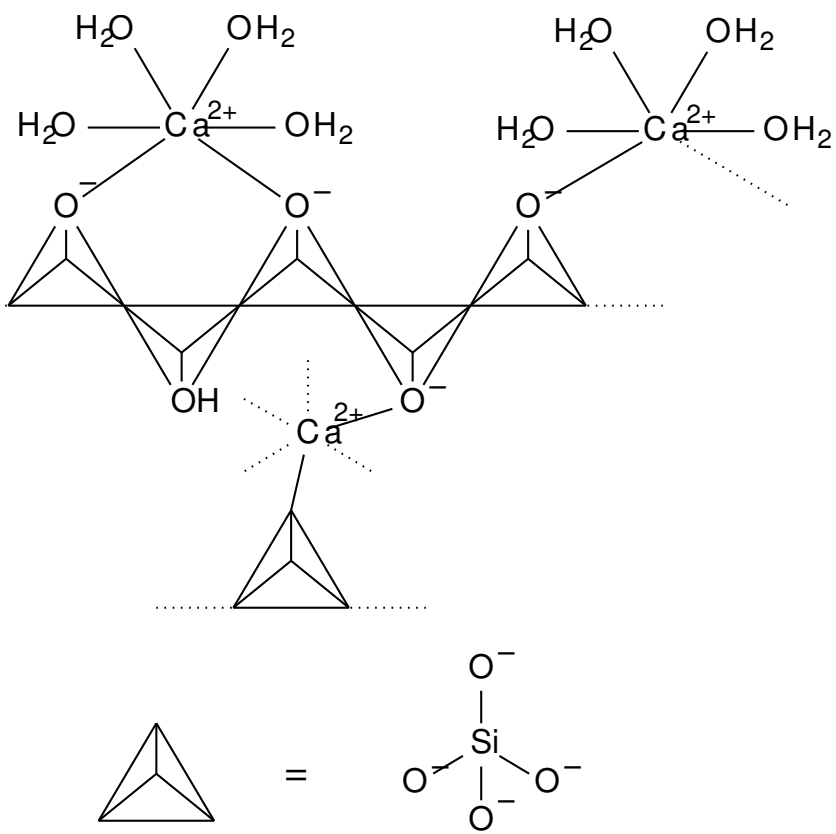

Figure 1.2 Suggested formula and schematic of nanostructured calcium silicate, showing the chain-like nature of the material. Only selected atoms in the $\mathrm{SiO}_{4}^{4-}$ tetrahedra are shown.

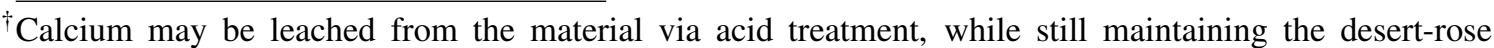
structure. 
At high temperatures (in excess of $720^{\circ} \mathrm{C}$ ), the NCS is converted to wollastonite $\left(\mathrm{CaSiO}_{3}\right),{ }^{\dagger}$ as evidenced by $\mathrm{X}$-ray powder diffraction studies. ${ }^{28}$ This leads to the assumption that the structure of NCS shares some similarities with the chain-like structure found in wollastonite. Evidence for the chain-like structure is also seen in ${ }^{29} \mathrm{Si}-\mathrm{NMR}$ studies. ${ }^{22,28}$

NCS is synthesised via a precipitation reaction from aqueous calcium ions and silica precursors. As such, the chain-like nature of the silicate backbone within NCS poses some problems during the drying step. As the material is dried, the large surface area of the material leads to a large liquid-air interface, resulting in a high surface tension. NCS does not form a rigid skeletal framework structure like those possessed by minerals such as halloysite, pillared clays or zeolites. Thus, the large surface tension acts to draw the material's platelets together, decreasing its surface area and pore volume. Drying the material also leads to a change in the coordination environment of the $\mathrm{Ca}^{2+}$ ions, most likely substituting coordinated water for $\mathrm{SiO}$ or $\mathrm{SiOH}$ groups. This means that drying NCS can irreversibly lead to a loss of its microstructure. Three different approaches have been developed by McFarlane ${ }^{22}$ to overcome this problem:

- The first approach is to reduce the surface tension of the solvent. Water, due to its extensive hydrogen bonding, has a very large surface tension. If, in a filtration step immediately prior to drying, the water in the system is displaced by a solvent having a lower surface tension, the collapse of the structure is mitigated. Solvents typically employed for this purpose are ethanol or 2-ethoxyethanol. The surface tension of these solvents is given in Table 1.5. If the dry material is subsequently wetted with water however, the phenomenon of structure collapse upon drying reasserts itself.

When 2-ethoxyethanol washing is used, a small portion of the solvent remains coordinated to $\mathrm{Ca}^{2+}$ on the NCS surface. This can slightly reduce the chemical reactivity of the NCS surface when compared to the unmodified material. Coordination of the 2-ethoxyethanol also means that collapse of the NCS structure is retarded to a greater degree than when ethanol washing is used, despite the lower surface tension

${ }^{\dagger}$ Silica is also formed, thus maintaining the $\mathrm{Ca}: \mathrm{Si}$ ratio within the system
$\left(\mathrm{Ca}_{0.8} \mathrm{SiO}_{2.8} \cdot 2 \mathrm{H}_{2} \mathrm{O} \stackrel{\Delta}{\longrightarrow} 0 \cdot 8 \mathrm{CaSiO}_{3}+0 \cdot 2 \mathrm{SiO}_{2}+2 \mathrm{H}_{2} \mathrm{O} \uparrow\right)$.


of the latter. The 2-ethoxyethanol remains coordinated even after prolonged drying of the material at $110^{\circ} \mathrm{C}$. When such materials are heated in air to $600^{\circ} \mathrm{C}$, the 2 ethoxyethanol treated material exhibits a mass loss approximately $10 \%$ greater than that of the untreated material.

Table 1.5 Surface tension of various solvents in contact with air at $25^{\circ} \mathrm{C}$ as reported by Lide. ${ }^{29}$

\begin{tabular}{|c|c|}
\hline Solvent & Surface tension/ $10^{-3} \mathrm{~N} \mathrm{~m}^{-1}$ \\
\hline Water & 71.99 \\
\hline Ethanol & 21.97 \\
\hline 2-Ethoxyethanol & 28.35 \\
\hline
\end{tabular}

- The second approach commonly employed is to reinforce the material. This is done by condensing a layer of silica between adjacent platelets of the material. If an aqueous silicate solution is introduced to a slurry of NCS, the $\mathrm{H}_{(4-n)} \mathrm{SiO}_{4}^{\mathrm{n}-}$ ions will preferentially sorb in the acute angle formed at the interface of two platelets as this minimises the surface tension of the system. Sorption of $\mathrm{H}_{(4-n)} \mathrm{SiO}_{4}^{\mathrm{n}-}$ on the platelet surfaces also occurs. Subsequent acidification of the system causes the silicate ions to condense to a silica layer. This mechanically reinforces the system, retarding structure collapse upon drying. The reinforced NCS can be wetted and dried several times without causing a significant reduction of its surface area.

Reinforcement of the NCS results in a slightly reduced specific surface area, as extra mass has been added to the system. From a chemical standpoint, the procedure also reduces the mole ratio of calcium within the system.

- The third method involves acid treatment of the as-made NCS slurry. The $\mathrm{Ca}^{2+}$ in the NCS structure ion exchanges with $\mathrm{H}^{+}$, leading to the formation of additional silanol groups. Adjacent silanol groups may then condense, reducing the polarity, and hence surface tension, of the NCS material. This method leads to a loss of chemical functionality in the NCS material. Like the reinforcement method, the material may be wetted and dried several times with little observed change in the surface properties.

However, this method is wasteful of reagents, as most of the calcium is leached from the NCS. This leads to a significant decrease in the final mass of product. A 
typical $\mathrm{Ca}: \mathrm{Si}$ ratio in the $\mathrm{pH}$ adjusted material is $0.05: 1$, compared to $0.8: 1$ prior to $\mathrm{pH}$ adjustment. ${ }^{22}$ As such, this technique is rarely used as the primary method for retarding structural collapse, but rather when it is also desirable to produce a material with a low calcium content.

\subsubsection{Physical Properties}

Recovery of the NCS material by filtration is difficult, due to its high, readily accessible surface area and small particulate size. Even under sustained vacuum filtration, the filter-cake will retain a large quantity of water. A typical NCS filter-cake will contain only 5-15 wt $\%$ solids $^{\dagger}$ (in other words, $85-95 \mathrm{wt} \%$ of the material is water). The material is thixotropic, meaning that a stirred, aqueous suspension of NCS will form a solid mass upon standing. The apparently solid material can be returned to a thin slurry upon additional stirring however. This thixotropic nature is probably caused by hydrogen bonding between adjacent NCS particulates. During filtration, the small particle size and thixotropic nature of the material lead to the formation of a small, dense layer of NCS material at the bottom of the filtration device; excess solution in the upper parts of the filtration device is removed very slowly.

While an inefficient filtration process is not very problematic on a laboratory scale, such a time-consuming step is highly undesirable on an industrial scale — the extra time required for filtration makes the NCS recovery process unsuitable. For this reason, part of this study involves incorporation of magnetic centres within the NCS structure to aid separation of the material from solution. This is covered in more detail in Chapter 4.

\subsubsection{Chemistry}

The presence of labile calcium ions at or near the surface of the material provides the basis for much of the solution based chemistry of NCS. When NCS is permitted to equilibrate with water, the $\mathrm{pH}$ of the system increases until a value of approximately 10.5 is reached.

\footnotetext{
${ }^{\dagger}$ When compared to the dry mass, after drying at $110^{\circ} \mathrm{C}$.
} 
This occurs due to exchange of surface-bound $\mathrm{Ca}^{2+}$ with $\mathrm{H}^{+}$(from $\mathrm{H}_{2} \mathrm{O} \rightleftharpoons \mathrm{H}^{+}+\mathrm{OH}^{-}$), although exchange of $\mathrm{Ca}^{2+}$ with other ions, for example $\mathrm{Sr}^{2+}$, is also known. ${ }^{30}$

The release of $\mathrm{Ca}^{2+}$ by NCS when in contact with water occurs on a rather short time scale; achieving equilibrium after a few hours instead of time scales ranging from days to months for some of the more stable crystalline calcium silicates such as wollastonite. ${ }^{31}$ If NCS is reacted with acid, $\mathrm{Ca}^{2+}$ will be leached from the material, as has been mentioned above. If the addition of acid is undertaken slowly, nearly all calcium will be leached from the material when the equilibrated system reaches $\mathrm{pH}$ 6. At this point the material still retains much of its initial morphology. If excess acid is added to the system, formation of a gel-like silica phase bearing little resemblance to the initial material, results.

The surface accessible $\mathrm{Ca}^{2+}$ in the system means that potential complexes can be formed, if suitable ligand molecules are used. Borrmann et al. ${ }^{32}$ have previously demonstrated that NCS powder will sorb molecular iodine, through the formation of a $\mathrm{Ca}-\mathrm{I}$ complex. The iodine is retained in the system, even after sustained heating at $110^{\circ} \mathrm{C}$. The nature of the $\mathrm{Ca}-\mathrm{I}$ bonding has been studied by XPS, the results of which suggest that the iodine is present in the zero-valent state. However, a formal representation of the $\mathrm{Ca}-\mathrm{I}$ interactions could not be elucidated.

NCS also has the potential to sorb anionic species having a high affinity for $\mathrm{Ca}^{2+}$, of which phosphate is one example. Southam ${ }^{33-35}$ has performed extensive studies of the unmodified NCS for use as a sorbent for phosphate. The results of these studies are discussed in greater detail in Chapter 5, in which phosphate is used as a model sorbate to characterise the sorption properties of some $\mathrm{Fe}_{3} \mathrm{O}_{4}-\mathrm{NCS}$ composite materials.

\subsubsection{Conducting Polymer Derivatives}

In addition to its ability to sorb small ionic (phosphate) or molecular (iodine) species, the large surface area of NCS makes it potentially desirable as a substrate for materials having interesting electrochemical properties, such as conducting polymers. The conducting polymers, such as polyaniline or polypyrrole, are a class of organic polymers typified 
by extensive $\pi$-conjugation along the polymer backbone. This property makes them amenable to possess high conductivities upon the addition of suitable dopant materials. Their conductive nature was first reported by MacDiarmid et al. ${ }^{36,37}$ although several conducting polymers have been known for many years. For example, the electrochemical synthesis of polyaniline was first reported in $1862 .{ }^{38}$ While the materials themselves possess interesting electrical properties, they often have poor mechanical characteristics such as processability. For this reason, they are often incorporated into composite materials.

\subsection{Scope of the Thesis}

The structure of NCS provides a material having a high surface area and pore volume. These properties, along with the presence of chemically active calcium within the NCS structure make the material desirable as a potential sorbent material.

Although the use of NCS for the sorption of phosphate has previously been characterised, ${ }^{34}$ little is known about the use of this material as a sorbent for cationic species. Due to the basic nature of the NCS, ${ }^{22}$ it was assumed that the material should prove suitable as a sorbent for species exhibiting insoluble hydroxides.

This thesis characterises the sorption properties of NCS with respect to $\mathrm{Cu}^{2+}$. The copper ion was chosen as a model compound due to its similarities to many other, potentially toxic, cationic species and its propensity to form insoluble hydroxide precipitates. The kinetics of the sorption process are elucidated, and the nature of the sorbed product determined by a variety of instrumental techniques. The thixotropic nature of NCS precludes its use as an adsorbent material on an industrial scale due to problems associated with its recovery from solution following sorption. To overcome this problem, incorporation of magnetic minerals magnetite or maghemite into the silicate structure is undertaken. The sorption characteristics of the magnetic composite materials with respect to the copper and phosphate are determined, and the magnetic nature of the composite material is utilised to aid in its separation from solution following sorption. 
The high surface area and pore volume of the material are also utilised by employing NCS as a substrate for the conducting polymer polyaniline. The redox-active nature of the resulting composite material is also investigated in sorption studies of the oxyanion perrhenate, $\mathrm{ReO}_{4}^{-}$. 


\section{Chapter 2}

\section{Experimental}

\subsection{General}

The majority of the experimental work reported in this thesis was performed at Victoria University. Work concerning phosphate sorption was conducted during a research stay at the Water and Geotechnology Division of the Institute for Technical Chemistry at the Forschungszentrum Karlsruhe (FZK), Karlsruhe, Germany. As such, reagents and equipment used for work associated with phosphate sorption differ from those used for the remainder of this work.

Reagents used for phosphate sorption experiments were obtained from Merck as analytical reagent grade and used as received. Specifically, this includes all chemicals listed in the phosphate section. Reagents used to synthesise NCS materials for phosphate sorption tests were obtained from Merck (as AR grade), with the exception of magnetite powder, which was purchased from Bayer. Buffer solutions used as calibrants for pH determination were obtained from VWR Prolabo, France. Reagent water for the preparation of solutions and for dilutions was purified with a MilliQ system (Millipore), such that it had a resistivity greater than $18 \mathrm{M} \Omega \mathrm{cm}^{-1}$.

For NCS materials employed in other investigations, such as for copper and rhenium sorption studies, calcium hydroxide was obtained from LobaChemie (AR grade), sodium silicate was obtained from Orica Chemicals ( $\mathrm{N}$ grade) and hydrochloric acid was obtained from JaSol. Water for all preparations was distilled prior to use. Magnetite powder, for use in preparing composite materials, was obtained from Bayer. For the preparation of PANI composite materials, ammonium peroxydisulfate was sourced from Avocado chemicals. Aniline was purchased from Sigma-Aldrich and distilled prior to use. It was stored in an amber bottle at $4{ }^{\circ} \mathrm{C}$ and was redistilled, if it became discoloured. Other than aniline, all chemicals were used as received. 
For sorption tests (other than for phosphate sorption), and for elemental analysis, analytical grade reagents were used for all work. Chemicals for this work were obtained from a variety of sources:

- Copper chloride from Ajax,

- Nitric acid from APS,

- Calcium carbonate from BDH,

- Rhenium spectroscopy standards from Fluka,

- Hydrochloric acid and sulfuric acid from LabScan,

- $\mathrm{pH}$ buffer solutions from Pure Science,

- Copper nitrate and iron sulfate from Riedel-de-Haën,

- Potassium perrhenate and strontium chloride from Sigma-Aldrich.

\subsubsection{Chemical Analytical Techniques}

\subsubsection{Phosphate Analysis}

Quantitative determination of phosphate was undertaken with a molybdenum blue method. The method used was an in-house modification (by FZK) of other published methods, and employed bismuth as a stabilising agent. ${ }^{10,39}$ The following reagents were used:

- SMOR (Molybdic Acid Reagent): Ammonium molybdate, $\left(\mathrm{NH}_{4}\right)_{6} \mathrm{Mo}_{7} \mathrm{O}_{24} \cdot 4 \mathrm{H}_{2} \mathrm{O}$ (9.15 g), was dissolved in approximately $700 \mathrm{~cm}^{3}$ of $\mathrm{H}_{2} \mathrm{O}$. In a separate beaker, $\mathrm{Bi}\left(\mathrm{NO}_{3}\right)_{3} \cdot 5 \mathrm{H}_{2} \mathrm{O}(1.5 \mathrm{~g})$ was dissolved in $191 \mathrm{~cm}^{3}$ of concentrated $\mathrm{HNO}_{3}(65 \%$, $14 \mathrm{~mol} \mathrm{dm}^{-3}$ ) and then slowly added to the molybdate solution. The resulting solution was allowed to cool and its volume adjusted to $1000 \mathrm{~cm}^{3}$. 
- RED (Reducing Agent): 1-Amino-2-naphthol-4-sulfonic acid, ANS, (0.750 g), $\mathrm{Na}_{2} \mathrm{SO}_{3}$ (41.92 g), and $\mathrm{Na}_{2} \mathrm{~S}_{2} \mathrm{O}_{5}(69.85 \mathrm{~g})$, were measured out. All of the ANS and approximately one half of the $\mathrm{Na}_{2} \mathrm{~S}_{2} \mathrm{O}_{5}$ were ground to a fine powder in a mortar. The remaining solids were dissolved in approximately $800 \mathrm{~cm}^{3} \mathrm{H}_{2} \mathrm{O}$, then the ground powder mixture was added to the solution. Once dissolved, the resulting solution was adjusted to a final volume of $1000 \mathrm{~cm}^{3}$.

- Both reagents were stored at $4{ }^{\circ} \mathrm{C}$ in glass bottles (the $\mathrm{RED}$ reagent in an amber bottle). They were discarded if they became coloured. Before use, the reagents (and any standards or samples) were allowed to warm to room temperature.

To analyse for phosphate, a series of standards containing $0.0,0.2,0.4,0.6,0.8$, $1.0 \mathrm{mg} \mathrm{P} \mathrm{dm}^{-3}$ were prepared daily from a stock solution (prepared weekly) containing $10 \mathrm{mg} \mathrm{P} \mathrm{dm}{ }^{-3}$ (prepared from $\mathrm{Na}_{2} \mathrm{HPO}_{4}$ ). Aliquots of any samples to be analysed were placed into $25 \mathrm{~cm}^{3}$ volumetric flasks such that the final phosphate concentration was in the range covered by the standards. Water was added to bring the total volume to approximately $15 \mathrm{~cm}^{3}$. Following this, $2.5 \mathrm{~cm}^{3}$ SMOR was added, the solution mixed gently, and $0.5 \mathrm{~cm}^{3}$ RED added. All flasks were made to volume with water and the molybdenum blue colour was allowed to develop for 15 minutes. The absorbance of standards and samples was measured in disposable cuvettes $(1 \mathrm{~cm}$ width) at a wavelength of $690 \mathrm{~nm}$ using a WTW Mikroprozessor Photometer MPM 1500 (WissenschaftlichTechnische Werkstätten GmbH, Weilheim). A calibration chart was prepared and used to determine the phosphate concentration of the samples. The absorbance was found to obey Beer's Law, having a linear response, with an $R^{2}$ value typically greater than 0.995 .

Typically, the amount of dissolved reactive phosphorus (DRP) was determined, in which case the samples were filtered with $0.45 \mu \mathrm{m}$ cellulose membranes (Millipore) before analysis. In some cases total reactive phosphorus (TRP) was quantified, in which case the samples were not filtered before analysis. Occasionally, the concentration of total phosphorus (TP) or total dissolved phosphorus (TDP) was determined. In this case, the unfiltered (TP) or filtered (TDP) sample was digested with concentrated $\mathrm{HNO}_{3}$ in a Teflon lined bomb at $100^{\circ} \mathrm{C}$ overnight. Typically $0.25 \mathrm{~cm}^{3} \mathrm{HNO}_{3}$ and either $5 \mathrm{~cm}^{3}$ or $0.2 \mathrm{~g}$ 
sample were digested in a $30 \mathrm{~cm}^{3}$ bomb before being diluted to $100 \mathrm{~cm}^{3}$. At least one standard was similarly treated when TDP or TP were determined.

\subsubsection{Spectrophotometric Determination of Iodine}

Iodine was quantified by spectrophotometric determination of the iodine-starch complex. Due to the poor stability of $\mathrm{I}_{3}^{-}$in solution, standards were prepared in situ from $\mathrm{KIO}_{3}$ according to Equation (2.1):

$$
\begin{aligned}
& \mathrm{IO}_{3}^{-}+6 \mathrm{H}^{+}+5 \mathrm{I}^{-} \longrightarrow 3 \mathrm{I}_{2}+3 \mathrm{H}_{2} \mathrm{O} \\
& \mathrm{I}_{2}+\mathrm{I}^{-} \rightleftharpoons \mathrm{I}_{3}^{-}
\end{aligned}
$$

A stock solution of $\mathrm{KIO}_{3}$ was prepared by dissolving $1.1242 \mathrm{~g}$ of the salt with water in a $100 \mathrm{~cm}^{3}$ flask, providing a $52.53 \mathrm{mmol} \mathrm{dm}{ }^{-3}$ solution. Solutions of $\mathrm{NaI}\left(1 \mathrm{~mol} \mathrm{dm}^{-3}\right)$, $\mathrm{H}_{2} \mathrm{SO}_{4}\left(1 \mathrm{~mol} \mathrm{dm}{ }^{-3}\right)$ and starch $\left(1 \mathrm{~g} \mathrm{dm}^{-3}\right)$ were also prepared. The starch solution was replaced it it became turbid. When fully reacted according to $(2.1), 1 \mathrm{~cm}^{3}$ of the $\mathrm{IO}_{3}^{-}$ standard provided an equivalent mass of $0.4000 \mathrm{~g}$ elemental iodine. Serial dilutions of this standard were made to provide a working standard with an equivalent $\mathrm{I}_{2}$ concentration of $20 \mathrm{mg} \mathrm{dm}^{-3}$.

Standard solutions with equivalent $\mathrm{I}_{2}$ concentrations of $10,7.5,5.0,2.5$ and $0 \mathrm{mg} \mathrm{dm}^{-3}$ were prepared by transferring aliquots of the working standard to $25 \mathrm{~cm}^{3}$ volumetric flasks. Aliquots of the samples to be analysed were likewise taken. To the flasks was added $1 \mathrm{~cm}^{3}$ each of the $\mathrm{NaI}$ and $\mathrm{H}_{2} \mathrm{SO}_{4}$ solutions and the flasks made nearly to volume with water. Prior to the final volume adjustment, $0.1 \mathrm{~cm}^{3}$ of the starch solution was added to each flask. The absorbance of the iodine-starch complex was measured (using $1 \mathrm{~cm}$ wide quartz cuvettes) at a wavelength of $352 \mathrm{~nm}$ on a Cary Scan 100 spectrophotometer. The analytical wavelength was chosen by recording the visible spectrum of the iodinestarch complex, so as to determine to the wavelength of maximum absorption, prior to quantitative analysis. 


\subsubsection{Quantification of Metal Species}

Quantification of metal species was undertaken on a GBC906AA flame atomic absorption spectrometer. Prior to analysis, all samples were filtered through $0.45 \mu \mathrm{m}$ cellulose membranes in order to remove any suspended solids. Calibration was performed with a minimum of 4 standard solutions. Each standard and sample was analysed in triplicate by the instrument, which also automatically prepared calibration curves. Where necessary, samples were diluted such that the analyte concentration was in the range covered by the standards. During the analysis, the calibration was checked every 10 samples by reanalysing a calibration standard, a blank solution and a sample. Analysis conditions used were those recommended by the instrument manufacturer, ${ }^{40}$ which were consistent with other sources. ${ }^{10}$ The instrument operating parameters, for specific elements, are given in Table 2.1.

\subsubsection{Instrumental Uncertainties}

Where quantification of metal species was undertaken by AA, the instrument analysed each sample in triplicate and calculated the percentage relative standard deviation of the result:

$$
\% R S D=\frac{\sigma_{y}}{\bar{y}} \times 100
$$

This value was used to calculate the standard deviation for the measured concentration. For analytes quantified by UV/visible spectroscopy, namely phosphate and iodine, the standard error in the linear regression fit for the calibration data was applied to all samples. For determination of $\mathrm{pH}$, an uncertainty of \pm 0.05 of a $\mathrm{pH}$ unit was used, being the instrumental accuracy specified by the manufacturers (see Section 2.1.2.7). 


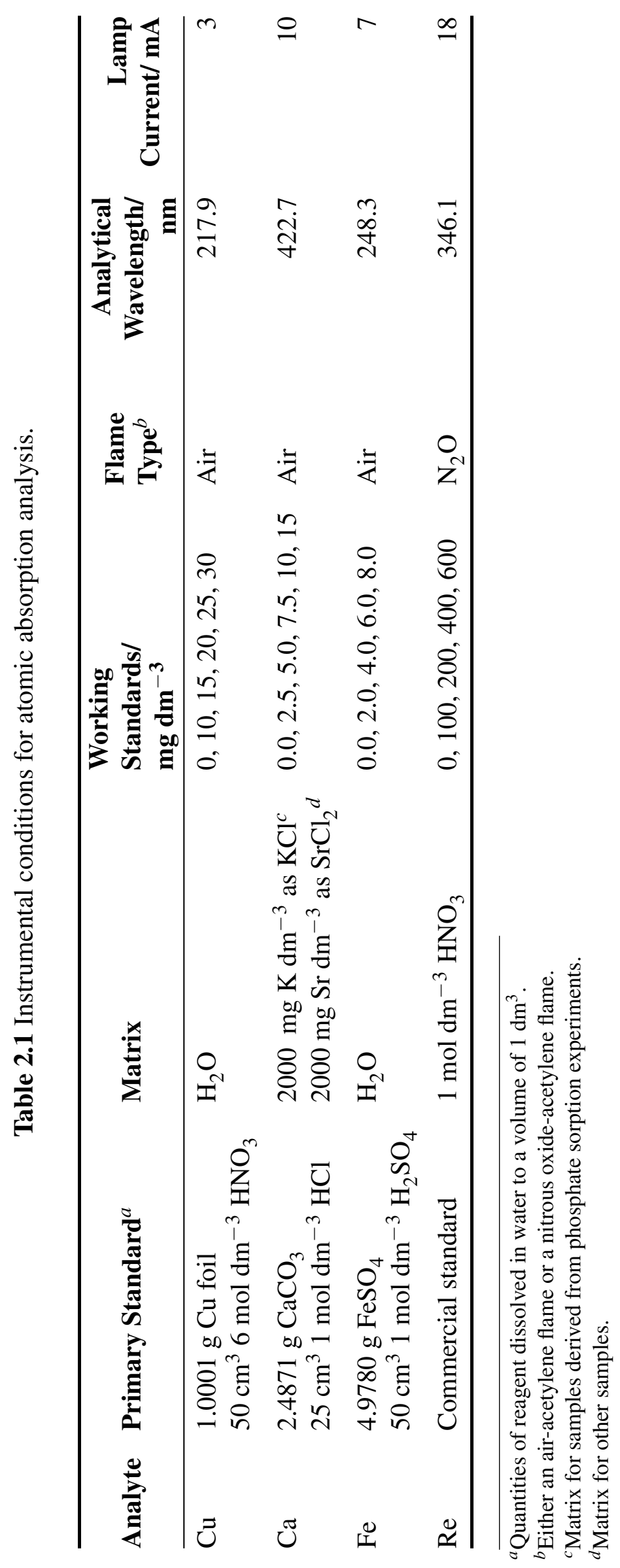




\subsubsection{Other Instrumental Techniques}

\subsubsection{X-ray Analysis}

Powder X-ray diffraction patterns were collected using a Phillips PW 3710 MPDcontrolled diffractometer. Typically, scans were recorded through an angular range of $6-70^{\circ} 2 \theta$ at a scan speed of $0.020^{\circ} 2 \theta \mathrm{s}^{-1}$ using the $\mathrm{Cu} \mathrm{K}_{\alpha}$ spectral line. In cases where the XRD pattern was assigned to specific crystalline phases, reference patterns were taken from the ICDD Powder Diffraction File Version $4+{ }^{41}$ and were of either Star or Indexed quality.

\subsubsection{Particle Size Analysis}

Particle size distributions were determined using a Malvern Mastersizer Hydro 2000 MU. Preferentially, the particle size distribution of NCS samples were determined from samples in the form of a slurry or filter cake. Ultrasonication was performed for 30 seconds to ensure complete dispersion of the sample prior to data collection. Raw data were processed by the instrument software such that a number-weighted size distribution was calculated, based on the average of three measurements.

\subsubsection{Electron Microscopy}

Electron microscopy was performed on a JEOL JSM 6500F field emission scanning electron microscope. Prior to analysis, samples were adhered to carbon tape on aluminium stubs and sputter-coated with a $4 \mathrm{~nm}$ thick Pt layer. The same instrument was used to record both electron micrographs and energy dispersive X-ray spectra (EDS). In cases where the Pt M spectral lines overlapped with the analyte of interest (typically Re), a carbon sputter-coater was used in place of the Pt one. 


\subsubsection{Thermogravimetric Analysis}

Thermogravimetric analysis was undertaken on a Shimadzu TGA-50 instrument. Typically, samples were heated at a rate of $10{ }^{\circ} \mathrm{C} \mathrm{min}^{-1}$, in a stream of dry air at a flow rate of $50 \mathrm{~cm}^{3} \mathrm{~min}^{-1}$. Platinum sample pans were used for analysis. Samples were held at a temperature of $105^{\circ} \mathrm{C}$ until a constant mass was achieved. This typically took 15 to 30 minutes. Once a constant initial mass had been established, heating was resumed. In cases where NCS samples were being analysed, the samples were heated to a temperature of $950^{\circ} \mathrm{C}$. Once the maximum temperature was reached, it was maintained for 5 minutes, to ensure that a constant mass was achieved.

\subsubsection{Surface Area Analysis}

Single point BET surface area determinations were undertaken with the use of a Mircomeritics FlowSorb II 2300 instrument, using a gas mixture of 29.1 vol $\% \mathrm{~N}_{2}$ in $\mathrm{He}$. Due to the propensity of NCS for sorbing water, samples were degassed for 1 hour under the $\mathrm{N}_{2}$ stream at $105^{\circ} \mathrm{C}$ prior to analysis. The instrument measured the volume of nitrogen adsorbed or desorbed based on differential gas flow rates across the sample. Instrument calibration was undertaken by injection of $1 \mathrm{~cm}^{3} \mathrm{~N}_{2}$ gas into the machine, providing a differential flow rate equivalent to a desorption value of $2.84 \mathrm{~m}^{2}$ at $298 \mathrm{~K}$. Specific surface area was calculated with a modified form of the BET equation recommended by the manufacturer, ${ }^{42}$ using the measured nitrogen desorption value. ${ }^{\dagger}$

$$
\text { Specific Surface Area }=\frac{C}{m} \times \frac{298 \mathrm{~K}}{T_{a}} \times \frac{P_{a}}{760 \mathrm{~mm} \mathrm{Hg}} \times \frac{1-\frac{x_{\mathrm{N}_{2}} P_{a}}{P_{a}+15 \mathrm{~mm} \mathrm{Hg}}}{0.706}
$$

Where:

$C=$ integrator counts (surface area) from nitrogen desorption $/ \mathrm{m}^{2}$

$m=$ sample mass $/ \mathrm{g}$

$T_{a}=$ ambient temperature/ $\mathrm{K}$

$P_{a}=$ ambient pressure/ $\mathrm{mm} \mathrm{Hg}$

${ }^{\dagger}$ The instrument calculated this value based on the volume of nitrogen desorbed by the sample on warming it from $77 \mathrm{~K}$ to room temperature. 
$x_{\mathrm{N}_{2}}=\operatorname{vol} \% \mathrm{~N}_{2}, 29.1 \%$

\subsubsection{Cyclic Voltammetry}

Cyclic voltagramms were recorded on an AMEL 7050 potentiostat. Working- and counter-electrodes were made from glassy carbon, and a silver/silver chloride electrode $\left(\mathrm{Ag}(\mathrm{s})|\mathrm{AgCl}(\mathrm{s})| \mathrm{Cl}^{-}(\mathrm{aq})\right)$ was used as the reference electrode. Saturated $\mathrm{KClO}_{4}$ solution was used as the electrolyte, chosen in preference to $\mathrm{KCl}$ as the former allowed for a greater electrode potential range. Measurements were made at room temperature (approximately $25^{\circ} \mathrm{C}$ ) — the temperature was not otherwise controlled. Scans were recorded for 10 cycles between $-1.5 \mathrm{~V}$ and $+1.2 \mathrm{~V}$ relative to the reference electrode. The first five cycles at any given scan speed were permitted for the system to stabilise. Hence, the data from these cycles were ignored - only data from the final five scans were used. Multiple scans were recorded, at scan rates of $100 \mathrm{mV} \mathrm{s}^{-1}, 50 \mathrm{mV} \mathrm{s}^{-1}$, and $20 \mathrm{mV} \mathrm{s}^{-1}$.

\subsubsection{7 pH}

For solutions associated with phosphate sorption work, $\mathrm{pH}$ determinations were made with a WTW pH340i meter equipped with a SenTix 41 combined $\mathrm{pH}$-temperature electrode. A three-point calibration was performed with $\mathrm{pH}$ 4.00, 7.00 and 10.00 buffers. Typically, the response from the meter was found to be within $5 \%$ of the Nernst potential $\left(59.1 \mathrm{mV} \mathrm{pH}^{-1}\right)$. The auto-read function of the meter was employed, meaning that a reading was not taken until the output signal from the $\mathrm{pH}$ electrode was stable to \pm 0.01 $\mathrm{pH}$ units for 30 seconds.

For other work, $\mathrm{pH}$ was determined using a HANNA Instruments 5521 meter or a EuTech Instruments CyberScan pH 11 meter. For the EuTech meter, a three point calibration was performed using $\mathrm{pH} 4.01,7.00$ and 10.01 buffers. Two point calibration was used with the HANNA meter, using the pH 7.00 buffer and either the 4.01 or 10.01 buffer. These meters lacked an auto-read function. However, like the WTW meter, $\mathrm{pH}$ readings were not taken 
until the signal from the $\mathrm{pH}$ electrode was stable to $\pm 0.01 \mathrm{pH}$ units for 30 seconds.

\subsubsection{UV/ Visible Spectroscopy}

UV/visible absorption spectroscopy was performed on a Cary Scan 100 instrument (Varian) operating in double beam mode. Samples were transferred into quartz cuvettes (Roth) of $1 \mathrm{~cm}$ width and spectra were recorded between 200-800 nm, at a scan speed of $200 \mathrm{~nm} \mathrm{~min}{ }^{-1}$. The spectral bandwidth of the instrument was set to $2 \mathrm{~nm}$.

\subsubsection{Reflectance Spectroscopy}

Two instruments were used to record reflectance spectra - a HunterLabs ColorQuest C5160 or a Cary Scan 100, with a reflective sphere accessory fitted. The Cary instrument, while having a superior spectral resolution $(2 \mathrm{~nm}$, versus $10 \mathrm{~nm}$ on the HunterLabs instrument), could not be accurately calibrated to provide absolute reflectance. For qualitative analysis however, the Cary instrument was deemed more appropriate. Samples to be measured were initially ground to a fine powder in an agate mortar then pressed into a homemade sample holder, similar in style to sample holders commonly employed for powder XRD measurement.

\subsubsection{X-Ray Photoelectron Spectroscopy}

XPS measurements were undertaken on a Kratos Axis Ultra XPS instrument located at Auckland University. The $\mathrm{Al} \mathrm{K}_{\alpha} \mathrm{X}$-ray source was provided by an filament operating at $10 \mathrm{~mA}$ and $15 \mathrm{kV}$. Prior to analysis, samples were mounted on carbon tape, which was used as the internal standard, with the $\mathrm{C} 1 \mathrm{~s}$ peak adjusted to a binding energy of $285 \mathrm{eV}$. Data analysis was performed with the fity $k$ program, ${ }^{43}$ which was used to model the experimental data with Gaussian-Lorentzian peaks having 20\% Lorentzian character. The function is given in Equation (2.5) where $h$ is the peak height, $c$ its centre and $w$ its half width at half median height. A Shirley background ${ }^{44}$ was subtracted from the data 
prior to peak fitting:

$$
f(x)=h\left[0.8 \exp \left(-\ln (2)\left(\frac{x-c}{w}\right)^{2}\right)+\frac{0.2}{1+\left(\frac{x-c}{w}\right)^{2}}\right]
$$

\subsubsection{Magnetometry}

Magnetisation of $\gamma-\mathrm{Fe}_{2} \mathrm{O}_{3}$-NCS composite materials was measured on a Alternating Gradient Magnetometer, model 2900 (Princeton Measurements Corporation). A known mass of sample (a few milligrams) was packed into a glass capillary tube, the ends of which were sealed with glue to prevent sample movement within the tube. Silicone grease was used to mount the sample on the instrument's sample probe. Magnetisation curves were recorded at a scan rate of $8 \mathrm{kA} \mathrm{m}^{-1} \mathrm{~s}^{-1}$ between $+800 \mathrm{kA} \mathrm{m}^{-1}$ and $-800 \mathrm{kA} \mathrm{m}^{-1}$.

\subsection{Synthesis of Calcium Silicate and Its Derivatives}

\subsubsection{General Synthetic Procedure}

NCS was prepared using a batch process. Typically batches of between $200-500 \mathrm{~cm}^{3}$ were prepared. The following relates to the preparation of a sample at a reaction volume of $500 \mathrm{~cm}^{3}$, giving approximately $10 \mathrm{~g}$ (dry basis) of calcium silicate:

In a plastic beaker, $\mathrm{Ca}(\mathrm{OH})_{2}(78.1 \mathrm{mmol}, 5.79 \mathrm{~g})$, was suspended in $250 \mathrm{~cm}^{3}$ of $0.174 \mathrm{~mol} \mathrm{dm}^{-3} \mathrm{HCl}$ under vigorous mixing provided by a mechanical overhead stirrer (either a Heidolph RZR 1 or an IKA-Werke Eurostar Digital). In a separate plastic beaker sodium silicate (15.91 $\mathrm{g}$ of $27 \mathrm{wt} \% \mathrm{SiO}_{2}, 71.5 \mathrm{mmol}$ ) was diluted to $250 \mathrm{~cm}^{3}$ with $\mathrm{H}_{2} \mathrm{O}$. This provided precursor solutions with the following equivalent concentrations: ${ }^{\dagger}$

- $[\mathrm{Ca}]=0.312 \mathrm{~mol} \mathrm{dm}^{-3}$

‘In the case of calcium, this 'concentration' is given as if the calcium hydroxide particles were fully dissolved, which is not the case. It is also recognised that at the reaction $\mathrm{pH}$ of approximately $12.5, \mathrm{SiO}_{2}$ is predominately present as $\mathrm{H}_{3} \mathrm{SiO}_{4}^{-}$. 
- $\left[\mathrm{SiO}_{2}\right]=0.286 \mathrm{~mol} \mathrm{dm}{ }^{-3}$

The sodium silicate solution was rapidly added to the calcium hydroxide suspension, immediately generating a viscous slurry. Stirring of the resulting calcium silicate precipitate was maintained for about one hour, and then the material was aged by allowing it to stand overnight. Various post-synthesis treatments were applied, depending on the requirements for the final material:

For the preparation of some materials, the ratios of $\mathrm{Ca}(\mathrm{OH})_{2}: \mathrm{HCl}: \mathrm{SiO}_{2}$ were maintained, however, the concentration of the precursor solutions was increased:

- $\left[\mathrm{Ca}\right.$ from $0.312 \mathrm{~mol} \mathrm{dm}^{-3}$ to $0.937 \mathrm{~mol} \mathrm{dm}^{-3}$

- $\left[\mathrm{SiO}_{2}\right]$ from $0.286 \mathrm{~mol} \mathrm{dm}^{-3}$ to $0.858 \mathrm{~mol} \mathrm{dm}^{-3}$

Increasing the concentration of the precursor solutions resulted in a more viscous initial precipitate upon mixing of the two solutions. For this reason, mechanical stirring was provided by a dispersator — an M.T. $420 \mathrm{~W}$ motor (Bologna, Italy) with a T-Verter N2 control unit, operated at 300-400 rpm.

\subsubsection{Water Washed Material}

It has previously been shown ${ }^{20,22}$ that drying the silicate leads to collapse of the pore structure, resulting in a significantly reduced surface area. If such a silicate was desired, the as-made material was recovered by vacuum filtration, and the filter cake washed thoroughly with water. ${ }^{\dagger}$ Then the filter cake was dried in an oven at $110^{\circ} \mathrm{C}$ overnight.

\subsubsection{Filter-cake}

If it was deemed necessary to maintain the pore structure, and hence accessible surface area, the filter cake was stored in a sealed container and not permitted to dry out. The

${ }^{\dagger}$ The ratio of $\mathrm{Ca}: \mathrm{Si}$ in the precursor solutions is $1.09: 1$. Some calcium is removed with the filtrate, ${ }^{22}$ giving a Ca:Si ratio of $0.8: 1$ in the final product. 
solids content of a subsample was determined by taking a known mass of the filter-cake (10 to $20 \mathrm{~g}$ ) and drying it at $110^{\circ} \mathrm{C}$ overnight in an oven. Typically, samples were found to contain between 5 and 15 wt $\%$ solids. ${ }^{\dagger}$

\subsubsection{2-Ethoxyethanol Washed Material}

In some cases, it was necessary to dry the material while maintaining the high surface area. In this case, 2-ethoxyethanol was used as a spacer compound due to its low surface tension compared to $\mathrm{H}_{2} \mathrm{O}$. During the filtration step, the filter cake was first washed well with water to remove any salts $\left(\mathrm{Na}^{+}\right.$and $\mathrm{Cl}^{-}$are spectator ions in the calcium silicate precipitation reaction). After the final water rinse, but before suction through the filter cake was lost, one plug volume of 2-ethoxyethanol was added to the filter funnel. It was allowed to pass through the filter cake, displacing any water present. The filter cake was subsequently dried in a vented oven at $110^{\circ} \mathrm{C}$. Due to the higher boiling point of 2ethoxyethanol, when compared to water, it took longer to dry the 2-ethoxyethanol washed filter-cake than to dry the water washed one. Typically, samples were dried for at least two days prior to use.

\subsubsection{4 pH Adjustment}

The calcium content of the silicate was reduced via the addition of $\mathrm{HCl}$. A calcium silicate slurry $\left(1 \mathrm{dm}^{3}\right)$ prepared from the higher concentration reactants was stirred gently with an overhead stirrer. To this, concentrated $\mathrm{HCl}\left(35 \%, 10.6 \mathrm{~mol} \mathrm{dm}^{-3}\right)$ was added dropwise from a burette, while monitoring the $\mathrm{pH}$ of the slurry. The total volume added was $105 \mathrm{~cm}^{3}$, reducing the $\mathrm{pH}$ from an initial value of 12.9 to 7.4 . The sample was aged overnight, before filtration on a vacuum system, washing well with water. The filter cake was subsequently dried at $110^{\circ} \mathrm{C}$.

\footnotetext{
${ }^{\dagger}$ The efficiency of the filtration system can significantly alter the solids content of the filter-cake.
} 


\subsubsection{Reinforcement}

The structure of the as-made NCS could be reinforced by condensing a layer of $\mathrm{SiO}_{2}$ on top of the pore structure. A stirred, as-made NCS slurry, $\left(50 \mathrm{~cm}^{3}\right.$ of the higher concentration preparation) was diluted with water to an approximate volume of $75 \mathrm{~cm}^{3}$, then sodium silicate $\left(3.21 \mathrm{~g}, 15.3 \mathrm{mmol}\right.$ of $\left.28.7 \mathrm{wt} \% \mathrm{SiO}_{2}\right)$ was added. The reaction was stirred for five minutes to allow the additional silicate to disperse. Over a period of 10 minutes, $2 \mathrm{~mol} \mathrm{dm}{ }^{-3} \mathrm{HCl}\left(5 \mathrm{~cm}^{3}\right)$ was added dropwise from a burette. Stirring was maintained for a one hour to permit time for the $\mathrm{SiO}_{2}$ layer to form. Samples were aged overnight before filtration on a vacuum system. The filter cake was washed with water and subsequently dried at $110^{\circ} \mathrm{C}$.

Reinforcement was also performed with NCS prepared from the lower concentration reactants - in this case, the initial slurry was not diluted before additional sodium silicate was added.

\subsubsection{Synthesis of Magnetic Calcium Silicate Composites}

Two separate methods were employed for the synthesis of magnetic calcium silicate composites: One method provided a magnetite-calcium silicate composite, the other provided a maghemite-calcium silicate composite.

\subsubsection{Magnetite-Calcium Silicate Composite}

For the preparation of composite materials containing magnetite, the NCS was assumed to have an empirical formula of $\mathrm{Ca}_{0.8} \mathrm{SiO}_{2.8} \cdot 2 \mathrm{H}_{2} \mathrm{O}$, which was used to determine the mass ratio of magnetite to silicate. For example, to synthesise a material containing 25 wt $\% \mathrm{Fe}_{3} \mathrm{O}_{4}$, magnetite $(3.36 \mathrm{~g}, 14.5 \mathrm{mmol})$ was added to a sodium silicate solution as detailed in the general procedure. The mixture was blended with a stirring rod for five minutes in order to ensure that the $\mathrm{Fe}_{3} \mathrm{O}_{4}$ was thoroughly dispersed. At this point, the magnetite-containing sodium silicate solution was added to the calcium hydroxide 
suspension as per the general synthetic procedure. Once the material had aged overnight, post-synthesis treatments such as reinforcement or 2-ethoxyethanol washing could be carried out. If large quantities of the material were desired (a total reaction volume greater than $500 \mathrm{~cm}^{3}$ ), the magnetite was dispersed in the sodium silicate solution via the use of a mechanical overhead stirrer equipped with a plastic mixing blade. This method proved suitable for the synthesis of composites ranging from 1-25 wt $\% \mathrm{Fe}_{3} \mathrm{O}_{4}$, without significantly degrading the accessible surface area of the silicate. Attempts were not made to synthesise composites containing a greater percentage of $\mathrm{Fe}_{3} \mathrm{O}_{4}$.

\subsubsection{Maghemite-Calcium Silicate Composite}

Magnetite nanoparticles having a nominal diameter of $15 \mathrm{~nm}$ were prepared according to the method of Berger, ${ }^{45}$ itself based on the co-precipitation method of Massart. ${ }^{46}$ The iron salts $\mathrm{FeCl}_{2} \cdot 4 \mathrm{H}_{2} \mathrm{O}, 10.53 \mathrm{~g}(0.053 \mathrm{~mol})$, and $\mathrm{FeCl}_{3} \cdot 6 \mathrm{H}_{2} \mathrm{O}, 28.65 \mathrm{~g}$ (0.106 mol), were dissolved in $132.5 \mathrm{~cm}^{3}$ of $2 \mathrm{~mol} \mathrm{dm}{ }^{-3} \mathrm{HCl}$, providing a solution $0.40 \mathrm{~mol} \mathrm{dm}^{-3}$ in $\mathrm{Fe}^{2+}$ and $0.80 \mathrm{~mol} \mathrm{dm}{ }^{-3}$ in $\mathrm{Fe}^{3+}$. Ammonia solution $\left(1 \mathrm{~mol} \mathrm{dm}{ }^{-3}, 1325 \mathrm{~cm}^{3}\right)$ was added dropwise to the rapidly stirred iron solution over the course of six hours. The addition of $\mathrm{NH}_{3}$ caused the formation of a fine black $\mathrm{Fe}_{3} \mathrm{O}_{4}$ suspension after first progressing through an iron hydroxide floc. The reaction mixture was stirred for a further 30 minutes after completion of the $\mathrm{NH}_{3}$ addition. The magnetite was precipitated by placing a rare-earth magnet under the reaction flask, and the supernatant decanted off. The magnetite was resuspended in $500 \mathrm{~cm}^{3}$ of water, and the precipitation step repeated until the magnetite had been rinsed four times, at which point the supernatant had a pH value of approximately 7 . The magnetite was finally suspended in $500 \mathrm{~cm}^{3}$ water and the solids content determined by drying a subsample on a rotary evaporator.

The composite materials were synthesised as described in the previous section, wherein the magnetite suspension was dispersed in a sodium silicate solution for 10 minutes with mechanical stirring prior to its addition to a calcium hydroxide slurry. The synthesised composites were subsequently treated with 2-ethoxyethanol and oven dried at $110^{\circ} \mathrm{C}$ overnight, resulting in the magnetite, $\mathrm{Fe}_{3} \mathrm{O}_{4}$, being oxidised to the maghemite phase, 
$\gamma-\mathrm{Fe}_{2} \mathrm{O}_{3}$. This was evidenced by XRD and a change in the colour of the composites from grey to orange. The final composite still exhibited magnetic characteristics.

\subsubsection{Synthesis of Calcium Silicate-Polyaniline Composites}

\subsubsection{Synthesis of Polyaniline}

Polyaniline (PANI), in its emeraldine salt form was prepared as follows:

Aniline $\left(0.455 \mathrm{~cm}^{3}, 5 \mathrm{mmol}\right)$ and sodium dodecylbenzene sulfonate (SDBS), $0.17 \mathrm{~g}$ (0.5 mmol), were dissolved in $100 \mathrm{~cm}^{3}$ of $0.5 \mathrm{~mol} \mathrm{dm}{ }^{-3} \mathrm{H}_{2} \mathrm{SO}_{4}$ under vigorous stirring in a round-bottom flask, providing a solution $0.05 \mathrm{~mol} \mathrm{dm}^{-3}$ in anilinium and $0.005 \mathrm{~mol} \mathrm{dm}^{-3}$ in SDBS. This solution was cooled on an ice bath to a temperature of approximately $0^{\circ} \mathrm{C}$. Separately, ammonium peroxydisulfate, $\left(\mathrm{NH}_{4}\right)_{2} \mathrm{~S}_{2} \mathrm{O}_{8}(1.71 \mathrm{~g}$, $7.5 \mathrm{mmol}$ ), was dissolved in $100 \mathrm{~cm}^{3}$ of water, to provide a $0.075 \mathrm{~mol} \mathrm{dm}^{-3}$ solution. The ammonium peroxydisulfate solution was added dropwise to the stirred, cool anilinium solution over a period of 60 minutes. During this time, the solution was observed to develop a blue-green tint. Once the addition of the oxidant was complete, the ice bath was removed, and the reaction mixture permitted to warm to room temperature. Stirring was continued for a further 18 hours, by which time dark black-green emeraldine salt had formed. Then the PANI suspension was transferred to dialysis tube with a molecular mass cutoff at $14 \mathrm{kDa}$ (Membracell MD44-14) and dialysed against $\mathrm{H}_{2} \mathrm{O}$ in a $5 \mathrm{dm}^{3}$ beaker for a period of five days, exchanging the dialyate twice daily. Once dialysis was complete, the PANI suspension was removed from the membrane and water was removed by rotary evaporation. The mass of black-green PANI powder recovered was $0.65 \mathrm{~g}$, equivalent to a yield of $93.5 \%$ assuming that the polyaniline was completely in the emeraldine sulfate form, $\left(\mathrm{C}_{6} \mathrm{H}_{5} \mathrm{~N}^{+\cdot} \cdot \frac{1}{2} \mathrm{SO}_{4}^{2-}\right)_{\mathrm{n}}\left(M_{r}=139.2 \mathrm{~g} \mathrm{~mol}^{-1}\right)$. 


\subsubsection{Synthesis of the Composite}

PANI was incorporated into NCS via sorption of PANI from solution. Typically, composite materials containing less than $10 \mathrm{wt} \%$ PANI were prepared. The following relates to the synthesis of a composite containing $5 \mathrm{wt} \%$ PANI. To incorporate PANI into the NCS, the polymer $\left(26 \mathrm{mg}, 0.19 \mathrm{mmol}\right.$ based on the $\mathrm{C}_{6} \mathrm{H}_{5} \mathrm{~N}^{+\cdot} \cdot \frac{1}{2} \mathrm{SO}_{4}^{2-}$ repeat unit) was dissolved in $10 \mathrm{~cm}^{3} 1$-methylpyrrolidine (NMP) in a $50 \mathrm{~cm}^{3}$ flask, providing a dark blue solution. In order to ensure that the PANI maintained its deprotonated state, $\mathrm{NaOH}(0.40 \mathrm{~g}, 10 \mathrm{mmol})$ was added to the flask. NCS $(0.50 \mathrm{~g})$ was then added to the stirred PANI solution. The reaction flask was stoppered to prevent evaporation of NMP and stirring was maintained for 18-24 hours. At this point, the PANI-NCS composite was slowly filtered off, leaving the largely undissolved $\mathrm{NaOH}$ pellets in the reaction flask. The $\mathrm{NaOH}$ was dissolved in $50 \mathrm{~cm}^{3} \mathrm{H}_{2} \mathrm{O}$, giving a solution of approximately $0.2 \mathrm{~mol} \mathrm{dm}^{-3}$. The filter cake was washed with this solution, followed by three additional rinsings, each $50 \mathrm{~cm}^{3} \mathrm{H}_{2} \mathrm{O}$. A final washing was performed with ethanol. While the majority of the PANI remained bound to the NCS, a small amount was observed in the filtrate, which was a pale blue colour. The filter cake was dried in vacuo in a desiccator above silica gel in order to avoid possible degradation of PANI at elevated temperatures. Once dry, the solids possessed a blue colour throughout. If composites were desired with a greater percentage of PANI, more PANI was initially dissolved in the NMP. The volume of solvent used was determined by the mass of NCS desired - more PANI could easily be dissolved in the solvent, but $0.5 \mathrm{~g} \mathrm{NCS}$ in $10 \mathrm{~cm}^{3}$ NMP provided a thick slurry. In cases where the material was washed with 2-ethoxyethanol during filtration, it was observed that additional PANI was washed out of the filter-cake.

The procedure was repeated using either the reinforced analogue of NCS, or omitting the ethanol washing step. Ethanol was chosen for the final washing in preference to 2-ethoxyethanol as the latter had a tendency to leach extra PANI out of the composite material, most likely due to the 2-ethoxyethanol and the PANI binding to the same sites on the NCS surface. ${ }^{47}$

The materials were characterised via TGA and spectroscopic techniques. Composite 
materials were also prepared containing ${ }^{15} \mathrm{~N}-\mathrm{PANI}$ in a similar manner to that described above, using $>98$ atom $\%{ }^{15} \mathrm{~N}$ aniline. It was initially thought that ${ }^{15} \mathrm{~N}-\mathrm{NMR}$ could be undertaken with the composites, but the obtained spectra were poorly resolved. Instead, the ${ }^{15} \mathrm{~N}$-PANI composites were characterised by XPS spectroscopy.

\subsection{Sorption Studies of Copper}

\subsubsection{Kinetic Studies}

Copper-containing solutions with $\mathrm{Cu}^{2+}$ concentration ranging from $5000 \mathrm{mg} \mathrm{dm}^{-3}$ to $100 \mathrm{mg} \mathrm{Cu}^{2+} \mathrm{dm}^{-3 \dagger}$ were prepared from either chloride or nitrate salts, $\mathrm{CuCl}_{2} \cdot 2 \mathrm{H}_{2} \mathrm{O}$ or $\mathrm{Cu}\left(\mathrm{NO}_{3}\right)_{2} \cdot 3 \mathrm{H}_{2} \mathrm{O}$. A known volume of each copper solution $\left(1 \mathrm{dm}^{3}\right)$, was transferred to a separate Erlenmeyer flask, and magnetically stirred such that a mild vortex was present. To each of these copper solutions a known mass of 2-ethoxyethanol washed, dry NCS (approximately $1 \mathrm{~g}$ ) was added. Samples of $12 \mathrm{~cm}^{3}$ were withdrawn from these flasks at intervals over the next 24 hours. Typically, 8 samples were withdrawn in the first hour. Immediately upon withdrawing each sample, it was passed through a $0.45 \mu \mathrm{m}$ membrane, discarding the first $2-4 \mathrm{~cm}^{3}$ of the filtrate. The remainder $\left(8-10 \mathrm{~cm}^{3}\right)$ of each filtrate was retained and stored separately in plastic vials until the sorption experiment had concluded. The filtrates were subsequently analysed to determine $\mathrm{pH}$, and the concentration of both copper and calcium.

Once the sorption experiment had concluded, the spent NCS was recovered via filtration, rinsing with $\mathrm{H}_{2} \mathrm{O}$, followed by 2-ethoxyethanol. The recovered NCS was dried and analysed by X-ray powder diffraction (XRD) and electron microscopy (SEM and EDS).

This procedure was repeated for the following derivatives of NCS:

- $\mathrm{pH}$ adjusted NCS

\footnotetext{
$\dagger$ Although the solutions were prepared on a mass per volume basis $\left(\mathrm{mg} \mathrm{dm}^{-3}\right)$, discussions in the text will refer to the concentration on a molar basis. Thus, for $\mathrm{Cu}^{2+} 5000 \mathrm{mg} \mathrm{dm}^{-3}$ is $79 \mathrm{~mol} \mathrm{dm}^{-3} ; 500 \mathrm{mg} \mathrm{dm}^{-3}$ is $7.9 \mathrm{~mol} \mathrm{dm}^{-3} ; 100 \mathrm{mg} \mathrm{dm}^{-3}$ is $1.6 \mathrm{~mol} \mathrm{dm}^{-3}$.
} 
- NCS filter-cake

- Magnetic NCS composites

\subsubsection{Isotherm Measurements}

Copper-containing solutions $\left(50 \mathrm{~cm}^{3}\right)$ were prepared from $\mathrm{CuCl}_{2} \cdot 2 \mathrm{H}_{2} \mathrm{O}$, having $\mathrm{Cu}$ concentrations of $0,1.0,2.0,3.0,4.0,5.0,6.0,7.0,8.0,9.0,10.0 \mathrm{mmol} \mathrm{dm}^{-3}$. NCS, in the form of a filter cake, with a dry mass equivalent to $0.025 \mathrm{~g}$, was added to each of a series of $50 \mathrm{~cm}^{3}$ glass centrifuge tubes. The copper solutions were added to these tubes, whereupon the tubes were sealed and shaken on a flask shaker (Labnet Orbit 300) for 24 hours. At this point, the mixtures were centrifuged at approximately $1000 \mathrm{rpm}$ for 15 minutes. Subsamples of the supernatant were analysed to determine $\mathrm{pH}, \mathrm{Ca}$ and $\mathrm{Cu}$ concentrations. From these data, isotherm curves were constructed.

The experiments were repeated with a magnetite-containing NCS (25 wt $\% \mathrm{Fe}_{3} \mathrm{O}_{4}$ ).

\subsection{Sorption Studies of Phosphate}

\subsubsection{Isotherm Studies}

Into a series of dry $100 \mathrm{~cm}^{3}$ flasks samples of calcium silicate-magnetite composites in the form of filter cakes were added. Phosphate solutions were prepared from $\mathrm{NaH}_{2} \mathrm{PO}_{4} \cdot \mathrm{H}_{2} \mathrm{O}$ in the concentration range from 0.01 to $10 \mathrm{mmol} \mathrm{dm}{ }^{-3}$. These solutions $\left(50 \mathrm{~cm}^{3}\right)$ were added to the NCS-containing flasks. Then, the flasks were stirred on a multiposition magnetic stirrer (IKA-Werke EOA9) for 24 hours at room temperature (23$25{ }^{\circ} \mathrm{C}$ ). Once this time period had elapsed, the stirring was halted and the silicate was permitted to settle for about 10 minutes. A portion of the supernatant $\left(30-40 \mathrm{~cm}^{3}\right)$ was withdrawn via syringe and passed through a cellulose membrane having a pore size not exceeding $0.45 \mu \mathrm{m}$. The $\mathrm{pH}$ value of each filtrate was determined, as was phosphate concentration. If the samples were not immediately analysed, they were stored 
in glass vials at $4{ }^{\circ} \mathrm{C}$. The quantity of phosphate sorbed by the silicate was calculated by difference. Isotherms were calculated for samples with sorbent loadings at $0.5,1.0$ and $2.0 \mathrm{~g} \mathrm{NCS}$ composite $\mathrm{dm}^{-3}$ (dry basis). Isotherm measurements were primarily conducted with a calcium silicate containing $25 \mathrm{wt} \%$ (dry basis) magnetite, however the sorption capacity was also determined for composites covering the range of magnetite content - from 0 to 25 wt $\%$, with a control sample of $100 \%$ magnetite likewise being analysed.

\subsubsection{Kinetic Studies}

A $2 \mathrm{dm}^{3}$ flask was loaded with $1 \mathrm{dm}^{3}$ of a $1.1 \mathrm{mmol} \mathrm{dm}^{-3}$ phosphate solution (prepared from $\mathrm{NaH}_{2} \mathrm{PO}_{4} \cdot \mathrm{H}_{2} \mathrm{O}$ ). In a separate flask, a $\mathrm{Fe}_{3} \mathrm{O}_{4}-\mathrm{NCS}$ filter cake containing 25 wt $\%$ $\mathrm{Fe}_{3} \mathrm{O}_{4}$ (1.1 g dry equivalent) was dispersed in $100 \mathrm{~cm}^{3}$ water under vigorous stirring for $30 \mathrm{~min}$. The silicate suspension was added quickly to the stirred $(300 \mathrm{rpm}$, moderate vortex present) phosphate solution. Over the course of $60 \mathrm{~min}$, nine samples, each of $25 \mathrm{~cm}^{3}$ volume, were withdrawn using a graduated syringe pipette. The samples were promptly filtered through a $0.45 \mu \mathrm{m}$ membrane into glass vials. The filtrates were subsequently analysed to determine their phosphate content and $\mathrm{pH}$ values. The $\mathrm{pH}$ value was also monitored in situ during the initial stages of the reaction.

\subsubsection{Continuous Uptake Studies}

Column sorption experiments were run in up-flow mode as to approximate a fluidised bed sorption. The system schematic is shown in Figure 2.1. Glass columns of two different sizes were employed - either $50 \mathrm{~cm}^{3}$ or $600 \mathrm{~cm}^{3}$. The column was connected to a peristaltic pump (PLP 33, Labor-Technik Dusseldorf) with silicone tubing, with an influent connection made at the bottom of the column while an effluent connection was provided via a side arm near the top of the column. The influent reservoir was a $2 \mathrm{dm}^{3}$ flask. The smaller column was cylindrical, having parallel sides, whereas the larger column was conical, having been constructed from a $1 \mathrm{dm}^{3}$ pear-shaped separating funnel 


$$
\mathrm{H}_{2} \mathrm{PO}_{4}^{-} \text {Stock }
$$

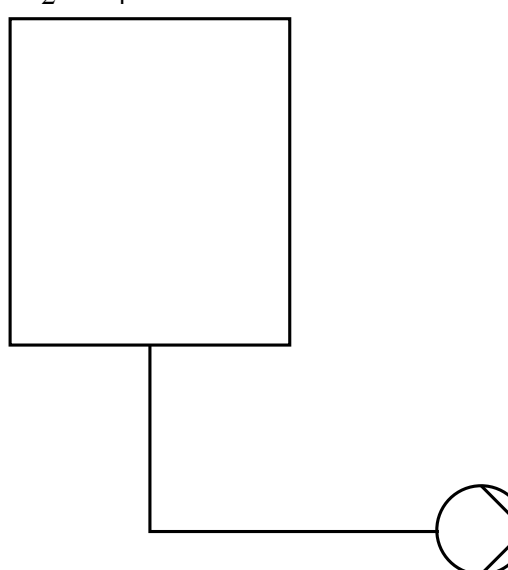

(a)

(b)

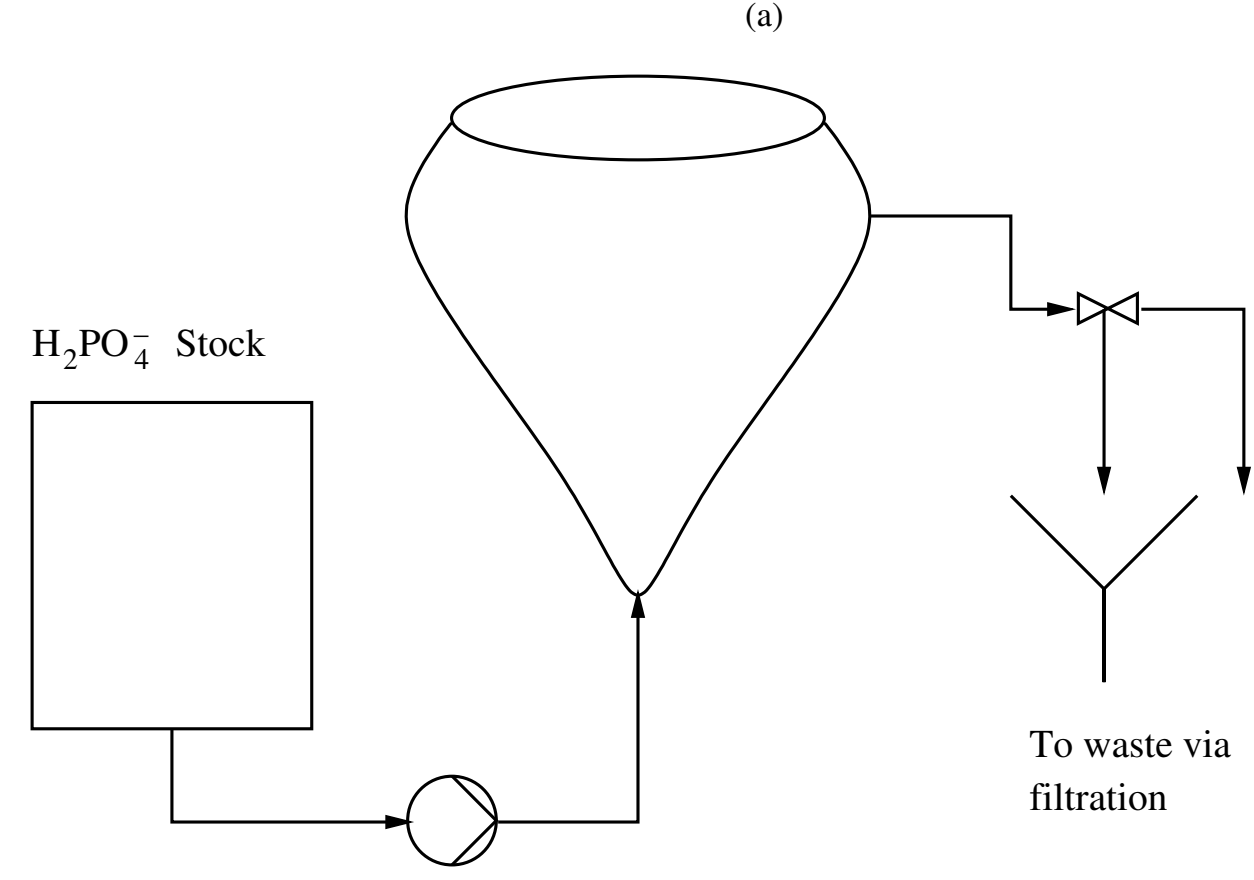

collection

To waste via

\section{collection}

filtration

Frit
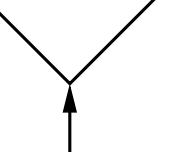

To sample collection

Figure 2.1 Schematic of set-up for column sorption experiments: (a) $50 \mathrm{~cm}^{3}$ column; (b) $600 \mathrm{~cm}^{3}$ column. 
by removing its upper half. The effluent was usually passed through a piece of filter paper to capture any silicate leaving the column, however a valve was provided in the effluent tubing to enable subsamples to be taken for analysis. Typically, $20-25 \mathrm{~cm}^{3}$ samples were collected in a syringe and passed through a $0.45 \mu \mathrm{m}$ membrane before being stored in glass vials. The exact volume taken depended on the pump speed as the sampling was undertaken for a known time period. For the smaller column, the pump was set to deliver 15 column volumes per hour and samples were taken after every two column volumes (two minutes of sampling in every eight minute cycle). For the larger column, the pump was set to maximum speed and delivered four column volumes per hour. A 30 second sampling period was allowed every 10 minutes. Columns were loaded with $\mathrm{Fe}_{3} \mathrm{O}_{4}-\mathrm{NCS}$ composites containing $25 \mathrm{wt} \% \mathrm{Fe}_{3} \mathrm{O}_{4}$ (dry basis). The silicate was applied in the form of filter cake at the following loading:

- For the $50 \mathrm{~cm}^{3}$ column: $0.25 \mathrm{~g}$ dry material per litre of influent $(10 \mathrm{~g}$ per litre of column volume)

- For the $600 \mathrm{~cm}^{3}$ column: $1.00 \mathrm{~g}$ dry material per litre of influent $(3.33 \mathrm{~g}$ per litre of column volume)

Typically, the filter cake was dispersed in water and the column filled with water before the phosphate-containing influent was introduced. However, experiments were conducted in which the filter cake was not predispersed, but instead, was dispersed via manual stirring with a glass rod as the column filled with influent.

\subsection{Sorption Studies of Rhenium}

\subsubsection{Sorption by Polyaniline Films}

Aniline, $0.230 \mathrm{~cm}^{3}(2.5 \mathrm{mmol})$, was dissolved in $50 \mathrm{~cm}^{3}$ of $0.5 \mathrm{~mol} \mathrm{dm}^{-3}$ sulfuric acid, affording a solution of $0.05 \mathrm{~mol} \mathrm{dm}^{-3}$ anilinium. Separately, the oxidant $\left(\mathrm{NH}_{4}\right)_{2} \mathrm{~S}_{2} \mathrm{O}_{8}$ $(0.86 \mathrm{~g}, 4.0 \mathrm{mmol})$, was dissolved in $50 \mathrm{~cm}^{3}$ of $0.5 \mathrm{~mol} \mathrm{dm}^{-3}$ sulfuric acid, providing 
a $0.075 \mathrm{~mol} \mathrm{dm}{ }^{-3}$ solution of the oxidant. Both solutions were cooled to approximately

$0^{\circ} \mathrm{C}$ on an ice bath before the anilinium solution was added to the oxidant under vigorous stirring. Thirty seconds were allowed for the solutions to mix, then subsamples of this solution $\left(4 \mathrm{~cm}^{3}\right)$ were added to each of a series of $5 \mathrm{~cm}^{3}$ disposable polycarbonate cuvettes. The cuvettes were permitted to stand overnight by which time dark green polyaniline had formed. The cuvettes were emptied and rinsed with sequential washings of water, ethanol, and water again to remove excess polyaniline. Upon drying, a thin film of polyaniline remained on the inside of each cuvette.

The UV/vis spectra of these polyaniline films were recorded, in solutions of either: $0.1 \mathrm{~mol} \mathrm{dm}^{-3} \mathrm{HCl}, 0.1 \mathrm{~mol} \mathrm{dm}{ }^{-3} \mathrm{NaOH}, 0.1 \mathrm{~mol} \mathrm{dm}^{-3}\left(\mathrm{NH}_{4}\right)_{2} \mathrm{~S}_{2} \mathrm{O}_{8}$ or $0.1 \mathrm{~mol} \mathrm{dm}^{-3}$ $\mathrm{N}_{2} \mathrm{H}_{4} \cdot \mathrm{H}_{2} \mathrm{O}$ to access the emeraldine salt, emeraldine base, pernigraniline salt and leucoemeraldine base states of the polymer respectively.

A solution, containing $1000 \mathrm{mg} \mathrm{Re} \mathrm{dm}{ }^{-3}\left(5.4 \mathrm{mmol} \mathrm{dm}{ }^{-3}\right.$, as $\left.\mathrm{KReO}_{4}\right)$ in $0.1 \mathrm{~mol} \mathrm{dm}^{-3}$ $\mathrm{HCl}$, was added to cuvettes coated with emeraldine salt or leucoemeraldine base, having first rinsed the cuvette with water to remove any excess reagents. Any changes in the oxidation state of the polymer due to its interaction with the perrhenate was followed spectrophotometrically.

\subsubsection{Sorption by PANI-NCS Composites}

Fifty milligrams samples of the PANI-NCS composite (described in Section 2.2.3.2, containing $10 \mathrm{wt} \%$ PANI) were added to each of a series of $50 \mathrm{~cm}^{3}$ glass centrifuge tubes. Mostly, composites containing ${ }^{14} \mathrm{~N}-\mathrm{PANI}$ were used, but an additional sorption measurement was made with a ${ }^{15} \mathrm{~N}-\mathrm{PANI}-\mathrm{NCS}$ composite and the most concentrated

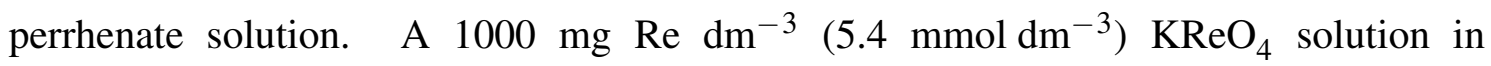
$0.01 \mathrm{~mol} \mathrm{dm}^{-3} \mathrm{HCl}$ was prepared by dissolving $0.3884 \mathrm{~g} \mathrm{KReO}_{4}(1.343 \mathrm{mmol})$ in $250 \mathrm{~cm}^{3}$ of $0.01 \mathrm{~mol} \mathrm{dm}^{-3} \mathrm{HCl}$. Serial dilutions of the rhenium solution were made

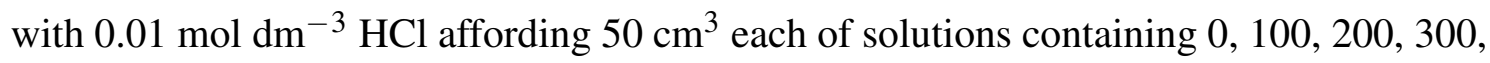
400, 500, 600 and $1000 \mathrm{mg} \mathrm{Re} \mathrm{dm}^{-3}$. These solutions were added to the tubes containing the PANI-NCS composite. The tubes were shaken vigorously for a few minutes, then the 
perrhenate solution was left in static contact with the PANI-NCS composite for 24 hours. Following this, subsamples of the supernatant were removed and analysed to determine rhenium and calcium concentrations, and $\mathrm{pH}$ values. The PANI-NCS composites were recovered by filtration and rinsed thoroughly, initially with water, then ethanol. XPS analysis was undertaken on the ${ }^{15} \mathrm{~N}$-PANI sample. 


\section{Chapter 3}

\section{Copper Sorption}

\subsection{Introduction}

\subsubsection{Heavy Metals}

There is no chemically precise definition of the term Heavy Metal. In a review in 2002, Duffus $^{48}$ demonstrated that the term was used in a wide range of contexts. In some cases the term was used to classify metals possessing a high density; ${ }^{\dagger}$ sometimes the definition was based on atomic mass or atomic number; in other cases the definition simply referred to metals or metalloids commonly used in industrial processes which exhibited a high degree of toxicity. Because of this lack of consistency in the usage of this term, Heavy Metal is not defined in the IUPAC Compendium of Chemical Terminology. ${ }^{49}$ For this reason, the term has been avoided in this discussion. Copper was chosen as a target sorbate, as it shares similar chemical characteristics to many of the (often toxic) $d$-block and late $p$-block metals. As such, results obtained from copper sorption studies should be transferable to, for example, lead or mercury.

\subsubsection{Chemistry of Copper}

Copper belongs to the $d$-block metals and has common oxidation states of $0,+1$ and +2 . As $\mathrm{Cu}^{2+}$, it acts as a moderately soft Lewis acid and exhibits a strong affinity towards many soft Lewis bases such as sulfur-based ligands. While it is not a truly hard Lewis acid such as the $s$-block cations, $\mathrm{Cu}^{2+}$ also exhibits a strong affinity for moderately hard Lewis bases such as $\mathrm{NH}_{3}$, with a lesser affinity for the harder, oxygen based, Lewis bases. As a result of this, $\mathrm{CuS}$ and $\mathrm{Cu}(\mathrm{OH})_{2}$ are insoluble, and $\left[\mathrm{Cu}\left(\mathrm{NH}_{3}\right)_{4}\right]^{2+}$ exists as a stable complex. The solubility products of some common copper-based precipitates and stability

${ }^{\dagger}$ The minimum value of high density also varied - from $3.5 \mathrm{~g} \mathrm{~cm}^{-3}$ to $7 \mathrm{~g} \mathrm{~cm}^{-3}$. 
constants of some copper-based complexes are given in Table 3.1. Similar characteristics are shared by many of the $d$-block elements and some of the heavier $p$-block metals such as lead.

Table 3.1 Solubility products and stability constants of some metal-containing species (after Alward and Findlay, ${ }^{50}$ unless otherwise referenced).

\begin{tabular}{lrr}
\hline Species & $\mathbf{p} K_{s p}$ & $\mathbf{p} K$ \\
\hline $\mathrm{Cu}(\mathrm{OH})_{2}$ & 19.3 & \\
$\mathrm{CuS}$ & 36 & \\
$\mathrm{Cu}_{2} \mathrm{Cl}(\mathrm{OH})_{3}{ }^{51}$ & 17.27 & \\
$\mathrm{Cu}_{2}\left(\mathrm{NO}_{3}\right)(\mathrm{OH})_{3}{ }^{51}$ & 16.37 & \\
${\left[\mathrm{Cu}\left(\mathrm{NH}_{3}\right)_{4}\right]^{2+}}$ & $-11.75^{a}$ \\
{$[\mathrm{Cu}(\mathrm{EDTA})]^{2-}$} & & -18.78 \\
$\mathrm{~Pb}(\mathrm{OH})_{2}$ & 16 & \\
{$\left[\mathrm{~Pb}(\mathrm{OH})_{4}\right]^{2-52}$} & & -15.2 \\
$\mathrm{PbS}$ & 27.5 & \\
\hline
\end{tabular}

${ }^{a}$ This value is given for the equilibrium $\mathrm{Cu}^{2+}+4 \mathrm{NH}_{3} \rightleftharpoons\left[\mathrm{Cu}\left(\mathrm{NH}_{3}\right)_{4}\right]^{2+}$, which is identical in magnitude to the $\beta_{4}$ value (for the equilibrium $\left[\mathrm{Cu}\left(\mathrm{NH}_{3}\right)_{3}\right]^{2+}+$ $\left.\mathrm{NH}_{3} \rightleftharpoons\left[\mathrm{Cu}\left(\mathrm{NH}_{3}\right)_{4}\right]^{2+}\right) .50$

Because of the affinity of copper (or similar species) towards sulfur- and nitrogencontaining ligands, bio-accumulation can occur, where the metal binds strongly to sulfur or nitrogen functional groups in proteins. Bio-accumulation of metals can also occur with other classes of molecules such as polysaccharides. ${ }^{53}$ Once bound, the metal is often very difficult to displace. Unless the organism has developed specific methods for its removal, ${ }^{54}$ the accumulation of copper can lead to chronic toxicity as a result of overexposure. By coordinating to proteins, the metal ion can alter the protein's conformation and retard its biological function. In the case of copper, all plants and animals require it as an essential nutrient. A typical adult human requires a few micrograms of copper per kilogram of body mass. ${ }^{55}$ Overexposure can still occur: for $\mathrm{CuSO}_{4} \cdot 5 \mathrm{H}_{2} \mathrm{O}$ the oral $\mathrm{LD}_{50}$ value for acute exposure in rats has been reported as $300 \mathrm{mg} \mathrm{kg}^{-1} .{ }^{56}$ Copper compounds are often employed for antibacterial or antifungal purposes. ${ }^{57}$ Other chemical species, not required as micronutrients, such as lead or mercury compounds act in a similar way. As the organism does not require these metal species, specific mechanisms for excretion are often underdeveloped. Instead the organism may rely on renal excretion, which strongly depends on the solubility and 
hydrophilicity of the species involved. ${ }^{54}$

As many of the toxic metal species exhibit similar chemical characterstics to copper, $\mathrm{Cu}^{2+}$ was deemed to be a representative metal ion for the purposes of testing the sorption characteristics of NCS.

\subsubsection{Copper Sorption Techniques}

The aims of many techniques applied to the sorption of copper are recycling or water treatment, for example the recovery of copper from electroplating wastewater. For example, a typical electroplating wastewater stream contains metal ions at concentrations of approximately $1 \mathrm{mmol} \mathrm{dm}^{-3}$ either from a copper-coating bath ${ }^{58}$ or as a minor component of chromium or nickel electroplating baths. ${ }^{59}$ Methods used for the recovery of copper (or other metals) often involve ion exchange resins, ${ }^{58,60-62}$ chelating resins, ${ }^{63}$ ionic liquids, ${ }^{64}$ electrochemical techniques ${ }^{65-71}$ or precipitation of the metal species by sulfides. ${ }^{72}$ In practical applications many of these techniques are applied in sequence. For example, after initial precipitation of the metal ions, the precipitate is dissolved in water, and then ion exchange is used to concentrate the metal species followed by electrochemical recovery. ${ }^{58}$

\subsubsection{Electrochemical Techniques}

Removal of $\mathrm{Cu}^{2+}$ from aqueous solution can be accomplished by electrochemical means as $\mathrm{Cu}^{2+}$ has a high reduction potential with respect to many other metal species. The method of cementation is often employed whereby, a wastewater such as electroplating waste ${ }^{70}$ or pot ale from whisky stills ${ }^{65}$ with a high copper concentration $\left(\left[\mathrm{Cu}^{2+}\right] \geq 1 \mathrm{mmol} \mathrm{dm}^{-3}\right)$ is allowed to react with an elemental metal. Aluminium, ${ }^{65}$ iron, ${ }^{68,69,73}$ or zinc ${ }^{73}$ are often employed for this purpose. Upon contact with the metal surface, $\mathrm{Cu}^{2+}$ is reduced to $\mathrm{Cu}^{0}$, in turn oxidising the sacrificial metal:

$$
\mathrm{Cu}^{2+}+\mathrm{M}(\mathrm{s}) \longrightarrow \mathrm{Cu}(\mathrm{s})+\mathrm{M}^{2+}
$$


Near quantitative uptake of $\mathrm{Cu}^{2+}$ may be achieved according to Equation (3.1). For example, Hor and Mohamed ${ }^{68}$ have reported $100 \%$ of copper being sorbed from a $1.6 \mathrm{mmol} \mathrm{dm}{ }^{-3} \mathrm{Cu}^{2+}$ solution in contact with $\mathrm{Fe}(\mathrm{s})$ for a few minutes in a flow-through reactor. While the technique works well for the recovery (and potential recycling) of copper, disposal of the effluent (now containing the ionic sacrificial metal) may be problematic in many cases. Typically with iron or aluminium however, exposing the effluent to soil is thought to quickly convert the $\mathrm{Al}^{3+}$ and $\mathrm{Fe}^{2+}$ to their harmless oxides, provided that the effluent or soil are not overly acidic. ${ }^{65}$

\subsubsection{Sorption by Biomass}

Because of the large affinity that copper exhibits for nitrogen ligands, sorbents based on biological materials have often been used for the recovery of copper. In a recent review of biomas-derived sorbent materials used for the uptake of metal ions, Ahluwalia and Goyal $^{74}$ reported studies of copper sorption from biosorbents derived from 12 species of fungi, 3 species of bacteria, 11 species of algae, and 12 general plant-derived biomasses. Reported sorption capacities for copper varied widely: from $6 \times 10^{-3}$ to $0.63 \mathrm{mmol} \mathrm{g}^{-1}$ for fungal derived biomass; 0.60 to $1.2 \mathrm{mmol} \mathrm{g}^{-1}$ for algal derived biomass; from 0.14 to $6.0 \mathrm{mmol} \mathrm{g}^{-1}$ for bacterial derived biomass; and $9.71 \times 10^{-2}$ to $0.38 \mathrm{mmol} \mathrm{g}^{-1}$ for plant derived biomass.

Many biomass-derived sorbent materials originate as waste products from other processes. Crab shell, for example, can often be obtained at little or no cost from the fishing industry. This means that biomass sorbents often have a significant cost advantage over other potential sorbents such as ion-exchange resins. In some cases, biosorbents can exhibit better characteristics than commercial ion exchange resins. When comparing a crab shell biosorbent to a commercial ion exchange resin (Dowex ${ }^{\circledR}$ 50WX4) Cochrane et al. ${ }^{75}$ observed that although the resin sorbed $\mathrm{Cu}^{2+}$ at a faster rate, the crab shell biosorbent possessed a larger sorption capacity. The ion exchange resin $\left(0.5 \mathrm{~g} \mathrm{dm}^{-3}\right)$ removed

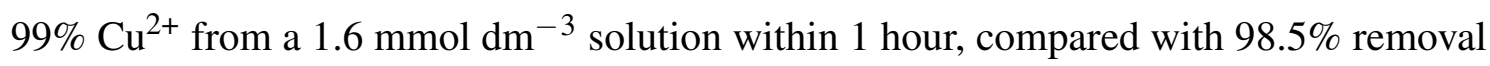
after 12 hours for the crab shell. The maximum sorption capacities were reported as 
$1.12 \mathrm{mmol} \mathrm{g}^{-1}$ and $1.25 \mathrm{mmol} \mathrm{g}^{-1}$ for the resin and crab shell respectively.

\subsubsection{Mechanism of Sorption}

While many proteins will coordinate strongly to metal ions, very often they will only bind the metal at specific sites within the protein. For this reason, the sorption capacity of metal ions by proteins in vivo is low. ${ }^{76}$ Muraleedharan and Venkobachar ${ }^{77}$ have investigated, by EPR spectroscopy, the mechanism of $\mathrm{Cu}^{2+}$ sorption by biomass derived from the fungus Ganoderma lucidium. They concluded that proteins were not responsible for copper sorption; removal of proteins from the biomass resulted in a slight increase in copper sorption efficiency due to the proteins obstructing potential sorption sites. Another nitrogen-containing compound often thought to be responsible for copper sorption, chitin, (Figure 3.1) was only found to contribute 5\% of the observed copper uptake. Other researchers ${ }^{78}$ have demonstrated similar results with other fungal species, showing that interactions between $\mathrm{Cu}^{2+}$ and oxygen-based ligands such as polysaccharides ${ }^{53}$ are the major contributor to biomass-derived sorption. In a review of biomass sorbents by Gardea-Torresdey, ${ }^{79}$ it was noted that binding often occurs between metal ions and carboxyl moieties, although binding to sulfur or nitrogen groups is also known. Carboxylate groups within the biomass can also act in an ion-exchange capacity due to the equilibrium:

$$
\mathrm{R}-\mathrm{COOH} \rightleftharpoons \mathrm{R}-\mathrm{COO}^{-}+\mathrm{H}^{+}
$$

It has been suggested in some cases that precipitation of $\mathrm{Cu}(\mathrm{OH})_{2}$ can occur on the surface of the biomass. ${ }^{80}$ As a result of sorption of $\mathrm{Cu}^{2+}$ ions by co-ordination to carboxylate groups (or ion exchange) the concentration of the metal ion near the surface of the biomass increases (through the formation of an electrical double layer). This can lead to the copper concentration exceeding the solubility limit for $\mathrm{Cu}(\mathrm{OH})_{2}$ resulting in precipitation of the hydroxide. It has been suggested that for a sorption system having a total $\mathrm{Cu}^{2+}$ concentration of $0.1 \mathrm{mmol} \mathrm{dm}^{-3}$, the solubility limit of $\mathrm{Cu}(\mathrm{OH})_{2}$ will be exceeded in the double layer at a $\mathrm{pH}$ in excess of 5.0. By comparison, a bulk solution with the same copper concentration only exceeds the solubility limit of $\mathrm{Cu}(\mathrm{OH})_{2}$ at a $\mathrm{pH}$ greater than 
$6.3 .^{80}$

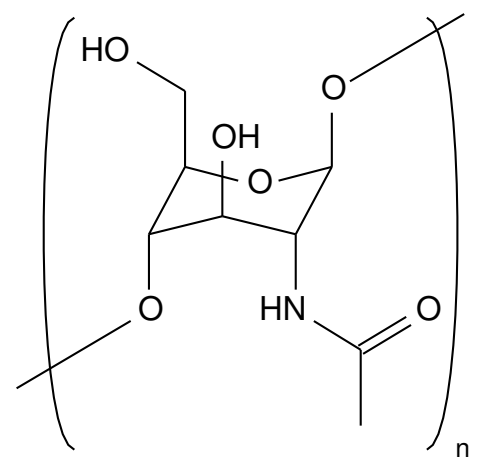

(a)

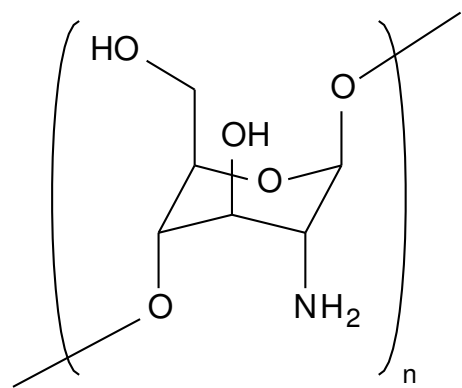

(b)

Figure 3.1 Chitin (a) and its deacetylated analogue, chitosan (b).

Given that the mechanism of bio-sorption is a combination of ion-exchange and coordination between the metal ion and the sorbent, it becomes susceptible to interference due to competition from other species. Scheinder et al., ${ }^{81}$ have observed that the sorption of copper by biomass derived from Potamogeton lucens (an aquatic plant) from a synthetic mining leachate was affected by the presence of the complexing agents $\mathrm{CN}^{-}$and $\mathrm{EDTA}^{4-}$. These species form the anionic copper complexes $\left[\mathrm{Cu}(\mathrm{CN})_{4}\right]^{3-}$ and $[\mathrm{CuEDTA}]^{2-}$, which has two effects on the sorption process:

- The target sorbate becomes anionic (as opposed to the cationic $\left.\left[\mathrm{Cu}\left(\mathrm{H}_{2} \mathrm{O}\right)_{5}\right]^{2+}\right),{ }^{82 \dagger}$ negating any cation ion-exchange mechanism.

- The $\mathrm{CN}^{-}$and $\mathrm{EDTA}^{4-}$ ligands compete with potential ligands in the sorbent, such as carboxylate groups. This retards the potential complexation sorption mechanism (the formation of $\left[\mathrm{Cu}(\mathrm{OOC}-\mathrm{R})_{\mathrm{x}}\right]^{(2-\mathrm{x})+}$ complexes with the sorbent).

It was noted by the authors that copper sorption was reduced by $28 \%$ in the presence of equimolar amounts of $\mathrm{CN}^{-}$, and $97 \%$ in the presence of equimolar amounts of $\mathrm{EDTA}^{4-}$. The presence of equimolar amounts of either $\mathrm{Na}^{+}, \mathrm{Ca}^{2+}, \mathrm{Fe}^{3+}$ or pine oil (a non-ionic surfactant) did not significantly reduce the observed copper sorption of the material.

\footnotetext{
${ }^{\dagger} \mathrm{X}$-ray absorption spectroscopic studies suggest that aqueous $\mathrm{Cu}^{2+}$ exists in a five-coordinate elongated square pyramidal geometry rather than a six-coordinate Jahn-Teller distorted octahedral geometry. ${ }^{82}$
} 


\subsubsection{Sorption by Synthetic Materials}

Even though copper primarily coordinates to oxygen based ligands in biomass, synthetic sorbent materials have been reported that make use of the strong affinity of $\mathrm{Cu}^{2+}$ for nitrogen or sulfur groups. Šljukić et al. ${ }^{83}$ have reported copper uptake by glassy carbon modified by a surface coating of the sulfur-containing amino acid cysteine. Their material exhibited a copper sorption capacity of $0.49 \mathrm{mmol} \mathrm{Cu} \mathrm{g}^{-1}$ and displayed behaviour consistent with the Freundlich isotherm.

Wang and Wang ${ }^{84}$ have developed an amine based co-polymer resin with a chelating core similar to EDTA, and additional side chains containing ethylamine oligomers (Figure 3.2). They reported the copper uptake of their resin as $1.08 \mathrm{mmol} \mathrm{g}^{-1}$, with a high stability constant for the $\mathrm{Cu}$-resin complex $\left(\log K_{S}=18.72\right)$. It was demonstrated that $\mathrm{Cu}^{2+}$ would complex to the resin in preference to EDTA. The authors provided a value of $\log K_{S}=18.30$ for the Cu-EDTA complex, slightly lower than that for their resin.

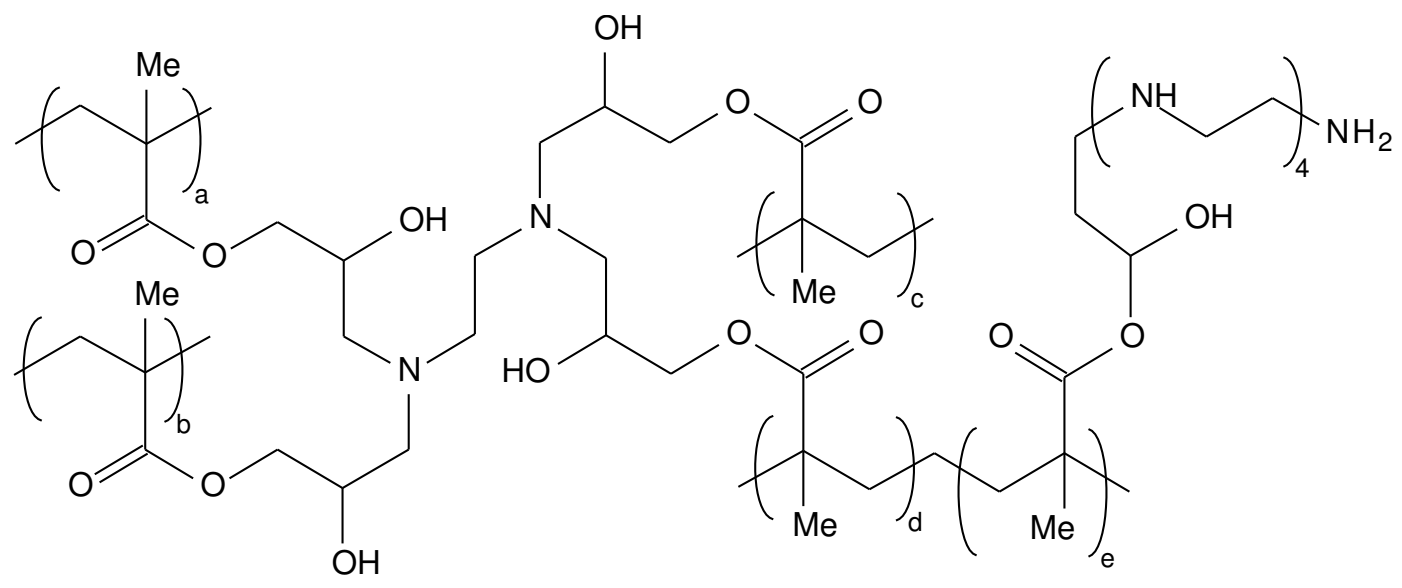

Figure 3.2 Co-polymer resin as used by Wang and Wang for copper sorption. ${ }^{84}$

\subsubsection{Ion Exchange Resins}

Ion exchange resins facilitate the sorption of undesirable species via the exchange of the species of interest for a sacrificial ion within the resin. The ion exchange resins are typically organic resins containing either acidic functional groups (such as $\mathrm{R}-\mathrm{COOH}$ or $\mathrm{R}-\mathrm{SO}_{3} \mathrm{H}$ ) or basic functional groups ( exchange of the sacrificial ion $\left(\mathrm{H}^{+}\right)$for the ion(s) of interest in the influent stream $\left(\mathrm{Cu}^{2+}\right.$ or 
similar). Ion exchange resins containing the acidic functional groups are known as cation exchange resins (exchange of $\mathrm{H}^{+}$), whereas those containing basic functional groups are known as anion exchange resins (exchange of $\mathrm{OH}^{-}$). Formally, ion exchange is a purely electrostatic process; complexation of the influent ion is not necessary. As an electrostatic process, the exchange favours ions of higher charge. Thus, $\mathrm{Fe}^{3+}$ would be favoured over $\mathrm{Cu}^{2+}$ which in turn would be favoured over $\mathrm{Na}^{+}$. The formation of complexes between the resin and exchanged ion may cause ions of lower charge to become favoured.

Many zeolites can act as cation exchangers. Due to the substitution of tetrahedral $\mathrm{AlO}_{4}^{5-}$ for $\mathrm{SiO}_{4}^{4-}$ within the zeolite structure, charge-balancing cations are required. These cations (often alkali) are potentially exchangeable. For example, sodium mordenite, a zeolite with a Si:Al ratio of 10:1 may exchange the sodium within its structure for copper, as reported by Kuroda et al. ${ }^{85,86}$ The authors observed that copper (but not calcium or nickel) will ion-exchange in sodium mordenite to a greater extent than that predicted by charge balance (one $\mathrm{Cu}^{2+}$ ion exchanging for two $\mathrm{Na}^{+}$ions). ${ }^{\dagger}$ Ion exchange with $\mathrm{Ca}^{2+}$ or $\mathrm{Ni}^{2+}$ still occurred, just not to the same extent. An exchange capacity for copper of $215 \%$ was recorded after 15 exchange cycles. Although not reported on a per gram basis, the $215 \%$ exchange capacity equates to approximately $2.4 \mathrm{mmol} \mathrm{Cu}^{2+} \mathrm{g}^{-1}$ (based on an empirical formula for sodium mordenite of $\mathrm{NaAlSi}_{10} \mathrm{O}_{22} \ddagger$ ). It was demonstrated by the authors that copper may form hydroxyl-bridged dimers (Figure 3.3), something not observed with the $\mathrm{Ca}^{2+}$ or $\mathrm{Ni}^{2+}$ metal ions. They attributed this to the greater degree of<smiles>OC(O)C(O)OC1(O)CCCC1</smiles>

Figure 3.3 Hydroxide bridged copper dimer, as observed by Kurdoa et al. ${ }^{85}$

\footnotetext{
$\dagger$ A $100 \%$ exchange capacity would be reported if one $\mathrm{Cu}^{2+}$ ion was sorbed for every two $\mathrm{Na}^{+}$ions initially present in the zeolite. If the sorbed species was instead $\left[\mathrm{Cu}_{2}(\mathrm{OH})_{2}\left(\mathrm{H}_{2} \mathrm{O}\right)_{4}\right]^{2+}$ (Figure 3.3), the effective charge per copper ion would decrease. As such, an exchange capacity in excess of $100 \%$ becomes possible. ${ }^{*}$ Although Kuroda et al. ${ }^{85}$ report using a mordenite with a Si:Al ratio of 10:1, sodium mordenite XRD patterns registered in the ICDD Powder Diffraction File ${ }^{41}$ list chemical formula of $\mathrm{Na}_{2} \mathrm{Al}_{2} \mathrm{Si}_{13.3} \mathrm{O}_{26.6+\mathrm{x}}$ (PDF 00-049-0924) or $\mathrm{Na}_{0.014} \mathrm{Al}_{0.1646} \mathrm{Si}_{0.8354} \mathrm{O}_{2} \mathrm{H}_{0.16}$ (PDF 01-070-1833). These minerals have Si:Al ratios of $6.65: 1$ and 5.08:1 respectively.
} 
hydrolysis for copper:

$$
\left[\mathrm{M}\left(\mathrm{H}_{2} \mathrm{O}\right)_{\mathrm{n}}\right]^{2+} \stackrel{K}{\rightleftharpoons}\left[\mathrm{M}\left(\mathrm{H}_{2} \mathrm{O}\right)_{\mathrm{n}-1}(\mathrm{OH})\right]^{+}+\mathrm{H}^{+}
$$

Kuroda et al. ${ }^{85}$ provided $\mathrm{p} K$ values for $\mathrm{Cu}^{2+}, \mathrm{Ni}^{2+}$ and $\mathrm{Ca}^{2+}$ of $8,10.5$ and 12.85 respectively. Thus, the equilibrium in (3.3) lies further to the right for $\mathrm{Cu}^{2+}$ than it does for $\mathrm{Ni}^{2+}$ or $\mathrm{Ca}^{2+}$.

Copper sorption by zeolites is often studied both as a means of wastewater purification $^{62,87-90}$ and as a means of creating potential catalytic materials. ${ }^{91-93}$

\subsubsection{Silicate based Sorbents}

Cement (consisting of mainly calcium silicate hydrate phases) ${ }^{23}$ has a long history of being used for the encapsulation of waste species (typically radionuclides, but also other toxic metal species) in the form of concretes. ${ }^{94,95}$ During the ageing of the concrete, the waste species are incorporated within the material. The stability of such composite materials towards leaching has been attributed to the basic nature of the calcium silicate hydrate phases such as jennite or tobermorite or the calcium aluminate sulfate phase ettringite present in the concrete. The low solubility of many metal hydroxides means that they are not leached from the concrete. In a review of waste immobilisation by cements, Gougar et al. ${ }^{94}$ noted that many waste species would substitute within the crystal lattice of ettringite $\left(3 \mathrm{CaO} \cdot \mathrm{Al}_{2} \mathrm{O}_{3} \cdot 3 \mathrm{CaSO}_{4} \cdot 32 \mathrm{H}_{2} \mathrm{O}\right)$. For example: $\mathrm{Sr}^{2+}, \mathrm{Pb}^{2+}, \mathrm{Cd}^{2+}$ etc. may occupy octahedral $\mathrm{Ca}^{2+}$ sites; $\mathrm{Cr}^{3+}, \mathrm{Fe}^{3+}, \mathrm{Ni}^{3+},{ }^{\dagger}$ etc. may occupy tetrahedral $\mathrm{Al}^{3+}$ sites; $\mathrm{AsO}_{4}^{-} \mathrm{CrO}_{4}^{-}, \mathrm{B}(\mathrm{OH})_{4}^{-}$, etc. may occupy $\mathrm{SO}_{4}^{2-}$ sites.

While the use of encapsulation techniques was developed concurrently with the nuclear industry in the 1940s and 1950s, the technique was usually applied as a method to dispose of small volumes of concentrated waste. More recently, studies have been reported on the use of concrete (or concrete derived products) as sorbent materials for treating more dilute waste streams. ${ }^{96-99}$ Coleman et al., for example, reported the use of granulated concrete

\footnotetext{
${ }^{\dagger}$ Although nickel is not often found in the +3 oxidation state, it may occur in nuclear waste streams. ${ }^{94}$
} 
as a sorbent material for copper, lead and zinc. ${ }^{96}$ The sorption capacity of their material towards $\mathrm{Cu}^{2+}$ was reported as $0.55 \mathrm{mmol} \mathrm{g}^{-1}$. Although the study involved a laboratoryprepared sorbent, it was indicated that the principles could easily be applied to material derived from construction industry waste. 


\subsection{Results}

The use of NCS as a sorbent material for copper, as either the chloride or nitrate salts, was investigated. The kinetics of the sorption reaction were determined, and the nature of the sorbed products were characterised. Based on these findings, possible mechanisms for the sorption process were proposed.

\subsubsection{Sorption Studies}

For comparison purposes, Figure 3.4 shows the behaviour of NCS when permitted to equilibrate with water. It can be seen from the figure that the $\mathrm{pH}$ reaches an equilibrium value of approximately 10 within 30 minutes, however calcium continues to be leached into the solution. The calcium concentration, after four hours, was determined as $1.33 \pm 0.03 \mathrm{mmol} \mathrm{dm}^{-3}$. At this point, the $\mathrm{pH}$ of the solution had increased from 7.2 to 10.0 , equivalent to a final hydroxide ion concentration of $0.1 \mathrm{mmol} \mathrm{dm}^{-3}$.
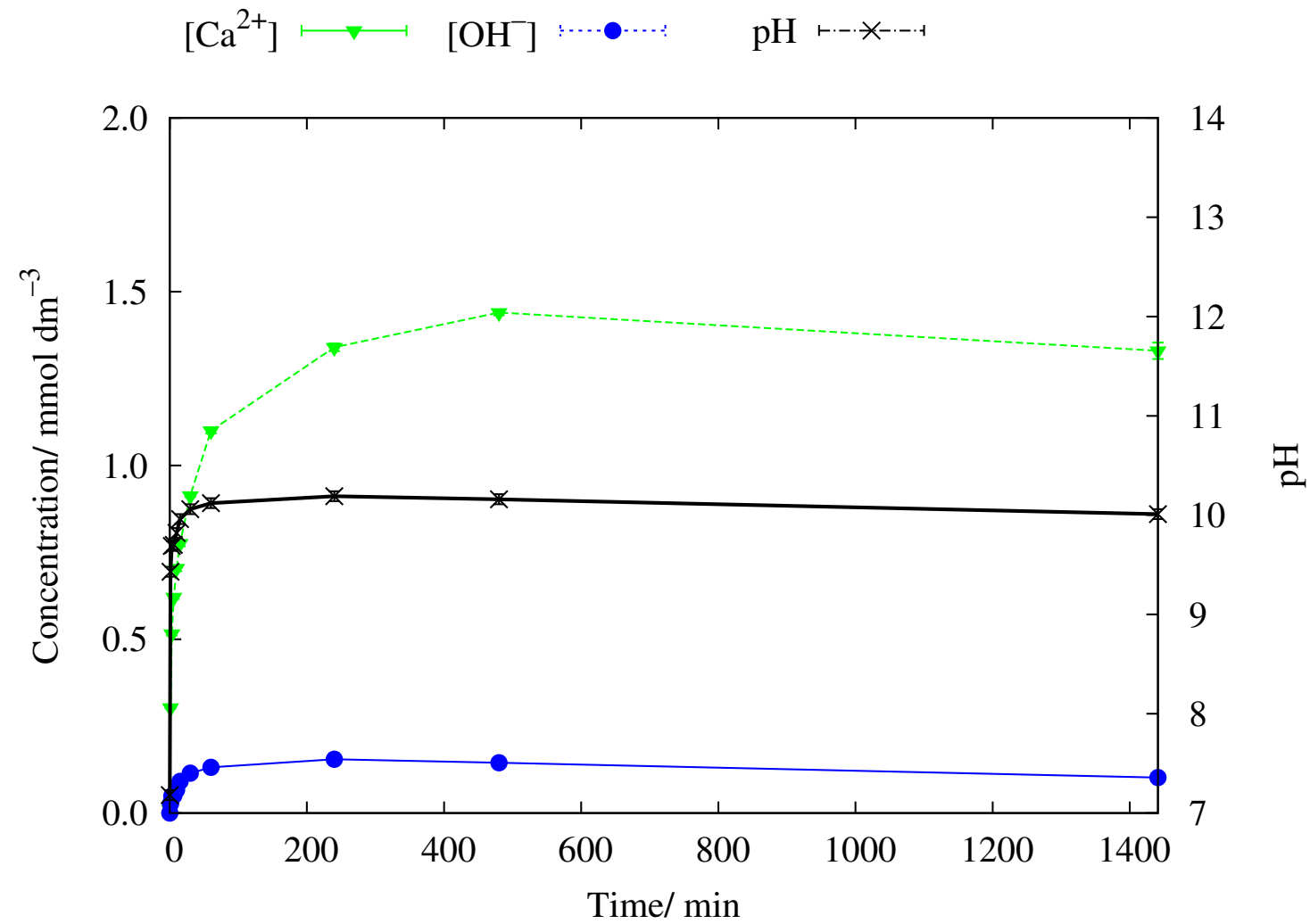

Figure 3.4 Leaching of $\mathrm{Ca}^{2+}$ from NCS in contact with water. 2-Ethoxyethanol washed NCS applied at a loading of $1 \mathrm{~g} \mathrm{dm}^{-3}$.

Figure 3.5 shows the sorption characteristics of 2-ethoxyethanol washed NCS towards 
copper as either the chloride or nitrate salts at a high initial copper concentration of approximately $80 \mathrm{mmol} \mathrm{dm}^{-3}$, with NCS applied at a loading of $1 \mathrm{~g} \mathrm{dm}^{-3}$. It can be seen from the figure that there is no substantive difference between the copper salts. The sorption capacity of NCS, as determined from these results is $9 \pm 1 \mathrm{mmol} \mathrm{Cu}^{2+} \mathrm{g}^{-1}$ for $\mathrm{CuCl}_{2}$ and $10 \pm 1 \mathrm{mmol} \mathrm{Cu}{ }^{2+} \mathrm{g}^{-1}$ for $\mathrm{Cu}\left(\mathrm{NO}_{3}\right)_{2}$. The sorption of copper is associated with a release of calcium from the NCS into solution, although at the high copper concentrations employed this does not significantly alter the $\mathrm{pH}$ of the solution. This is expected should the $\mathrm{pH}$ value of the solution be determined by the solubility of $\mathrm{Cu}(\mathrm{OH})_{2}$. If the solution hydroxide concentration is determined by the $\mathrm{Cu}(\mathrm{OH})_{2} \rightleftharpoons \mathrm{Cu}^{2+}+2 \mathrm{OH}^{-}$

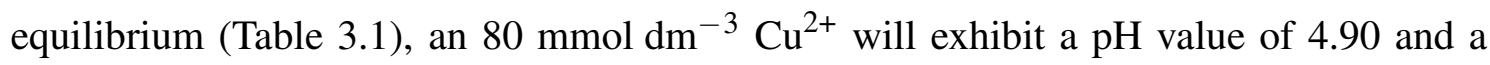

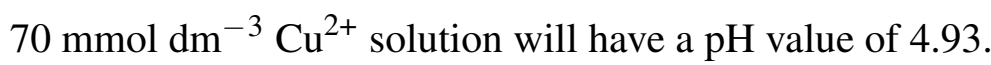

Equilibrium in Figure 3.5 is attained after approximately one hour. At this stage most of the calcium has been leached from the NCS: the $\mathrm{Ca}^{2+}$ concentration at equilibrium is $6.5 \pm 0.1 \mathrm{mmol} \mathrm{dm}^{-3}$ in the case of Figure $3.5(\mathrm{a})\left(\mathrm{CuCl}_{2}\right)$ and $5.6 \pm 0.3 \mathrm{mmol} \mathrm{dm}^{-3}$ in the case of Figure 3.5(b) $\left(\mathrm{Cu}\left(\mathrm{NO}_{3}\right)_{2}\right)$. This amount leached is equivalent to between 31-38 wt \% $\mathrm{CaO}$ in the initial NCS. This corresponds well to the 38 wt $\% \mathrm{CaO}$ given for a typical elemental analysis in Table $1.4 .^{\dagger}$

Additional sorption data are presented in Figure 3.6. An experiment in which the initial $\mathrm{Cu}\left(\mathrm{NO}_{3}\right)_{2}$ concentration was approximately $8 \mathrm{mmol} \mathrm{dm}^{-3}$ (Figure 3.6(a)), shows that the copper sorption is incomplete even after 1 day (1440 minutes). The copper concentration in solution at this time is $2.04 \pm 0.03 \mathrm{mmol} \mathrm{dm}^{-3}$, equivalent to a $74 \%$ copper removal. By this point, however, the calcium concentration of the solution has increased to $6.2 \pm 0.1 \mathrm{mmol} \mathrm{dm}^{-3}$, indicating that most of the calcium initially present in the NCS has been leached out.

In Figures 3.6(b)-(c), plots for experiments are presented where the initial copper concentration was approximately $1.6 \mathrm{mmol} \mathrm{dm}^{-3}$. No copper can be detected in solution within 60 minutes (the recorded copper concentration is of a lesser magnitude than the

\footnotetext{
${ }^{\dagger}$ As all the calcium present in NCS can be leached into solution during copper uptake, it is likely that the process investigated here is not a true sorption mechanism. For a sorption process, it would be expected that only the surface-available would react. Although this may be the case at low copper concentrations, it is possible to leach all calcium from the NCS material, when an excess of copper is present. In effect, this degrades the NCS. For simplicity in this work the term sorption will be used to refer to both mechanisms.
} 


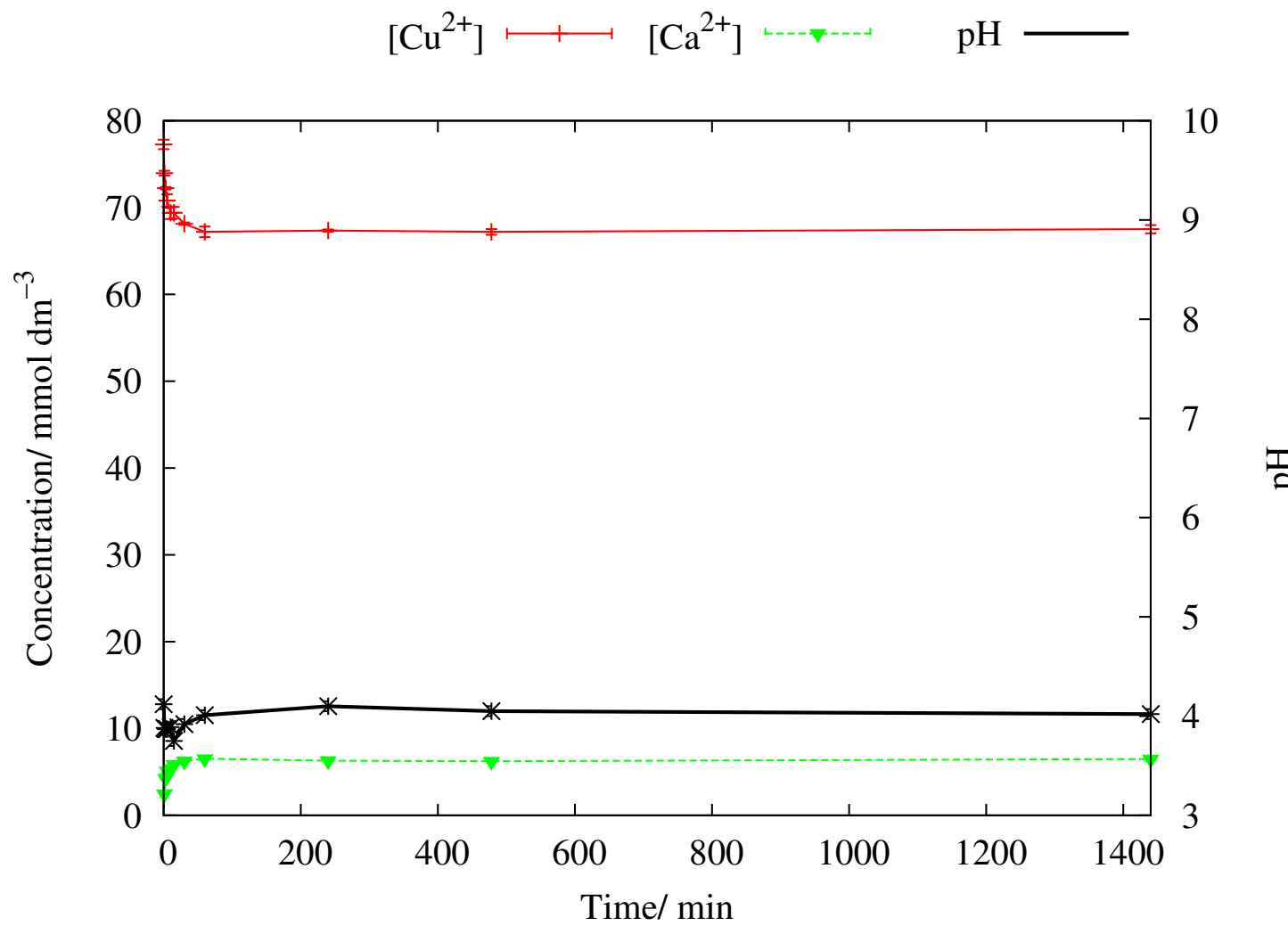

(a)

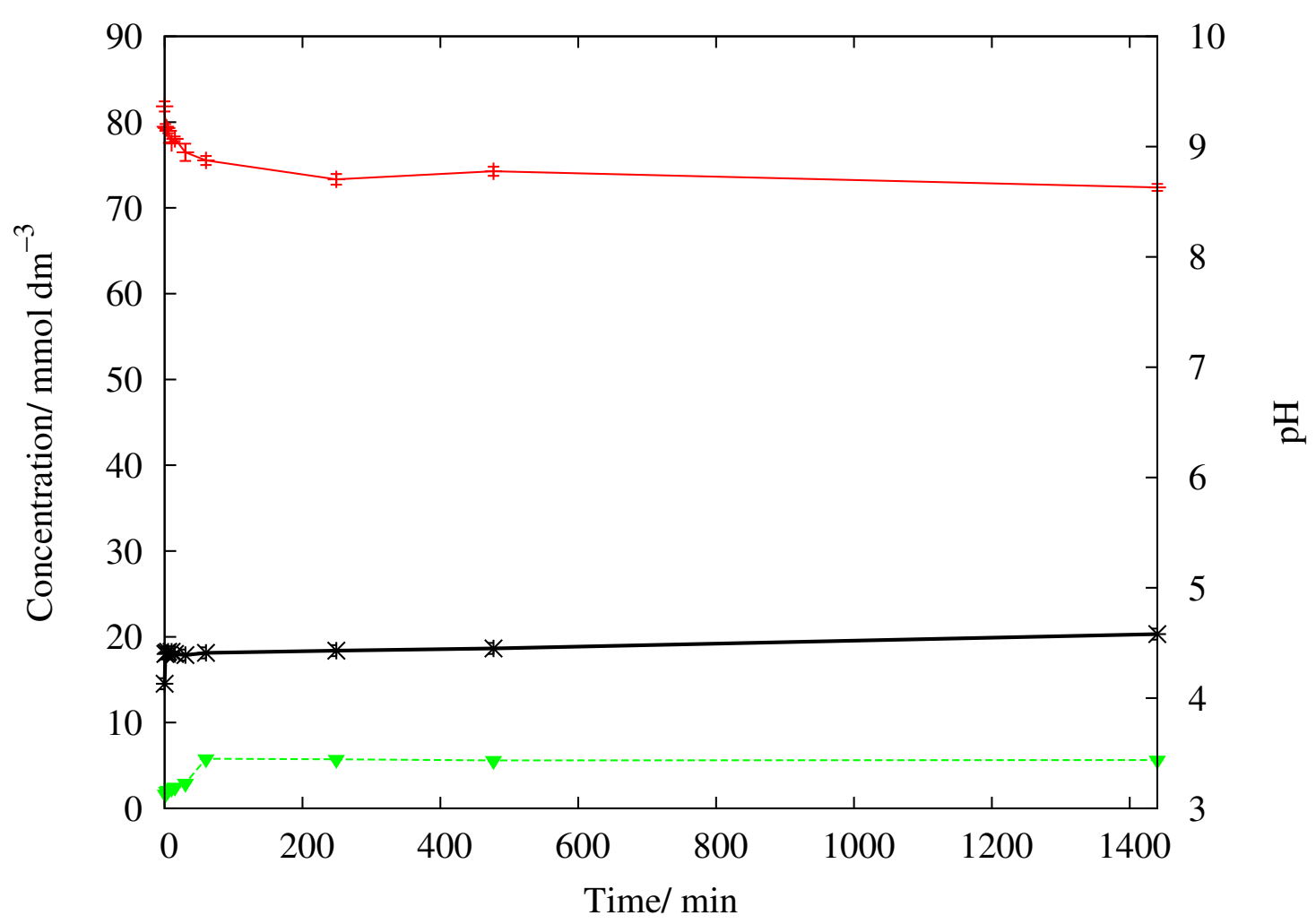

(b)

Figure 3.5 Sorption characteristics of NCS towards $\mathrm{Cu}^{2+}$ as either $\mathrm{CuCl}_{2}$ or $\mathrm{Cu}\left(\mathrm{NO}_{3}\right)_{2}$ an an initial concentration of approximately $80 \mathrm{mmol} \mathrm{dm}^{-3}$ : (a) $\mathrm{CuCl}_{2}$; (b) $\mathrm{Cu}\left(\mathrm{NO}_{3}\right)_{2}$. 2-Ethoxyethanol washed NCS applied at a loading of $1 \mathrm{~g} \mathrm{dm}^{-3}$. 


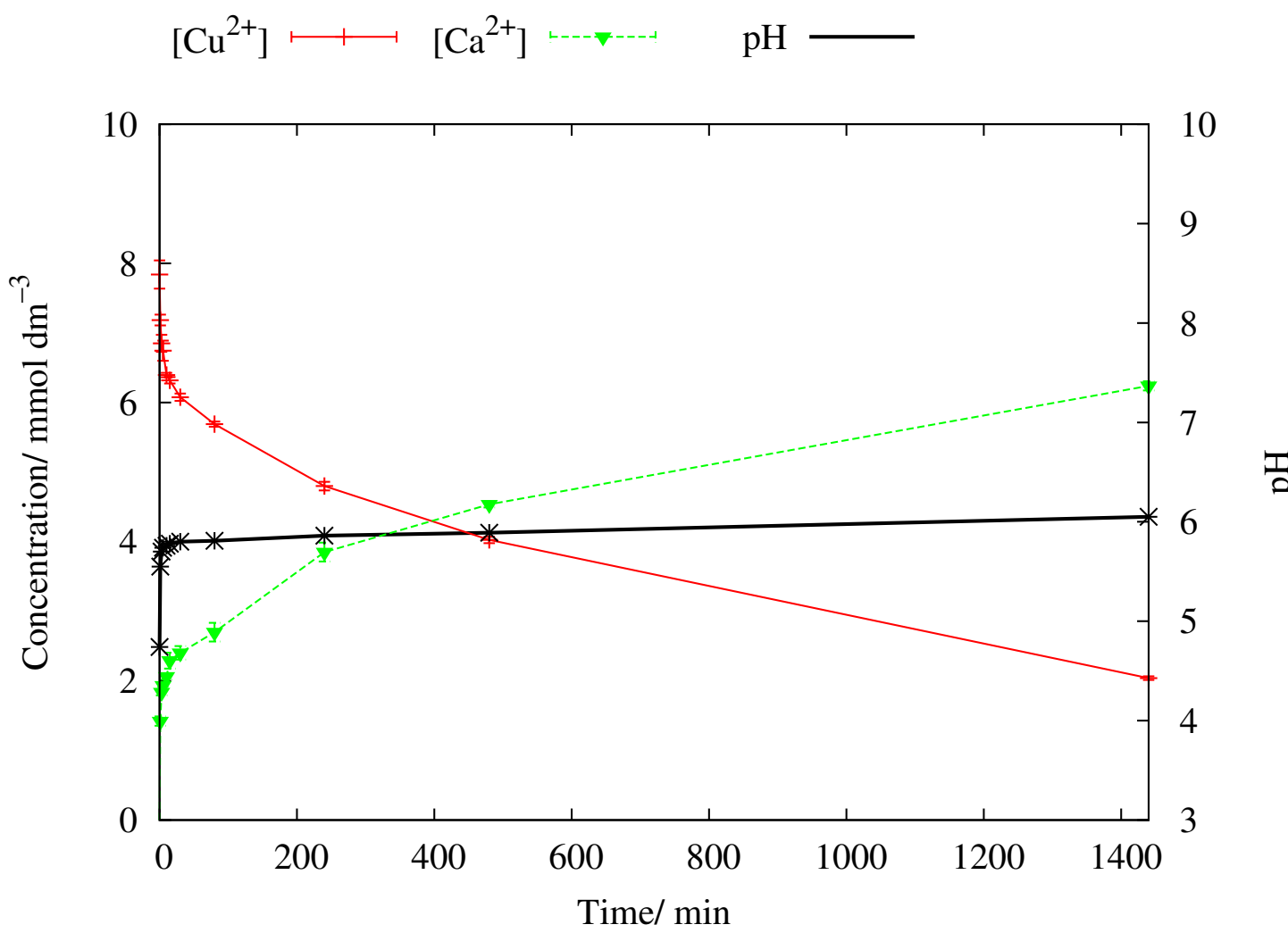

(a)

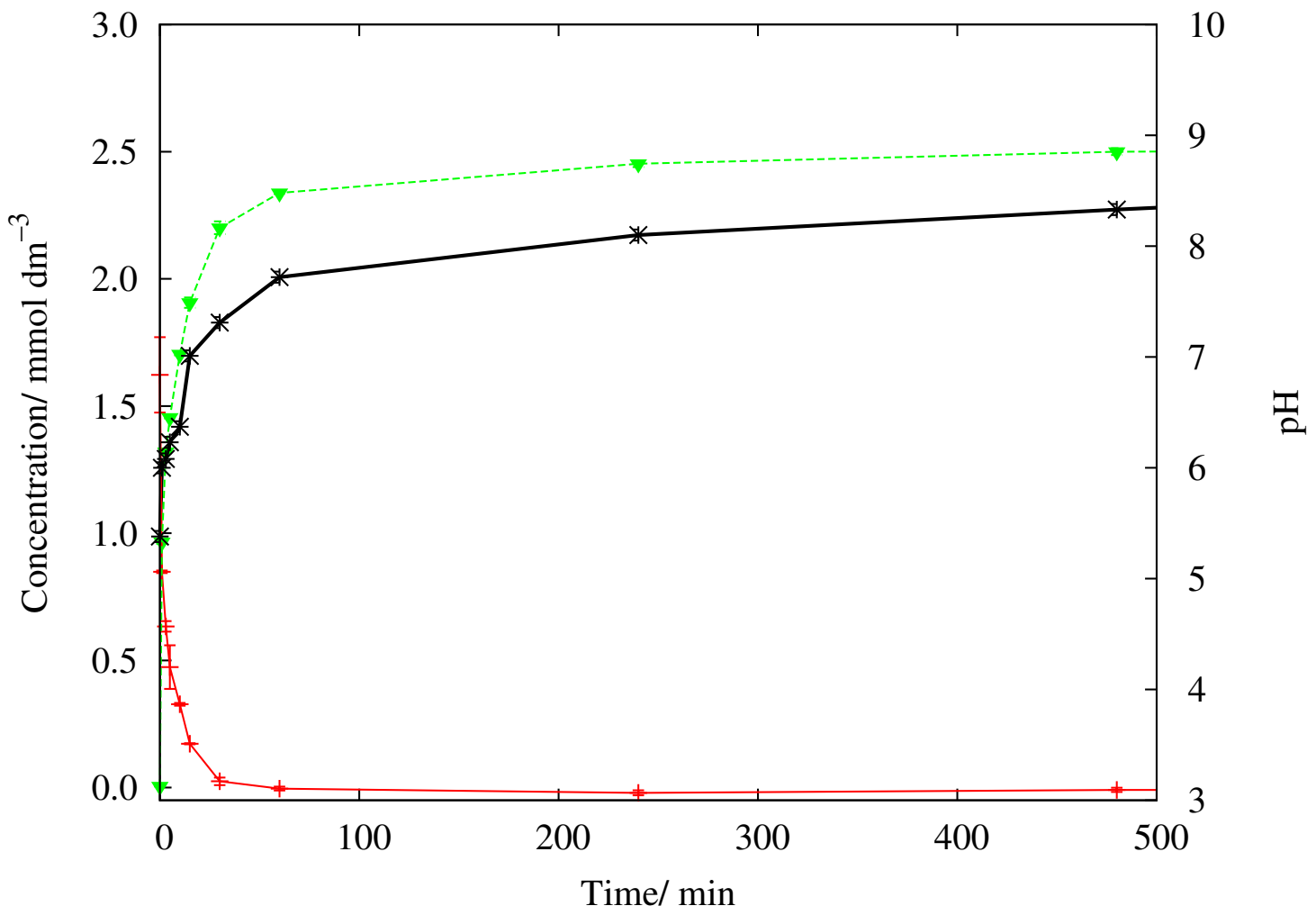

(b)

Figure 3.6 First part of Figure 3.6. (Figure continued over page.) 


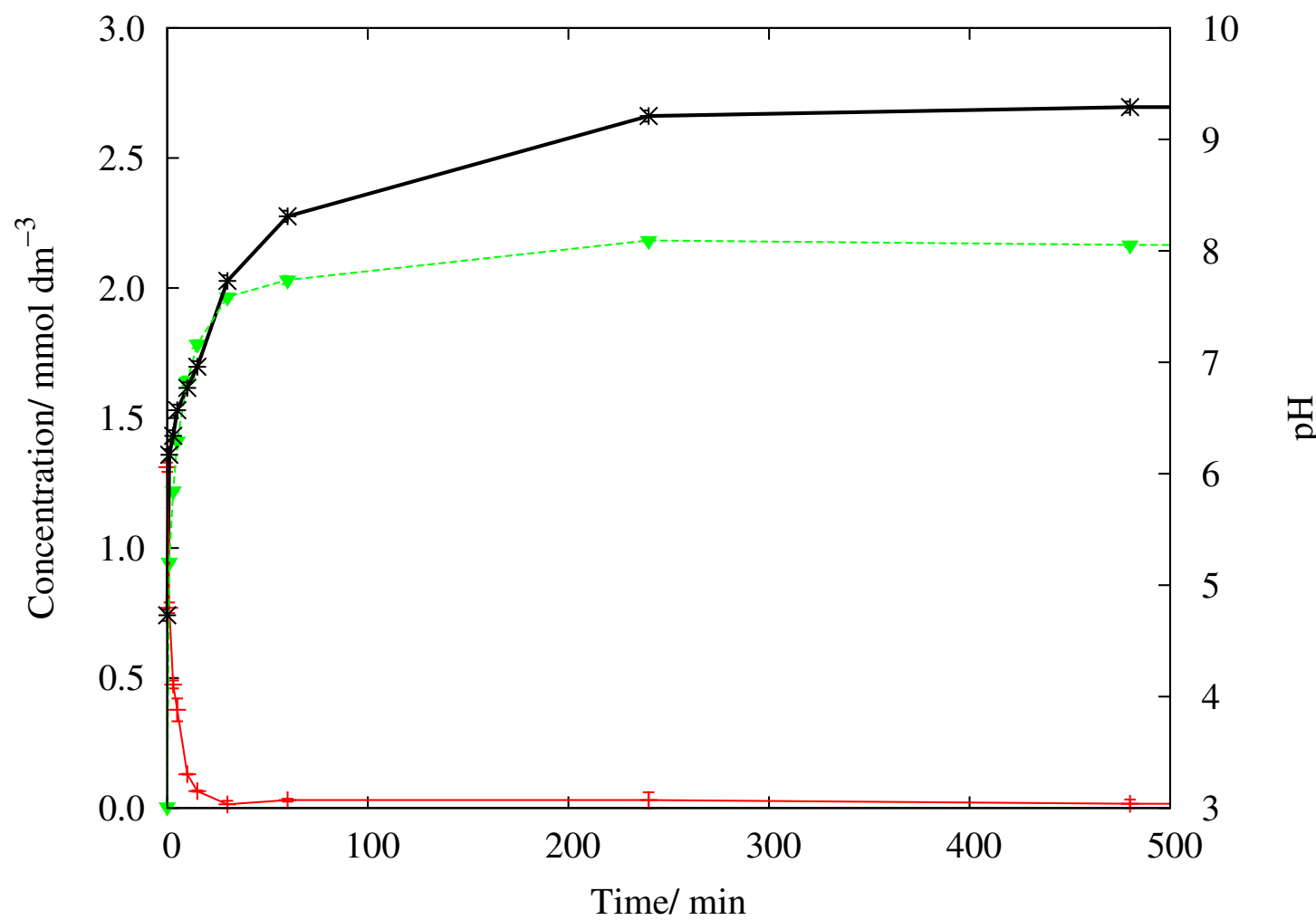

(c)

Figure 3.6 Sorption characteristics of NCS towards copper salts at lower concentrations: (a)-(b) $\mathrm{Cu}\left(\mathrm{NO}_{3}\right)_{2}$; (c) $\mathrm{CuCl}_{2}$. 2-Ethoxyethanol washed NCS applied at a loading of $1 \mathrm{~g} \mathrm{dm}^{-3}$.

$8 \mu \mathrm{mol} \mathrm{dm}{ }^{-3}$ uncertainty). This equates to a copper removal greater than $99.5 \%$. The slightly lower $\mathrm{pH}$ value observed in Figure 3.6(b) (when compared with (c)) may be attributed to the higher initial copper concentration. Although it is not shown in the figure, after one day (1440 minutes) had elapsed, both solutions exhibited a similar $\mathrm{pH}$ value, in the range of 9.1-9.2.

\subsubsection{Sorption by Derivatives of NCS}

To test the necessity for calcium within the NCS structure to achieve copper sorption, tests were conducted with pH-adjusted NCS. From Figure 3.7, it can be seen that as the calcium content of the NCS is reduced, so is the copper uptake capacity. The maximum copper uptake capacity, calculated from the data presented in Figure 3.7(a), is $2 \pm 1 \mathrm{mmol} \mathrm{g}^{-1}$. Calcium released from the material provided a final $\mathrm{Ca}^{2+}$ concentration of $1.37 \pm 0.04 \mathrm{mmol} \mathrm{dm}^{-3}$. This is equivalent to $7.44 \mathrm{wt} \% \mathrm{CaO}$ in the initial sorbent. 


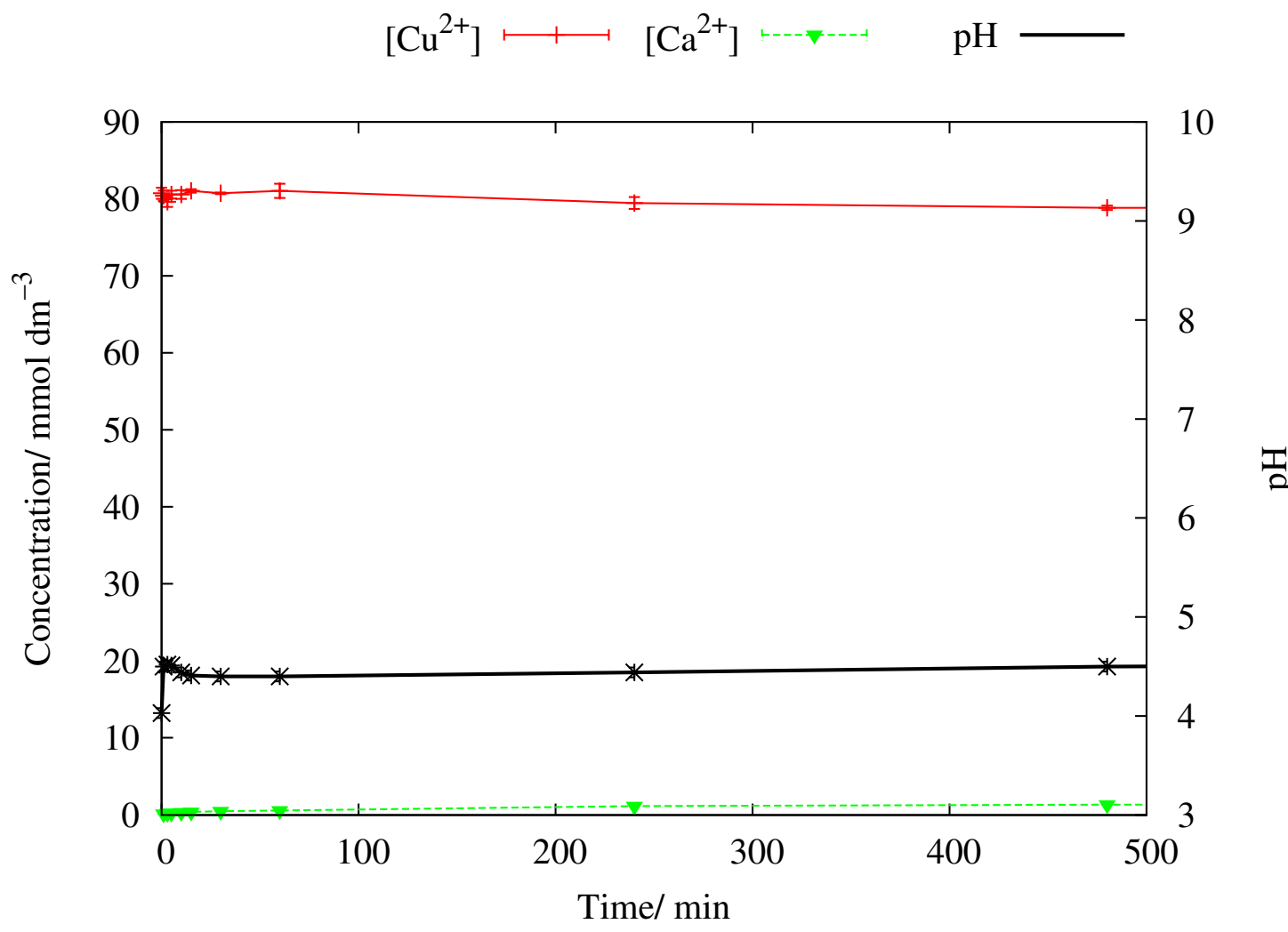

(a)

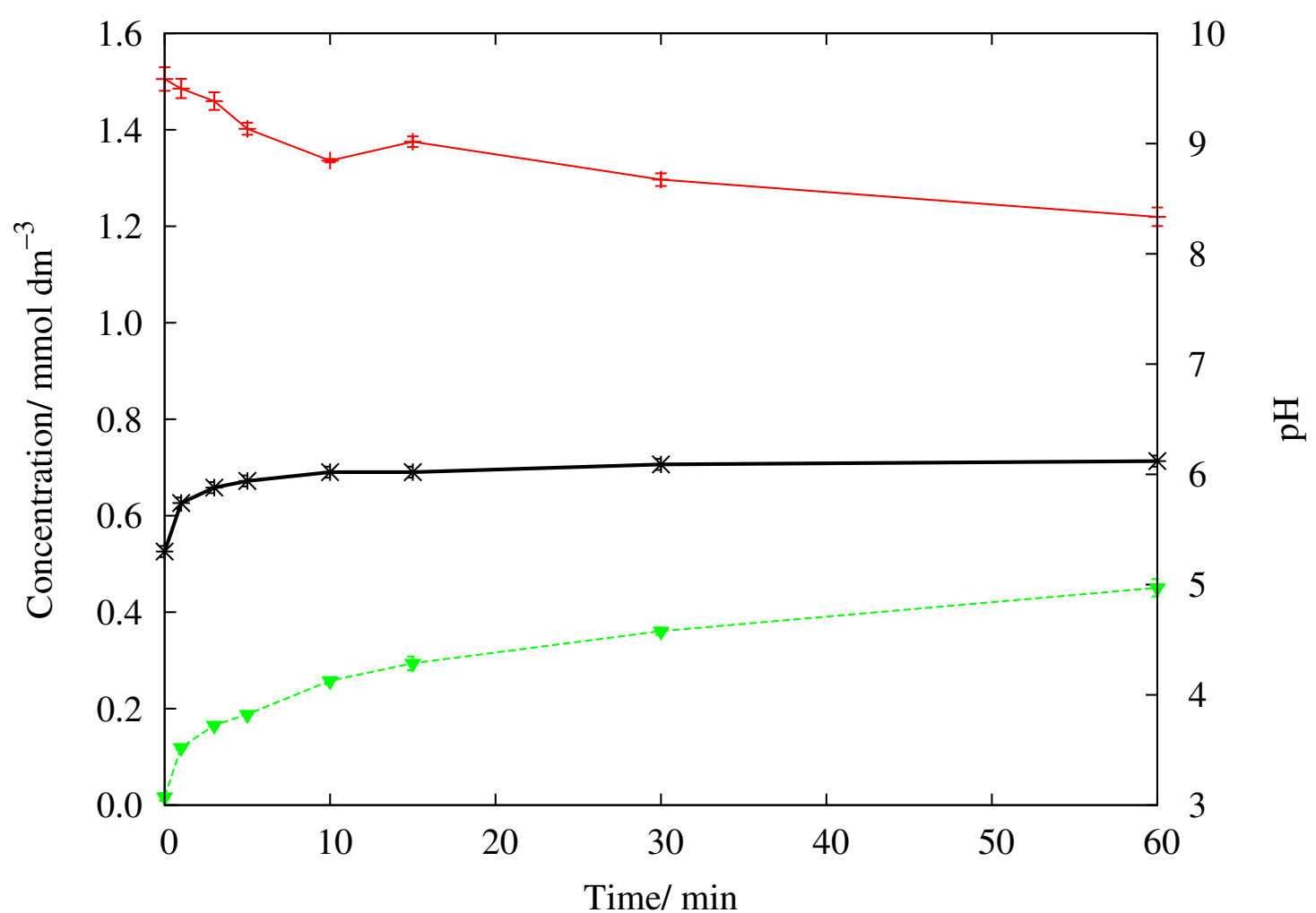

(b)

Figure 3.7 First part of Figure 3.7. (Figure continued over page.) 


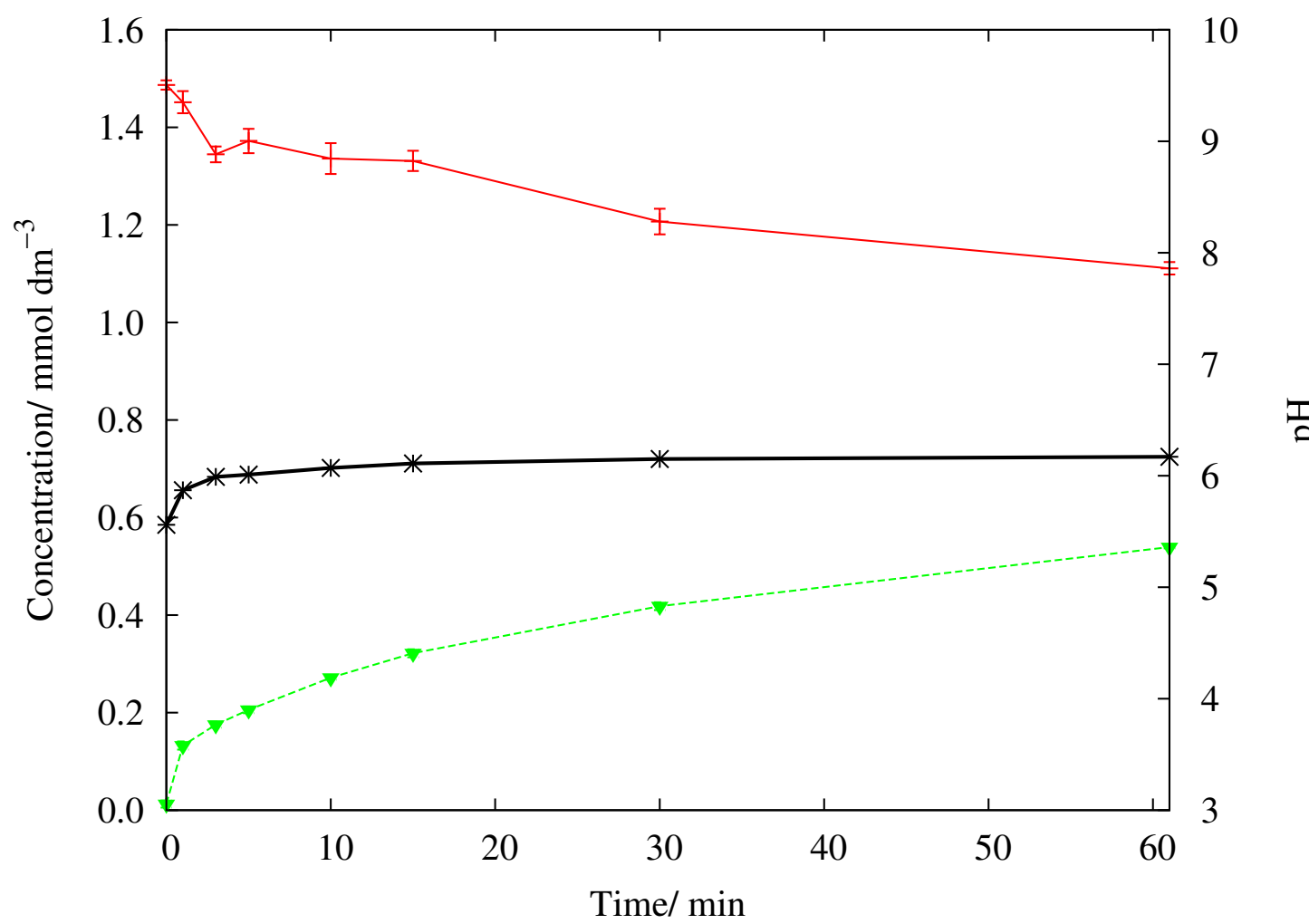

(c)

Figure 3.7 Sorption characteristics of $\mathrm{pH}$ adjusted NCS towards copper salts: (a)-(b) $\mathrm{Cu}\left(\mathrm{NO}_{3}\right)_{2} ;$; (c) $\mathrm{CuCl}_{2} \cdot \mathrm{pH}$ adjusted NCS (acid leached) applied at a loading of $1 \mathrm{~g} \mathrm{dm}^{-3}$.

\subsubsection{Competition of Iodine}

As has previously been demonstrated, NCS has a high affinity towards $\mathrm{I}_{2} \cdot{ }^{100}$ Thus, it was thought that the presence of iodine in solution could retard the release of calcium, and hence the copper uptake. To this end, the sorption characteristics of NCS were determined from a solution containing $1.57 \mathrm{mmol} \mathrm{dm}{ }^{-3} \mathrm{CuCl}_{2}, 0.4 \mathrm{mmol} \mathrm{dm}^{-3} \mathrm{I}_{2}$ and $0.7 \mathrm{mmol} \mathrm{dm}^{-3} \mathrm{NaI}$. As seen in Figure 3.8, the concentration of $\mathrm{I}_{2}$ in solution quickly reduces to approximately $0.05 \mathrm{mmol} \mathrm{dm}^{-3}$.

It can also be seen in the figure that calcium is released at a faster rate from the NCS when iodine is present in the system. Copper, when in the +2 oxidation state, is known to react with iodide according to the equation:

$$
2 \mathrm{Cu}^{2+}+5 \mathrm{I}^{-} \rightleftharpoons 2 \mathrm{CuI}(\mathrm{s})+\mathrm{I}_{3}^{-}
$$

The reaction has an electrochemical potential of $\mathrm{E}^{\ominus}=+0.33 \mathrm{~V},{ }^{50}$ and, although no $\mathrm{CuI}$ 


$$
\begin{array}{ll}
{\left[\mathrm{Cu}^{2+}\right], \text { iodine }} & {\left[\mathrm{Cu}^{2+}\right], \text { no iodine }} \\
{\left[\mathrm{Ca}^{2+}\right] \text {, iodine }} & {\left[\mathrm{Ca}^{2+}\right] \text {, no iodine }}
\end{array}
$$

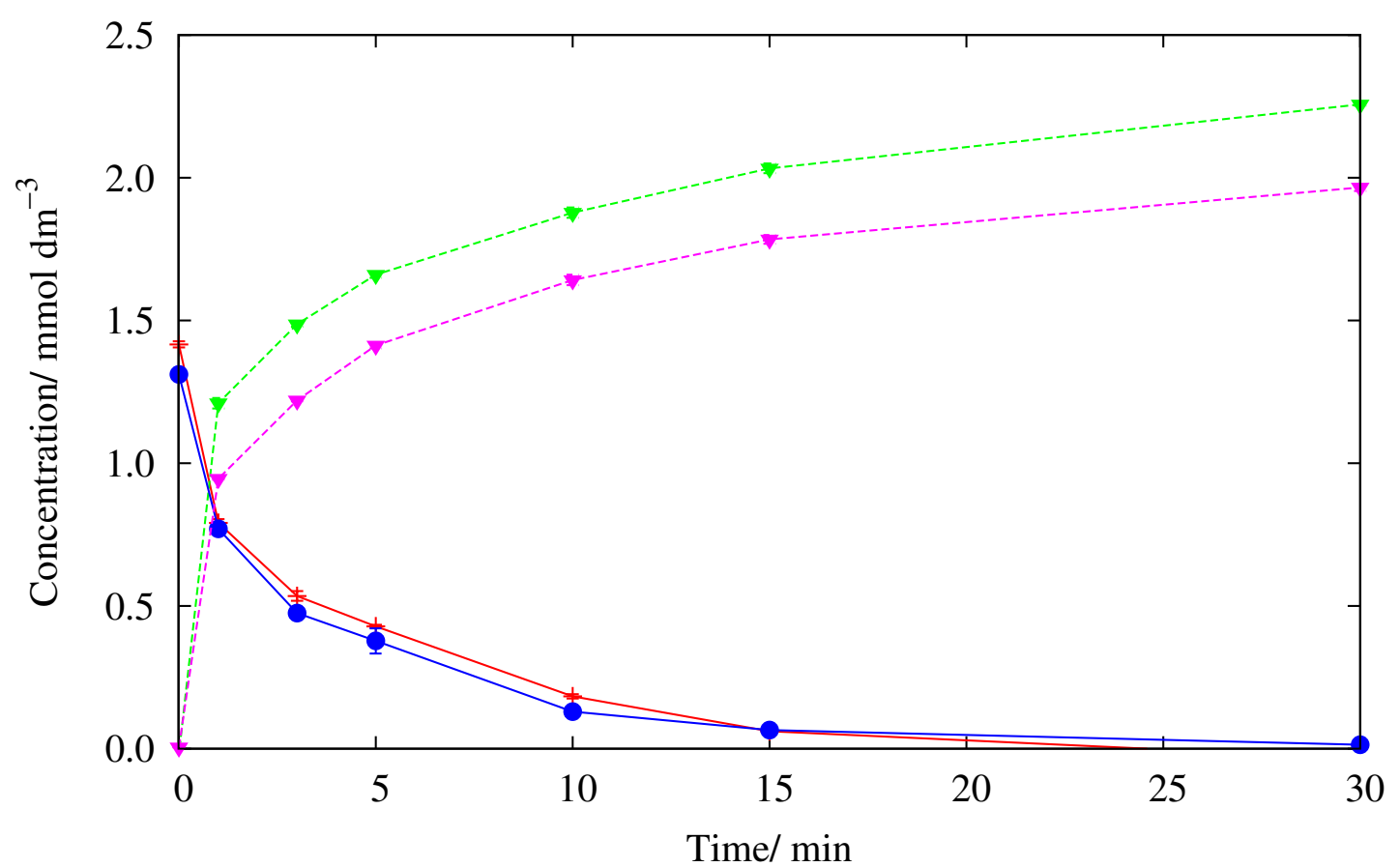

(a)

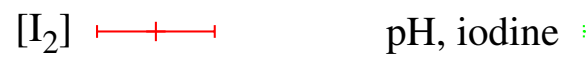

$\mathrm{pH}$, no iodine

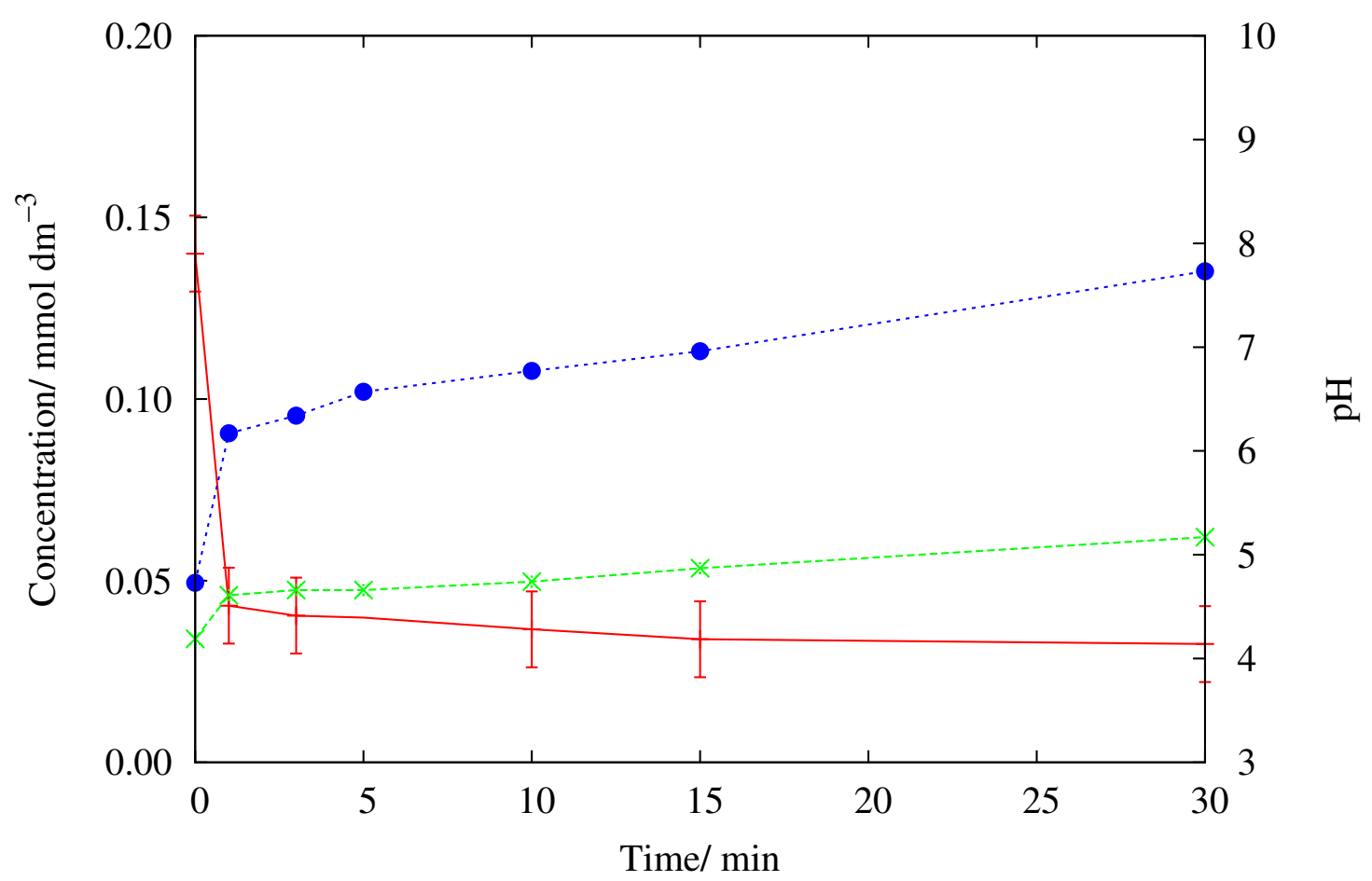

(b)

Figure 3.8 Sorption characteristics of NCS towards $\mathrm{Cu}^{2+}$ in the presence of $\mathrm{I}_{3}^{-}$ $\left(1.57 \mathrm{mmol} \mathrm{dm}^{-3} \mathrm{CuCl}_{2}\right.$ in a solution containing $0.4 \mathrm{mmol} \mathrm{dm}^{-3} \mathrm{I}_{2}$ and $0.7 \mathrm{mmol} \mathrm{dm}^{-3}$ $\left.\mathrm{I}^{-}\right)$. The data referred to as 'no iodine' by the figure legend are taken from Figure 3.6(c), a sorption experiment conducted in the absence of iodine species: (a) effect of $\mathrm{I}_{3}^{-}$on copper uptake and calcium release; (b) effect of $\mathrm{I}_{3}^{-}$on $\mathrm{pH}$ values. 2-Ethoxyethanol washed NCS applied at a loading of $1 \mathrm{~g} \mathrm{dm}^{-3}$. 
precipitate was observed in the solution prior to addition of NCS, such a precipitate could be formed through the addition of excess $\mathrm{I}^{-}$. With the concentration of $\mathrm{I}^{-}$and $\mathrm{I}_{2}$ used it is likely that there was insufficient free $\mathrm{I}^{-}$present to facilitate the reduction of $\mathrm{Cu}^{2+}$, rather the $\mathrm{I}^{-}$stabilised the $\mathrm{I}_{2}$ :

$$
\mathrm{I}^{-}+\mathrm{I}_{2} \rightleftharpoons \mathrm{I}_{3}^{-}
$$

The presence of $\mathrm{Cu}^{2+}$ in these solutions has also been confirmed by EPR spectroscopy. ${ }^{101}$ This suggests that no substantive reduction of copper from the +2 oxidation state to the +1 oxidation state is occurring.

The discrepancy between copper uptake of NCS in the presence and absence of iodine is most likely caused by the iodine coordinating to the calcium, thereby reducing the strength of the bonding between the calcium and the NCS. Although the presence of iodine promotes calcium release from the NCS, it also decreases the rate at which the solution $\mathrm{pH}$ increases. This indicates that the calcium release does not occur via an ion exchange mechanism with $\mathrm{H}^{+}$, such as: ${ }^{\dagger}$

$$
(\mathrm{CaO})_{0.8} \mathrm{SiO}_{2}+2 x \mathrm{H}^{+} \rightleftharpoons(\mathrm{CaO})_{(0.8-x)}(\mathrm{OH})_{2 x} \mathrm{SiO}_{(2-x)}+x \mathrm{Ca}^{2+}
$$

What is more likely is that the interaction of iodine (or $\mathrm{I}_{3}^{-}$) with $\mathrm{NCS}^{32}$ promotes release of $\mathrm{Ca}^{2+}$ from the NCS, leaving the NCS with a negative surface charge. Reaction of this negatively charged NCS with $\mathrm{H}^{+}$(which leads to an increase in the solution $\mathrm{pH}$ ) is retarded in the presence of iodine.

$$
\begin{array}{r}
(\mathrm{CaO})_{0.8} \mathrm{SiO}_{2}+y\left[\mathrm{I}_{2}, \mathrm{I}_{3}^{-}\right] \rightleftharpoons(\mathrm{CaO})_{(0.8-x)} \mathrm{O}_{x} \mathrm{SiO}_{2}\left(\left[\mathrm{I}_{2}, \mathrm{I}_{3}^{-}\right]\right)_{y}^{x-}+x \mathrm{Ca}^{2+} \\
(\mathrm{CaO})_{(0.8-x)} \mathrm{O}_{x} \mathrm{SiO}_{2}\left(\left[\mathrm{I}_{2}, \mathrm{I}_{3}^{-}\right]\right)_{y}^{x-}+2 x \mathrm{H}^{+} \longrightarrow(\mathrm{CaO})_{(0.8-x)}(\mathrm{OH})_{2 x} \mathrm{SiO}_{(2-x)}\left(\left[\mathrm{I}_{2}, \mathrm{I}_{3}^{-}\right]\right)_{y}
\end{array}
$$

The presence of a negatively charged silicate surface leads to copper sorption. In effect,

${ }^{\dagger}$ For reasons of clarity, the water coordinated to calcium in NCS (see Figure 1.2) is not shown. Thus, NCS is represented here as $(\mathrm{CaO})_{0.8} \mathrm{SiO}_{2}$, rather than $\mathrm{Ca}_{0.8} \mathrm{SiO}_{2.8} \cdot 2 \mathrm{H}_{2} \mathrm{O}$. 
the calcium ions exchange with the copper ions in solution. In Figure 3.8, it can be seen that the presence of iodine does not substantially effect the rate at which copper is removed from solution. Under conditions for which these sorption experiments were conducted, it is likely that the rate of copper sorption was diffusion controlled.

\subsubsection{Kinetics of Sorption}

The data presented in the previous section were analysed to determine the kinetics of reaction. This was done by modelling rate equations to the experimental data. To minimise contributions from reverse reactions, only data points collected in the first 30 minutes of each sorption test were used. For most data sets this was equivalent to seven data points. The integrated form of the rate equations $1.37-1.38$ were used: For first order kinetics

$$
[\mathbf{M}]=[\mathbf{M}]_{0} \pm[\mathbf{M}]_{\Delta}\left[1-\mathrm{e}^{(-k t)}\right]
$$

and for second order kinetics

$$
[\mathbf{M}]=[\mathbf{M}]_{0} \pm\left([\mathbf{M}]_{\Delta}-\frac{1}{\frac{1}{[\mathbf{M}]_{\Delta}}+k t}\right)
$$

where $[\mathrm{M}]$ is the concentration of the analyte of interest (either $\mathrm{Ca}^{2+}$ or $\mathrm{Cu}^{2+}$ ) and $[\mathrm{M}]_{E q}$, its concentration at equilibrium, is given by $[\mathbf{M}]_{E q}=[\mathbf{M}]_{0} \pm[\mathbf{M}]_{\Delta}$. The negative terms were introduced into the equations when modelling the copper uptake (the concentration at equilibrium is less than the copper concentration at time $t=0$ ); in modelling the calcium release the terms were positive. Figure 3.9 shows the equation modelled to the data. Parameters for these equations are presented in Table 3.2.

$R^{2}$ and $\chi^{2}$ parameters were calculated as follows:

$$
\begin{aligned}
& R^{2}=1-\frac{S S_{E}}{S S_{T}} \\
& \chi^{2}=\frac{w S S_{R}}{N D F}
\end{aligned}
$$




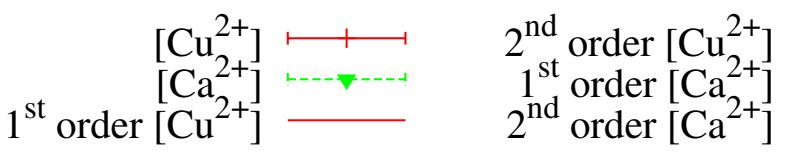

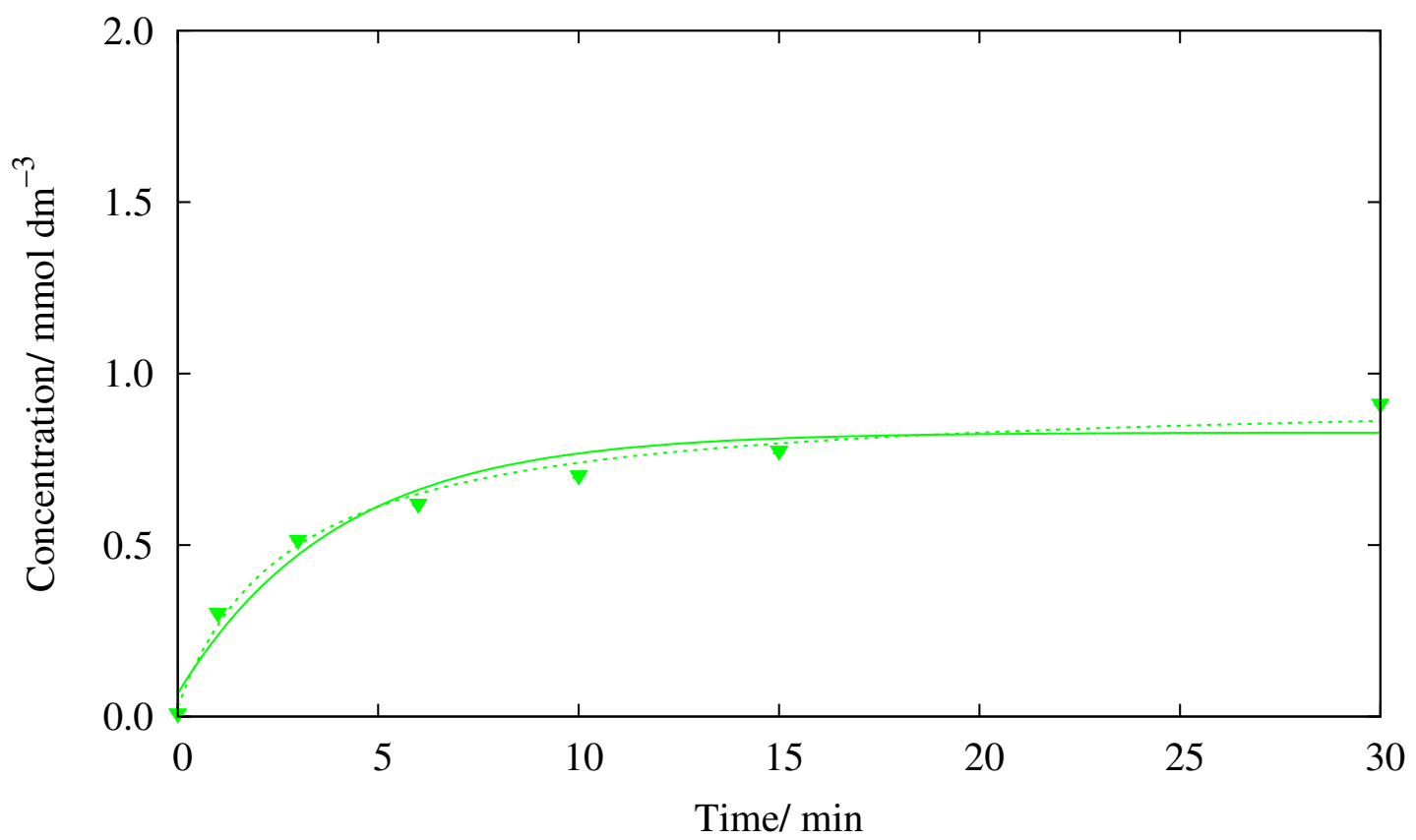

(a)

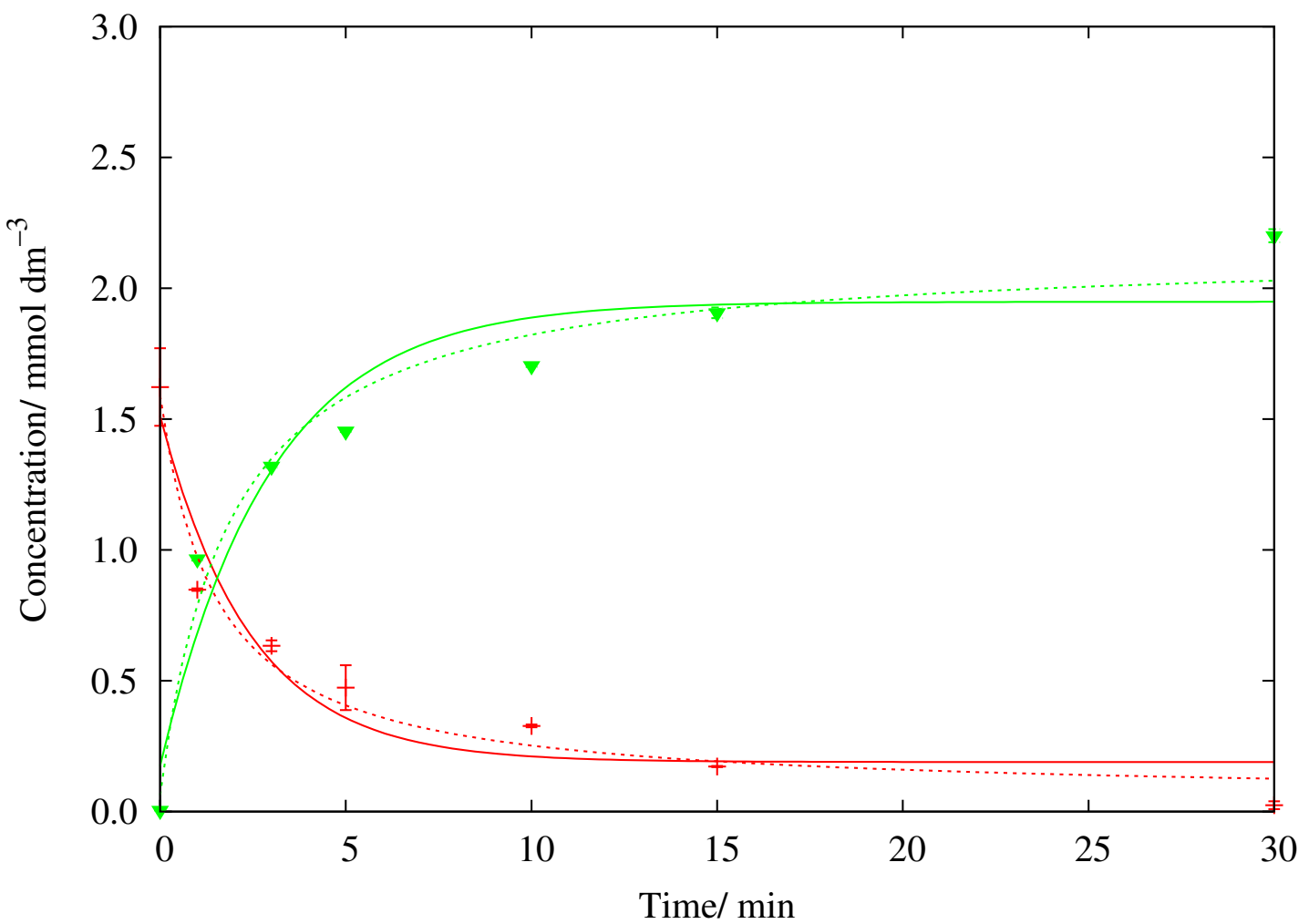

(b)

Figure 3.9 First part of Figure 3.9. (Figure continued over page.) 


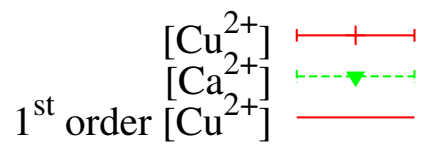
$2^{\text {nd }}$ order $\left[\mathrm{Cu}_{2+}^{2+}\right]$
$2^{\text {nd }}$ order $\left[\mathrm{Ca}^{2+}\right]$

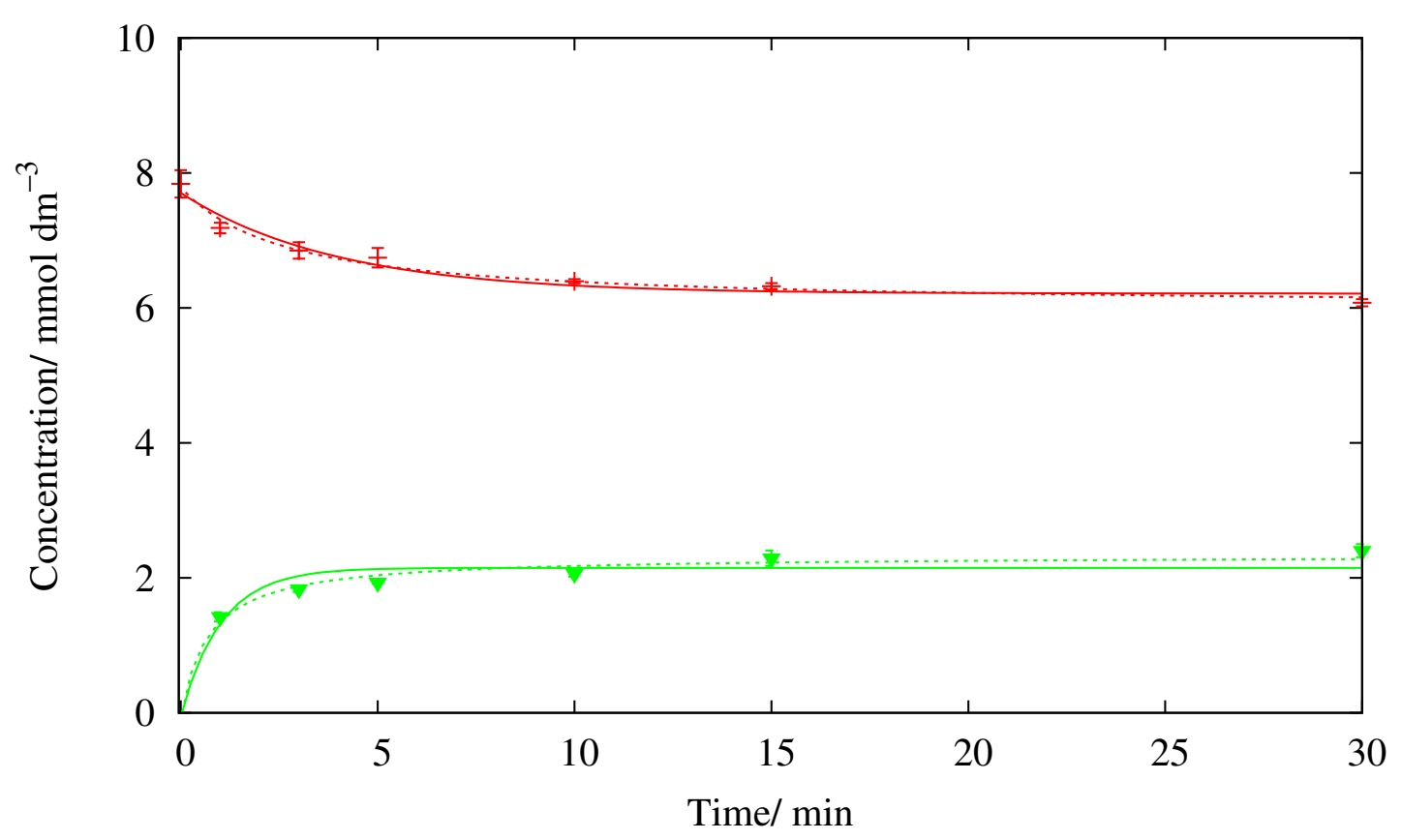

(c)

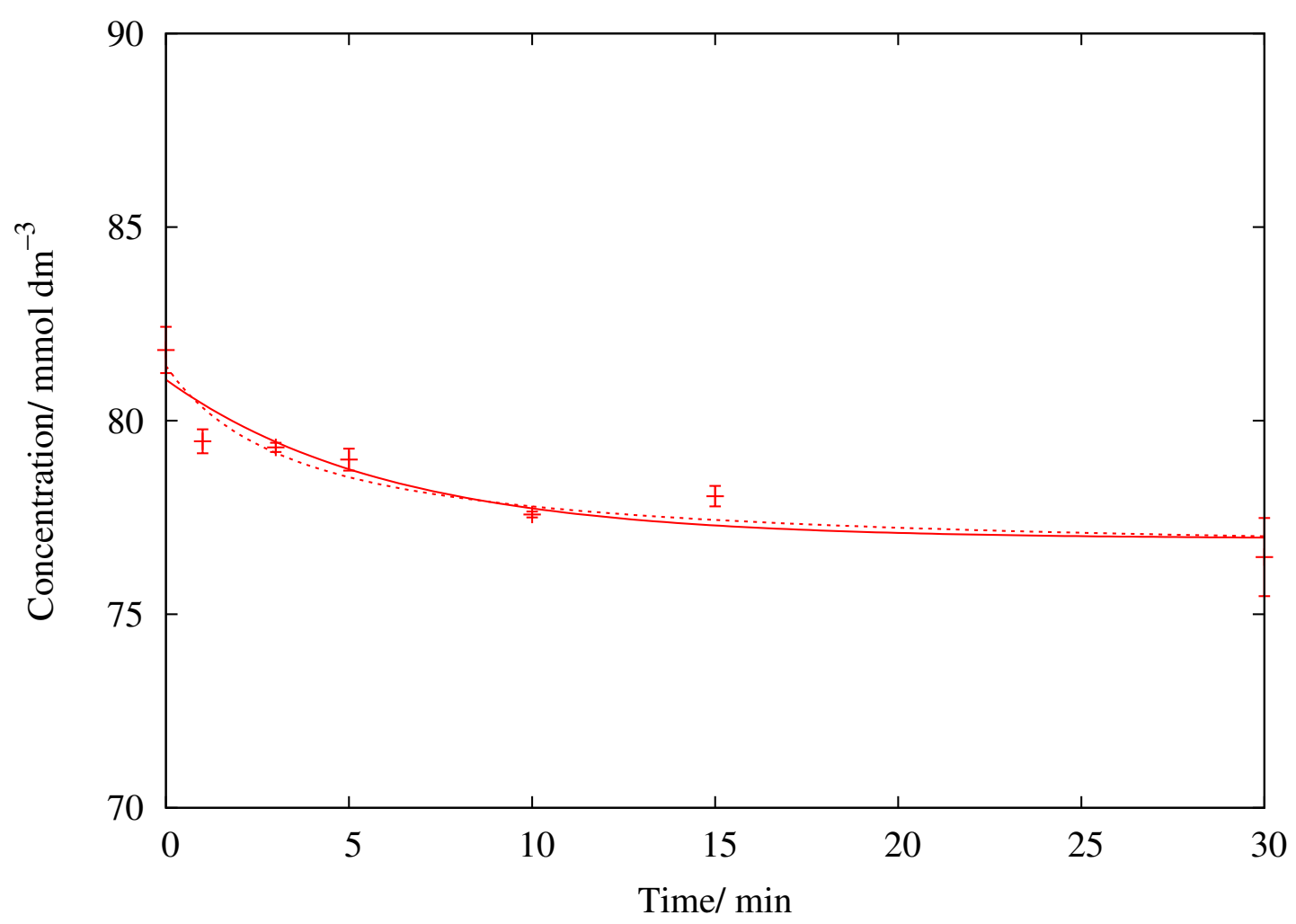

(d)

Figure 3.9 First- and second-order reaction models for sorption of $\mathrm{Cu}^{2+}$ by NCS. Data points in the figures represent the measured $\mathrm{Ca}^{2+}$ or $\mathrm{Cu}^{2+}$ concentrations in

Figures 3.4-3.6. The first order reaction models are shown with solid lines. Dashed lines represent the second order kinetic models: (a) reaction between $\mathrm{NCS}$ and $\mathrm{H}_{2} \mathrm{O}$; (b)-(d) sorption of $\mathrm{Cu}^{2+}$ from a $\mathrm{Cu}\left(\mathrm{NO}_{3}\right)_{2}$ solution. 2-Ethoxyethanol washed NCS applied at a loading of $1 \mathrm{~g} \mathrm{dm}^{-3}$. 
Table 3.2 Rate data for the sorption of $\mathrm{Cu}^{2+}$ from a $\mathrm{Cu}\left(\mathrm{NO}_{3}\right)_{2}$ solution by NCS.

\begin{tabular}{|c|c|c|c|c|}
\hline \multirow{2}{*}{ Parameter } & \multicolumn{4}{|c|}{ Initial $\left[\mathrm{Cu}^{2+}\right] / \mathrm{mmol} \mathrm{dm}^{-3}$} \\
\hline & $\mathbf{0}$ & $1.6 \pm 0.2$ & $7.8 \pm \mathbf{0 . 2}$ & $\mathbf{8 1 . 8} \pm \mathbf{0 . 6}$ \\
\hline \multicolumn{5}{|l|}{ First Order } \\
\hline$k_{\mathrm{Cu}}{ }^{a}$ & N/A & 0.412 & 0.253 & 0.166 \\
\hline$[\mathrm{Cu}]_{0, \text { Calc. }^{b}}$ & N/A & 1.51 & 7.70 & 81.1 \\
\hline$[\mathrm{Cu}]_{\Delta}$ & N/A & 1.32 & 1.49 & 4.11 \\
\hline$R_{\mathrm{Cu}}^{2}$ & N/A & 0.841 & 0.955 & 0.859 \\
\hline$k_{\mathrm{Ca}}$ & 0.253 & 0.338 & 0.974 & 0.965 \\
\hline$[\mathrm{Ca}]_{0, \text { Calc. }}$ & 0.067 & 0.16 & -0.05 & -0.02 \\
\hline$[\mathrm{Ca}]_{\Delta}$ & 0.761 & 1.77 & 2.19 & 2.46 \\
\hline$R_{\mathrm{Ca}}^{2}$ & 0.989 & 0.902 & 0.958 & 0.917 \\
\hline \multicolumn{5}{|c|}{ Second Order } \\
\hline$\overline{k_{\mathrm{Cu}}{ }^{c}}$ & N/A & 0.437 & 0.208 & 0.0567 \\
\hline$[\mathrm{Cu}]_{0, \text { Calc. }}$ & N/A & 1.59 & 7.79 & 81.4 \\
\hline$[\mathrm{Cu}]_{\Delta}$ & N/A & 1.54 & 1.78 & 4.93 \\
\hline$R_{\mathrm{Cu}}^{2}$ & N/A & 0.881 & 0.982 & 0.893 \\
\hline$k_{\mathrm{Ca}}$ & 0.390 & 0.256 & 0.593 & 0.470 \\
\hline$[\mathrm{Ca}]_{0, \text { Calc. }}$ & 0.028 & 0.06 & -0.05 & -0.04 \\
\hline$[\mathrm{Ca}]_{\Delta}$ & 0.912 & 2.09 & 2.40 & 2.72 \\
\hline$R_{\mathrm{Ca}}^{2}$ & 0.997 & 0.946 & 0.988 & 0.963 \\
\hline
\end{tabular}

${ }^{a} 1^{\text {st }}$ order rate constants are presented in dimensions of $\min ^{-1}$.

${ }^{b}$ All concentrations are given in dimensions of $\mathrm{mmol} \mathrm{dm}^{-3}$, and calculated from the rate equation.

${ }^{c} 2^{\text {nd }}$ order rate constants are presented in dimensions of $\mathrm{dm}^{3} \mathrm{mmol}^{-1} \mathrm{~min}^{-1}$. 
where

$$
\begin{aligned}
S S_{T} & =\sum_{i=1}^{n}\left(y_{i}-\bar{y}\right)^{2} \\
S S_{E} & =\sum_{i=1}^{n}\left(y_{i}-f\left(x_{i}\right)\right)^{2} \\
S S_{R} & =\sum_{i=1}^{n}\left(y_{i}-f\left(x_{i}\right)\right)^{2} \\
w S S_{R} & =\sum_{i=1}^{n}\left[\frac{1}{\sigma^{2}}\left(y_{i}-f\left(x_{i}\right)\right)^{2}\right]
\end{aligned}
$$

In the above definitions, $\left(x_{i}, y_{i}\right)$ is an experimental data point, $\bar{y}$, the average of $y, f(x)$ the modelled function and $\sigma^{2}$ the variance with respect to $y . N D F$ represents the degrees of freedom of the model. It is the difference between the number of variables used in the model and the number of data points. The modelling algorithm used minimised $\frac{S S_{R}}{N D F}$, as minimisation of $\chi^{2}$ attributed too great a weighting to data points at low concentration values. $R^{2}$ values were subsequently calculated from the fitted model. $\mathrm{Ng}$ et al. ${ }^{102}$ and Rengaraj et al. ${ }^{103}$, in studies modelling isotherm data have noted that the error function minimised during data modelling can affect calculated $R^{2}$ values, although $R^{2}$ is an error function in its own right.

\subsubsection{Sorption of Copper}

As can be seen from Table 3.2, the kinetic models presented in Equations 3.9 and 3.10 conform moderately well to the data from the uptake of copper. $R_{\mathrm{Cu}}^{2}$ values for the first order process range from 0.841 to 0.955 ; for the second order model, the range is 0.881 to 0.982 . For each individual data set, the second order model exhibited a higher degree of conformity than the first order process. It can also be seen that the rate of the copper uptake process decreases with an increase in the initial copper concentration. For the first order process, the rate constant decreases from $k=0.412 \mathrm{~min}^{-1}$ at an initial $\mathrm{Cu}^{2+}$ concentration of $1.6 \mathrm{mmol} \mathrm{dm}{ }^{-3}$ to a value of $k=0.166 \mathrm{~min}^{-1}$ at an initial $\mathrm{Cu}^{2+}$ concentration of $81.8 \mathrm{mmol} \mathrm{dm}{ }^{-3}$. For the second order process, the rate constant decreases from $k=0.437 \mathrm{dm}^{3} \mathrm{mmol}^{-1} \mathrm{~min}^{-1}$ to $k=0.0567 \mathrm{dm}^{3} \mathrm{mmol}^{-1} \mathrm{~min}^{-1}$ over the same concentration range. If the sorption of copper by NCS could be described by one of 
these kinetic models, the calculated rate constant should be independent of concentration. As this is not the case for sorption of copper by NCS, a simple kinetic model does not adequately describe the system, even though models can be made to conform to the data.

The decrease in the observed rate of copper removal with an increase in copper concentration implies that the system has become rate-limited in some way. Due to the microstructure of NCS, it is highly likely that the degree of accessability of the copper towards the NCS surface varies. This would lead to a change in the $\Delta r$ parameter in the film diffusion mechanism (Equation (1.25)). Some regions of the NCS surface will have a high degree of accessibility, whereas others will be relatively inaccessible. When sorption occurs, it happens preferentially at surface sites with a high accessability. At low initial copper concentrations, these sites are in excess of the amount of copper. As the initial copper concentration is increased, these sites become saturated, and sorption takes place at the less accessible sites. The lower accessibility is accompanied by a slower rate of diffusion (both of $\mathrm{Cu}^{2+}$ from the bulk solution to these sites, and of $\mathrm{Ca}^{2+}$ away from these sites).

As mentioned earlier in Section 1.2.3.1, diffusion through a liquid film, as is occurring at higher copper concentrations, often exhibits similar kinetic behaviour to a simple firstorder reaction model.

\subsubsection{Release of Calcium}

The $R^{2}$ values presented in Table 3.2 show that calcium release conforms more closely to the kinetic models than that of copper sorption. All $R^{2}$ values for calcium are in excess of 0.9 , however they are generally higher for the second-order model. As with copper sorption, the first order rate constants exhibit a dependence on the initial copper concentration. The behaviour of calcium contrasts to that of copper in that calcium is released faster with an increase of the initial copper concentration. This faster release may be due to the lower $\mathrm{pH}$ exhibited by the higher concentration copper solutions. If the $\mathrm{Ca}^{2+}$ initially ion-exchanges with $\mathrm{H}_{3} \mathrm{O}^{+}$, the increased concentration of $\mathrm{H}_{3} \mathrm{O}^{+}$at lower $\mathrm{pH}$ would increase the observed rate. 


\subsubsection{Effect of $\mathrm{pH}$}

To determine the reaction kinetics with respect to $\mathrm{OH}^{-}$, the $\mathrm{pH}$ data obtained from experiments conducted at $\mathrm{Cu}^{2+}$ concentrations of $1.6 \mathrm{mmol} \mathrm{dm}^{-3}$ and below were initially transformed to provide an approximate $\mathrm{OH}^{-}$concentration. ${ }^{\dagger}$ Zeroth-, first- and secondorder kinetic models were applied to the data. For zeroth order kinetics, the following model was applied:

$$
[\mathrm{M}]=[\mathrm{M}]_{0}+k t
$$

This modelling was limited to data sets for which the $\mathrm{pH}$ took more than 30 minutes to equilibrate, in order to provide a sufficient number of data points. The models are shown in Figure 3.10.

The rate of release of $\mathrm{OH}^{-}$by NCS shows no significant difference between the conformity to either a first- or second-order kinetic model. When no copper is present, the system displays good conformity to both models. The $R^{2}$ is slightly higher with the second order model at 0.957 , compared to 0.951 for the first order model. The zeroth order model in this case exhibited a lower degree of conformity at $R^{2}=0.857$. In cases where copper is present, there is no difference in the conformity to either the zeroth-, firstor second- order models, with $R^{2}$ equal to 0.989 in the case of copper chloride and 0.947 in the case of copper nitrate. The fact that the $R^{2}$ value remains unchanged across the various kinetic models implies that the whole reaction is zeroth order; the higher order models may simplify to model a lower order system, but the zeroth order model will never adequately model a system displaying first- or second- order kinetics.

\footnotetext{
$\dagger$ It was assumed that the activity coefficient of $\mathrm{OH}^{-}$in this case was unity, so that the magnitude of $a_{\mathrm{OH}^{-}}$ was identical to that of $\left[\mathrm{OH}^{-}\right]$. The relationship between $\mathrm{pH}$ and $\mathrm{pOH}$ was taken as $\mathrm{pH}+\mathrm{pOH}=14$.
} 

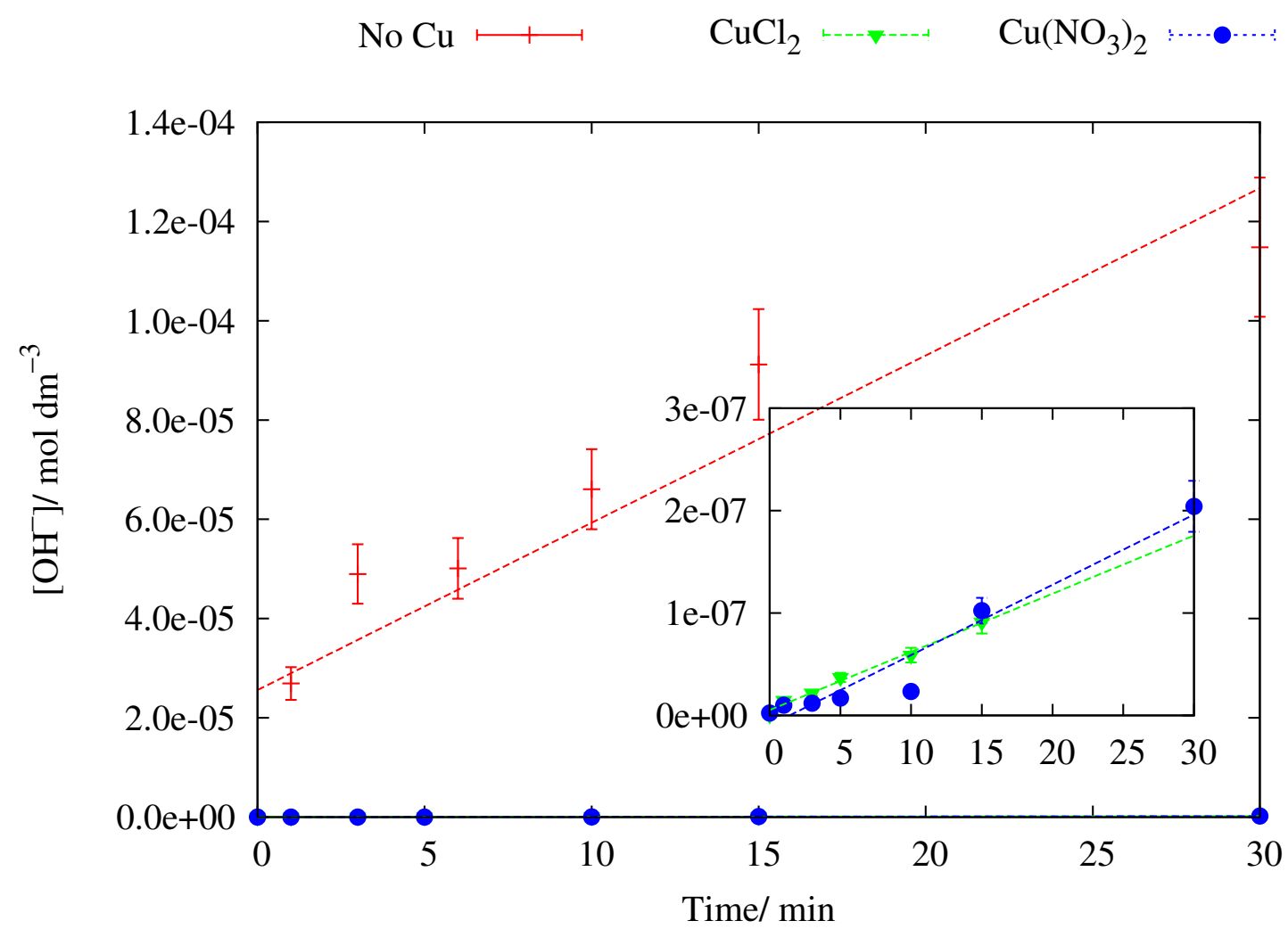

(a)

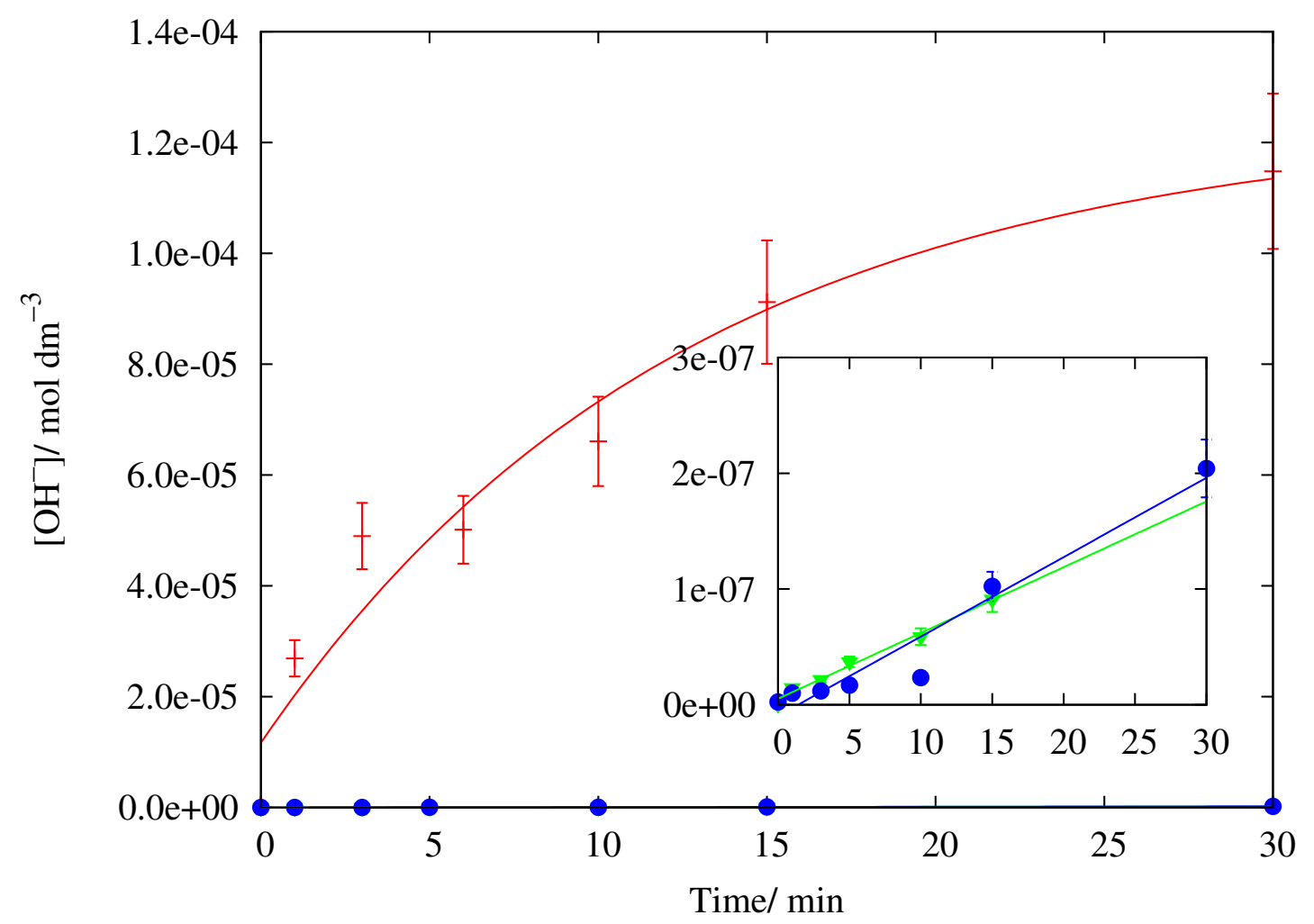

(b)

Figure 3.10 First part of Figure 3.10. (Figure continued over page.) 
Table 3.3 Rate data for $\mathrm{OH}^{-}$release by NCS during sorption of $\mathrm{Cu}^{2+}$.

\begin{tabular}{llll}
\hline \multirow{2}{*}{ Parameter } & \multicolumn{4}{c}{$\mathrm{Cu}^{2+}$ Source, } & $1.6 \mathrm{mmol} \mathrm{dm}^{-3}$ \\
\cline { 2 - 4 } & $\mathrm{N} / \mathrm{A}$ & $\mathrm{CuCl}_{2}$ & $\mathrm{Cu}\left(\mathrm{NO}_{3}\right)_{2}$ \\
\hline
\end{tabular}

\section{Zeroth Order}

\begin{tabular}{lrrr}
\hline$k_{\mathrm{OH}^{-}}{ }^{a}$ & $3.37 \times 10^{-6}$ & $5.68 \times 10^{-8}$ & $6.86 \times 10^{-9}$ \\
{$\left[\mathrm{OH}^{-}\right]_{0, \text { Calc. }}{ }^{b}$} & $2.56 \times 10^{-5}$ & $5.20 \times 10^{-9}$ & $-9.68 \times 10^{-9}$ \\
$R_{\mathrm{OH}^{-}}^{2}$ & 0.857 & 0.989 & 0.947
\end{tabular}

\section{First Order}

\begin{tabular}{lrrrr}
\cline { 1 - 2 }$k_{\mathrm{OH}^{-}}{ }^{c}$ & & $7.97 \times 10^{-2}$ & $2.84 \times 10^{-8}$ & $1.14 \times 10^{-7}$ \\
{$\left[\mathrm{OH}^{-}\right]_{0, \text { Calc. }}$} & $1.17 \times 10^{-5}$ & $2.50 \times 10^{-8}$ & $-9.68 \times 10^{-9}$ \\
{$\left[\mathrm{OH}^{-}\right]_{\Delta}$} & & $1.12 \times 10^{-4}$ & 0.200 & $6.00 \times 10^{-2}$ \\
$R_{\mathrm{OH}^{-}}^{2}$ & 0.951 & 0.989 & 0.947 \\
\cline { 1 - 2 } Second Order & & & \\
${^{-}}{ }^{2} } }$ & $5.00 \times 10^{2}$ & $1.42 \times 10^{-7}$ & $1.25 \times 10^{-6}$ \\
{$\left[\mathrm{OH}^{-}\right]_{0, \text { Calc. }}$} & $9.52 \times 10^{-6}$ & $5.20 \times 10^{-9}$ & $-9.68 \times 10^{-9}$ \\
{$\left[\mathrm{OH}^{-}\right]_{\Delta}$} & $1.50 \times 10^{-4}$ & 0.200 & $7.41 \times 10^{-2}$ \\
$R_{\mathrm{OH}^{-}}^{2}$ & 0.957 & 0.989 & 0.947 \\
\hline
\end{tabular}

${ }^{a} 0^{\text {th }}$ order rate constants are presented in dimensions of $\mathrm{mol} \mathrm{dm}^{-3} \mathrm{~min}^{-1}$.

${ }^{b}$ All concentrations are given in dimensions of $\mathrm{mol} \mathrm{dm}^{-3}$, and calculated from the rate equation.

${ }^{c} 1^{\text {st }}$ order rate constants are presented in dimensions of $\min ^{-1}$.

$d_{2}{ }^{\text {nd }}$ order rate constants are presented in dimensions of $\mathrm{dm}^{3} \mathrm{~mol}^{-1} \mathrm{~min}^{-1}$. 


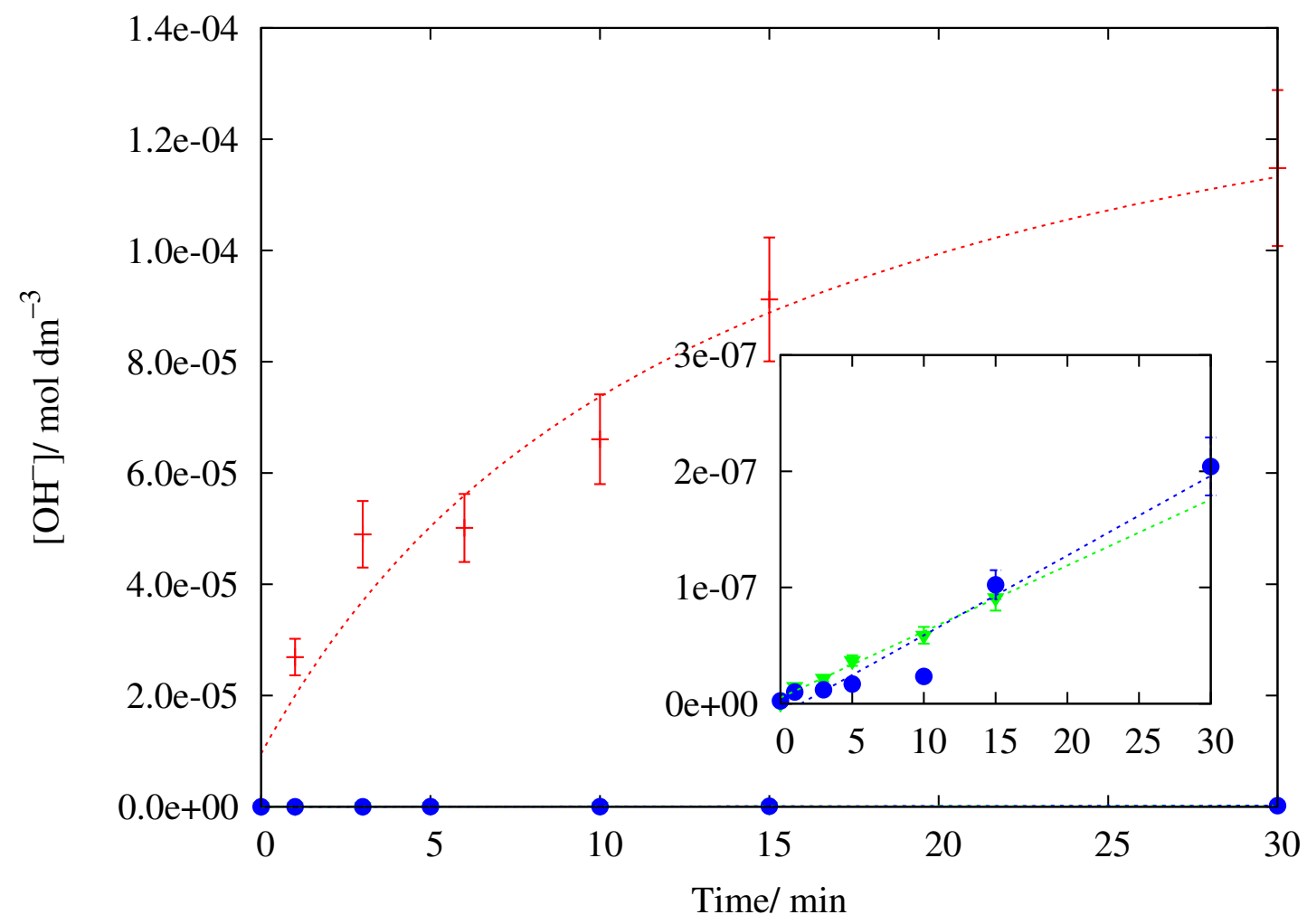

(c)

Figure 3.10 Kinetics of hydrogen ion uptake by NCS in the presence of $1.6 \mathrm{mmol} \mathrm{dm}{ }^{-3}$ $\mathrm{Cu}^{2+}$ : (a) zeroth order kinetics; (b) first order kinetics; (c) second order kinetics.

\subsubsection{Surface Analysis}

\subsubsection{X-Ray Diffraction}

Figure 3.11 shows the XRD pattern for the spent NCS material following sorption of copper (as $\left.\mathrm{Cu}\left(\mathrm{NO}_{3}\right)_{2}\right)$. At low initial concentrations of copper, only low intensity diffractions are observed as the signal to noise ratio is very low. In many cases, a broad peak at a d-spacing of approximately $2-4 \AA\left(30^{\circ} 2 \theta\right)$ is present. The broad nature of this peak is indicative of the amorphous character of the material. Nonetheless, some crystalline peaks are still observed in these diffraction patterns. With the exception of the intense narrow peak occasionally observed at a d-spacing of $1.43 \AA\left(65^{\circ} 2 \theta\right)$, which can be attributed to the aluminium sample holder used during XRD acquisition, the remaining peaks are mostly characteristic of NCS itself. Due to their broad nature however, their assignment is ambiguous. Previously it has been suggested by McFarlane ${ }^{22}$ that diffraction peaks are due to calcium carbonate impurities and short-range order present in calcium silicate hydrates (particularly the peak at a d-spacing of approximately $3 \AA$ 
$\left(30^{\circ} 2 \theta\right)$, described by Chen et al. $\left.{ }^{23}\right)$.
$0 \mathrm{mmol} \mathrm{dm} \mathrm{m}^{-3}$
$1.6 \mathrm{mmol} \mathrm{dm}^{-3}$
$7.8 \mathrm{mmol} \mathrm{dm}^{-3}$
$82 \mathrm{mmol} \mathrm{dm}^{-3}$

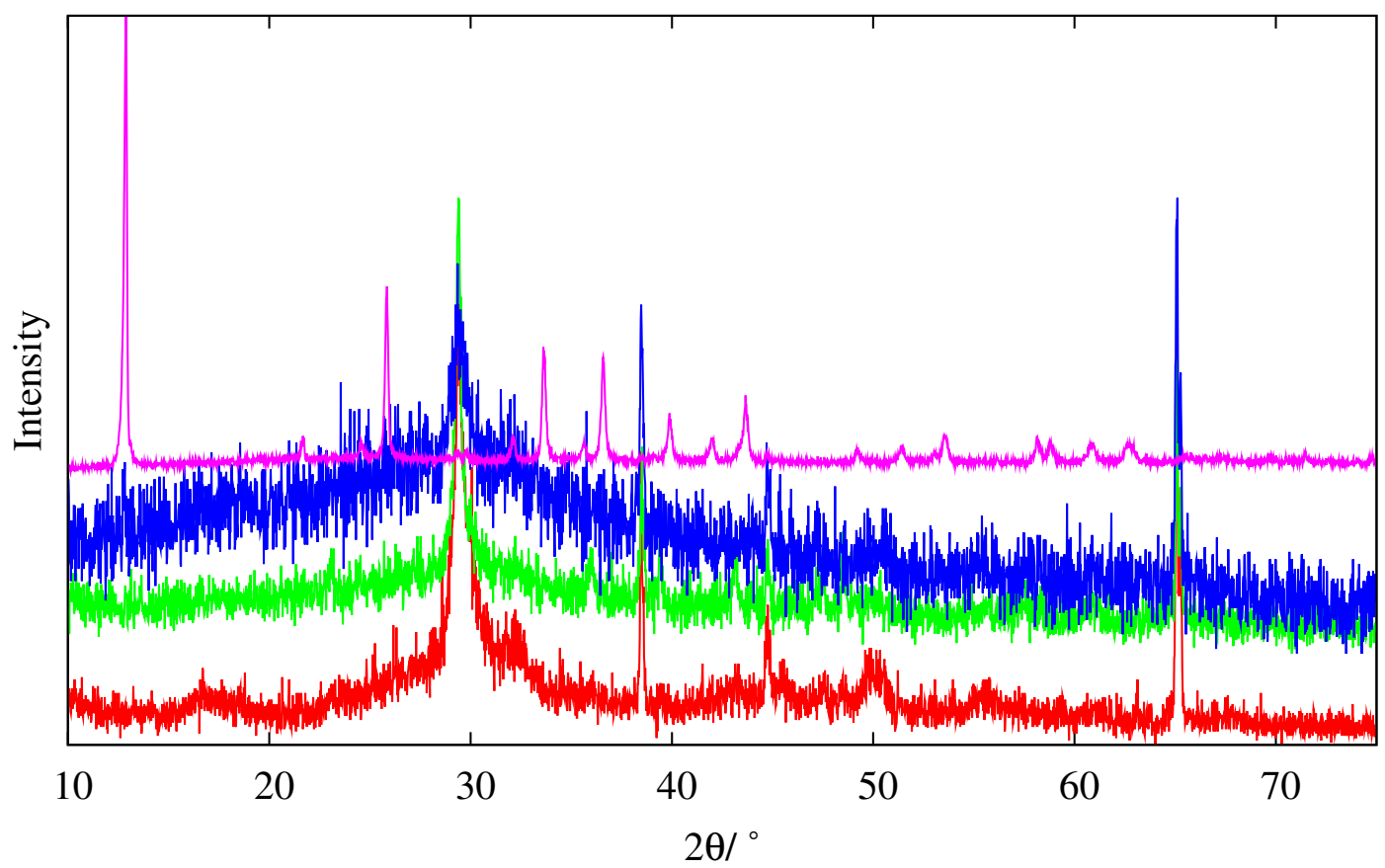

Figure 3.11 Powder X-ray diffraction patterns of spent NCS material following sorption at various initial concentrations of $\mathrm{Cu}\left(\mathrm{NO}_{3}\right)_{2}$.

After saturation of the NCS with copper nitrate, a crystalline phase is easily observed in the diffraction pattern. This diffraction pattern may be assigned to the mineral rouaite, $\mathrm{Cu}_{2}\left(\mathrm{NO}_{3}\right)(\mathrm{OH})_{3}$ (PDF pattern 00-045-0594). ${ }^{41}$ The diffraction pattern exhibited by the NCS material following copper chloride sorption is slightly more complex. Three different minerals have the analogous chemical formula $\left(\mathrm{Cu}_{2} \mathrm{Cl}(\mathrm{OH})_{3}\right)$ : atacamite (PDF 00-025-0269), ${ }^{41}$ clinoatacamite (PDF 00-050-1559) ${ }^{41}$ and paratacamite (PDF 01-0700821). ${ }^{41}$ Atacamite has an orthorhombic space group, clinoatacamite is monoclinic and paratacamite is rhombohedral. The ten most intense diffraction peaks for each mineral are shown in Figure 3.12, along with the experimental diffraction pattern. The most intense diffraction peak is common to all three atacamite minerals and the experimental pattern, at a d-spacing of 5.45-5.47 $\AA\left(16^{\circ} 2 \theta\right)$. In the experimental pattern, the broad peak centred at a d-spacing of $5.04 \AA\left(17.5^{\circ} 2 \theta, 39 \%\right.$ intensity) is present in only the atacamite reference pattern. If the atacamite diffraction pattern is scaled by $56 \%$, the $5.04 \AA$ A peak may be fully attributed to this reference pattern. By scaling the clinoatacamite and paratacamite patterns to a combined total of $44 \%$, the remainder of the experimental 
pattern may be accounted for.

$\begin{array}{rrr}\mathrm{CuCl}_{2} & \begin{array}{r}\text { Clinoatacamite } \\ \text { Paratacamite }\end{array} \\ \mathrm{Cu}\left(\mathrm{NO}_{3}\right)_{2} & * & * \\ \text { Atacamite } & * & * \\ \text { Rouaite, Synthetic }\end{array}$

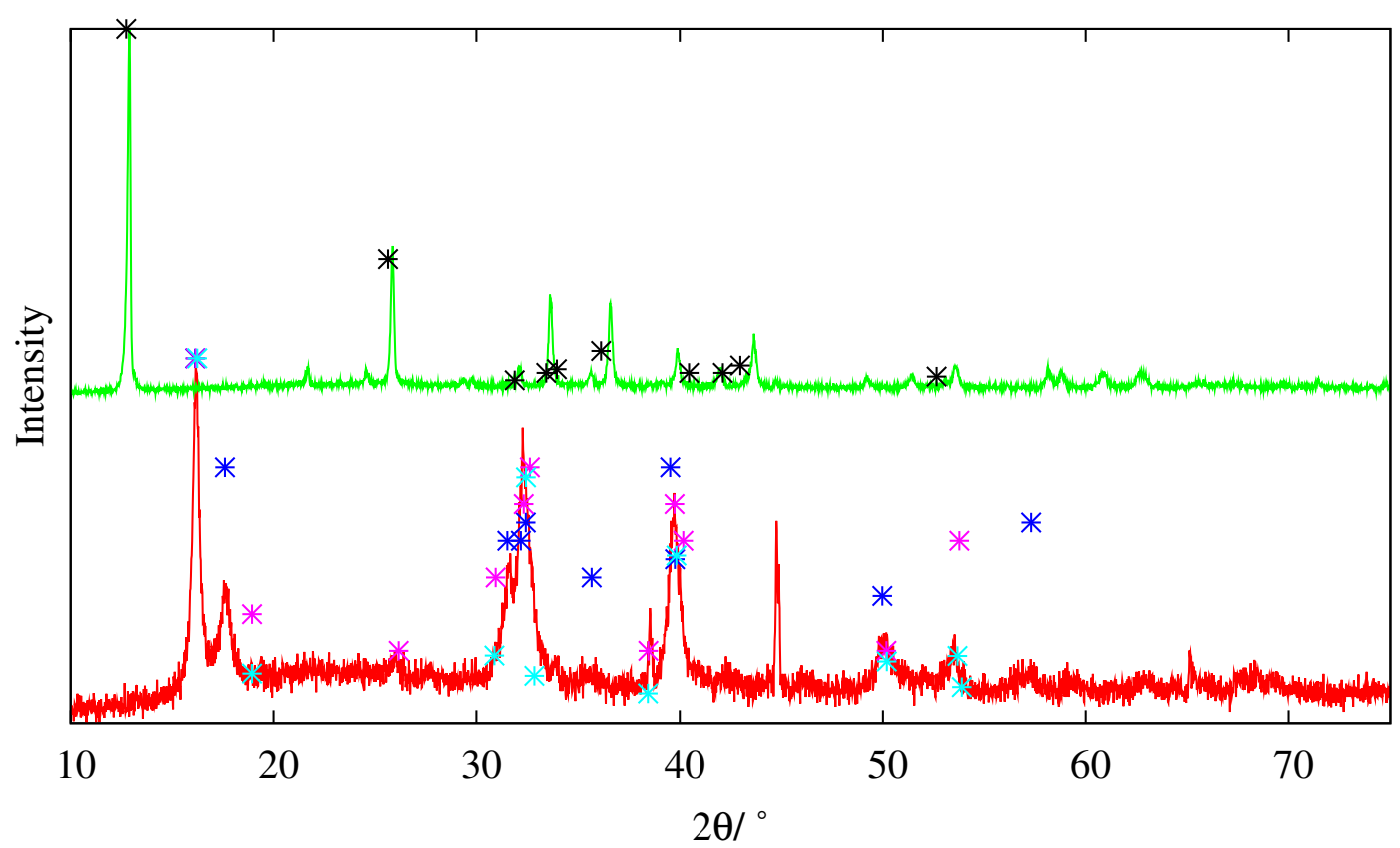

Figure 3.12 Powder X-ray diffraction patterns of spent NCS material following sorption

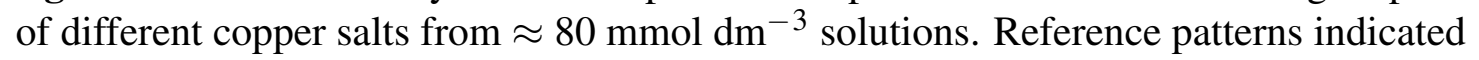
with asterisks.

It has previously been demonstrated by Näsänen and Tamminen ${ }^{51}$ that $\mathrm{Cu}_{2} \mathrm{X}(\mathrm{OH})_{3}$ phases are thermodynamically stable precipitates with respect to $\mathrm{Cu}(\mathrm{OH})_{2}$ in cases where the mole ratio of $\mathrm{OH}^{-}: \mathrm{Cu}^{2+}$ is less than $0.5-1: 1$ (depending on the ionic strength of the solution). $\mathrm{NCS}$, at $28 \mathrm{wt} \% \mathrm{Ca}^{2+}$, contains approximately $7.1 \mathrm{mmol} \mathrm{Ca} \mathrm{Ca}^{2+} \mathrm{g}^{-1}$. The calcium may ion exchange for $\mathrm{H}^{+}$, in effect releasing $\mathrm{OH}^{-}$at twice the rate of the corresponding $\mathrm{Ca}^{2+}$ release resulting in a theoretical maximum hydroxide concentration of $14.2 \mathrm{mmol} \mathrm{dm}^{-3}$, when NCS is applied at a loading of $1 \mathrm{~g} \mathrm{dm}^{-3}$. Thus in cases where the initial copper concentration was approximately $80 \mathrm{mmol} \mathrm{dm}^{-3}$, $\mathrm{Cu}_{2} \mathrm{X}(\mathrm{OH})_{3}$ phases are preferred. When $\mathrm{Cu}^{2+}$ is present at a lower initial concentration $\left(\left[\mathrm{Cu}^{2+}\right] \leq 8 \mathrm{mmol} \mathrm{dm}^{-3}\right), \mathrm{Cu}(\mathrm{OH})_{2}$ is the thermodynamically stable precipitate. It has been observed by Spark et al. ${ }^{104}$ that $\mathrm{Cu}(\mathrm{OH})_{2}$ is an intermediate in the formation of $\mathrm{Cu}_{2} \mathrm{Cl}(\mathrm{OH})_{3}$ and $\mathrm{Cu}_{2}\left(\mathrm{NO}_{3}\right)(\mathrm{OH})_{3}$. The authors noted the X-ray amorphous nature of the initial precipitates formed upon addition of $\mathrm{OH}^{-}$to a solution of $\mathrm{CuCl}_{2}$ or $\mathrm{Cu}\left(\mathrm{NO}_{3}\right)_{2}$, and the lack of $\mathrm{Cl}^{-}$or $\mathrm{NO}_{3}^{-}$in the precipitates. 
The formation of $\mathrm{Cu}(\mathrm{OH})_{2}$ at low initial copper concentrations serves to explain the $\mathrm{X}$ ray amorphous nature of the spent NCS materials following sorption of copper from low concentration solutions.

The diffraction patterns obtained from the copper chloride sorbed NCS display a lower signal to noise ratio than those obtained from the copper nitrate sorption, even though the total amount of copper sorbed was similar for both the chloride and nitrate salts. This lower signal to noise ratio is indicative of a less crystalline material in the case of the copper chloride system. Electron microscopy subsequently demonstrated this to be the case.

\subsubsection{Electron Microscopy}

Figure 3.13 shows electron micrographs of spent NCS material following sorption of copper from an $80 \mathrm{mmol} \mathrm{dm}{ }^{-3} \mathrm{Cu}\left(\mathrm{NO}_{3}\right)_{2}$ solution. Plate-like crystals of a few micrometres in size can be seen in the figure. Although the space group of the crystals cannot be unambiguously assigned based on the images, the plate-like shape of the crystals is consistent with a monoclinic space group, although a triclinic space group is another possibility. Rouaite, the crystalline phase identified through X-ray diffraction studies, has a monoclinic space group. Virtually no pristine NCS, with the characteristic gypsum desert rose morphology, can be observed within the samples originating from 2-ethoxyethanol washed NCS (Figures 3.13(a)-3.13(c)). In the pH adjusted NCS sample however (Figure 3.13(d)), NCS and rouaite can be observed in close proximity. This indicates that the NCS acts as a nucleation site for the growth of the crystalline copper phase.

By contrast copper nitrate, Figure 3.14 shows electron micrographs taken of the spent NCS material following sorption of copper from an $80 \mathrm{mmol} \mathrm{dm}^{-3} \mathrm{CuCl}_{2}$ solution. It can be seen in this figure that the spent NCS still retains the gypsum desert rose morphology of the pristine material. At magnification factors of greater than 10000 times, small crystallites can be observed within the pores of the NCS. At the 10000 and 20000 times magnification, they can be seen as bright spots against the darker silicate 


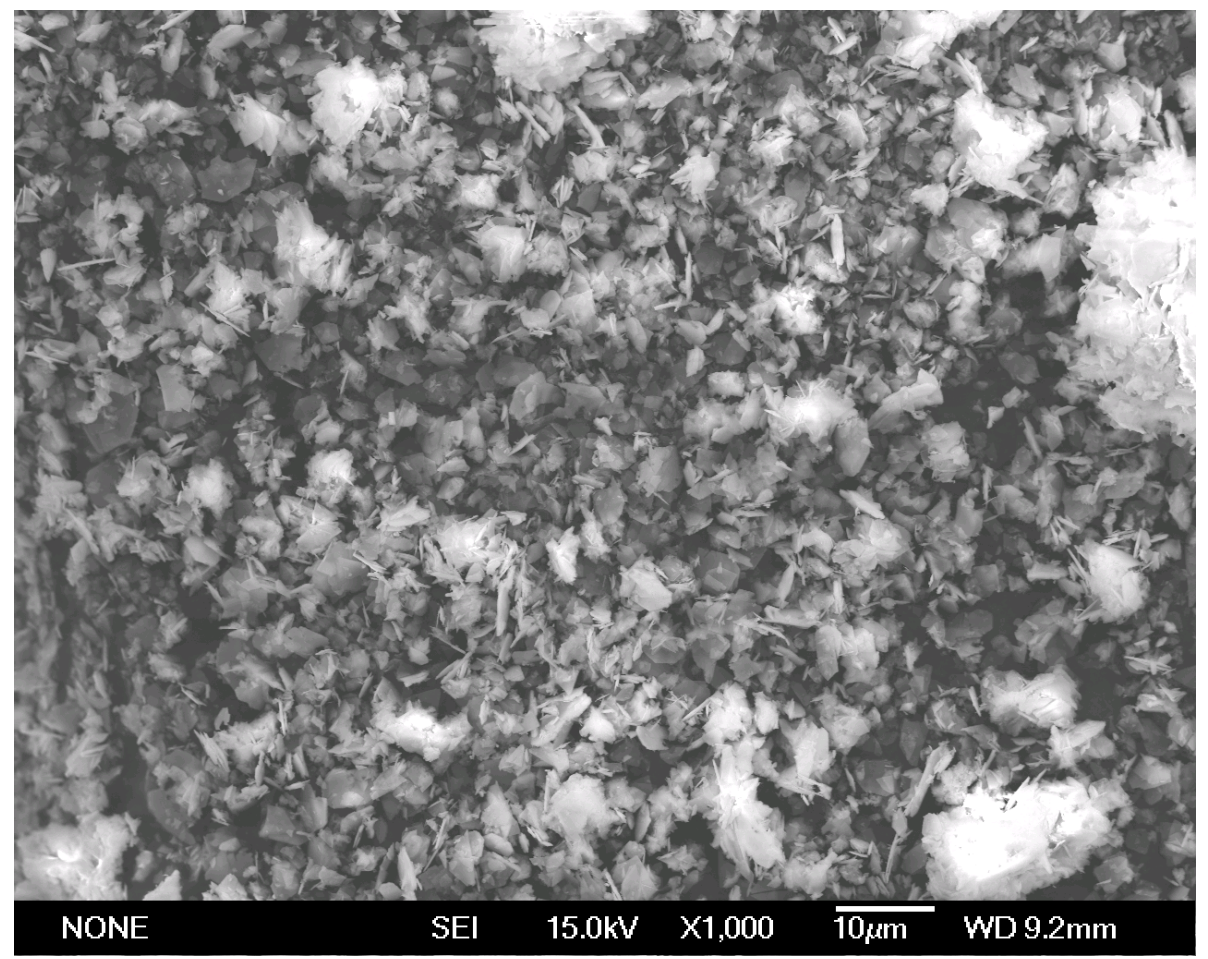

(a)

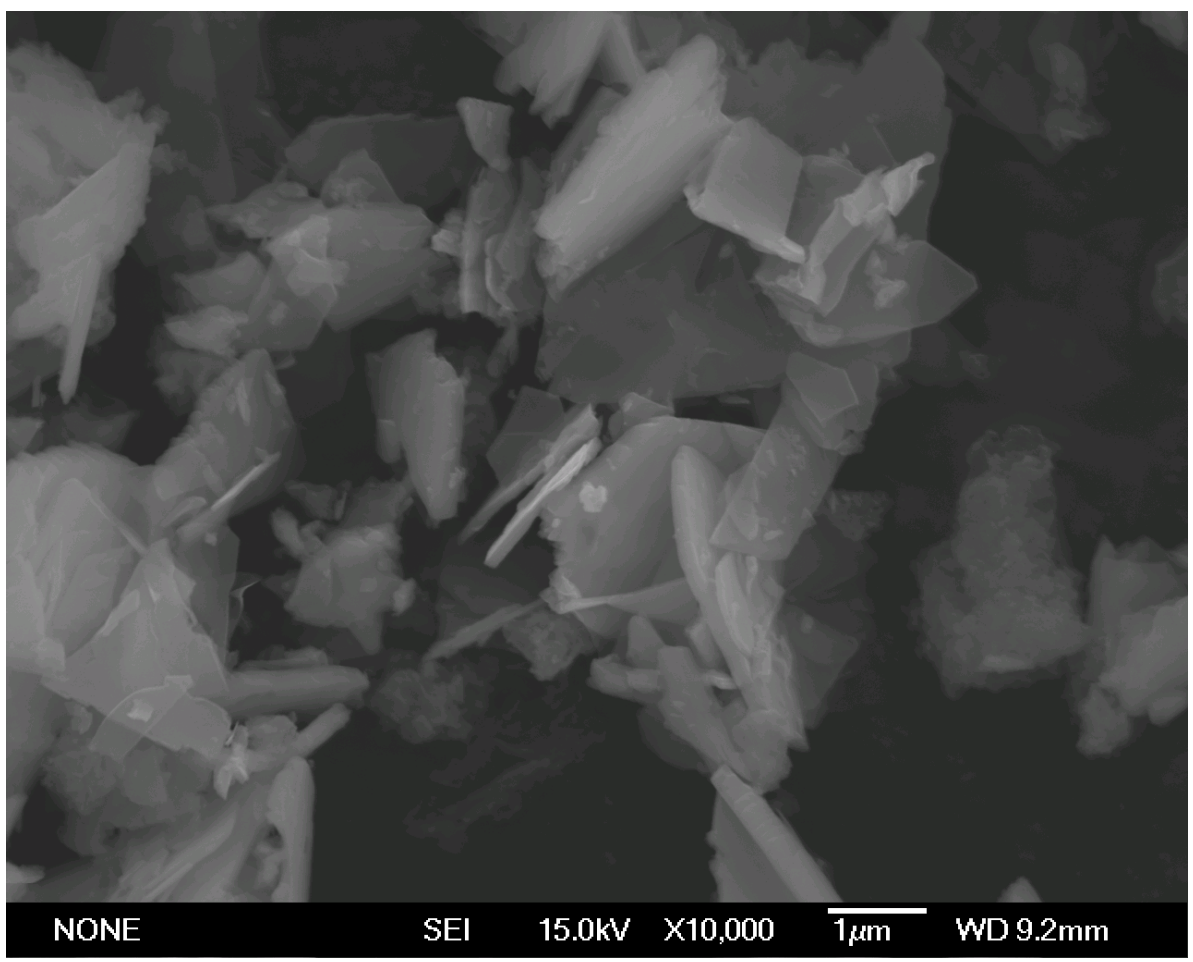

(b)

Figure 3.13 First part of Figure 3.13. (Figure continued over page.) 


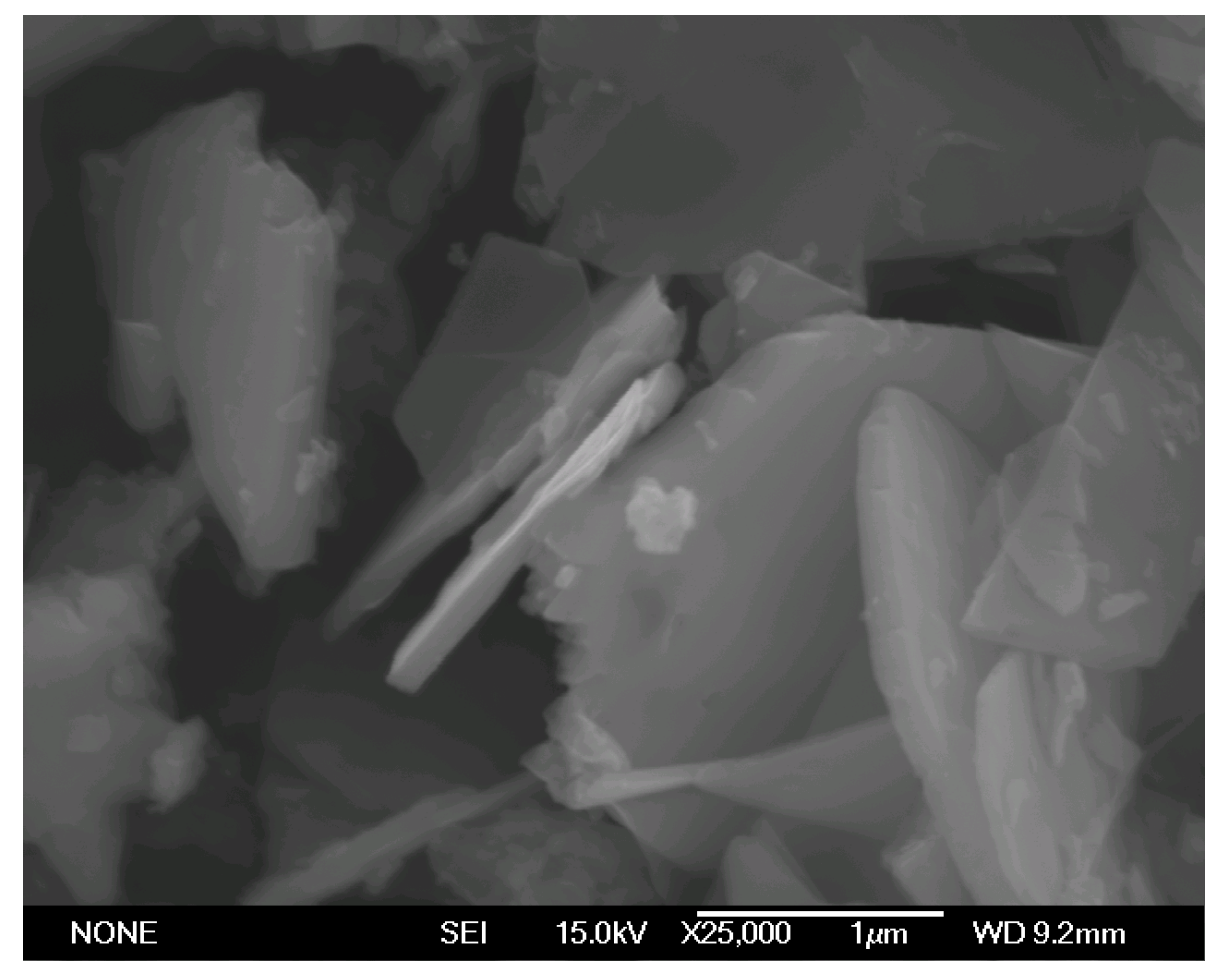

(c)

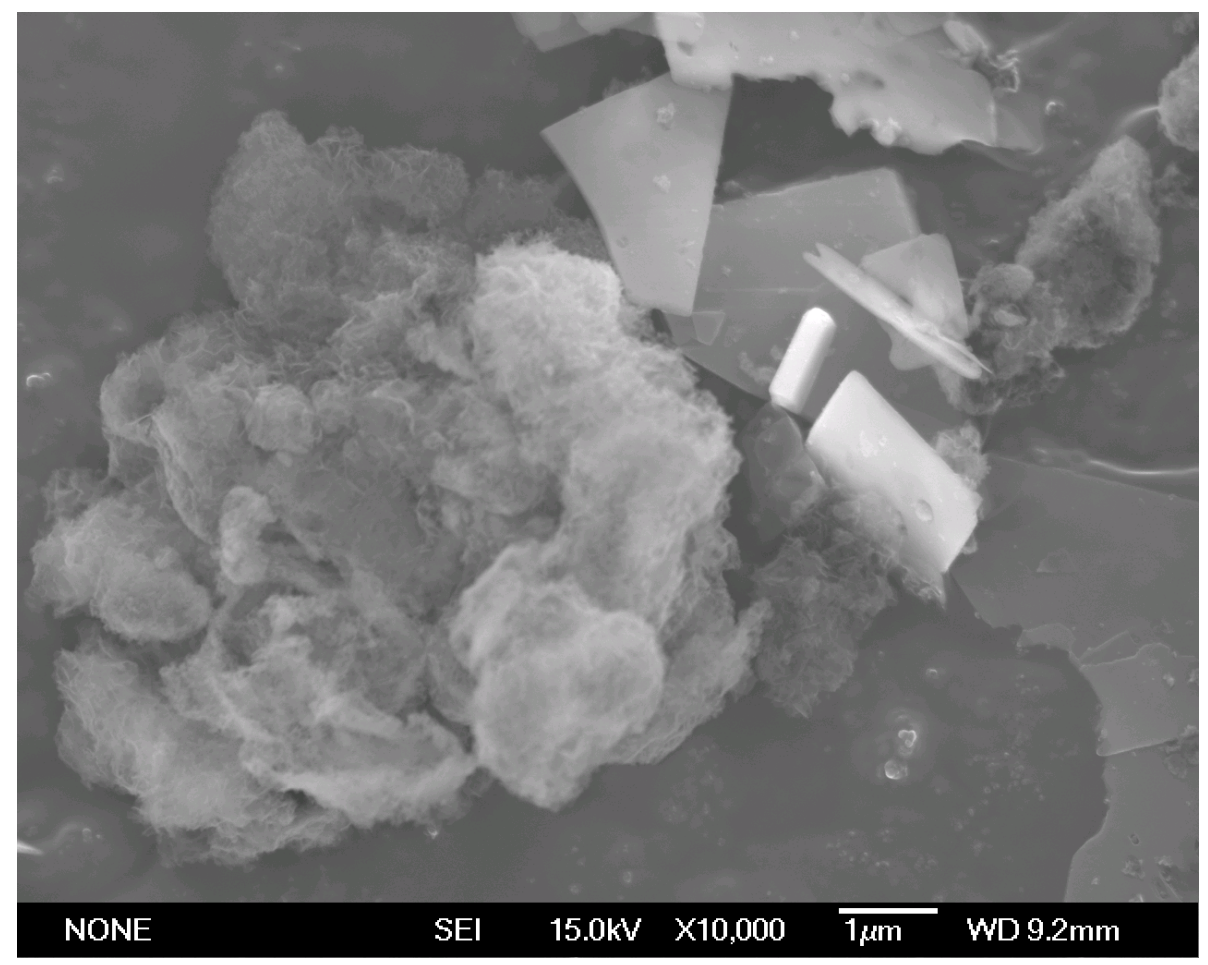

(d)

Figure 3.13 Electron micrographs of spent NCS following sorption of copper nitrate: (a)-(c) sorption by 2-ethoxyethanol washed NCS; (d) sorption by $\mathrm{pH}$ adjusted NCS. NCS applied at a loading of $1 \mathrm{~g} \mathrm{dm}^{-3}$ to $80 \mathrm{mmol} \mathrm{dm}^{-3} \mathrm{Cu}\left(\mathrm{NO}_{3}\right)_{2}$. 
background. At the 50000 times magnification factor, the NCS sample begins to exhibit charging effects, which reduces the contrast and causes artifacts in the image. However it is still possible to estimate the size of the crystallites, from the high magnification image, as 50-100 nm. This indicates that the crystallites are 20-40 times smaller than the $\mathrm{Cu}_{2}\left(\mathrm{NO}_{3}\right)(\mathrm{OH})_{3}$ crystals observed in Figure 3.13, which accounts for the lower signal to noise ratio observed in the X-ray diffraction pattern for the copper chloride sorbed NCS.
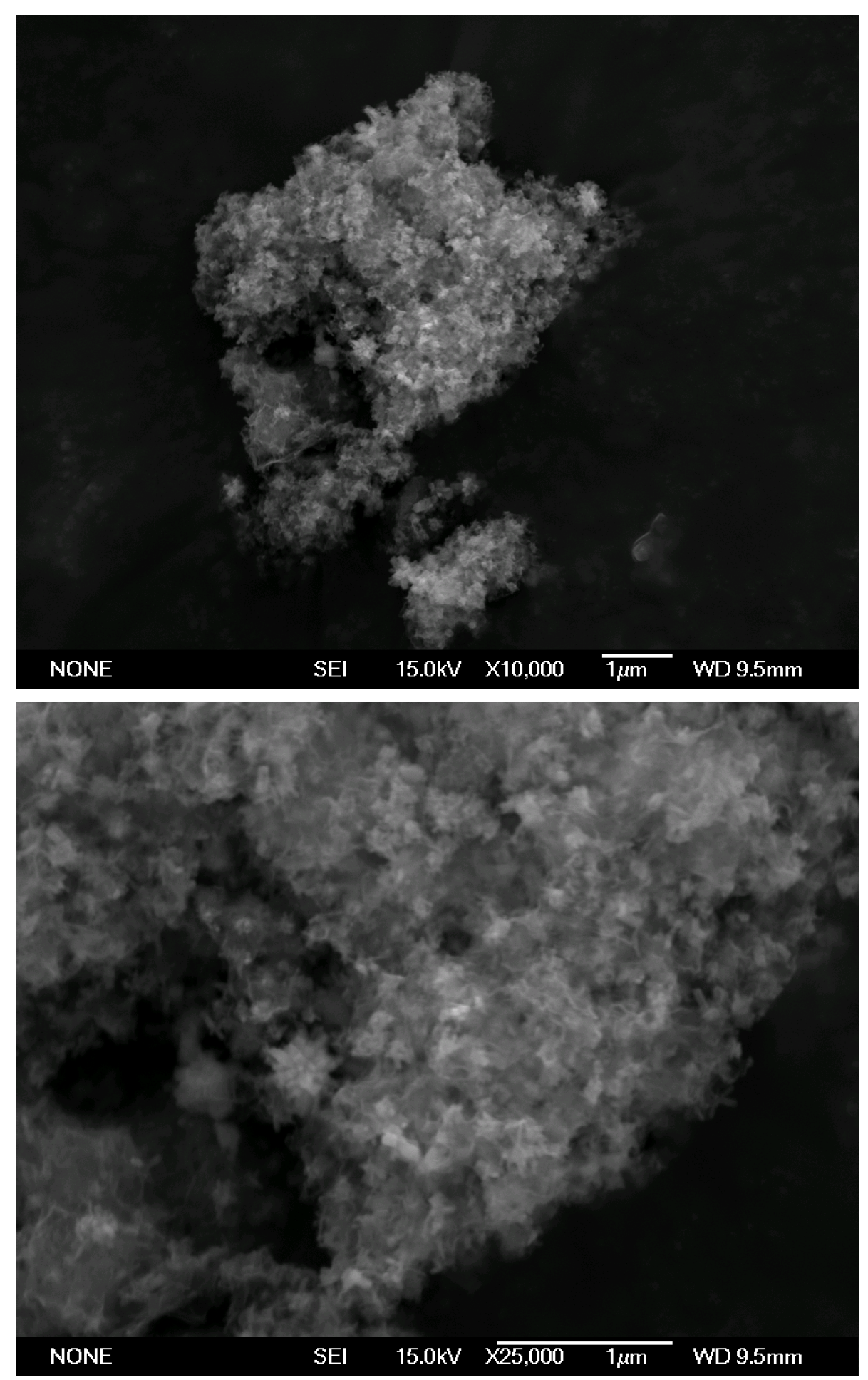

Figure 3.14 First part of Figure 3.14. (Figure continued over page.) 


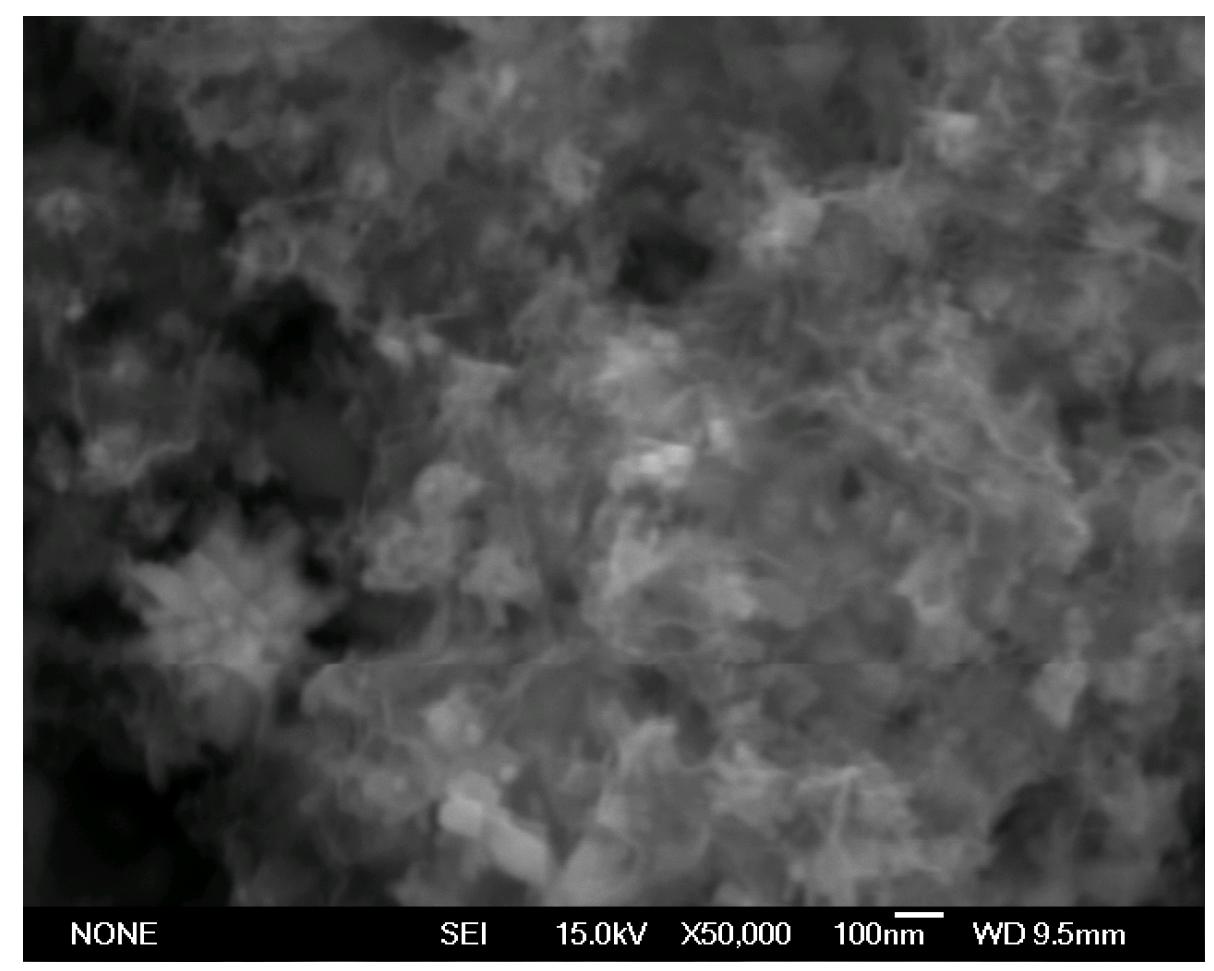

Figure 3.14 Electron micrographs of spent NCS following sorption of copper from a copper chloride solution.

When sorption studies were conducted at low copper concentrations, the spent sorbent exhibited a similar morphology to of pristine NCS. As can be seen in Figure 3.15, platelike crystalline phases, indicative of $\mathrm{Cu}_{2}\left(\mathrm{NO}_{3}\right)(\mathrm{OH})_{2}$, are not present. As will be shown later, copper is still detected in these samples, through the use of EDS. 


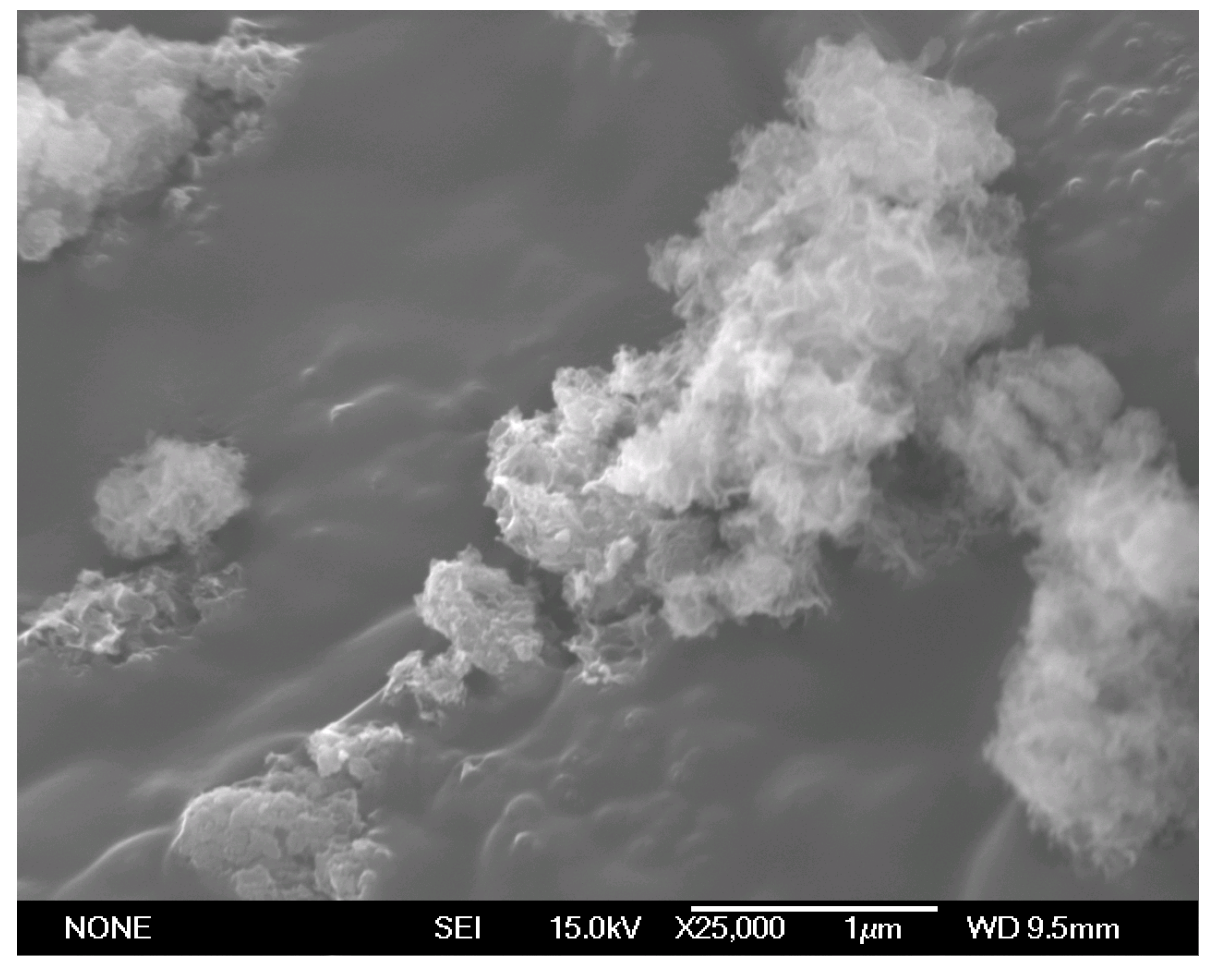

(a)

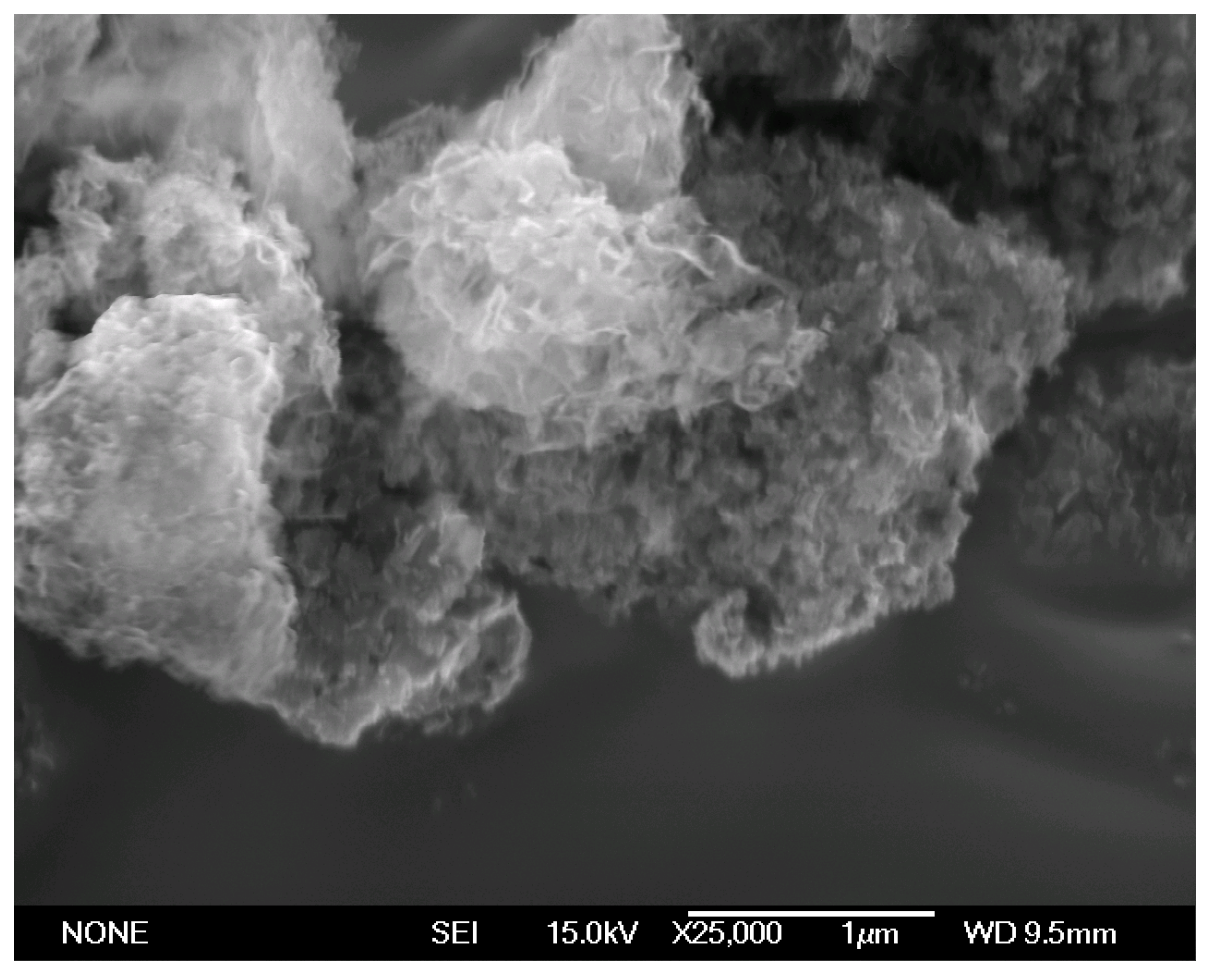

(b)

Figure 3.15 First part of Figure 3.15. (Figure continued over page.) 


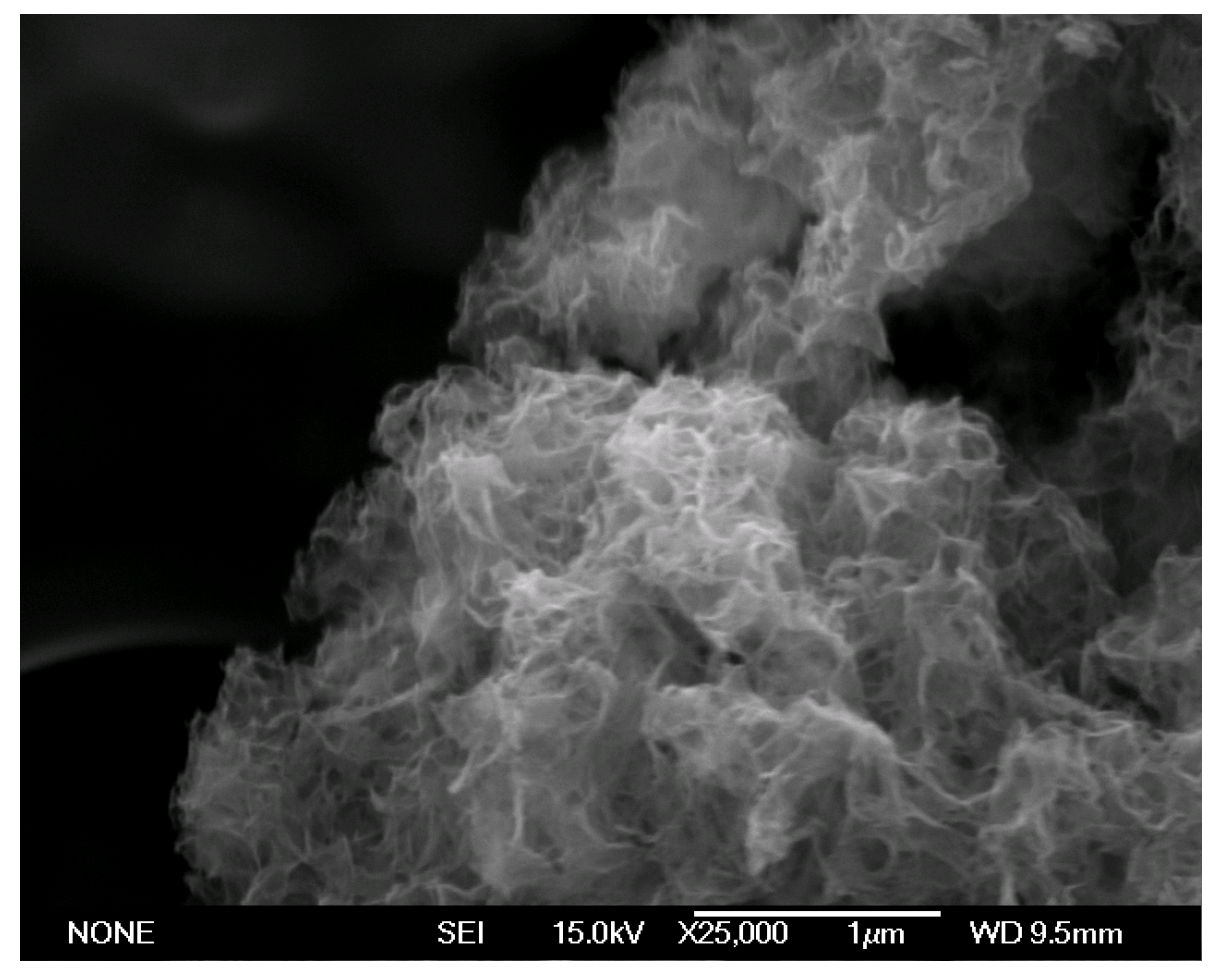

(c)

Figure 3.15 Electron micrographs of spent NCS following sorption of $\mathrm{Cu}^{2+}$ from a $\mathrm{Cu}\left(\mathrm{NO}_{3}\right)_{2}$ solution at $1.6 \mathrm{mmol} \mathrm{dm}{ }^{-3}$ : (a) 2-ethoxyethanol washed $\mathrm{NCS}$; (b) $\mathrm{pH}$ adjusted NCS; (c) 2-ethoxyethanol washed NCS $1.6 \mathrm{mmol} \mathrm{dm}{ }^{-3} \mathrm{CuCl}_{2}$, $0.4 \mathrm{mmol} \mathrm{dm}^{-3} \mathrm{I}_{2}$. 


\subsubsection{Energy Dispersive Spectroscopy}

Figure 3.16 shows an overlay EDS image of NCS following sorption of $\mathrm{Cu}^{2+}$ from an $80 \mathrm{mmol} \mathrm{dm}{ }^{-3} \mathrm{Cu}\left(\mathrm{NO}_{3}\right)_{2}$ solution. The EDS was measured at the same magnification as the electron micrograph shown in Figure 3.13(a), namely 1000 times. It can be seen from the overlay image that the crystalline materials identified in Figure 3.13(a) contain copper (mapped as a green colour), but a relatively small amount of silicon (red). In some regions of the image, silicon is present without copper. As the silicon is indicative of NCS, this indicates that the crystalline copper material and NCS can exist separately. This separation is probably due to the large size of the crystals when compared to the NCS particles. If the NCS acts as the initial nucleation site for $\mathrm{Cu}_{2}\left(\mathrm{NO}_{3}\right)(\mathrm{OH})_{3}$ growth, increasing the size of the crystal would reduce the contact area with NCS (relative to the mass of the crystal). This reduction in relative contact area would reduce the mechanical strength of the bond between the NCS and the $\mathrm{Cu}_{2}\left(\mathrm{NO}_{3}\right)(\mathrm{OH})_{3}$ crystal, facilitating their separation during sample recovery or preparation of the sample for electron microscopy.

The distinct separation of the copper crystalline phase and NCS is observed even when the total copper uptake is minimal. Figure 3.17 shows the EDS overlay for the sorption of copper nitrate by a $\mathrm{pH}$ adjusted NCS sample. The NCS and the $\mathrm{Cu}_{2}\left(\mathrm{NO}_{3}\right)(\mathrm{OH})_{3}$ crystals are distinct from the NCS, and are of a similar size to those observed as a result of sorption with 2-ethoxyethanol washed NCS. While it is still likely that NCS provides the initial nucleation site for crystal formation, $\mathrm{Cu}_{2}\left(\mathrm{NO}_{3}\right)(\mathrm{OH})_{3}$ acts as a preferential substrate for its own growth.

The EDS overlay of NCS following sorption of $\mathrm{Cu}^{2+}$ from an $80 \mathrm{mmol} \mathrm{dm}{ }^{-3} \mathrm{CuCl}_{2}$ solution is shown in Figure 3.18. The EDS was recorded subsequent to the measurement of the 10000 times magnification electron micrograph presented in Figure 3.14. In addition to silicon (red) and copper (green), the chlorine elemental distribution was also recorded (blue). These colour assignments mean that the overlay image appears cyan where copper and chlorine are in close proximity; yellow where silicon and copper are in close proximity; magenta where silicon and chlorine are in close proximity; and white where all three elements are in close proximity. 

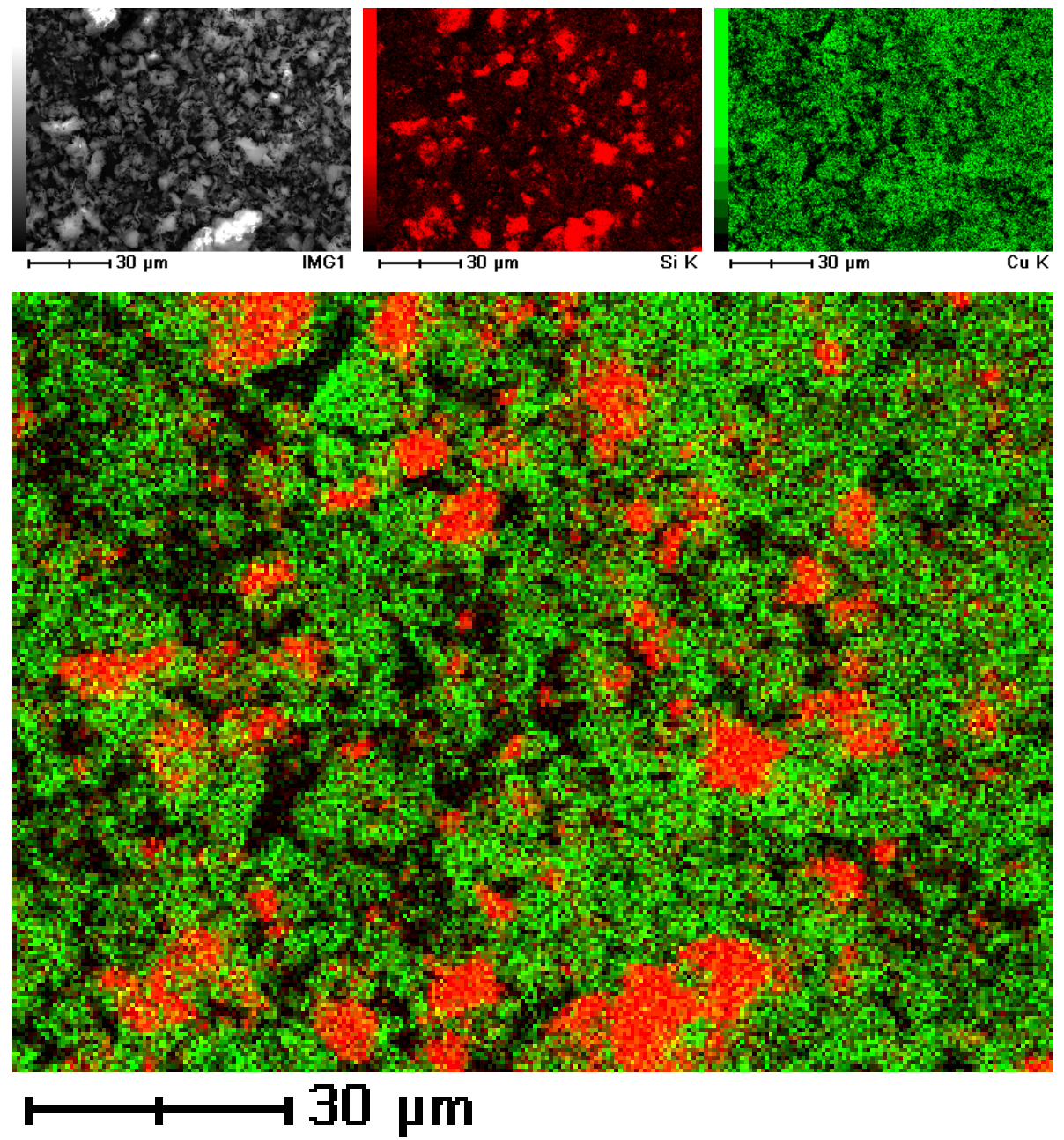

Figure 3.16 EDS overlay of NCS following sorption of $\mathrm{Cu}^{2+}$ from an $80 \mathrm{mmol} \mathrm{dm}^{-3}$ $\mathrm{Cu}\left(\mathrm{NO}_{3}\right)_{2}$ solution: $\mathrm{Si}$ (red); $\mathrm{Cu}$ (green). 

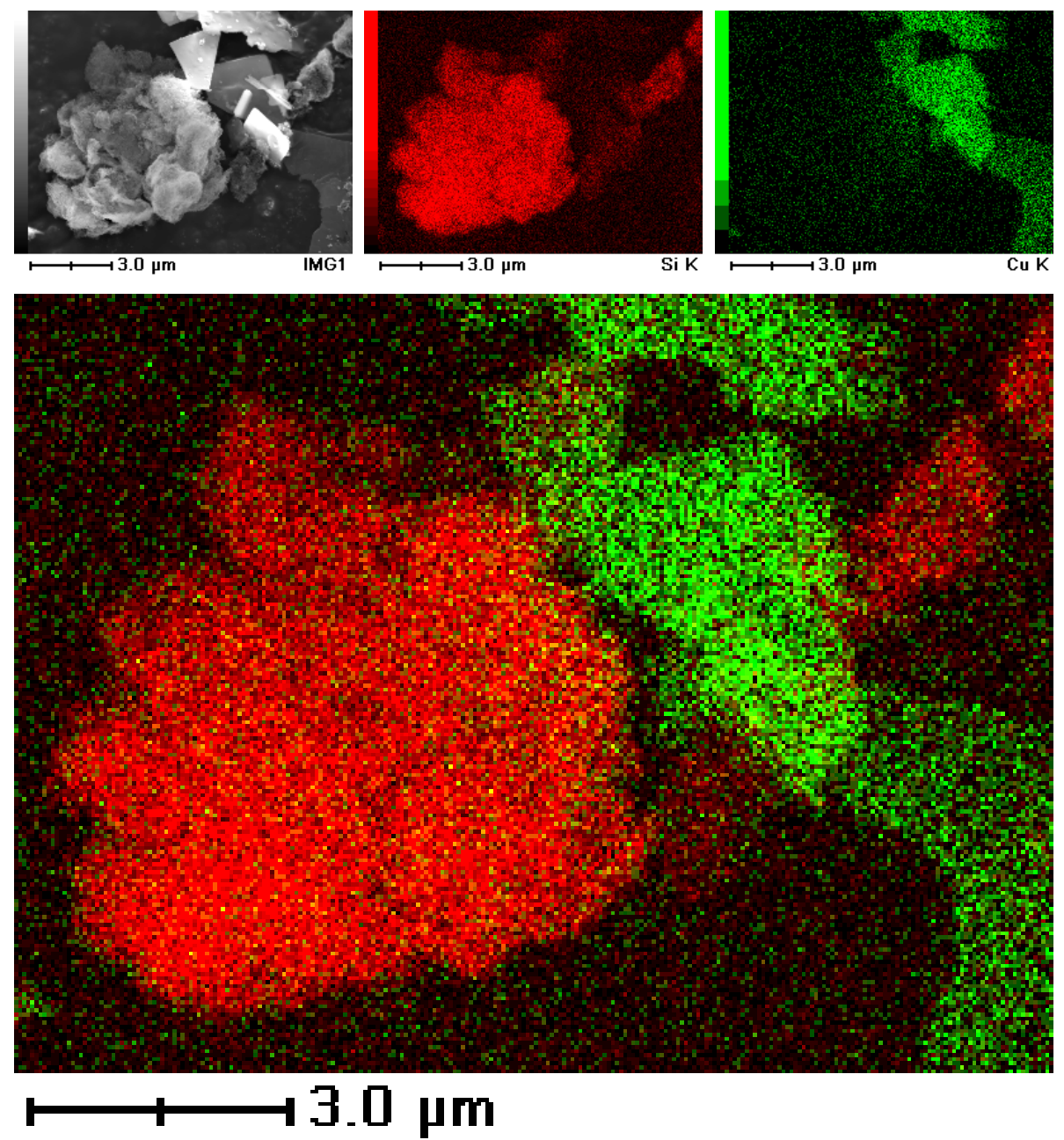

Figure 3.17 EDS overlay of $\mathrm{pH}$ adjusted NCS following sorption of $\mathrm{Cu}^{2+}$ from an $80 \mathrm{mmol} \mathrm{dm}{ }^{-3} \mathrm{Cu}\left(\mathrm{NO}_{3}\right)_{2}$ solution: $\mathrm{Si}$ (red); $\mathrm{Cu}$ (green). 
It can be seen in the figure that the majority of the NCS particle is white, meaning that all three elements are in close proximity. ${ }^{\dagger}$ As the EDS technique records X-rays, generated when an electron beam interacts with atoms in the sample, the vertical resolution of the EDS technique is limited by the penetration depth of the electron beam. This penetration distance can be several hundreds of nanometres. Therefore, the white colour observed is consistent with crystals of $\mathrm{Cu}_{2} \mathrm{Cl}(\mathrm{OH})_{3}$ located on the surface of the NCS particle. This may also be seen near the edges of the particle, where silicon free regions are evidenced by the presence of cyan regions in the image. There are also red areas in the image, indicating regions of the sample where no copper sorption is observed. It is possible that these regions were obscured during the sorption experiment (possibly towards the interior of an NCS particle) and were subsequently exposed during the experimental steps involving recovery of the spent sorbent, its drying and preparation for electron microscopy.

This is in contrast to the growth of the $\mathrm{Cu}_{2}\left(\mathrm{NO}_{3}\right)(\mathrm{OH})_{3}$ crystals observed in the sorption experiments from copper nitrate. In those experiments, the copper phase and NCS phase were usually very distinct. In the case of copper chloride however, the $\mathrm{Cu}_{2} \mathrm{Cl}(\mathrm{OH})_{3}$ phases are distributed across the NCS surface sample.

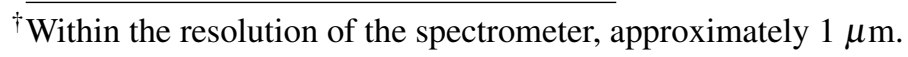



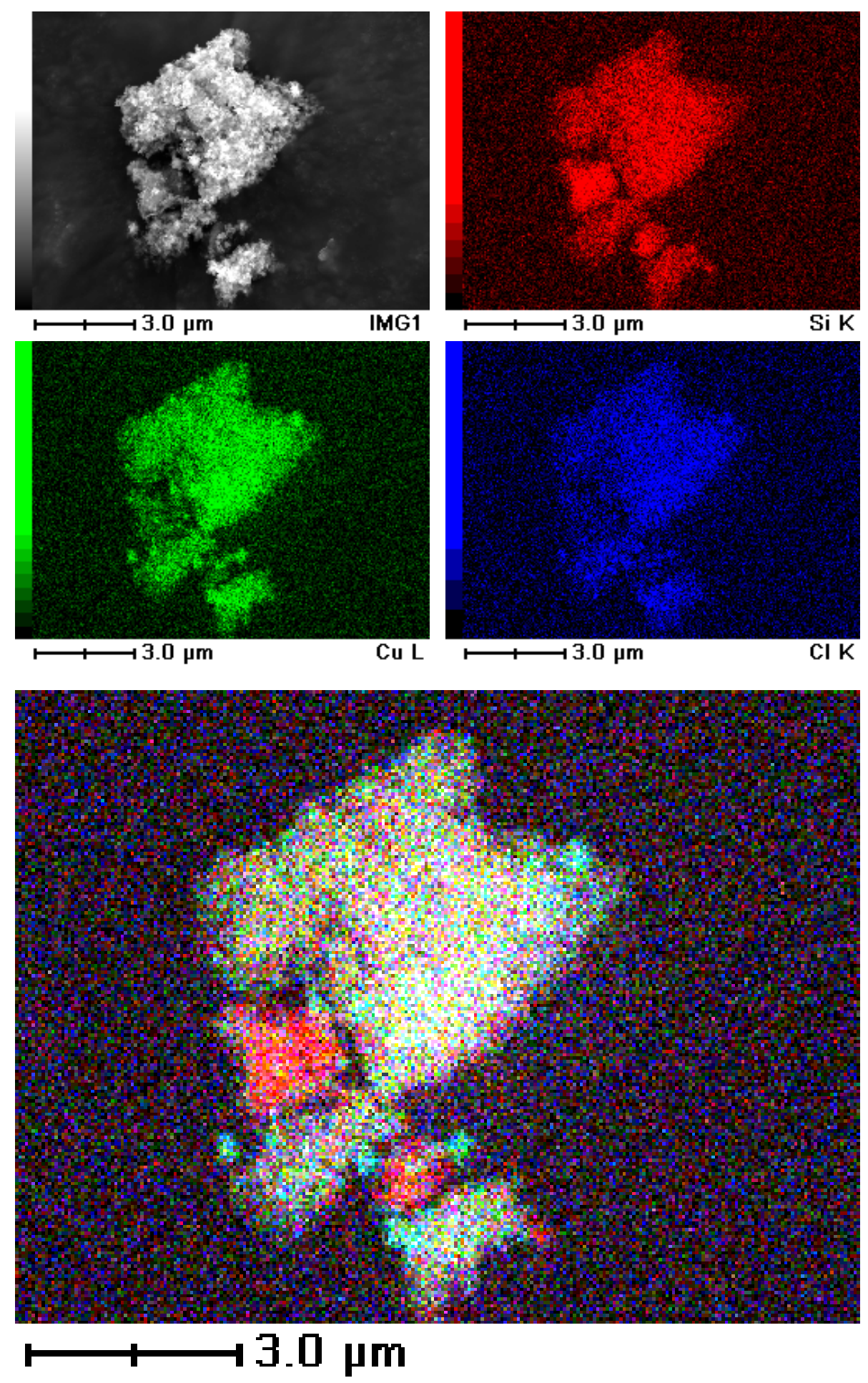

Figure 3.18 EDS overlay of NCS following sorption of $\mathrm{Cu}^{2+}$ from an $80 \mathrm{mmol} \mathrm{dm}^{-3}$ solution $\mathrm{CuCl}_{2}$ : $\mathrm{Si}$ (red); $\mathrm{Cu}$ (green); $\mathrm{Cl}$ (blue). 


\subsubsection{Mechanism of Sorption}

From XRD analysis of the sorbed material, it was noted that copper formed crystalline phases with the molecular formula $\mathrm{Cu}_{2} \mathrm{X}(\mathrm{OH})_{3}$, where $\mathrm{X}$ is either $\mathrm{Cl}^{-}$or $\mathrm{NO}_{3}^{-}$, depending on the counterion present in the initial copper solution. The uptake of copper was accompanied by an associated release of calcium from NCS. Experiments conducted with no copper present revealed that the release of calcium resulted in an increase of the solution $\mathrm{pH}$. In these blank leaching experiments, the increase in hydroxide ion concentration was less than one tenth that of the increase in calcium concentration. Final solution concentrations were $\left[\mathrm{OH}^{-}\right]=0.1 \mathrm{mmol} \mathrm{dm}^{-3},\left[\mathrm{Ca}^{2+}\right]=1.33 \mathrm{mmol} \mathrm{dm}^{-3}$.

As calcium is released from NCS due to its reaction with water charge balance must be maintained, namely: ${ }^{\dagger, \ddagger}$

$$
(\mathrm{CaO})_{0.8} \mathrm{SiO}_{2}+2 x \mathrm{H}_{2} \mathrm{O} \rightleftharpoons(\mathrm{CaO})_{(0.8-x)}(\mathrm{OH})_{2 x} \mathrm{SiO}_{(2-x)}+x \mathrm{Ca}^{2+}+2 x \mathrm{OH}^{-}
$$

In effect, ion exchange of $\mathrm{Ca}^{2+}$ for $\mathrm{H}^{+}$occurs. From 3.18, twice as much hydroxide as calcium should be released. As this was found not to be the case, direct reaction of NCS and $\mathrm{H}^{+}$is unlikely:

$$
\begin{aligned}
& (\mathrm{CaO})_{0.8} \mathrm{SiO}_{2}+2 x \mathrm{H}^{+} \rightleftharpoons(\mathrm{CaO})_{(0.8-x)}(\mathrm{OH})_{2 x} \mathrm{SiO}_{(2-x)}+x \mathrm{Ca}^{2+} \\
& \mathrm{H}_{2} \mathrm{O} \rightleftharpoons \mathrm{H}^{+}+\mathrm{OH}^{-}
\end{aligned}
$$

The discrepancy between the $\mathrm{Ca}^{2+}$ and $\mathrm{OH}^{-}$concentrations may be accounted for if the release of calcium from the NCS occurs in two separate steps, with the NCS material

\footnotetext{
${ }^{\dagger}$ For reasons of clarity, the water coordinated to calcium in NCS (see Figure 1.2) is not shown. Thus, NCS is represented below as $(\mathrm{CaO})_{0.8} \mathrm{SiO}_{2}$, rather than $\mathrm{Ca}_{0.8} \mathrm{SiO}_{2.8} \cdot 2 \mathrm{H}_{2} \mathrm{O}$.

${ }^{\ddagger}$ Calcium and hydrogen are bound within the NCS as $\mathrm{Si}-\mathrm{O}-\mathrm{Ca}--$ and $\mathrm{Si}-\mathrm{O}-\mathrm{H}$.
} 
attaining a negative surface charge:

$$
\begin{aligned}
& (\mathrm{CaO})_{0.8} \mathrm{SiO}_{2} \rightleftharpoons x \mathrm{Ca}^{2+}+(\mathrm{CaO})_{(0.8-x)} \mathrm{SiO}_{(2+x)}^{2 x-} \\
& (\mathrm{CaO})_{(0.8-x)} \mathrm{SiO}_{(2+x)}^{2 x-}+2 x \mathrm{H}_{2} \mathrm{O} \rightleftharpoons(\mathrm{CaO})_{(0.8-x)}(\mathrm{OH})_{2 x} \mathrm{SiO}_{(2-x)}+2 x \mathrm{OH}^{-}
\end{aligned}
$$

The hydroxide may also subsequently react with the silicate backbone, eventually releasing monomeric silica into solution. For most silicate materials, this occurs on timescales of days to weeks: ${ }^{31}$

$$
(\mathrm{CaO})_{(0.8-x)} \mathrm{SiO}_{2}(\mathrm{OH})_{x}+(4-x) \mathrm{OH}^{-} \rightleftharpoons(\mathrm{CaO})_{(0.8-x)} \mathrm{Si}_{(1-x)} \mathrm{O}_{2}^{(4-x)-}+\mathrm{Si}(\mathrm{OH})_{4}(\mathrm{aq})
$$

The release of $\mathrm{SiO}_{x}(\mathrm{OH})_{(4-x)}^{x_{-}}$into solution most likely occurs over a long time scale, as is the case with other silicates. ${ }^{31}$ The $\mathrm{pH}$ of solution (and hence $\left[\mathrm{OH}^{-}\right]$) reaches equilibrium after about 30-60 minutes, whereas the calcium concentration requires several hours to equilibrate. As such, it is unlikely that the $\mathrm{SiO}_{x}(\mathrm{OH})_{(4-x)}^{x_{-}}$release contributes significantly to the discrepancy between the calcium and hydroxide concentrations.

However, if copper is present in solution, the copper will compete for the released hydroxide, forming copper hydroxide phases:

$$
x \mathrm{Cu}^{2+}+y \mathrm{OH}^{-}+(2 x-y) \mathrm{NO}_{3}^{-} \longrightarrow \mathrm{Cu}_{x}(\mathrm{OH})_{y}\left(\mathrm{NO}_{3}\right)_{(2 x-y)}
$$

Experiments conducted at an initial copper concentration of $1.6 \mathrm{mmol} \mathrm{dm}^{-3}$ presented as amorphous in XRD studies. These materials removed all copper from solution, meaning the XRD studies were conducted on samples containing $1.6 \mathrm{mmol} \mathrm{Cu}^{2+} \mathrm{g}^{-1}$ initial NCS ( 9 wt $\% \mathrm{Cu}$ in the final material). As no crystalline materials could be detected by XRD in a sample containing $9 \mathrm{wt} \%$ copper, it is likely that the initial copper precipitates formed are amorphous. The crystalline phases will form only in the presence of an excess of copper.

In cases of maximum copper uptake, the copper will form a crystalline phase with a 
$\mathrm{Cu}^{2+}: \mathrm{OH}^{-}$ratio of $2: 3$, such as $\mathrm{Cu}_{2}\left(\mathrm{NO}_{3}\right)(\mathrm{OH})_{3}$. If it is assumed that the only source of hydroxide is provided as a result of leaching $\mathrm{Ca}^{2+}$ into solution at a $\mathrm{Ca}^{2+}: \mathrm{OH}^{-}$ratio of $1: 2$, then the ratio of copper sorbed to calcium released should be $\mathrm{Cu}^{2+}: \mathrm{Ca}^{2+}=4: 3$ in an ideal case of maximum sorption. The NCS material contains approximately $28 \mathrm{wt} \%$ calcium, or $7.1 \mathrm{mmol} \mathrm{g}^{-1}$. If all this calcium were to be released, a copper sorption of $9.4 \mathrm{mmol} \mathrm{g}^{-1}$ should result. The experimental data differ slightly from this ideal scenario.

The maximum recorded copper sorption capacity in a continuously stirred experiment was $10 \pm 1 \mathrm{mmol} \mathrm{Cu}^{2+} \mathrm{g}^{-1}$, associated with a calcium release of $6.5 \mathrm{mmol} \mathrm{Ca}^{2+} \mathrm{g}^{-1}$. At the $4: 3$ exchange ratio, this calcium release should be associated with $8.6 \mathrm{mmol} \mathrm{g}^{-1}$ copper uptake. This is consistent with the reported copper uptake.

The presence of an X-ray amorphous product (at low initial copper concentrations) and a crystalline material (at initial copper concentrations) is indictive of two different sorption mechanisms. At low copper concentrations, the copper is distributed evenly throughout the spent sorbent, whereas with an excess of copper, distinct crystalline copper phases form. Only in the prior case could the mechanism be considered actual sorption. The latter case is more akin to a precipitation reaction, as evidenced by the leaching of all calcium present in the NCS.

The reaction kinetics applied to the release of hydroxide indicated a different mechanism in the presence or absence of copper. In the absence of copper, the release of hydroxide exhibited adequate conformity to both a first- or second- order model. It is likely that the observed kinetics with respect to hydroxide in the absence of copper may be a filmdiffusion mechanism, equivalent to first-order kinetics.

In cases where copper is present, the release of hydroxide exhibits zeroth order kinetics. This steady release of hydroxide suggests that some other component of the system is rate-limiting. If release of $\mathrm{Ca}^{2+}$ from the NCS were the rate-limiting factor, it would be expected that there should always be an excess of $\mathrm{Cu}^{2+}$ near the NCS surface. Thus, as the calcium release occurs, the associated hydroxide would react with the excess of copper. This would lead to no observed change in the bulk hydroxide concentration, which is not the case. 
If the system was instead limited by the diffusion of copper towards the NCS, it would be expected that there would always be an excess of $\mathrm{Ca}^{2+}$ and $\mathrm{OH}^{-}$in solution near the NCS. This excess surface hydroxide concentration would lead to the diffusion of hydroxide into the bulk solution. If the excess $\mathrm{OH}^{-}$concentration were significantly greater than the bulk solution concentration (as might be expected when the bulk solution is acidic or near-neutral), then diffusion of $\mathrm{OH}^{-}$from the NCS surface would exhibit zeroth order kinetics, consistent with Fick's first law (Equation (1.22)). When iodine was added to the sorption system, the rate of $\mathrm{Ca}^{2+}$ release increased and the rate of $\mathrm{OH}^{-}$release decreased. By comparison, no significant change was noted in the rate of $\mathrm{Cu}^{2+}$ uptake. This result is consistent with the system being rate-limited by the diffusion of copper.

\subsection{Conclusions}

It was found that NCS rapidly sorbed both chloride and nitrate salts of copper. Under dynamic conditions, the NCS material sorbed copper with a maximum sorption capacity of $9 \pm 1 \mathrm{mmol} \mathrm{Cu}^{2+} \mathrm{g}^{-1} \mathrm{NCS}$ for $\mathrm{CuCl}_{2}$, and $10 \pm 1 \mathrm{mmol} \mathrm{Cu}^{2+} \mathrm{g}^{-1} \mathrm{NCS}$ for $\mathrm{Cu}\left(\mathrm{NO}_{3}\right)_{2}$. When sorption was conducted in the presence of excess copper, the crystalline phases atacamite $\left(\mathrm{Cu}_{2} \mathrm{Cl}(\mathrm{OH})_{3}\right)$ and rouaite $\left(\mathrm{Cu}_{2}\left(\mathrm{NO}_{3}\right)(\mathrm{OH})_{3}\right)$ were observed, depending on the initial copper salt. In cases where sufficient NCS was applied to completely sorb the copper, no crystalline copper phases were observed by X-ray diffraction, even though such materials contained $7.8 \pm 0.2 \mathrm{mmol} \mathrm{Cu}{ }^{2+} \mathrm{g}^{-1} \mathrm{NCS}$. Electron microscopy studies demonstrated the initial formation of crystalline copper phases. In the case of copper nitrate, it was found that, when the crystalline $\mathrm{Cu}_{2}\left(\mathrm{NO}_{3}\right)(\mathrm{OH})_{3}$ phase formed, it did so as large micron-sized crystals, comparable to the size of the NCS particles themselves. As the crystalline copper phase formed, the elemental distribution of the materials changed: In an excess of copper nitrate, the copper was only present as a crystalline material and was distinct from the NCS. A deficit of copper led to a homogeneous elemental distribution of copper throughout the NCS sample. By comparison, sorption of copper chloride showed that $\mathrm{Cu}_{2} \mathrm{Cl}(\mathrm{OH})_{3}$ only formed crystallites in the size range of a few tens of nanometres, with a relatively even distribution across the NCS surface. 
With an initial copper concentration of $1.6 \mathrm{mmol} \mathrm{dm}^{-3}, 1 \mathrm{~g} \mathrm{dm}^{-3} \mathrm{NCS}$ can remove all detectable copper from solution in approximately 30-60 minutes (a removal efficiency greater than $99.5 \%$ ). At a higher initial copper concentration of $7.8 \mathrm{mmol} \mathrm{dm}^{-3}$, NCS applied at the same loading only results in the removal of $74 \%$ of the initial copper.

It was demonstrated that the presence of calcium was required for copper sorption. In cases where most of the calcium was leached from NCS with $\mathrm{HCl}$ prior to sorption studies, the maximum sorption capacity achieved was $2 \pm 1 \mathrm{mmol} \mathrm{Cu}^{2+} \mathrm{g}^{-1} \mathrm{NCS}$. In the presence of excess copper nitrate, the formation of crystalline phases was still observed. Experiments were conducted in the presence of iodine as a means of increasing the activity of NCS through the formation of $\mathrm{Ca}-\mathrm{I}$ complexes. It was found that the presence of $\mathrm{I}_{3}^{-}$within the system promoted the rate of calcium leaching from the NCS material. Under the conditions tested, the rate at which copper was sorbed from solution was not altered to the same degree. This suggested that under these conditions the copper removal was controlled by the rate of diffusion of $\mathrm{Cu}^{2+}$ towards the NCS surface.

Kinetic modelling of the copper removal or calcium release could not adequately distinguish between first- or second- order kinetics. While calculated $R^{2}$ values were consistently greater for the second-order models, the difference was usually less than 0.05. All calculated $R^{2}$ values for calcium release (both first- and second-order) were greater than 0.9. For the copper uptake, the $R^{2}$ values were in the range of $0.841-0.982$. The calculated values for copper were less than those for calcium in every case. In the first-order kinetic models, it was found that the rate constant for calcium release increased with an increase in the initial copper concentration; the first-order rate constant for copper decreased with an increase in the initial copper concentration. This indicated that the mechanism did not follow simple first-order kinetics, as in a simple first-order model the rate constant is independent of the concentration of the species concerned. Zeroth-order kinetics were observed for the release of $\mathrm{OH}^{-}$into solution during copper sorption by NCS. This is also consistent with the system being rate limited by the diffusion of copper from the bulk of the solution, to the NCS surface. 


\section{Magnetic Calcium Silicate Composites}

\subsection{Introduction}

\subsubsection{Magnetism}

A magnetic field is generated as the result of a moving charge, existing in a plane perpendicular to the plane of electric charge. A current (i), moving in a circular path of radius $r$ will generate a magnetic field $(H)$, at its centre. Associated with this magnetic field are a magnetic moment $(\mu)$, magnetisation $(M)$, magnetic susceptibility $(\chi)$, magnetic moment per unit mass $(\sigma)$ and flux density $(B)$. These terms are given in Table 4.1.

Table 4.1 Definition of some quantities associated with magnetism (after Dunlop and Özdemir $\left.{ }^{105}\right)$.

\begin{tabular}{|c|c|c|}
\hline \multirow{2}{*}{$\begin{array}{l}\text { Quantity } \\
\text { Magnetic field, } H / \mathrm{A} \mathrm{m}^{-1}\end{array}$} & \multicolumn{2}{|c|}{ Definition } \\
\hline & $H=\frac{i}{2 r}$ & $(4.1)$ \\
\hline $\begin{array}{l}\text { Magnetic moment (current bisect- } \\
\text { ing unit area), } \mu / \mathrm{A} \mathrm{m}^{2}\end{array}$ & $\mu=i A$ & $(4.2)$ \\
\hline $\begin{array}{l}\text { Magnetisation (magnetic moment } \\
\text { per unit volume), } M / \mathrm{A} \mathrm{m}^{-1}\end{array}$ & $M=\frac{\mu}{V}$ & $(4.3)$ \\
\hline Magnetic susceptibility $\chi$ & $\chi=\frac{M}{H}$ & $(4.4)$ \\
\hline $\begin{array}{l}\text { Magnetic moment per unit mass, } \sigma / \\
\mathrm{A} \mathrm{m}^{2} \mathrm{~kg}^{-1}\end{array}$ & $\sigma=\frac{M}{\rho}=\frac{\mu}{m}$ & $(4.5)$ \\
\hline Flux density, $B / \mathrm{T} \equiv \mathrm{N} \mathrm{A}^{-1} \mathrm{~m}^{-1}$ & $B=\mu_{0}(H+M)$ & $(4.6)$ \\
\hline $\begin{array}{l}\text { Permeability of free space, }{ }^{a} \mu_{0} / \\
\mathrm{H} \mathrm{m}^{-1} \equiv \mathrm{N} \mathrm{A}^{-2}\end{array}$ & $\mu_{0}=4 \pi \times 10^{-7}$ & \\
\hline
\end{tabular}

\footnotetext{
${ }^{a}$ Under the centimetre-gram-second (CGS) system of units, still commonly used for electromagnetic quantities, $\mu_{0}$ is defined as unity. Hence $\chi_{\mathrm{SI}}=4 \pi \chi_{\mathrm{CGS}}$ although the quantity is dimensionless under both the SI and CGS systems.
}

The quantum view of magnetism is similar in that a moving charged particle (such 
as an electron) will generate a current and, hence, have a magnetic moment. As the angular velocity and angular momentum of the particles are quantised, with the minimum quantum being the reduced form of Planck's constant $\left(\hbar=\frac{h}{2 \pi}\right)$, the magnetic moment is also quantised. The minimum magnetic moment possessed by an electron is the Bohr Magneton:

$$
\mu_{B}=\frac{e \hbar}{2 m_{e}}=9.274 \times 10^{-24} \mathrm{~A} \mathrm{~m}^{2}
$$

\subsubsection{Ferrimagnetism and Antiferrimagnetism}

Ferrimagnetism, first proposed by Néel, ${ }^{106}$ occurs when a crystalline material contains two sublattices. Within each sublattice, the magnetic moments of paramagnetic entities are aligned parallel, but the magnetic moments of the two sublattices are aligned antiparallel. If the sublattices have magnetic moments of unequal magnitude, the bulk material will possess a non-zero magnetic moment. Examples of ferrimagnetic materials are magnetite, $\mathrm{Fe}_{3} \mathrm{O}_{4}$, and maghemite, $\gamma-\mathrm{Fe}_{2} \mathrm{O}_{3}$. If the two sublattices have an equal magnetic moment, the material will exhibit a net magnetic moment of zero. This class of materials are known as antiferrimagnets. Hematite, $\alpha-\mathrm{Fe}_{2} \mathrm{O}_{3}$, is an example of such a material. By comparison, ferromagnetic materials, such as iron, consist of only one magnetic lattice within the crystal structure. The $3 \mathrm{~d}$ orbitals of atoms in iron metal do not overlap. Therefore, the unpaired $3 \mathrm{~d}$ electrons of adjacent $\mathrm{Fe}$ atoms may be aligned parallel (the lower energy state) without violating Pauli's exclusion principle.

Magnetite exists in the inverse spinel structure, ${ }^{107}$ which may be represented by the formula $\left[\mathrm{Fe}^{\mathrm{III}}\right]_{\mathrm{A}}\left[\mathrm{Fe}^{\mathrm{II}} \mathrm{Fe}^{\mathrm{III}}\right]_{\mathrm{B}} \mathrm{O}_{4}$, where $\mathrm{A}$ and $\mathrm{B}$ represent tetrahedral and octahedral sites respectively, within the crystal structure. The two sublattices (A and B) arise due to overlap of the iron $3 \mathrm{~d}$ orbitals with an intermediate oxide $2 \mathrm{p}$ orbital. To avoid violation of Pauli's exclusion principle, the electrons in each $3 \mathrm{~d}$ orbital are aligned antiparallel in respect to their 'partner' electron in the oxygen $2 \mathrm{p}$ orbital. This has the net result of aligning the $3 \mathrm{~d}$ electrons in the octahedral cation sites antiparallel to those in the tetrahedral cation sites. Due to the antiparallel arrangement of $3 \mathrm{~d}$ electron spin between the $\mathrm{A}$ and $\mathrm{B}$ sites, there is zero net contribution to the magnetic moment of magnetite 
from $\mathrm{Fe}^{3+}$ as the contribution of $\mathrm{Fe}_{\mathrm{A}}^{3+}$ cancels that of $\mathrm{Fe}_{\mathrm{B}}^{3+}$. The net magnetic moment of magnetite arises as unpaired $3 \mathrm{~d}$ electrons of the octahedral $\mathrm{Fe}^{2+}$ ions are aligned parallel to each other. This is shown in Figure 4.1.

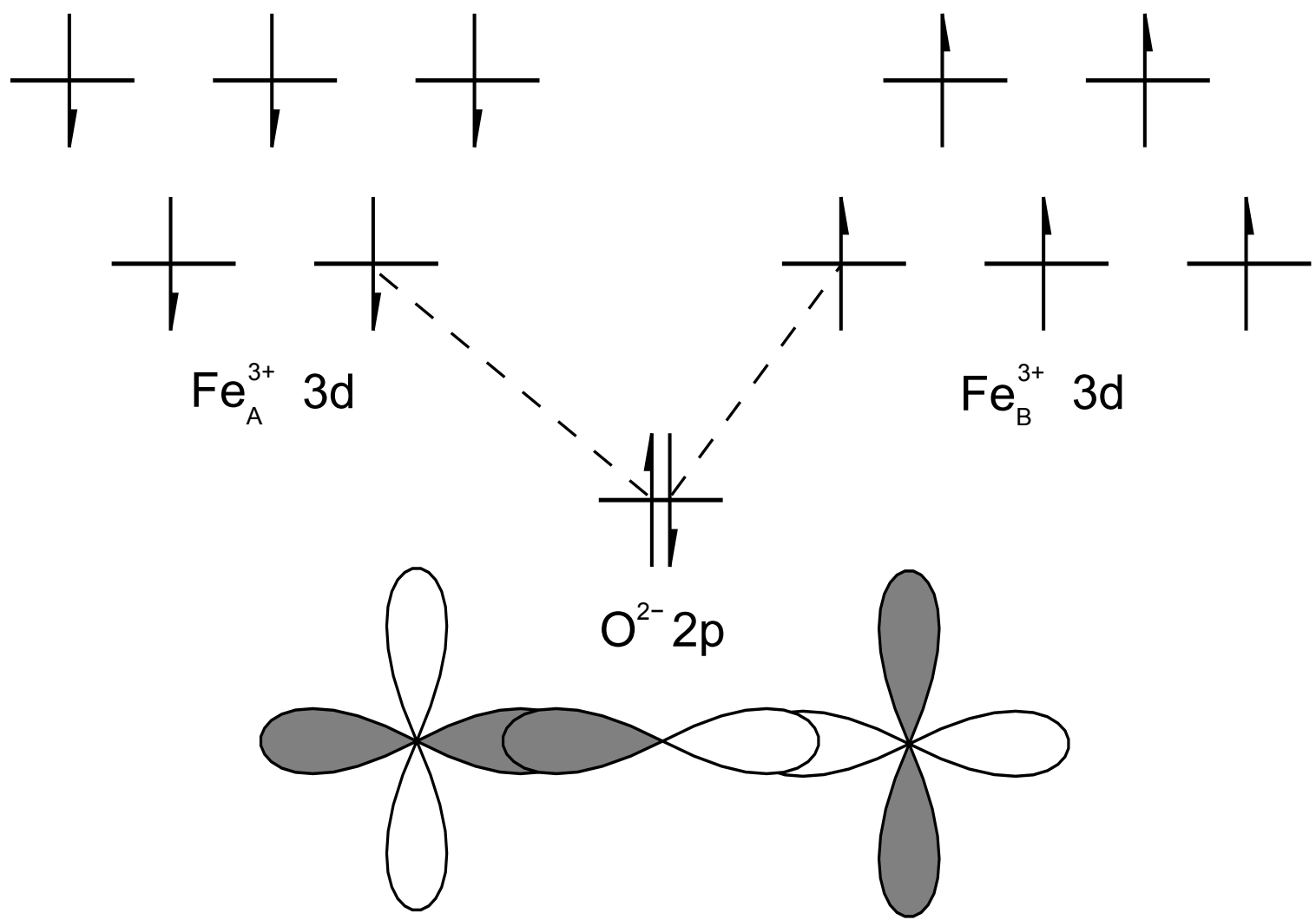

Figure 4.1 Formation of magnetic sub-lattices within the magnetite structure due to antiparallel alignment of electron spins in the tetrahedral (A) and octahedral (B) cation sites. The net magnetic moment of magnetite arises due to the presence of $\mathrm{Fe}^{2+}$ in the octahedral (B) cation site (not shown).

\subsubsection{Macroscopic Properties}

The presence of long range alignment of magnetic moments within a ferro- or ferrimagnetic material can lead to significant charge separation at the surface of the material. This energetically unfavourable charge dipole may be reduced through the formation of magnetic domains, a region of a material having a uniform (non zero) magnetic moment. Magnetic domains possess a finite maximum size, dependant on the magnetisation of the material. Materials with a higher magnetic moment will form smaller domains as the magnitude of charge separation is proportional to the magnetic moment of the material. For magnetite, the maximum domain size is approximately $80 \mathrm{~nm}$. The formation of domains within a crystal, reducing local surface charge, is shown in Figure 4.2 
Magnetism is dependant on the parallel alignment of electron spin states, which in turn are dependant on the orientation of molecular orbitals within the material. As a result, magnetisation vectors are usually preferentially aligned with certain crystalline planes. This can lead to anisotropy within the material due to the energy barrier associated with aligning the magnetisation vector along an unfavourable crystalline axis. Crystallite shape also contributes to anisotropy. If the magnetic moment is aligned along the major axis of an ellipsoidal grain, the charge dipole is reduced, compared with alignment along the minor axis. The magnetisation vectors preferentially align along the major axis of an ellipsoidal crystallite.

The presence of magnetic domains within a sample leads to the hysteresis characteristics shown in Figure 4.3. The curve exhibiting hysteresis (red) was measured on a bulk nickel sample. Saturation magnetisation $\left(\sigma_{s}\right.$ or $\left.M_{S}\right)$, along with magnetic remanence $\left(\sigma_{r}\right.$ or $\left.M_{r}\right)$ and coercivity $\left(H_{c}\right)$ are shown. Remnance is the magnetic moment (or magnetisation) present at zero field, while coercivity is the reverse field that must be applied to reduce the magnetic moment to zero. Saturation magnetisation is reached when the magnetic field is sufficiently strong to align all domain moments parallel. Even in the absence of a magnetic field, individual domains are at saturation magnetisation. However, their lack of alignment reduces the observed moment. This is in contrast to the magnetisation
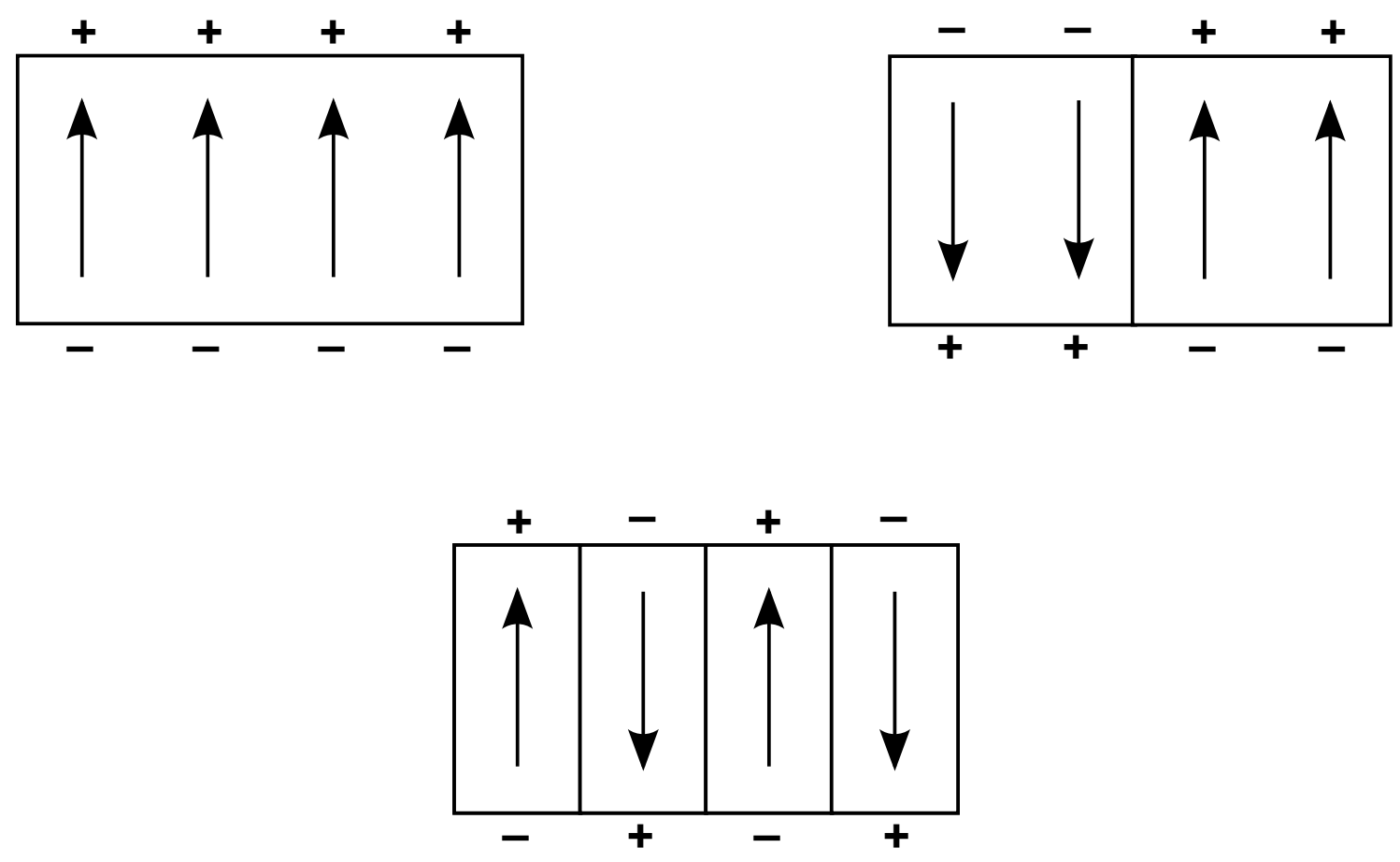

Figure 4.2 Formation of domains within a magnetic crystal. 
curve of a superparamagnetic material (green), discussed below, exhibiting no coercivity or remanence.
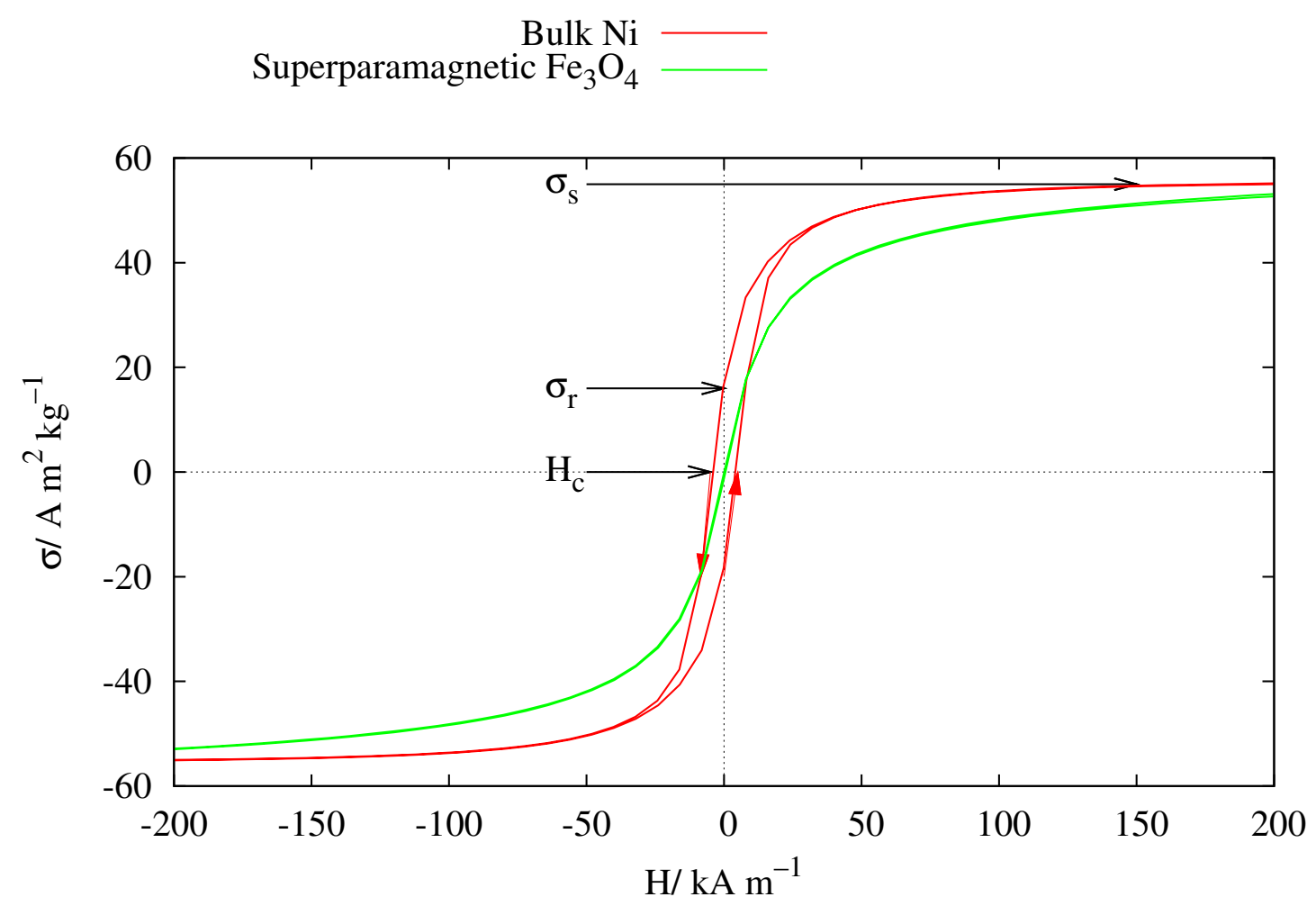

Figure 4.3 Magnetisation curves for a bulk nickel sample (red) and superparamagnetic $\mathrm{Fe}_{3} \mathrm{O}_{4}$ (green). Hysteresis is observed in the nickel curve. Saturation $\left(\sigma_{s}\right)$ and remnant $\left(\sigma_{r}\right)$ magnetic moments, and coercivity $\left(H_{c}\right)$ are indicated for the nickel sample.

\subsubsection{Superparamagnetism}

In the absence of an external magnetic field, the net magnetic moment of an ensemble of ferri- or ferro-magnetic particles will eventually relax to zero. This occurs due to a realignment of the magnetic moment within individual crystallites. Magnetic anisotropy associated with these crystals can impose a significant energy barrier towards this realignment. The relaxation time, $\tau$, for such particles is given in Equation (4.8), ${ }^{106}$ and depends on the particle shape, volume and temperature.

$$
\frac{1}{\tau}=f_{0} \mathrm{e}^{\left(\frac{-K_{u} V}{k T}\right)}
$$

For small particles at a high temperature, the relaxation time is fast, for large particles at 
a low temperature, the relaxation time is slow. $K_{u}$ is the anisotropy constant and depends on both particle composition and shape. For spherical magnetite particles, the value of $K_{u}$ is approximately $1.6 \times 10^{3} \mathrm{~J} \mathrm{~m}^{-3} \cdot f_{0}$ is a frequency constant of approximately $1 \times 10^{9} \mathrm{~s}^{-1} .{ }^{105}$ If the net magnetisation of an ensemble of particles will relax to zero within an experimental timescale of seconds to minutes, ${ }^{\dagger}$ the particles are said to be superparamagnetic. For magnetite particles at room temperature, superparamagnetism usually occurs for particles less than $30 \mathrm{~nm}$ in size.

\subsubsection{Magnetic Separation}

The approach taken towards magnetic separation of particles from suspension depends on their magnetic characteristics. For particles having a superparamagnetic character, the separation requires the presence of an external magnetic field, as the particles have no remnant magnetic moment, and hence will not agglomerate on their own. For larger magnetic particles, separation may be achievable without the requirement for an external magnetic field, although such a field is often used to assist the separation.

\subsubsection{High Gradient Magnetic Separation}

In order to successfully separate particles from suspension, sufficient force must be applied to overcome the forces keeping them in suspension. As small particles possess a high kinetic energy due to Brownian motion, the force of gravity alone is insufficient to separate the particles over timescales of a few seconds. To accomplish this task, additional forces must be applied. If the particles being separated are superparamagnetic, high gradient magnetic separation (HGMS) can be used. ${ }^{108}$

The presence of ferromagnetic objects (a steel wire for example) within a magnetic field will cause a magnetic field gradient to be generated (Figure 4.4). With narrow wires, higher magnetic field gradients can be generated. ${ }^{109}$ When a (superpara)magnetic particle enters this field gradient, an attractive force, given by Equation (4.9)) acts on the particle,

\footnotetext{
$\dagger$ While this is an inexact time scale definition, the exponential dependence in (4.8) on volume means that an increase in particle diameter by a few percent can increase the relaxation time by an order of magnitude.
} 
leading to the particle accumulating on the wire surface. ${ }^{110}$ In this equation, $F$ is the force acting on the particle, $V$, the particle volume, and $\mu_{0}, M$, and $H$ have the same definitions as those given in Table 4.1. Higher magnetic field gradients lead to greater forces acting on the particles to be separated, and hence faster separation. A practical implementation of the HGMS system usually consists of many wires, often as a canister of steel wool, through which the particles to be separated are passed. ${ }^{110,111}$

$$
F=\mu_{0} V M \nabla H
$$

Superparamagnetic particles are usually used for HGMS, as an ensemble of these particles has no net magnetic moment in the absence of a magnetic field. Thus, if the magnetic field is removed the particles can be easily recovered from the separator. This cycling of the magnetic field is most easily accomplished via the use of an electromagnet, ${ }^{110,111}$ although Franzreb et al. ${ }^{112}$ have developed a HGMS system that utilises permanent magnets. It is often desirable to incorporate functionality into a magnetic particle in order to achieve sorbate specificity. For example, Meyer et al. ${ }^{113}$ have incorporated copper within magnetic particles to facilitate the separation of the enzyme superoxide dismutase from whey through a HGMS process. In cases where the magnetic particles are modified to aid the recovery of a specific sorbate, the process is known as magnetically assisted chemical separation.
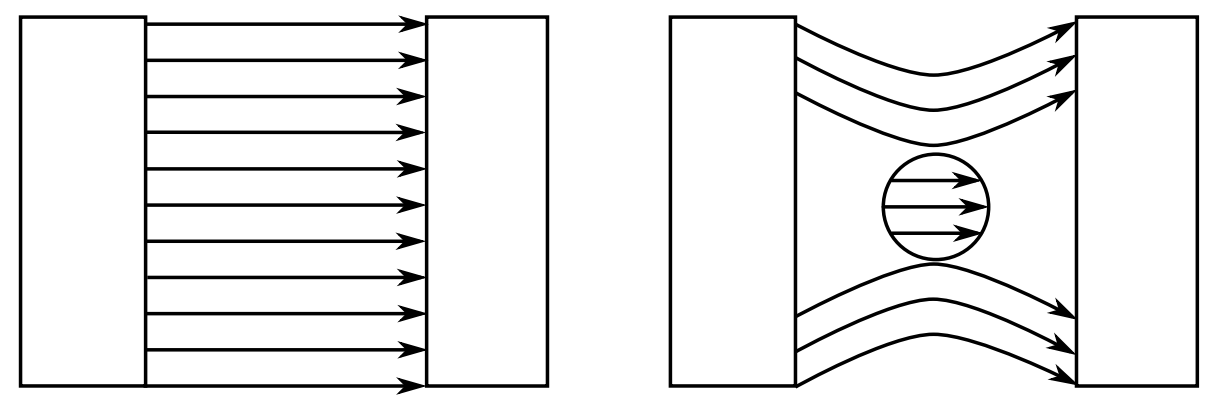

Figure 4.4 Magnetic field gradient generated by the presence of a ferromagnetic wire in an otherwise uniform field. 


\subsubsection{Flocculation}

When the magnetic particles to be separated are large (a few microns in size), separation techniques are often designed so as to make use of the permanent magnetic moment of the particles. ${ }^{114}$ Many techniques rely on the flocculation of the magnetic particles and the subsequent separation of the flocculated product. Priestley, ${ }^{115}$ for example has reported a separation technique for the purification of coloured waters based of the sorption of the colourants onto magnetite, application of a magnetic field to generate a floc, and subsequent sedimentation of the floc by gravity. Pavlova and Dobrevsky ${ }^{116}$ report an adaptation of this method for the separation of humic acids from natural waters.

Franzreb et al. ${ }^{117}$ also report the use of a magnetic flocculation system to remove phosphate from sewage. By adding $\mathrm{Fe}^{3+}$ and magnetite to a sewage sample, the authors were able to form magnetite-iron phosphate flocs, removed with the use of a rotatingdrum type HGMS separator. A similar concept has been demonstrated by Feng et al. ${ }^{89}$ where magnetite and $\mathrm{Fe}^{3+}$ were added to a copper-sorbed zeolite, inducing formation of a magnetic floc.

\subsection{Results}

The characterisation of a series of magnetic NCS composites is presented. The adsorption characteristics of the $\gamma-\mathrm{Fe}_{2} \mathrm{O}_{3}$-NCS with respect to copper are reported and these results compared to those of the unmodified NCS material.

\subsubsection{Superparamagnetic NCS Composites}

\subsubsection{Particle Size Analysis}

Figure 4.5 shows the particle size distribution for a series of $\mathrm{Fe}_{3} \mathrm{O}_{4}$-NCS composites prepared with a nanosized, superparamagnetic $\mathrm{Fe}_{3} \mathrm{O}_{4}$ suspension. Concentrations of the 


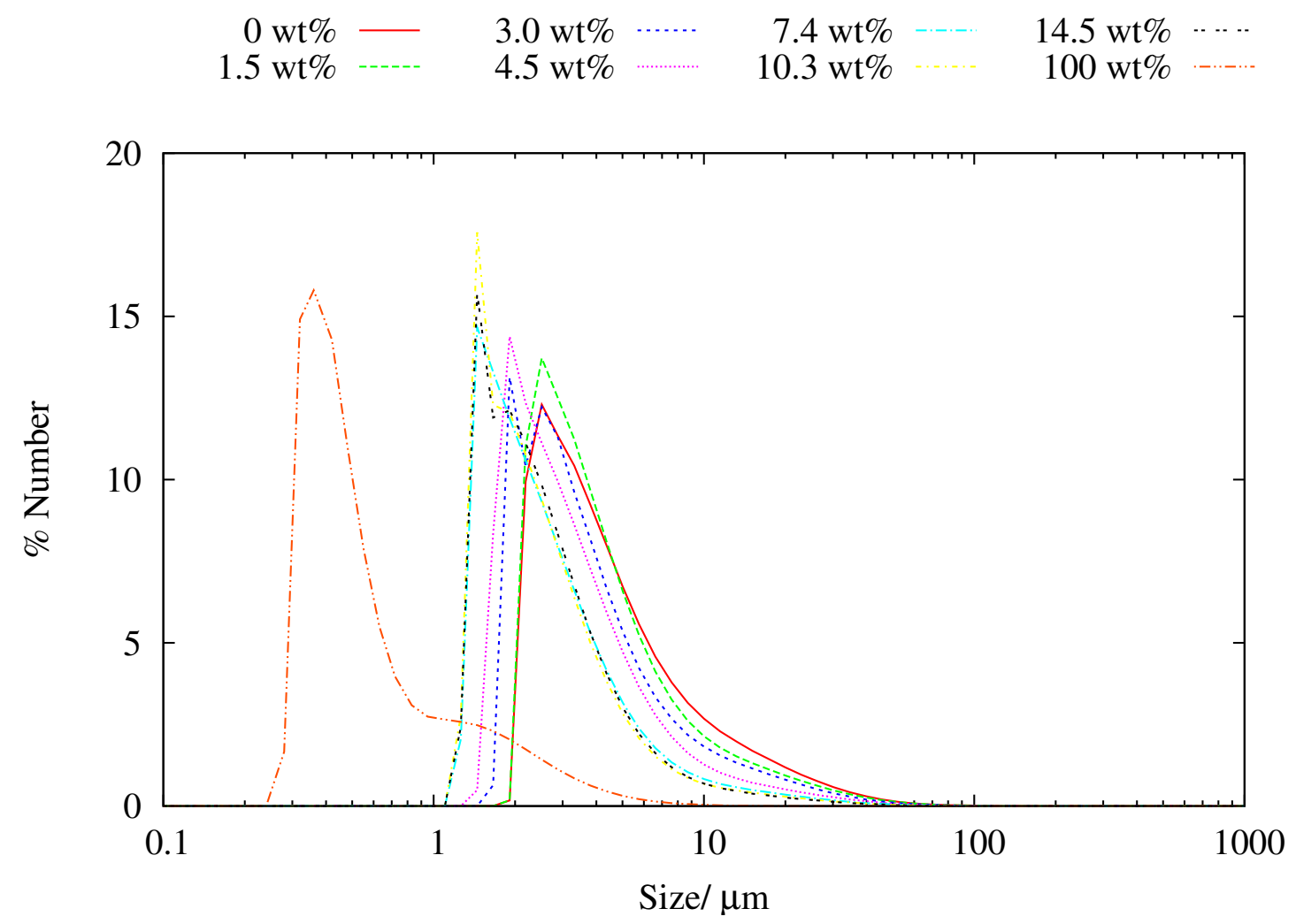

(a)

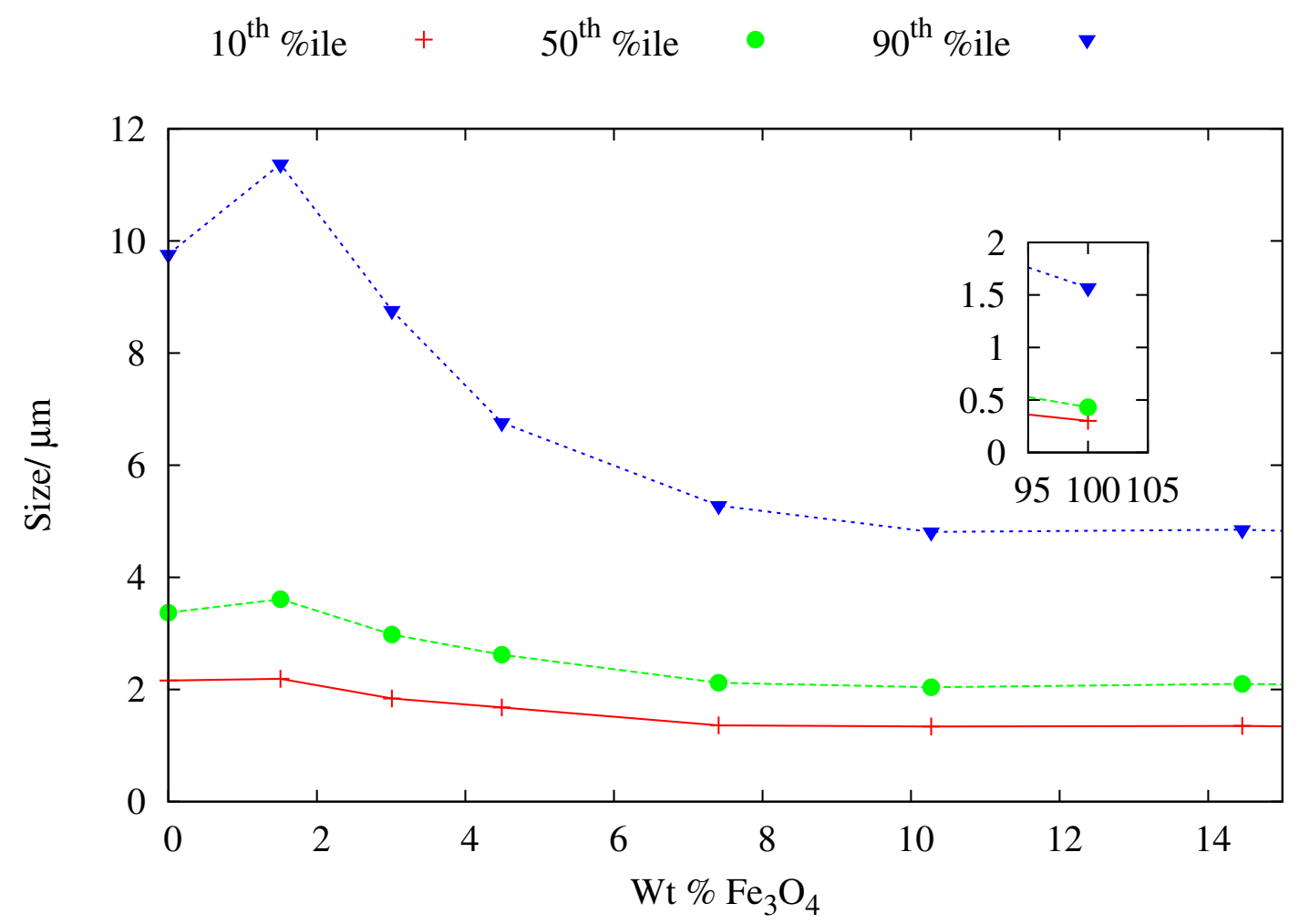

(b)

Figure 4.5 Particle size distribution of $\mathrm{Fe}_{3} \mathrm{O}_{4}$-NCS slurries prepared with nanosized superparamagnetic $\mathrm{Fe}_{3} \mathrm{O}_{4}$. Data sets in (a) are listed as weight percentage $\mathrm{Fe}_{3} \mathrm{O}_{4}$. 
precursor calcium hydroxide and sodium silicate solutions were $[\mathrm{Ca}]=0.624 \mathrm{~mol} \mathrm{dm}^{-3}$ and $\left[\mathrm{SiO}_{2}\right]=0.572 \mathrm{~mol} \mathrm{dm}^{-3}$. The addition of magnetite into the NCS structure does not have a significant effect on the particle size of the resultant composite. As the magnetite content of the composite is increased, its particle size decreases. This would be expected should the magnetite be providing additional nucleation sites for the NCS. The additional nucleation sites would cause a increase in the rate of nucleation, meaning that a greater number of smaller particles would be formed from a given amount of reagents.

It should be noted that the recorded particle size distribution for the $100 \%$ magnetite sample is not truly representative of the actual particle size of the material. The magnetite suspension could pass cleanly through a $0.45 \mu \mathrm{m}$ filter membrane, the same size as the median particle size distribution. Agglomeration of the particles within the instrument would contribute to this apparent increase in particle size. The recorded particle size is also very close to the resolution of the Mastersizer instrument, of approximately $0.1 \mu \mathrm{m}$. For this reason, particle size of the $100 \%$ magnetite sample was estimated from XRD analysis.

\subsubsection{Surface Area}

Figure 4.6 shows the variation in surface area for the $\gamma-\mathrm{Fe}_{2} \mathrm{O}_{3}-\mathrm{NCS}$ composite materials. As expected, the 2-ethoxyethanol washed materials possess a significantly greater surface area than the water washed composites. The range of surface area is within that expected for a typical non-composite NCS $\left(150 \pm 50 \mathrm{~m}^{2} \mathrm{~g}^{-1}\right.$ for water washed materials and $500 \pm 100 \mathrm{~m}^{2} \mathrm{~g}^{-1}$ for the 2-ethoxyethanol materials). ${ }^{22}$ As the $\gamma-\mathrm{Fe}_{2} \mathrm{O}_{3}$ content of the composite materials increases, the surface area rapidly increases, then slowly decreases. The decrease in surface area with increasing $\gamma-\mathrm{Fe}_{2} \mathrm{O}_{3}$ content may be explained by considering that addition maghemite leads to an increase in the density of the NCS composite. As such, the specific surface area will decrease.

The observed surface area increase at low $\gamma-\mathrm{Fe}_{2} \mathrm{O}_{3}$ content has a less obvious origin. It is probable that the presence of the maghemite crystallites provides for additional nucleation sites during the synthesis of the NCS composite. This would lead to the formation of 
smaller NCS particulates, having a higher surface area to volume ratio, and hence a higher absolute surface area. At low maghemite content, the higher surface area to volume ratio would outweigh the diminutive effect of the increased mass on the specific surface area.

The increase in specific surface area at low $\gamma-\mathrm{Fe}_{2} \mathrm{O}_{3}$ content is consistent with the decrease in particle size observed for the precursor $\mathrm{Fe}_{3} \mathrm{O}_{4}$-NCS materials observed in Sections 4.2.1.1 and 4.2.2.1.

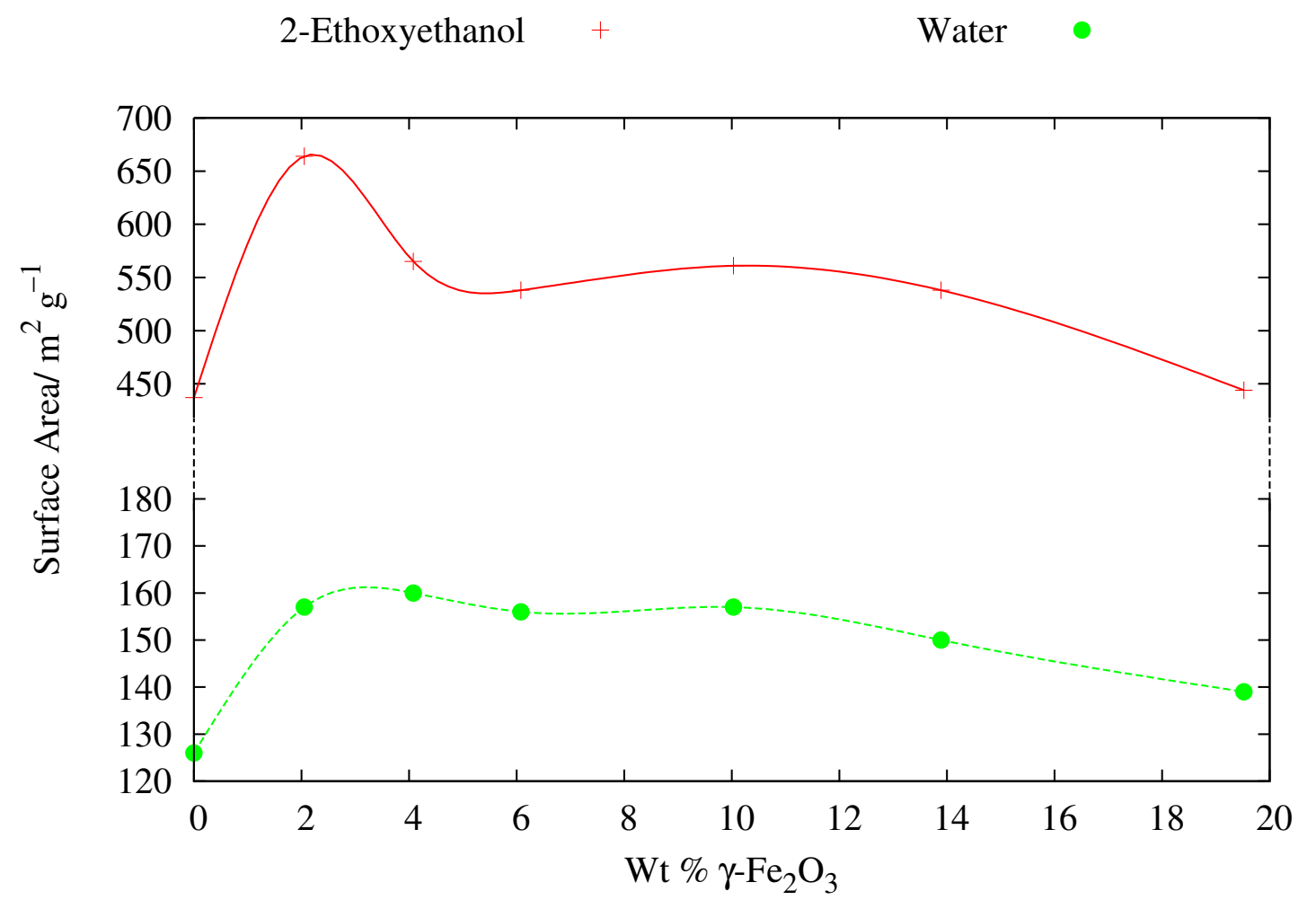

Figure 4.6 Surface area of $\gamma$ - $\mathrm{Fe}_{2} \mathrm{O}_{3}$-NCS composite materials, with 2-ethoxyethanol and water washed material shown.

\subsubsection{X-Ray Diffraction}

The particle size of the superparamagnetic magnetite suspension was estimated using the Scherrer formula, relating the X-ray diffraction linewidth to size of the crystalline domains within a particle: ${ }^{105}$

$$
t=\frac{0.9 \lambda}{B \cos (\theta)}
$$

in which $t$ is the particle diameter, $\lambda$ is the wavelength of incident $\mathrm{X}$-ray radiation, and $B$ is the thickness of the diffraction peak centred at an angle $\theta$. Figure 4.7 shows the diffraction pattern of the superparamagnetic $\mathrm{Fe}_{3} \mathrm{O}_{4}$. The four most intense diffraction peaks assigned 
to magnetite have been modelled with Gaussian functions (given in Equation (4.11), where $h$ is the peak height, $c$ its centre and $w$ its half width at half median height). Parameters of these functions are given in Table 4.2. Analysis of the four most intense diffraction peaks indicates a particle diameter in the range of $13-15 \mathrm{~nm}$. This is within the reported size range for magnetite particles synthesised in a similar manner to the synthesis methods used here. ${ }^{45,46}$ This particle diameter is also below the bottom end of the measurement range for the particle size analyser, explaining the discrepancy observed in the particle size measurement by this method.

$$
f(x)=h \exp \left(-\ln (2)\left(\frac{x-c}{w}\right)^{2}\right)
$$

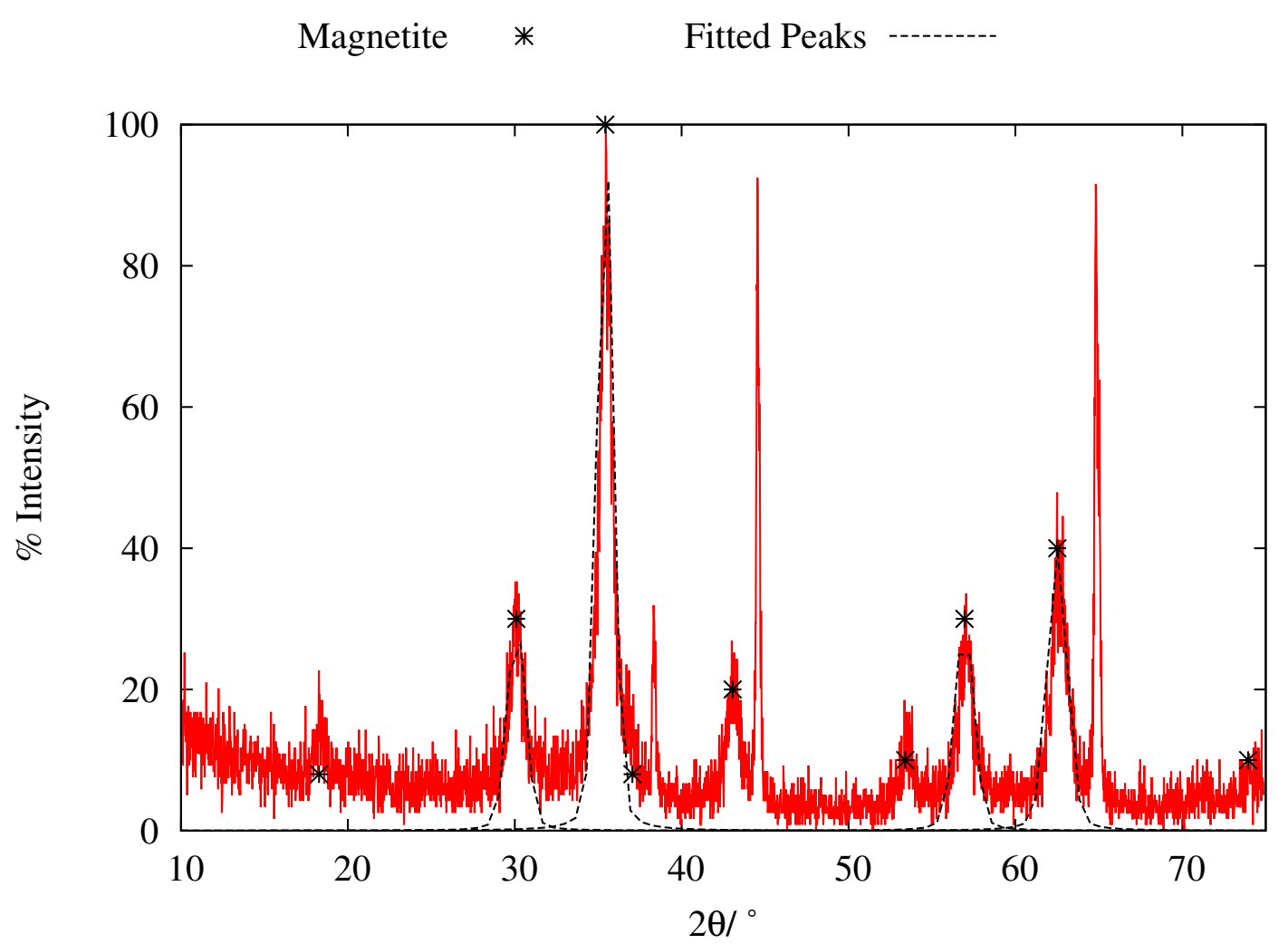

Figure 4.7 X-ray diffraction pattern of superparamagnetic $\mathrm{Fe}_{3} \mathrm{O}_{4}$.

Figure 4.8 shows the result of drying the superparamagnetic $\mathrm{Fe}_{3} \mathrm{O}_{4}$-NCS composite materials at $110^{\circ} \mathrm{C}$. Although exhibiting diffractions consistent with maghemite, $\gamma$ $\mathrm{Fe}_{2} \mathrm{O}_{3}$, analysis of the XRD pattern cannot be used to unambiguously identify the crystal phase as $\gamma-\mathrm{Fe}_{2} \mathrm{O}_{3}$ or $\mathrm{Fe}_{3} \mathrm{O}_{4}$, which exhibit very similar diffraction patterns. In this case, the assignment of $\gamma-\mathrm{Fe}_{2} \mathrm{O}_{3}$ as the predominant crystalline iron phase (instead of $\mathrm{Fe}_{3} \mathrm{O}_{4}$ ) was due to a colour change in the material from grey/black to ochre. The unassigned 
peaks in the XRD pattern are diffractions arising as a result of the NCS material itself (see Figure 3.11). As with Figure 4.7, the fitted maghemite peak was used to estimate the size of the crystallites. The peak is centred at $35.69^{\circ} 2 \theta$ with a half width, HWHM, of $0.46^{\circ} 2 \theta$. From Equation 4.10, a particle diameter of $21 \mathrm{~nm}$ may be calculated. This is 40\% larger than the particle size calculation provided in Table 4.2. An increase in particle size upon oxidation is to be expected. Both $\gamma-\mathrm{Fe}_{2} \mathrm{O}_{3}$ and $\mathrm{Fe}_{3} \mathrm{O}_{4}$ have the inverse spinel structure, ${ }^{41}$ with the former exhibiting iron vacancies. ${ }^{105}$ The particles are still within the size range expected for superparamagnetic behaviour.

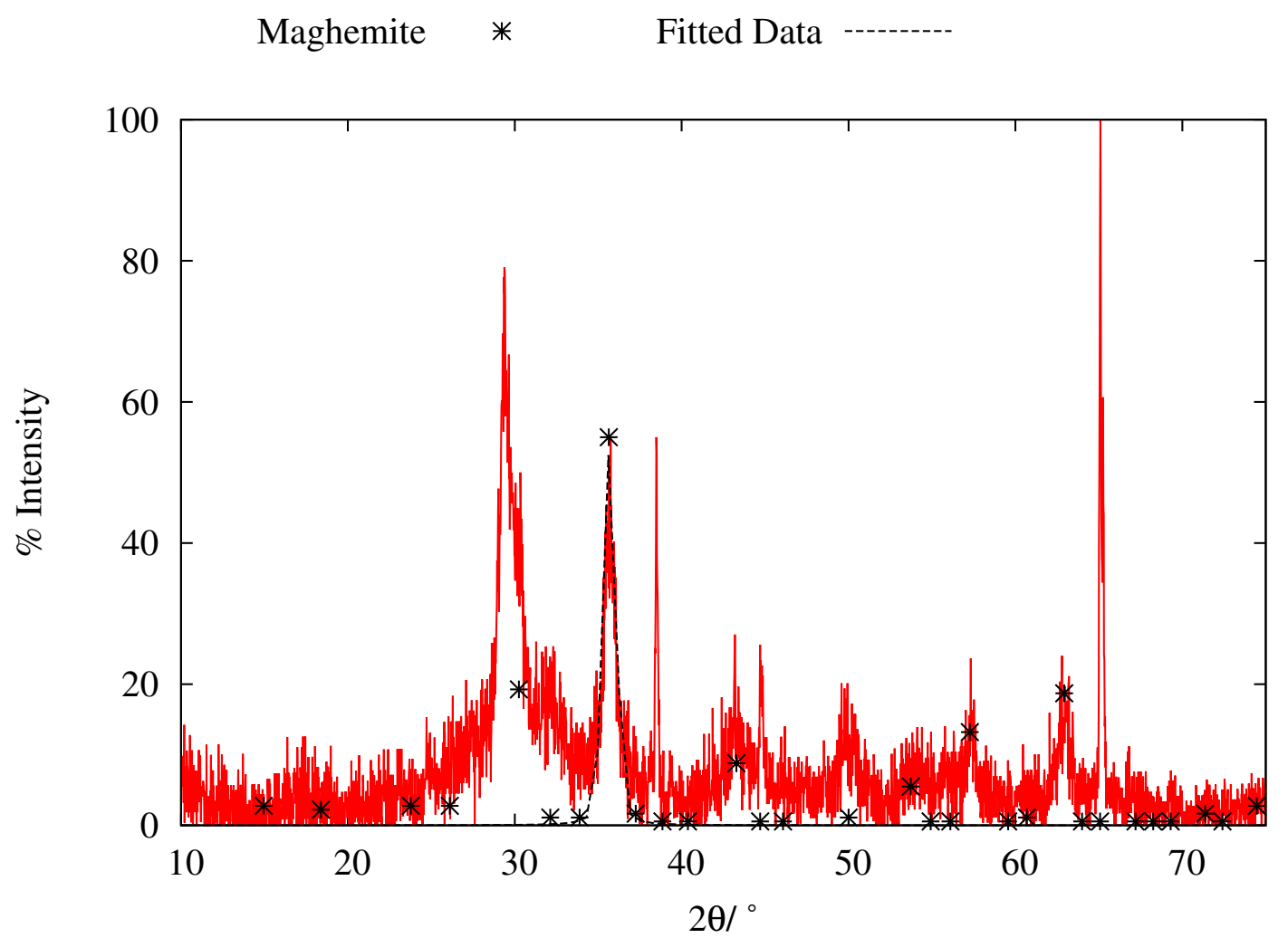

Figure 4.8 X-ray diffraction pattern of superparamagnetic $\mathrm{Fe}_{3} \mathrm{O}_{4}$-NCS composite air dried at $110^{\circ} \mathrm{C}$, showing conversion of $\mathrm{Fe}_{3} \mathrm{O}_{4}$ to a $\gamma-\mathrm{Fe}_{2} \mathrm{O}_{3}$ (maghemite) phase.

Table 4.2 Parameters for X-ray diffraction pattern shown in Figure 4.7.

\begin{tabular}{|c|c|c|c|c|}
\hline \multicolumn{2}{|r|}{ Centre $^{a}$} & \multicolumn{2}{|c|}{$\mathbf{H W H M}^{b}$} & \multirow{2}{*}{ Crystal diameter/ nm } \\
\hline$\rho^{\circ} 2 \theta$ & d-spacing/ Å & $/^{\circ} 2 \theta$ & / rad & \\
\hline 35.42 & 2.53 & 0.55 & $9.6 \times 10^{-3}$ & 15 \\
\hline 62.51 & 1.48 & 0.70 & $1.3 \times 10^{-2}$ & 13 \\
\hline 56.94 & 1.62 & 0.66 & $1.1 \times 10^{-2}$ & 14 \\
\hline 30.09 & 2.97 & 0.63 & $1.1 \times 10^{-2}$ & 13 \\
\hline
\end{tabular}

${ }^{a} \overline{\text { Peaks in order of decreasing intensity. }}$

${ }^{b}$ Half peak width at median height. 


\subsubsection{X-Ray Photoelectron Spectroscopy}

Figure 4.9 shows the XPS analysis of a superparamagnetic $\gamma-\mathrm{Fe}_{2} \mathrm{O}_{3}-\mathrm{NCS}$ composite material, containing 14.5 wt $\% \mathrm{Fe}_{3} \mathrm{O}_{4}$ prior to its oxidation to the $\gamma-\mathrm{Fe}_{2} \mathrm{O}_{3}$ phase. Parameters for the modelled peaks are given in Table 4.3. It can be seen from the figure that no signal was observed for iron. The XPS detects electrons ejected from the sample via an X-ray beam. As such, it is a surface-only analytical technique; any electrons ejected from sub-surface atoms are absorbed by shallower atoms before they reach the detector. Thus, the lack of an iron signal indicates that the iron (and hence $\gamma-\mathrm{Fe}_{2} \mathrm{O}_{3}$ ) is not present on the sample surface. Parameters for the $\mathrm{Ca}, \mathrm{O}$ and Si fitted peaks are otherwise very similar to those observed in unmodified NCS. ${ }^{47}$

Table 4.3 Parameters for the XPS peaks modelled in Figure 4.9.

\begin{tabular}{lrrr}
\hline Orbital & Centre/ eV & HWHM/ eV & Area $^{a}$ \\
\hline $\mathrm{Ca} 2 \mathrm{p}_{\frac{1}{2}}$ & 350.8 & 0.80 & 87.7 \\
$\mathrm{Ca} 2 \mathrm{p}_{\frac{3}{2}}$ & 347.6 & 0.68 & 100.0 \\
$\mathrm{Ca} 2 \mathrm{p}_{\frac{3}{2}}$ & 346.9 & 0.59 & 80.5 \\
\hline $\mathrm{O} 1 \mathrm{~s}$ & 532.9 & 0.95 & 75.8 \\
$\mathrm{O} 1 \mathrm{~s}$ & 530.4 & 0.62 & 60.0 \\
$\mathrm{O} 1 \mathrm{~s}$ & 531.8 & 0.83 & 100.0 \\
\hline Si $2 \mathrm{p}_{\frac{1}{2}}$ & 103.3 & 0.84 & 100.0 \\
$\mathrm{Si} 2 \mathrm{p}_{\frac{1}{2}}$ & 102.4 & 0.61 & 91.4 \\
$\mathrm{Si} 2 \mathrm{p}_{\frac{1}{2}}$ & 101.7 & 0.56 & 71.8 \\
\hline
\end{tabular}

${ }^{a}$ Normalised based on the area of the largest peak.

\subsubsection{Magnetic Hysteresis}

Figure 4.10 shows the saturation magnetic moment for a series of 2-ethoxyethanol washed $\gamma$ - $\mathrm{Fe}_{2} \mathrm{O}_{3}$ composites. NCS itself, at 0 wt $\% \gamma-\mathrm{Fe}_{2} \mathrm{O}_{3}$, is diamagnetic, meaning that the sample exhibits a negative magnetism relative to an applied field. At the magnetic field strengths used during these measurements, diamagnetic materials do not achieve magnetic saturation. The modelled line in the figure is linear, having an $R^{2}$ value of 0.993 . When this fitted line is extended to a $\gamma-\mathrm{Fe}_{2} \mathrm{O}_{3}$ content of $100 \mathrm{wt} \%$, a magnetic moment of $\sigma_{s}=33.9 \mathrm{~A} \mathrm{~m}^{2} \mathrm{~kg}^{-1}$ is predicted. This predicted value is significantly less that the actual 


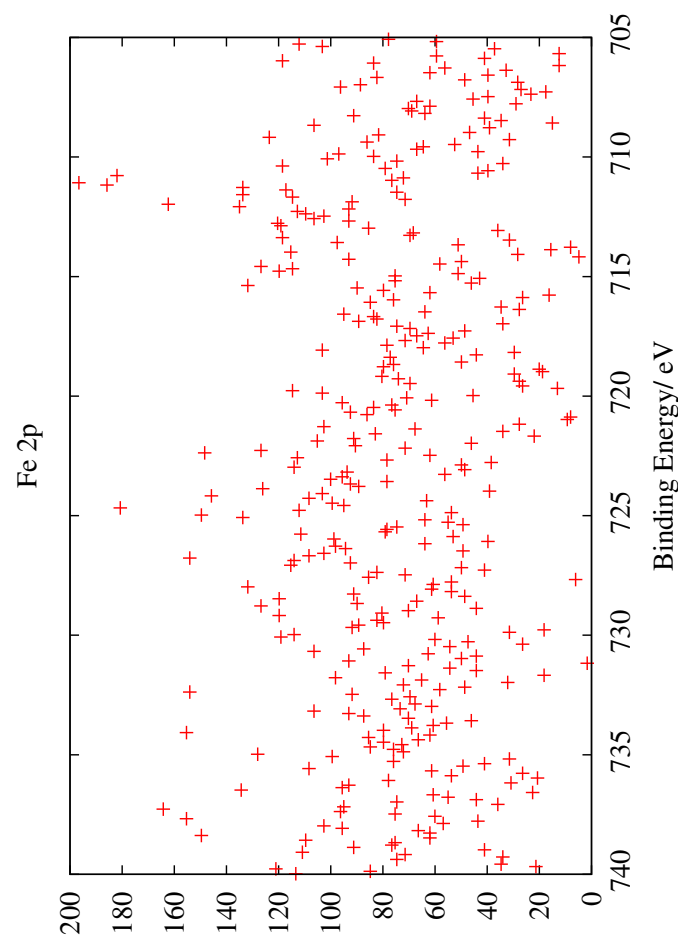

I- s słuno / אł̣!suəıฺU

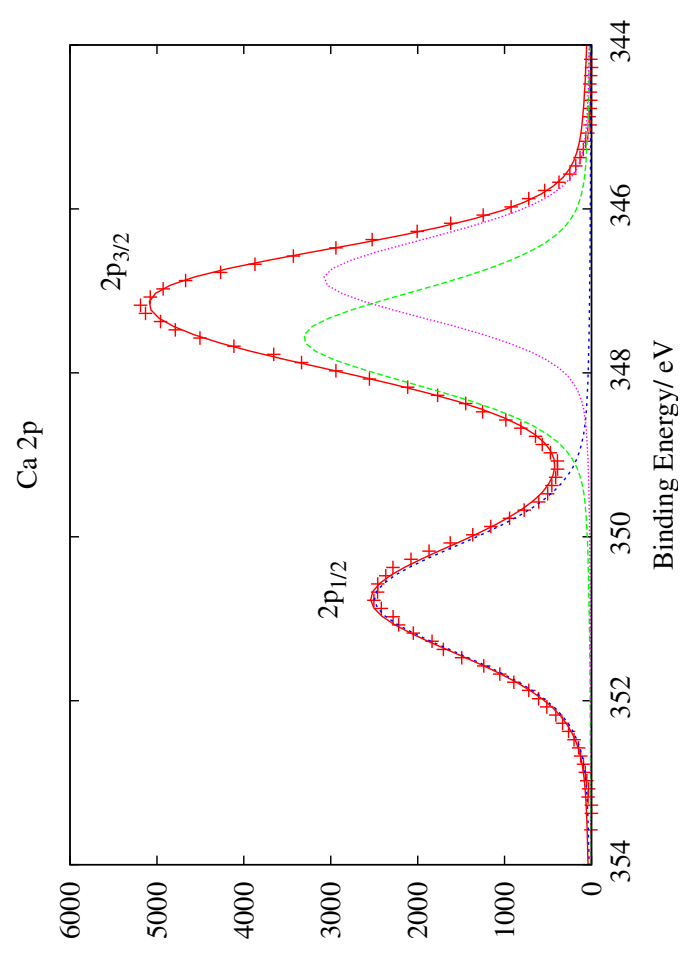

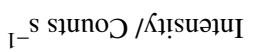

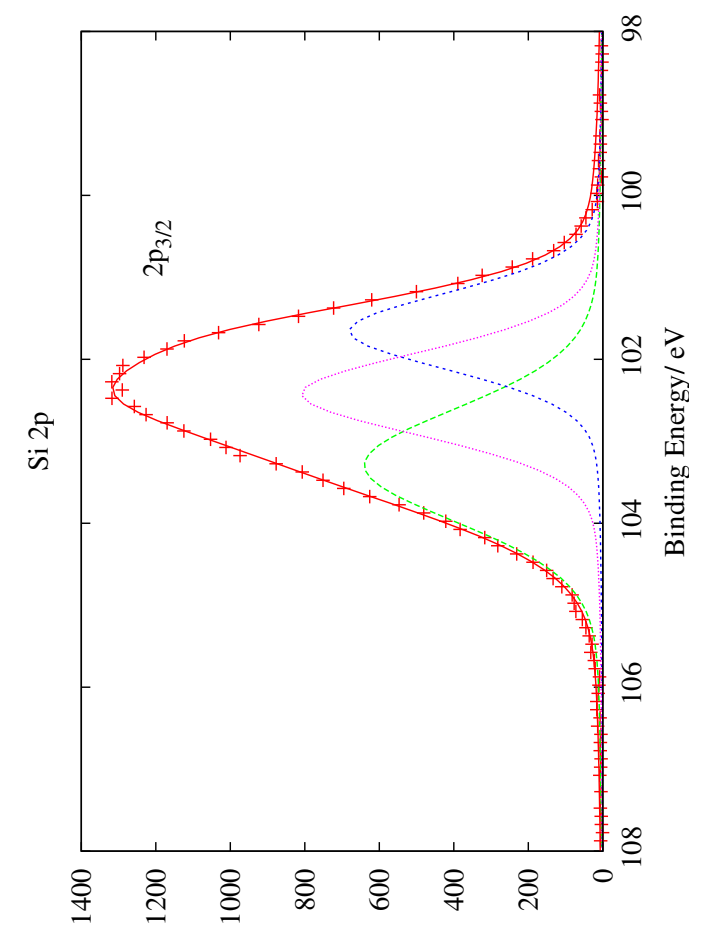

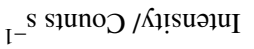

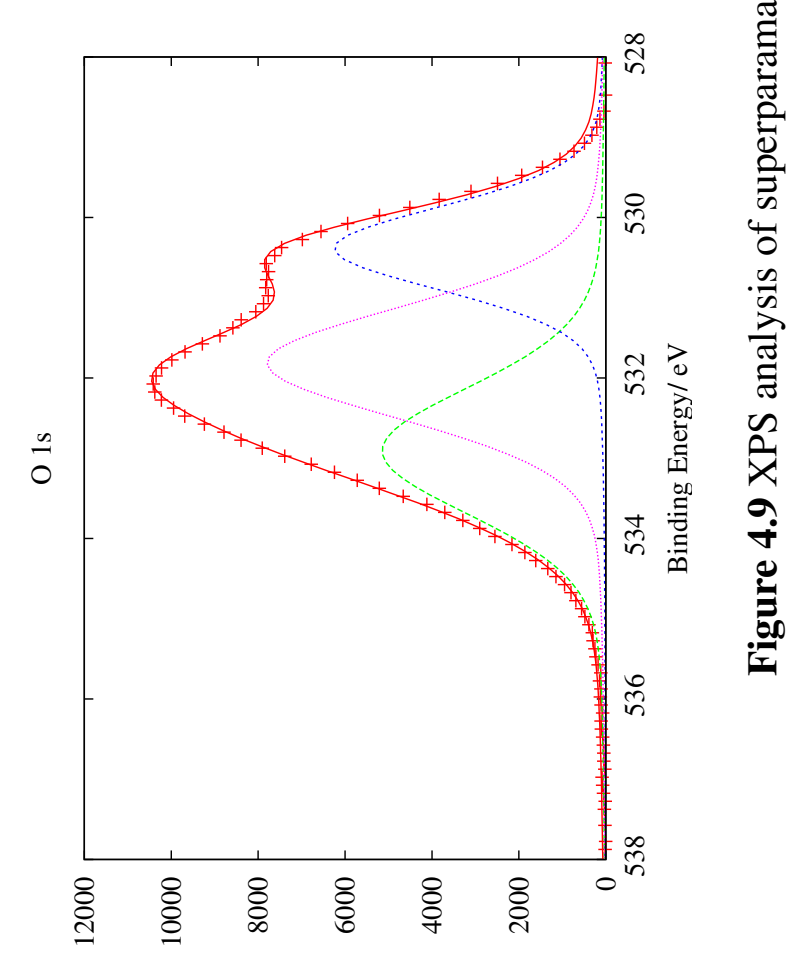

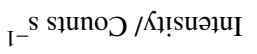


saturation magnetic moment for maghemite, $\sigma_{s}=75 \mathrm{~A} \mathrm{~m}^{2} \mathrm{~kg}^{-1} .{ }^{105}$ While the presence of diamagnetic NCS within the sample could contribute somewhat to the lower than expected saturation magnetisation, it is more likely that the iron-containing species is not present as a pure phase. As the $\gamma-\mathrm{Fe}_{2} \mathrm{O}_{3}$-NCS materials were formed via the oxidation of $\mathrm{Fe}_{3} \mathrm{O}_{4}$ NCS composites, it is possible that other iron oxide phases may have formed, especially hematite, $\alpha-\mathrm{Fe}_{2} \mathrm{O}_{3}$. In its bulk state, hematite is thermodynamically preferred with respect to maghemite $\left(\Delta \mathrm{H}=15 \mathrm{~kJ} \mathrm{~mol}^{-1}\right) .{ }^{118}$ However, hematite has a higher surface energy than maghemite. Therefore, for small particle sizes (higher surface areas) maghemite becomes the thermodynamically preferred phase. This has been reported to occur for particles with a diameter less than $15 \mathrm{~nm} .{ }^{118}$ During the oxidation from $\mathrm{Fe}_{3} \mathrm{O}_{4}$ to the iron (III) oxides, an increase in the particle diameter from $13-15 \mathrm{~nm}$ to $21 \mathrm{~nm}$ was observed. As this spans the boundary of the hematite/maghemite phase stability, it is possible that a mixture of the antiferrimagnetic $\alpha-\mathrm{Fe}_{2} \mathrm{O}_{3}$ and the ferrimagnetic $\gamma-\mathrm{Fe}_{2} \mathrm{O}_{3}$ was formed. The presence of some antiferrimagnetic material would account for the lower than expected saturation magnetic moment.

As made $\quad+\quad \quad$ After $\mathrm{Cu}^{2+}$ sorption

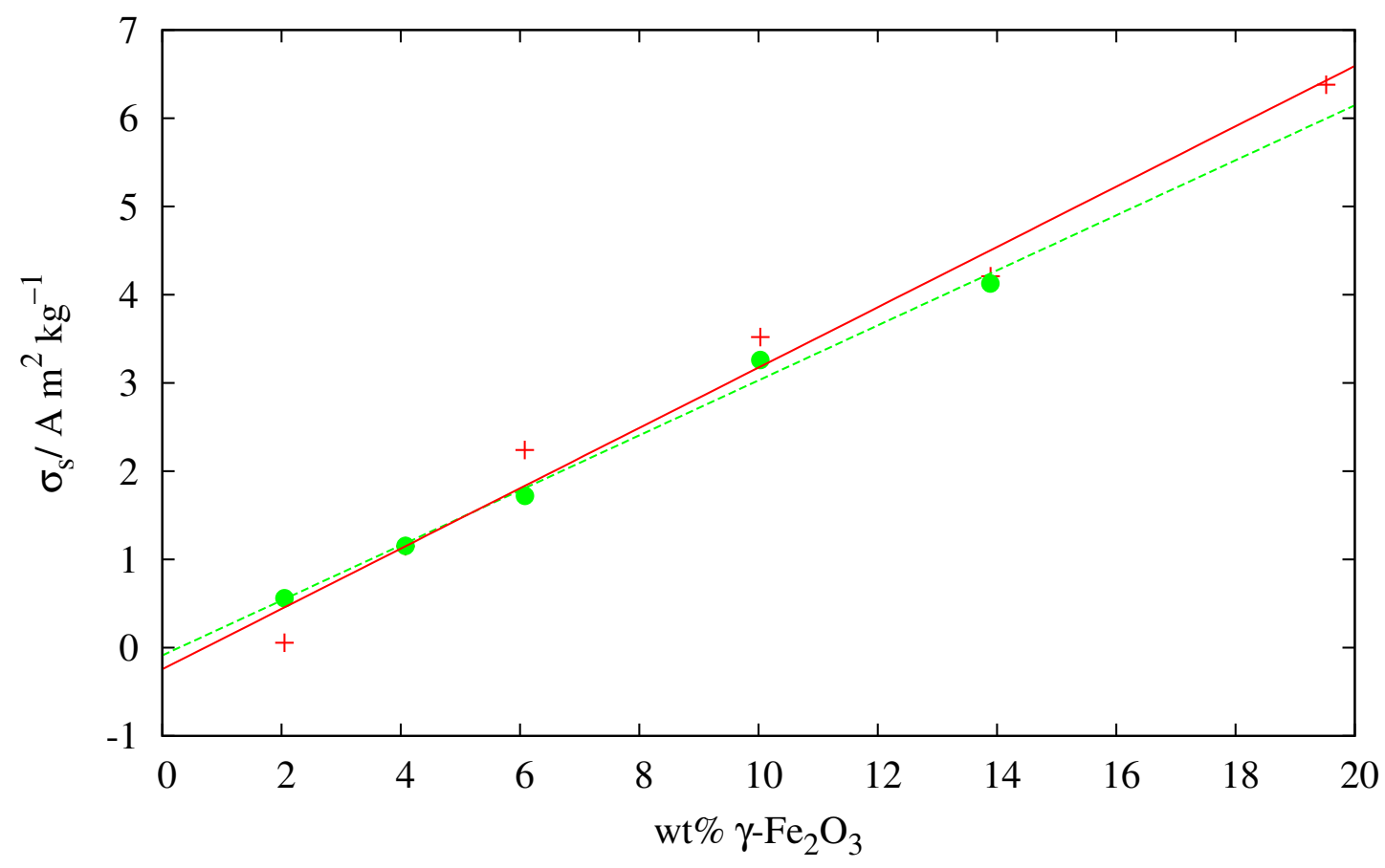

Figure 4.10 Saturation magnetic moment for superparamagnetic $\gamma-\mathrm{Fe}_{2} \mathrm{O}_{3}-\mathrm{NCS}$ composites, prior and subsequent to copper sorption.

Figure 4.10 also shows the effect of sorption of $\mathrm{Cu}^{2+}$ from a $7.9 \mathrm{mmol} \mathrm{dm}{ }^{-3} \mathrm{CuCl}_{2}$ 
solution on the saturation magnetic moment. It can be seen that the magnetic moment becomes slightly altered on copper sorption. The saturation magnetic moment also exhibits a linear relationship to the $\gamma-\mathrm{Fe}_{2} \mathrm{O}_{3}$ content, having an $R^{2}$ value of 0.998 . The plot in Figure 4.11 demonstrates that the material remains superparamagnetic; the remnant magnetic moments for these materials are approximately $1 \%$ that of the saturation magnetic moment.

\subsubsection{Electron Microscopy}

Figure 4.12 shows an EDS overlay map of a $\gamma-\mathrm{Fe}_{2} \mathrm{O}_{3}-\mathrm{NCS}$ composite material containing 14.5 wt $\% \gamma-\mathrm{Fe}_{2} \mathrm{O}_{3}$, with electron micrographs of the same sample shown in Figure 4.13. In the EDS overlay, silicon is represented by the colour red, calcium by green, and iron by blue. It can be seen that the NCS particle occupying the centre area of the image exhibits an even distribution of iron within it, as it appears white in the overlay. The particles to the edges of the image exhibit no iron content, so appear yellow due to their calcium and silicon content. This apparent lack of iron content may be attributed to the fact that these

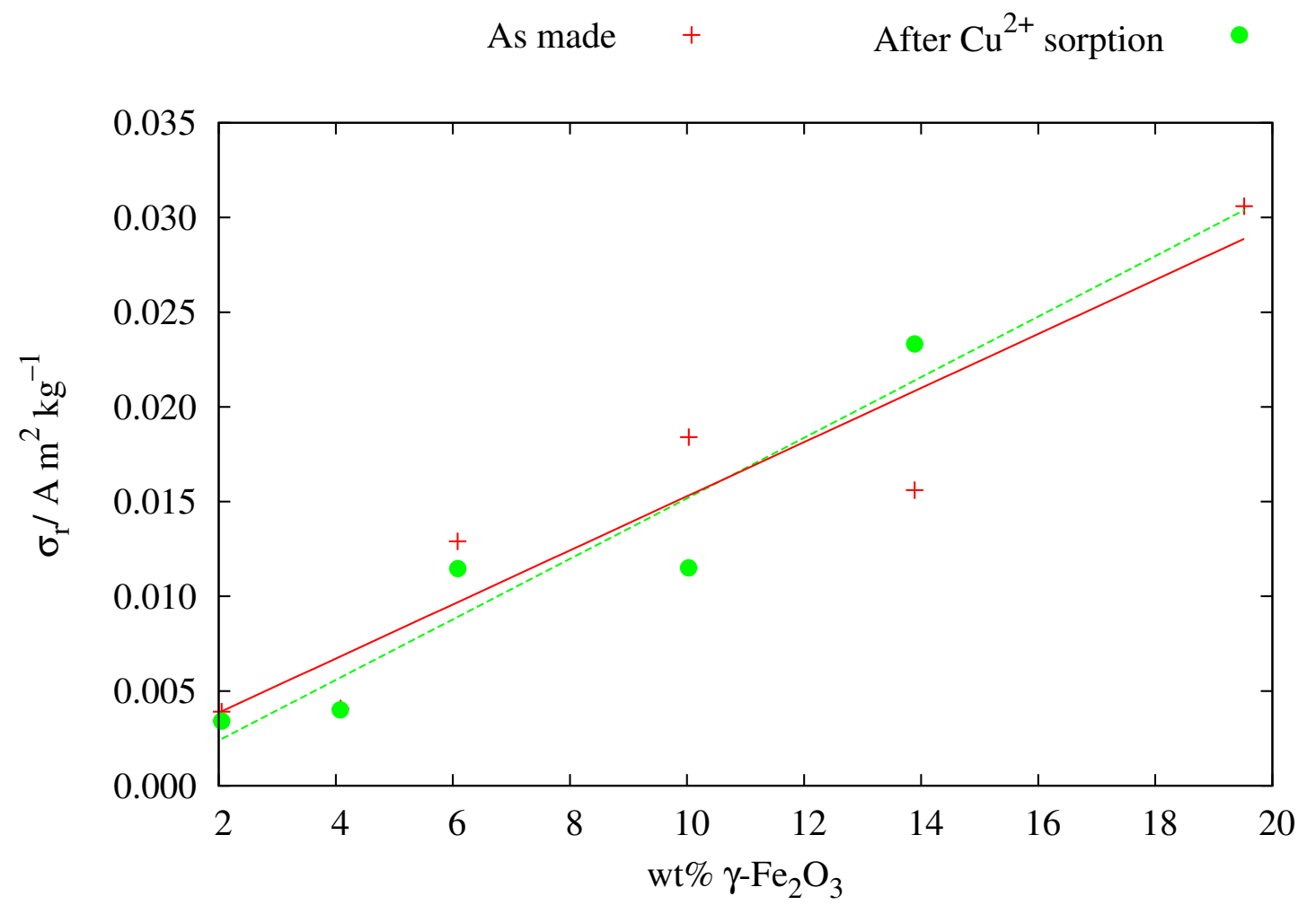

Figure 4.11 Remnant magnetic moment for $\gamma-\mathrm{Fe}_{2} \mathrm{O}_{3}$-NCS composites, prior and subsequent to copper sorption, demonstrating superparamagnetism in all samples. 
particles lie outside the focal plane of the instrument. This can be seen more clearly when examining the SEM image of the same area. When comparing the SEM image to the EDS overlay, it may be observed that the iron-containing section is much brighter than the other regions of the image. This arises due to the particle accumulating a higher charge, as it cannot discharge this excess charge to the sample mount, which is comparatively further from the sample.

Figure 4.13, showing increasing magnification of the maghemite-containing NCS particle shows no definitive evidence for the presence of surface bound $\gamma-\mathrm{Fe}_{2} \mathrm{O}_{3}$. At the highest magnification (50000 times), the scale bar represents a distance of $100 \mathrm{~nm}$. From this, it may be deduced that the individual NCS platelets are approximately 5-15 nm thick. This size range is similar to the calculated diameter of the $\gamma-\mathrm{Fe}_{2} \mathrm{O}_{3}$ particles of $21 \mathrm{~nm}$; with the precursor $\mathrm{Fe}_{3} \mathrm{O}_{4}$ phase having a diameter of $13-15 \mathrm{~nm}$. The lack of evidence for surface-bound $\gamma-\mathrm{Fe}_{2} \mathrm{O}_{3}$ in the electron microscopy images is consistent with the absence of an iron signal in the XPS studies, discussed previously. 

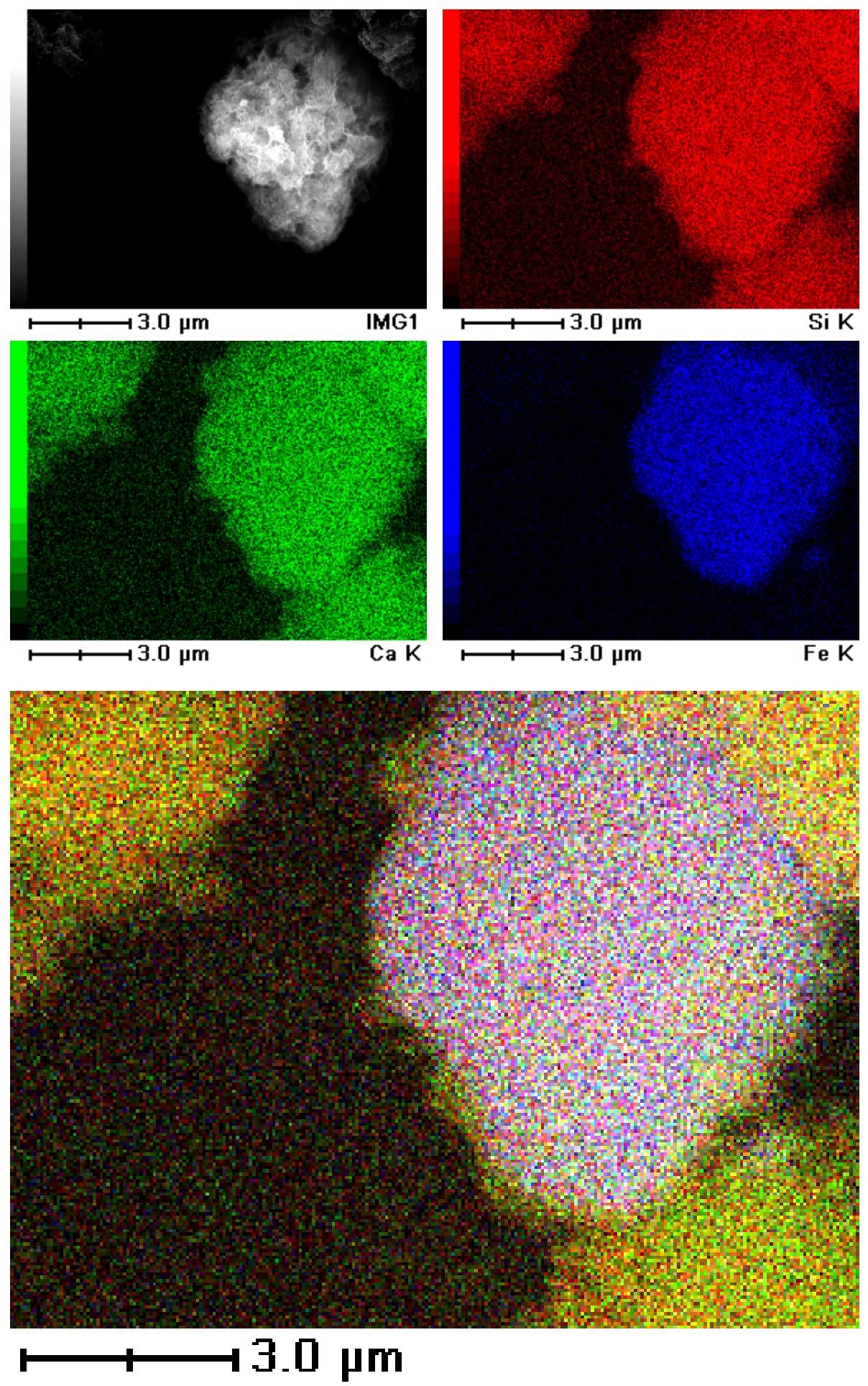

Figure 4.12 EDS overlay of 2-ethoxyethanol washed $\gamma-\mathrm{Fe}_{2} \mathrm{O}_{3}-\mathrm{NCS}$ prepared at 14.5 wt $\% \gamma-\mathrm{Fe}_{2} \mathrm{O}_{3}: \mathrm{Si}$ (red); $\mathrm{Ca}$ (green); Fe (blue). The SEM image may be seen in Figure 4.13. 

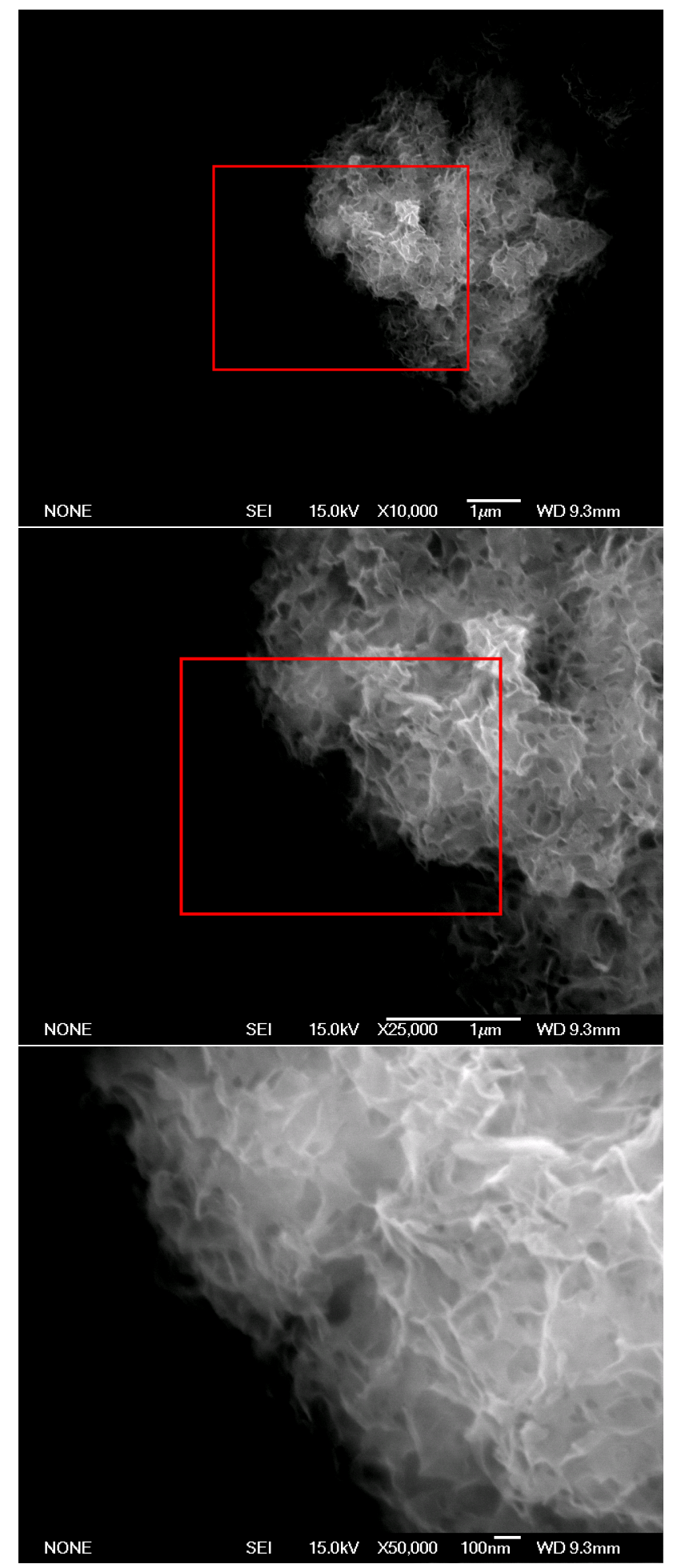

Figure 4.13 Electron micrographs of $\gamma-\mathrm{Fe}_{2} \mathrm{O}_{3}-\mathrm{NCS}$ at increasing magnification. 


\subsubsection{Magnetic Separation}

Based on Figure 4.10, it would appear that sorption of copper does not significantly alter the magnetic characteristics of the $\gamma$ - $\mathrm{Fe}_{2} \mathrm{O}_{3}$-NCS composite material; the saturation magnetic moment is only slightly altered as a result of copper sorption. By its nature however, magnetic moment measurements are made on the bulk material, and as such do not fully represent the nature of the material in contact with copper. An example of this can be seen in Figure 4.14. Figures 4.14(a) and (b) and show the effect of presence and absence of a magnetic field on a suspension of a $\gamma-\mathrm{Fe}_{2} \mathrm{O}_{3}-\mathrm{NCS}$ composite material. Figure 4.14(a) shows the effect of a magnetic field on the material following sorption of copper from a $7.9 \mathrm{mmol} \mathrm{dm}{ }^{-3} \mathrm{CuCl}_{2}$ solution. Following copper sorption, the material is not cleanly separated by a magnetic field; a significant amount of material remains in suspension. This remnant material in suspension is most likely generated due to the acidic nature of the copper solution, causing dissociation of $\gamma-\mathrm{Fe}_{2} \mathrm{O}_{3}$ from the NCS material.

Given that the $\gamma-\mathrm{Fe}_{2} \mathrm{O}_{3}$-NCS material did not maintain its integrity under the acidic conditions of the copper sorption studies, further investigation of magnetic separation techniques as applied to this material were not undertaken. Instead, $\mathrm{Fe}_{3} \mathrm{O}_{4}$-NCS materials were studied. 


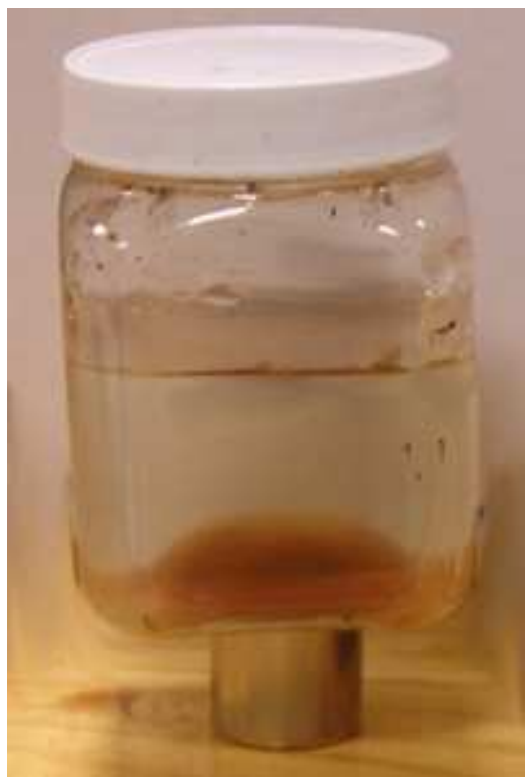

(a)

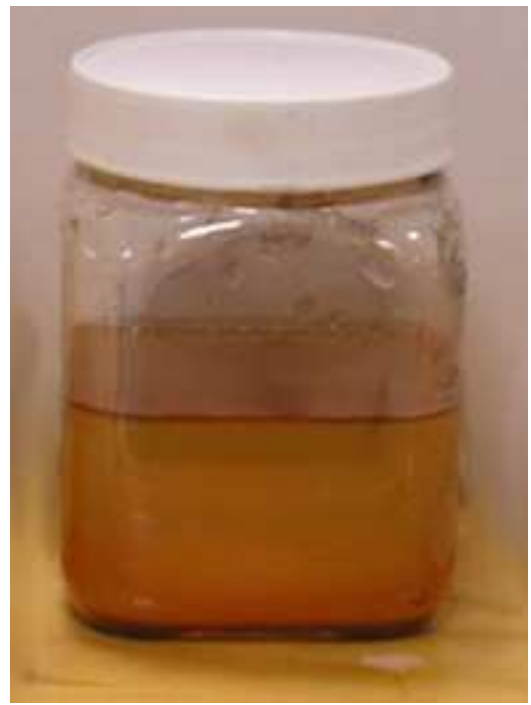

(b)

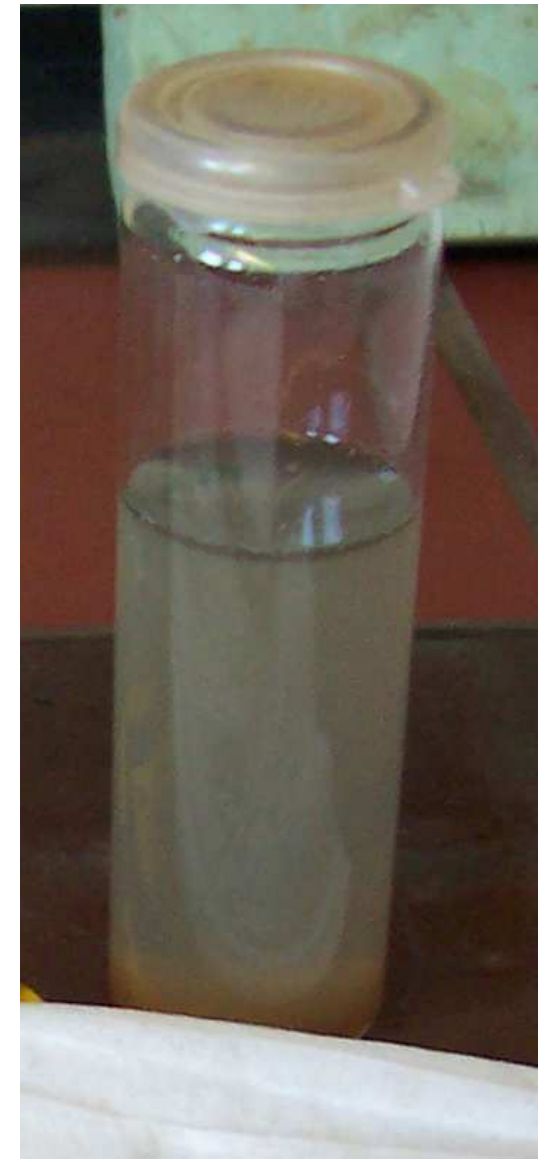

(c)

Figure 4.14 Magnetic separation of $\gamma$ - $\mathrm{Fe}_{2} \mathrm{O}_{3}$-NCS composites: prior to copper sorption (a) in the presence and (b) absence of magnetic field; (c) after copper sorption in the presence of magnetic field. 


\subsubsection{Bulk Magnetite-NCS Composites}

\subsubsection{Particle Size Analysis}

Figure 4.15 shows the particle size distribution for a series of $\mathrm{Fe}_{3} \mathrm{O}_{4}$-NCS composite materials prepared from high concentration precursor calcium hydroxide and silicate solutions $\left([\mathrm{Ca}]=0.937 \mathrm{~mol} \mathrm{dm}^{-3},\left[\mathrm{SiO}_{2}\right]=0.858 \mathrm{~mol} \mathrm{dm}^{-3}\right)$ with the addition of magnetite powder. The effect of bulk-magnetite addition of the particle size distribution is slightly different to that observed with the addition of the superparamagnetic $\mathrm{Fe}_{3} \mathrm{O}_{4}$ (Figure 4.5). Addition of bulk magnetite to the NCS results is a slightly broader particle size distribution. Although the particle size distribution does not exhibit a classic bellshaped curve, a bimodal size distribution is not evident. This indicates that the magnetite and NCS do not exist as separate particles. Increasing the magnetite content from 10 to $15 \mathrm{wt} \%$ results in a sudden decrease in the particle size, whereas a smoother trend was observed in the size distribution of the $\gamma-\mathrm{Fe}_{2} \mathrm{O}_{3}$-NCS composites. It is possible that the change in recorded particle size is due to the operating conditions of the instrument.

The Malvern Mastersizer instrument determines particle size based on the scattering of laser light. As such, the absorption coefficient of the material will significantly alter the observed particle size measurement. Samples having a higher absorbance scatter less light, meaning they will appear as a lower concentration to the instrument (compared to samples having a lower absorbance). Figure 4.16 and Table 4.4 show the effects of altering the absorption coefficient on recorded particle size distribution. The chosen absorption coefficients represent the standard software settings for the nearest equivalent materials $\mathrm{CaSiO}_{3}(0.1)$ and iron oxide (1.0). It can be seen from the figure that there is a significant difference in the recorded particle sizes. The measurement performed at the higher absorption coefficient resulted in recorded particle sizes $10 \%$ of those recorded at the lower absorption setting. Except for particle size measurement on pure $\mathrm{Fe}_{3} \mathrm{O}_{4}$, the Malvern instrument was set to measure particles having an absorbance of 0.1. As the absorbance of the $\mathrm{Fe}_{3} \mathrm{O}_{4}$-NCS composite materials increases with an increase in $\mathrm{Fe}_{3} \mathrm{O}_{4}$ content, it is likely that the recorded particle sizes were underestimated. 


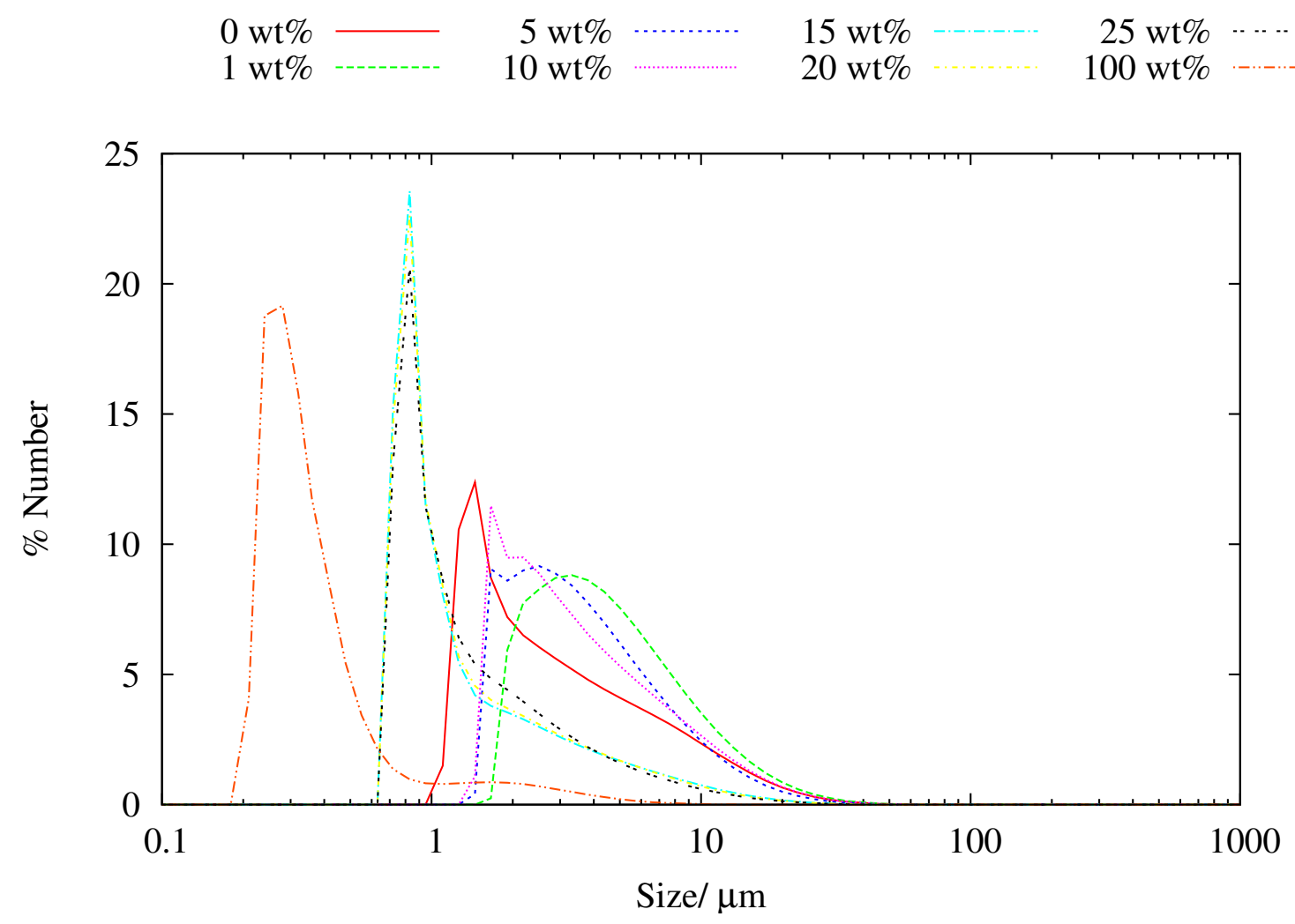

(a)

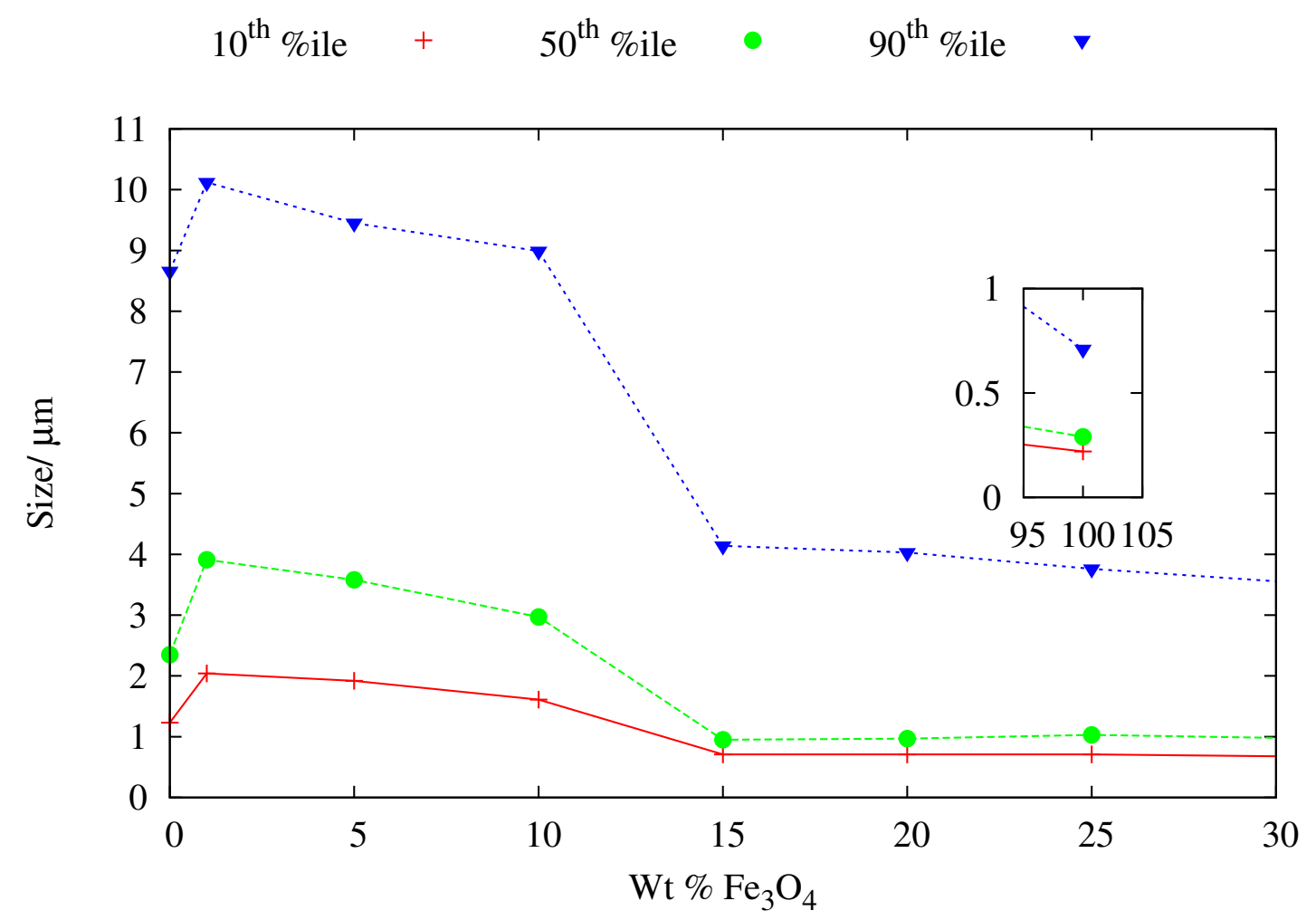

(b)

Figure 4.15 Particle size distribution of $\mathrm{Fe}_{3} \mathrm{O}_{4}$-NCS slurries prepared with bulk $\mathrm{Fe}_{3} \mathrm{O}_{4}$ powder. Data sets in (a) are listed as weight percentage $\mathrm{Fe}_{3} \mathrm{O}_{4}$. 
The smaller particle size distribution with increasing $\mathrm{Fe}_{3} \mathrm{O}_{4}$ content may be attributed to a faster initial precipitation rate. The presence of magnetite particles within the sodium silicate solution can provide additional nucleation sites for the precipitation of NCS. These additional sites would mean a greater number of smaller particles of NCS would form in the initial mixing step. This does not significantly reduce the particle size however. As can be shown below (Figure 4.5), the degree of mixing has a greater effect on the particle size distribution.

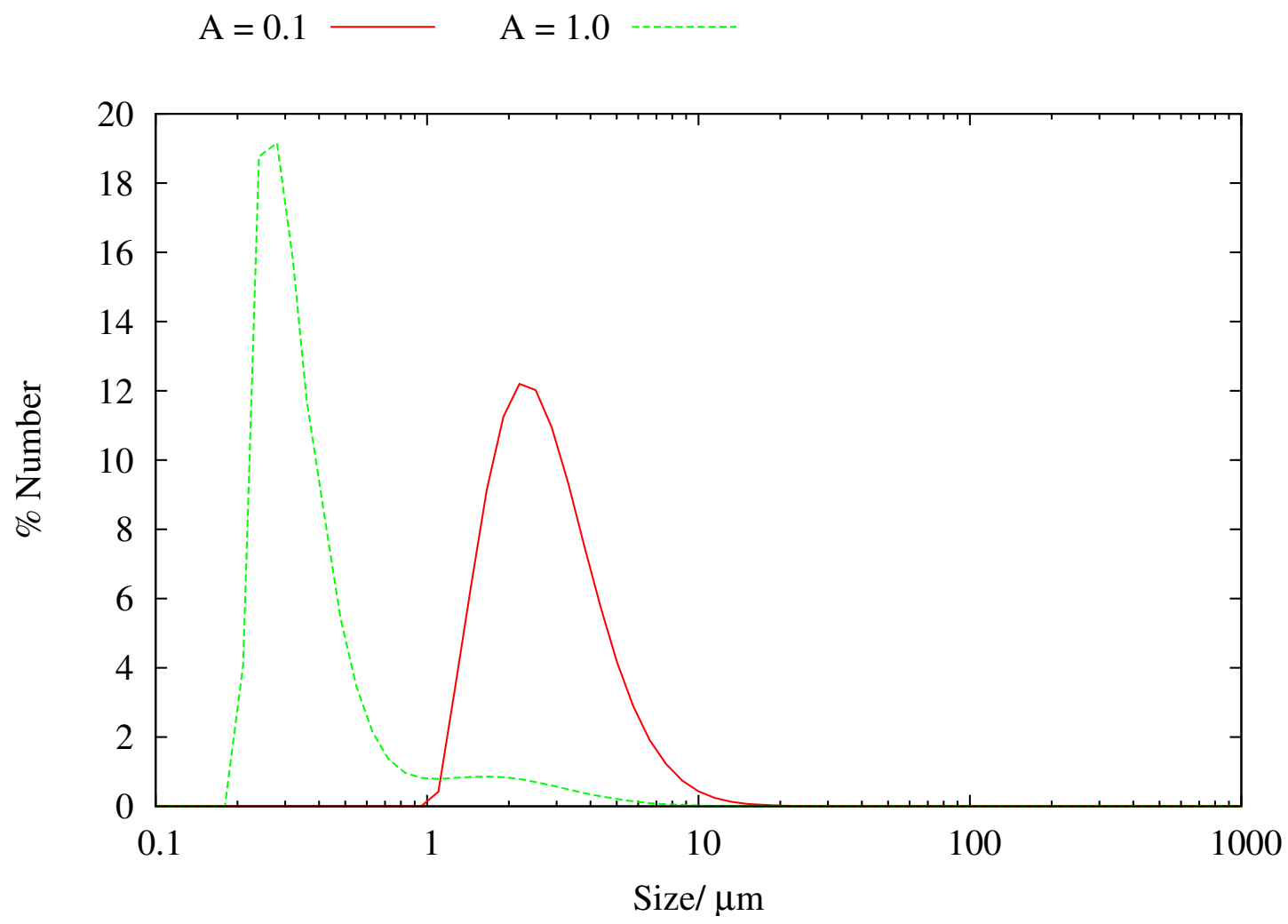

Figure 4.16 Effect of instrument absorbance setting on recorded particle size distribution of $\mathrm{Fe}_{3} \mathrm{O}_{4}$ powder.

Table 4.4 Effect of material absorbance on recorded particle size distribution of $\mathrm{Fe}_{3} \mathrm{O}_{4}$ powder.

\begin{tabular}{llr}
\hline \multirow{2}{*}{ Percentile } & \multicolumn{2}{l}{ Particle Size/ $\mu \mathbf{m}$} \\
\cline { 2 - 3 } & \multicolumn{2}{l}{ Absorbance Setting } \\
\cline { 2 - 3 } & \multicolumn{1}{c}{$\mathbf{0 . 1}$} & $\mathbf{1 . 0}$ \\
\hline $10^{\text {th }}$ & 1.44 & 0.22 \\
$50^{\text {th }}$ & 2.38 & 0.29 \\
$90^{\text {th }}$ & 4.62 & 0.71 \\
\hline
\end{tabular}

${ }^{a}$ Selected absorption coefficients are taken from standard operating conditions provided for $\mathrm{CaSiO}_{3}(0.1)$ and iron oxide (1.0) in Malvern Mastersizer 2000 software. 


\subsubsection{X-Ray Diffraction}

A typical X-ray diffraction pattern for the $\mathrm{Fe}_{3} \mathrm{O}_{4}$-NCS composite materials prepared with bulk $\mathrm{Fe}_{3} \mathrm{O}_{4}$ can be seen in Figure 4.17. It can be seen that $\mathrm{Fe}_{3} \mathrm{O}_{4}$ is the predominant crystalline phase present. The peaks indexed to the aluminium diffraction pattern arise from the sample holder employed for XRD measurements, and the nonindexed peaks are typical of NCS itself. The four most intense peaks attributable to the $\mathrm{Fe}_{3} \mathrm{O}_{4}$ pattern have been modeled by a Gaussian function, the parameters of which are shown in Table 4.5. These parameters have been used to derive the crystallite size from the Scherrer equation (4.10). While the calculated crystallite sizes (51-66 nm) are significantly larger than those for the superparamagnetic $\mathrm{Fe}_{3} \mathrm{O}_{4}$ species at 13-15 nm (Table 4.2), they are most likely underestimated. The underestimation arises, in part, as the XRD instrument has a finite resolution. This can be most easily seen in the modelled peak from the aluminium diffraction pattern. As this peak is caused by diffraction from the bulk aluminium metal of the sample holder, it may be expected that the width of this peak represents the resolution of the XRD instrument, in this case a peak half width of $0.10^{\circ} 2 \theta$. If this is taken as the instrumental resolution, it can be seen that the instrument resolution comprises approximately one half of the width of the $\mathrm{Fe}_{3} \mathrm{O}_{4}$ diffraction peaks. Thus, the degree of line broadening attributable to small particulate size is minimal.

Table 4.5 Parameters for X-ray diffraction pattern shown in Figure 4.17.

\begin{tabular}{|c|c|c|c|c|c|}
\hline \multicolumn{2}{|c|}{ Centre $^{a}$} & \multicolumn{2}{|c|}{ HWHM $^{b}$} & \multirow{2}{*}{$\begin{array}{c}\text { Crystal diameter } \\
\text { / nm }\end{array}$} & \multirow{2}{*}{ Phase } \\
\hline$\rho^{\circ} 2 \theta$ & d-spacing/ Å & $\rho^{\circ} 2 \theta$ & / rad & & \\
\hline 35.57 & 2.52 & 0.13 & $1.1 \times 10^{-3}$ & 66 & \\
\hline 62.73 & 1.48 & 0.17 & $1.5 \times 10^{-3}$ & 54 & \\
\hline 57.15 & 1.61 & 0.18 & $1.5 \times 10^{-3}$ & 51 & $\mathrm{e}_{3} \mathrm{O}_{4}$ \\
\hline 30.23 & 2.95 & 0.14 & $1.2 \times 10^{-3}$ & 59 & \\
\hline 25.81 & 3.45 & 0.10 & $9.0 \times 10^{-4}$ & 79 & $\mathrm{Al}$ \\
\hline
\end{tabular}

${ }^{a} \overline{\text { Peaks in order of decreasing intensity. }}$

${ }^{b}$ Half peak width at median height. 


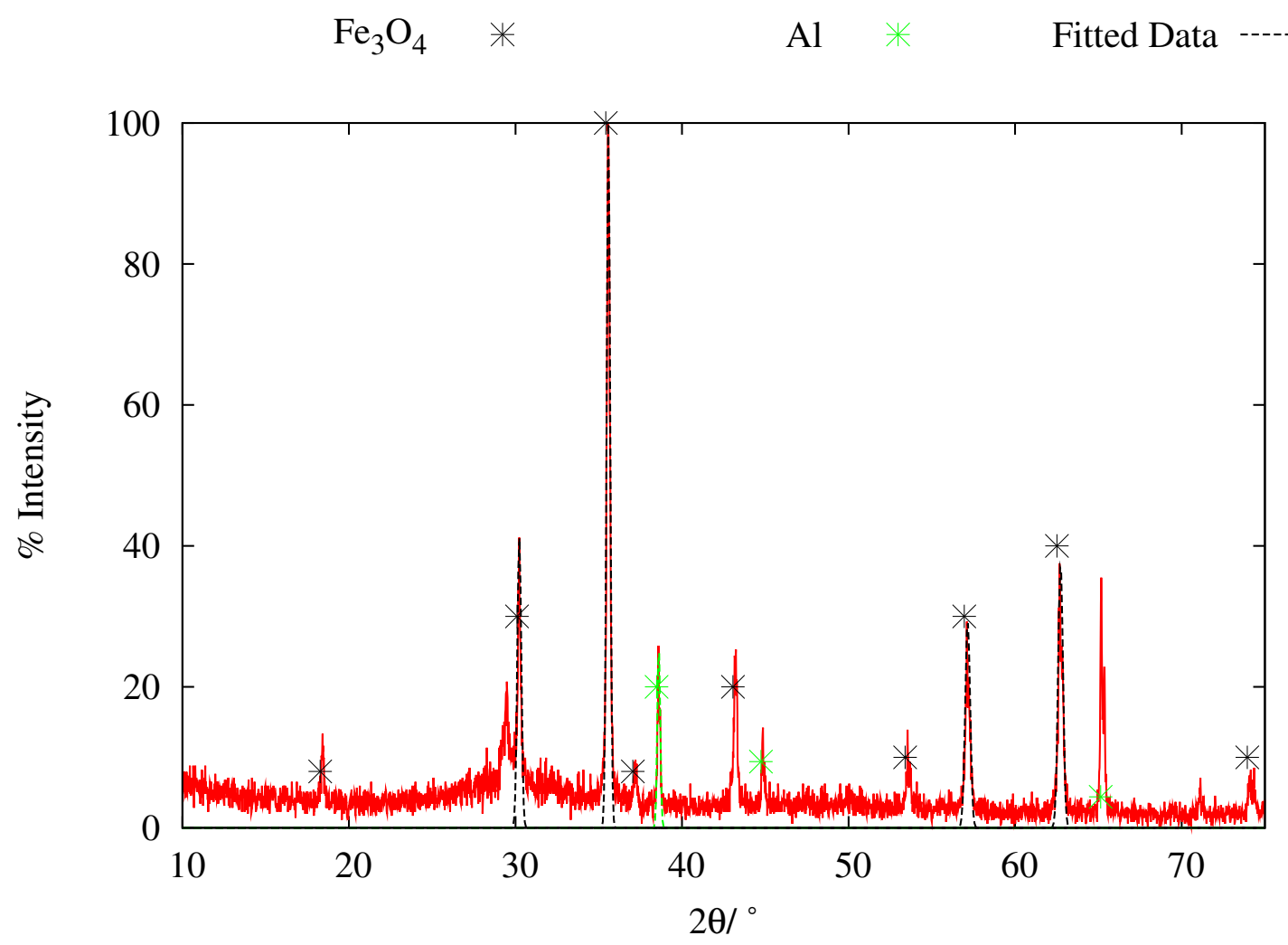

Figure 4.17 X-ray diffraction pattern of $\mathrm{Fe}_{3} \mathrm{O}_{4}$-NCS composite material, prepared with bulk $\mathrm{Fe}_{3} \mathrm{O}_{4}$. 


\subsubsection{Sorption Studies}

The sorption characteristics of the bulk $\mathrm{Fe}_{3} \mathrm{O}_{4}$-NCS composites with respect to phosphate are discussed in detail in Chapter 5. Some studies were made using the $\gamma-\mathrm{Fe}_{2} \mathrm{O}_{3}$-NCS and bulk $\mathrm{Fe}_{3} \mathrm{O}_{4}$-NCS composites for sorption of copper ions.

\subsubsection{Sorption of Copper by Maghemite-Calcium Silicate Composites}

Figure 4.18 shows the sorption characteristics of the $\gamma-\mathrm{Fe}_{2} \mathrm{O}_{3}-\mathrm{NCS}$ composite (containing 10 wt $\left.\% \gamma-\mathrm{Fe}_{2} \mathrm{O}_{3}\right)$ material towards copper. The sorption capacity of the material, as calculated from the data presented in Figure 4.18(a) is $10.8 \pm 0.8 \mathrm{mmol} \mathrm{g}^{-1}$. This compares well to the results presented in Figure 3.5(b), in which a sorption capacity of $10 \pm 1 \mathrm{mmol} \mathrm{g}^{-1}$ was determined. By comparing Figures 4.18(b) and 3.6(b), which depict the sorption of $\mathrm{Cu}^{2+}$ from a $1.6 \mathrm{mmol} \mathrm{dm}{ }^{-3} \mathrm{Cu}\left(\mathrm{NO}_{3}\right)_{2}$ solution by a $\gamma-\mathrm{Fe}_{2} \mathrm{O}_{3}$ NCS and unmodified NCS, it can be seen that the copper is sorbed much faster by the $\gamma-\mathrm{Fe}_{2} \mathrm{O}_{3}$-NCS composite. The sorption is complete after approximately five minutes with the composite, compared to 30 minutes for the unmodified material. This may be due to the smaller particle size (and hence, increased surface area) of the composite materials.

No iron was detected in solution, indicating that the $\gamma-\mathrm{Fe}_{2} \mathrm{O}_{3}$ was stable under the conditions employed. However, it should be noted that the composite material itself was found to dissociate under acidic conditions. 


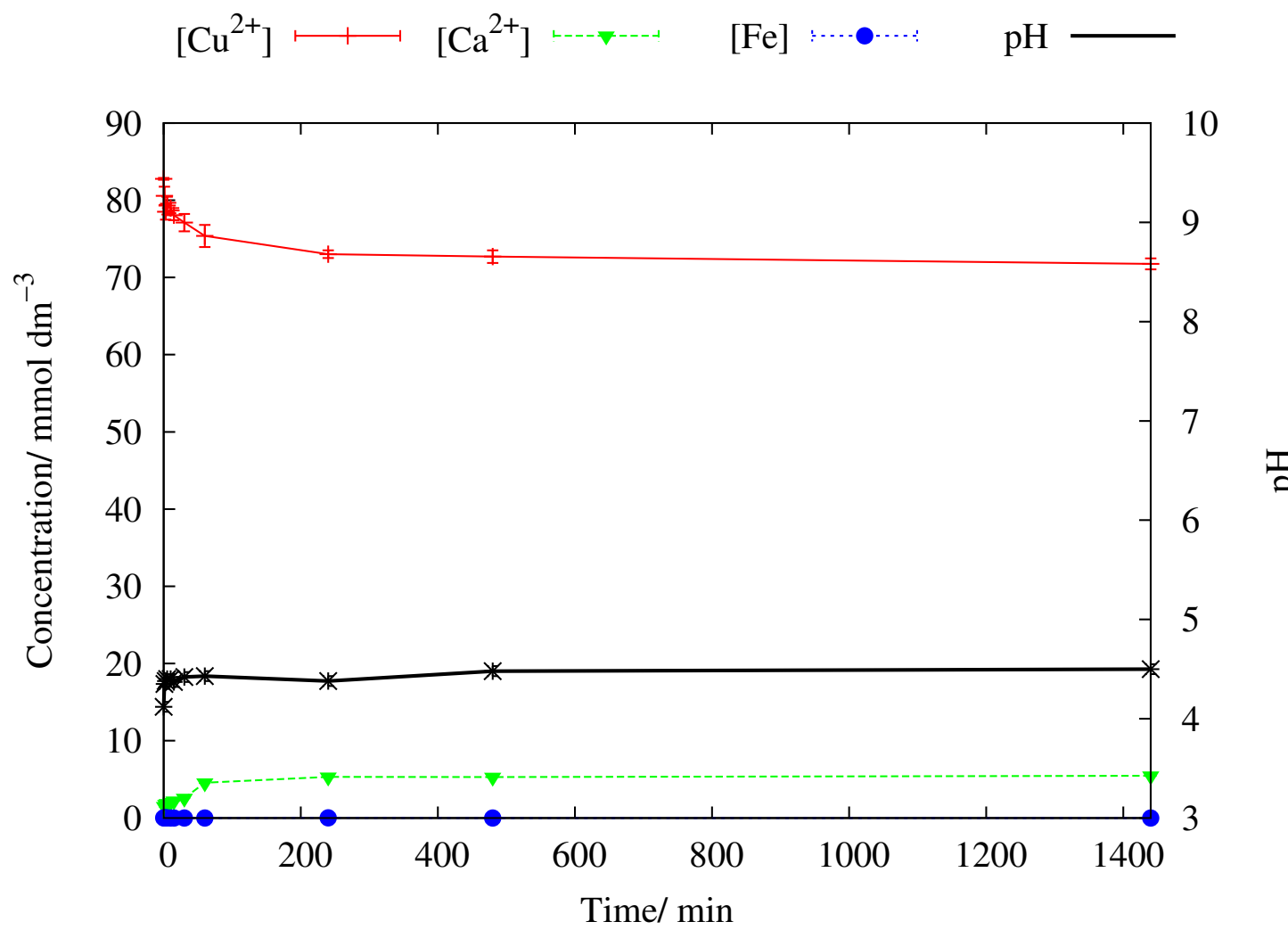

(a)

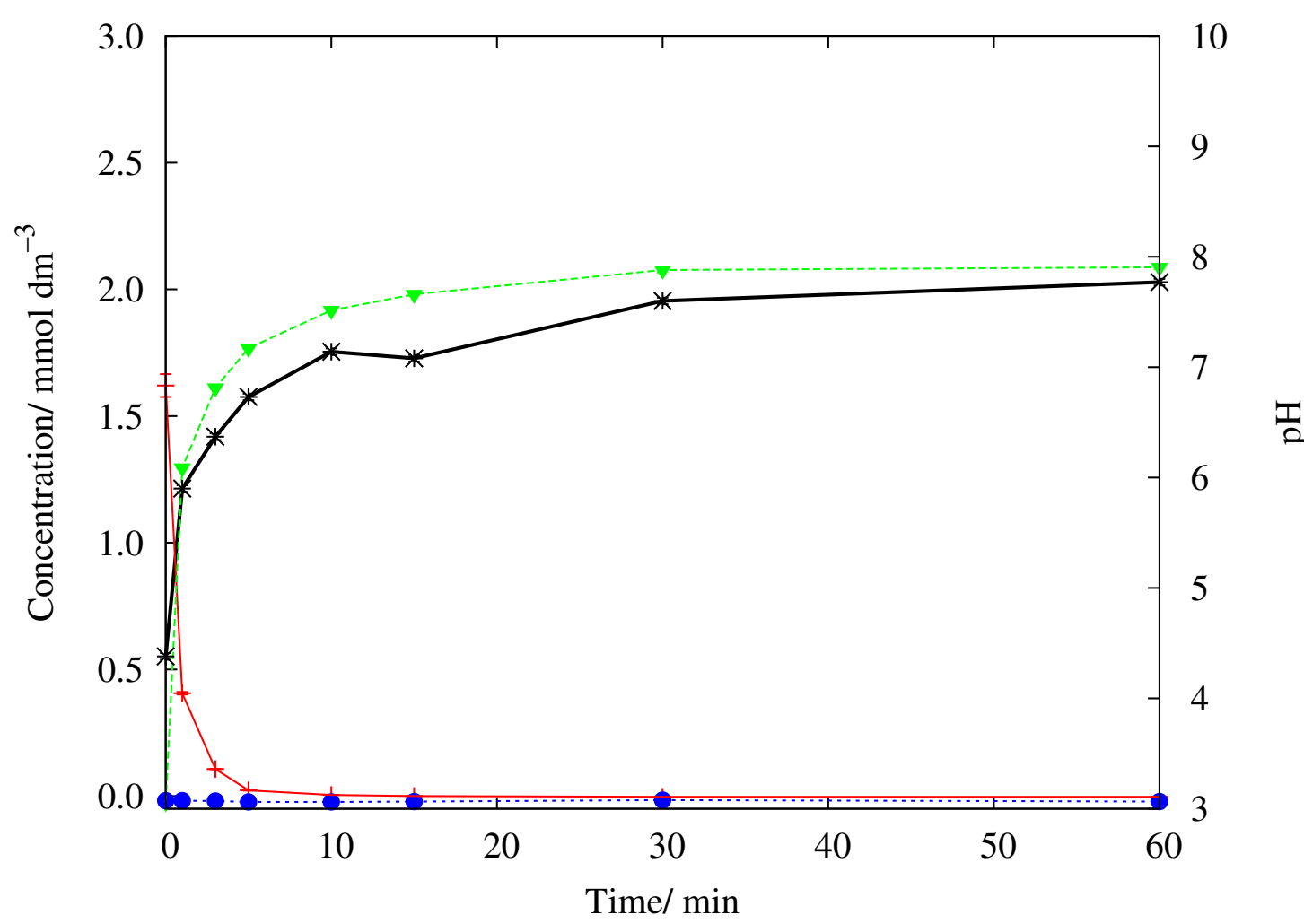

(b)

Figure 4.18 Sorption characteristics of $\gamma-\mathrm{Fe}_{2} \mathrm{O}_{3}-\mathrm{NCS}$ towards $\mathrm{Cu}\left(\mathrm{NO}_{3}\right)_{2}$ at an initial $\mathrm{Cu}^{2+}$ concentration of (a) $80 \mathrm{mmol} \mathrm{dm}^{-3}$, or (b) $1.6 \mathrm{mmol} \mathrm{dm}{ }^{-3}$. Sorbent, containing $10 \mathrm{wt} \% \gamma-\mathrm{Fe}_{2} \mathrm{O}_{3}$, applied at a loading of $1 \mathrm{~g} \mathrm{dm}^{-3}$. Data sets indicate the solution concentration of all species, including any iron leached from the sorbent into solution. 


\subsubsection{Isotherm Measurements of Sorption by Magnetite-Calcium Silicate Com- posite}

Figure 4.19 depicts the sorption isotherm of $\mathrm{Cu}^{2+}\left(\right.$ as $\left.\mathrm{CuCl}_{2}\right)$ by a bulk $\mathrm{Fe}_{3} \mathrm{O}_{4}-\mathrm{NCS}$ composite material containing 25 wt $\% \mathrm{Fe}_{3} \mathrm{O}_{4}$, with the associated parameters for the isotherm models given in Table 4.6. A sorption isotherm of unmodified NCS is provided in the figures for comparison purposes.

Table 4.6 Parameters for copper sorption isotherms as shown in Figure 4.19.

\begin{tabular}{|c|c|c|}
\hline Parameter & & Value \\
\hline \multicolumn{3}{|c|}{ Sorbent } \\
\hline & NCS, Figure 4.19(a) & $\mathrm{Fe}_{3} \mathrm{O}_{4}$-NCS, Figure 4.19(b) \\
\hline \multicolumn{3}{|c|}{ Langmuir Isotherm } \\
\hline$n_{\infty}^{a}$ & 8.75 & 7.05 \\
\hline$K^{b}$ & 221 & 7.14 \\
\hline$R^{2}$ & 0.956 & 0.804 \\
\hline \multicolumn{3}{|c|}{ Freundlich Isotherm } \\
\hline$n_{\infty} a$ & 7.90 & 5.55 \\
\hline$C_{2}$ & 7.93 & 3.70 \\
\hline$R^{2}$ & 0.864 & 0.939 \\
\hline \multicolumn{3}{|c|}{ Tempkin Isotherm } \\
\hline$n_{\infty} C_{1}$ & 0.800 & 0.946 \\
\hline$C_{2}$ & $2.79 \times 10^{3}$ & 503 \\
\hline$R^{2}$ & 0.929 & 0.900 \\
\hline
\end{tabular}

${ }^{a}$ Presented in dimensions of $\mathrm{mmol} \mathrm{g}^{-1}$.

${ }^{b}$ Presented in dimensions of $\mathrm{dm}^{3} \mathrm{mmol}^{-1}$.

It can be seen that that the unmodified NCS filter cake exhibits greatest conformation to the Langmuir isotherm, having an $R^{2}$ value in excess of 0.95 for this model. The calculated maximum sorption capacity for $\mathrm{Cu}^{2+}$ is $8.75 \mathrm{mmol} \mathrm{Cu}^{2+} \mathrm{g}^{-1}$, a value consistent with the maximum sorption sorption capacity of $9 \pm 1 \mathrm{mmol} \mathrm{g}^{-1}$ determined during kinetic sorption studies. By comparison, composite material exhibited a lesser degree of conformity to the Langmuir model, having an $R^{2}$ value of 0.804 , with a maximum sorption capacity of $7.05 \mathrm{mmol} \mathrm{Cu} \mathrm{Cu}^{2+} \mathrm{g}^{-1}$. The lower specific sorption capacity of the composite material (when compared to NCS) is expected, as the composite material contains less NCS on a per mass basis. Thus, the presence of $\mathrm{Fe}_{3} \mathrm{O}_{4}$ does not significantly contribute 
Langmuir

Tempkin

Freundlich

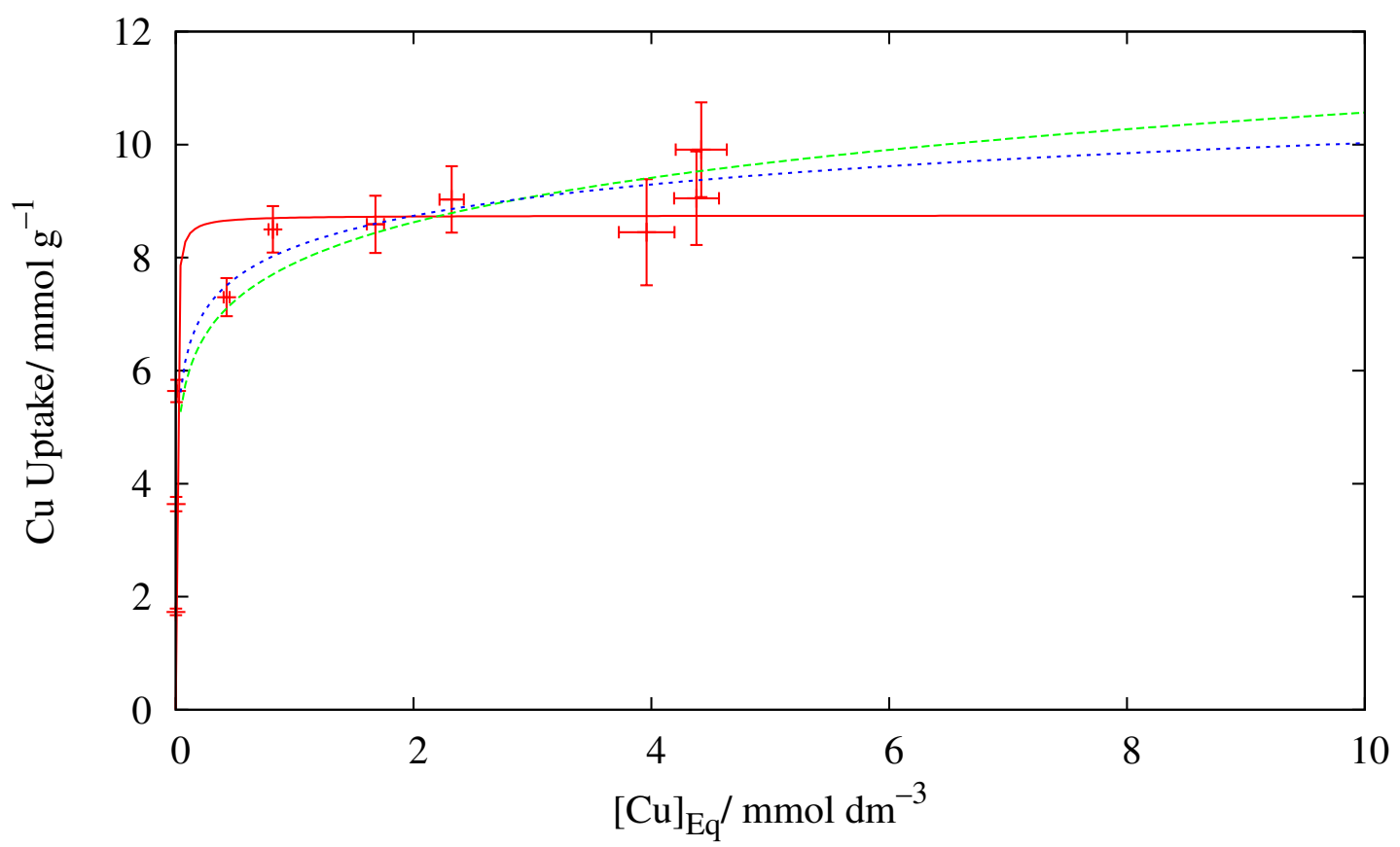

(a)

Langmuir
Freundlich

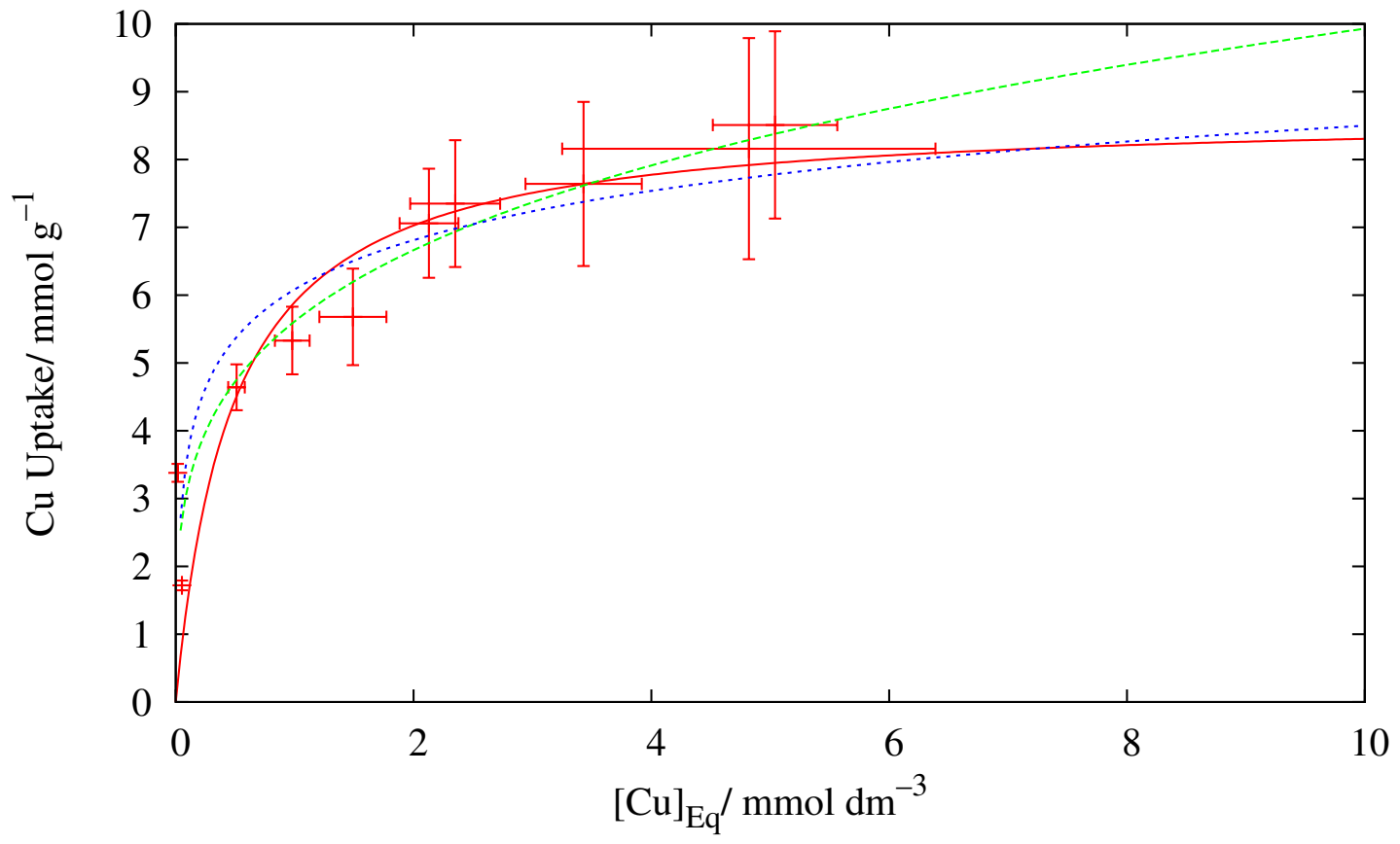

(b)

Figure 4.19 Sorption isotherm for $\mathrm{Fe}_{3} \mathrm{O}_{4}$-NCS composite materials with respect to $\mathrm{Cu}^{2+}$ (as $\mathrm{CuCl}_{2}$ ): (a) sorption by NCS; (b) sorption by $\mathrm{Fe}_{3} \mathrm{O}_{4}$-NCS composite (25 wt \%).

Sorbents applied as filter cake at a loading on $0.5 \mathrm{~g} \mathrm{dm}^{-3}$ (dry basis). 
to the observed sorption capacity of the composite.

The composite material exhibits best conformity to the Freundlich isotherm model (having an $R^{2}$ value of 0.939 ). This lower degree of conformity to the Langmuir isotherm suggests the presence of non-equivalent sorption sites within the composite material. ${ }^{12}$ These are most likely caused to the inclusion of $\mathrm{Fe}_{3} \mathrm{O}_{4}$ within the composite material.

\subsubsection{Electron Microscopy}

A typical electron micrograph of $\gamma-\mathrm{Fe}_{2} \mathrm{O}_{3}$-NCS following sorption of $\mathrm{Cu}^{2+}$ from a $1.6 \mathrm{mmol} \mathrm{dm}{ }^{-3}$ solution of $\mathrm{Cu}\left(\mathrm{NO}_{3}\right)_{2}$ is shown in Figure 4.20. As noted in Chapter 3, sorption of copper from low-concentration solutions has little or no effect on the observed morphology of the NCS particles. However, in this particular example, the initial formation of apparently crystalline copper compounds can be seen.

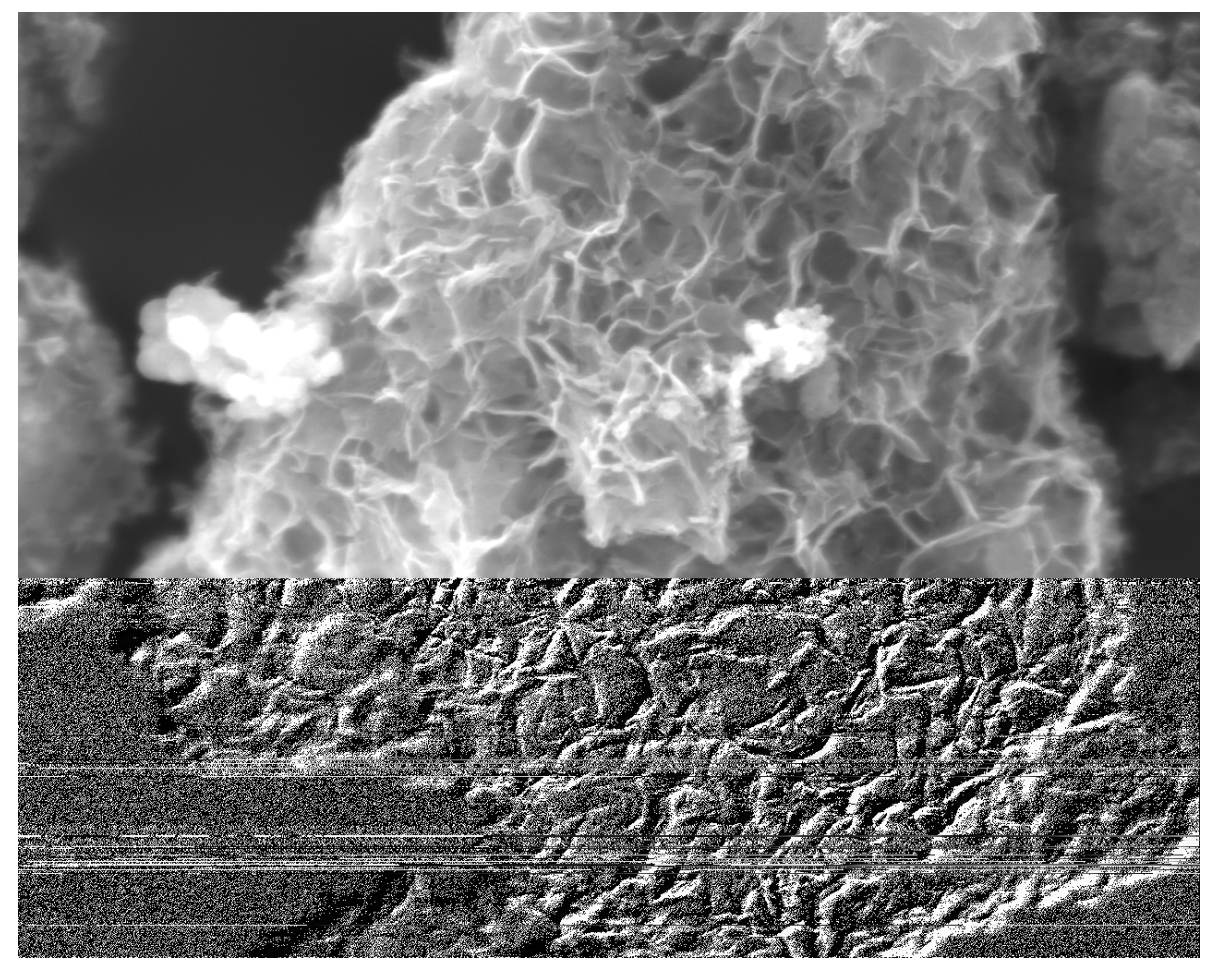

Figure 4.20 Electron micrograph of $\gamma-\mathrm{Fe}_{2} \mathrm{O}_{3}$-NCS following sorption of $\mathrm{Cu}^{2+}$.

Figure 4.21 shows the EDS overlay of the electron micrograph presented in Figure 4.20, a sorption experiment undertaken with $\gamma-\mathrm{Fe}_{2} \mathrm{O}_{3}-\mathrm{NCS}$ and $\mathrm{Cu}\left(\mathrm{NO}_{3}\right)_{2}$ at a concentration of $1.6 \mathrm{mmol} \mathrm{dm}{ }^{-3}$. In the EDS map, silicon has been assigned a red colour, copper is shown 
in green, and iron shown in blue. As can be seen in the EDS overlay, copper is distributed throughout the NCS particle. The intense copper signal observed near the upper left boundary of the NCS shows the initial formation of the $\mathrm{Cu}_{2}\left(\mathrm{NO}_{3}\right)(\mathrm{OH})_{3}$ crystalline phase. The EDS map of iron shows a non-homogeneous distribution throughout the NCS particle. This apparent non-homogeneity is most likely caused by the low contrast of the iron signal. As can be seen in the signal strength indicator to the left of the iron map, only three levels of contrast are present, compared to 6 or more for the other elements. At this level of contrast, the true iron distribution within the sample cannot be fully ascertained.

\subsection{Conclusions}

\subsubsection{Maghemite-Calcium Silicate Composites}

Composite materials containing up to $20 \mathrm{wt} \% \gamma-\mathrm{Fe}_{2} \mathrm{O}_{3}$ were prepared by in-air oxidation of a superparamagnetic $\mathrm{Fe}_{3} \mathrm{O}_{4}$-NCS composite material, which was, in itself prepared from a $\mathrm{Fe}_{3} \mathrm{O}_{4}$ ferrofluid. Particle size analysis of the $\mathrm{Fe}_{3} \mathrm{O}_{4}-\mathrm{NCS}$ composite prior to oxidation of the iron species demonstrated that the presence of $\mathrm{Fe}_{3} \mathrm{O}_{4}$ had a negligible effect on the particle size distribution of the material. Powder X-ray diffraction studies demonstrated the presence of nanosized $\mathrm{Fe}_{3} \mathrm{O}_{4}$ crystallites, with a diameter of approximately $15 \mathrm{~nm}$, which on oxidation resulted in the formation of $\gamma-\mathrm{Fe}_{2} \mathrm{O}_{3}$ particulates of approximately $20 \mathrm{~nm}$ in size. The NCS material retained its desertrose nanoscale morphology in the $\gamma-\mathrm{Fe}_{2} \mathrm{O}_{3}$-NCS composite material, having a slightly decreased particle diameter and slightly reduced specific surface area. The reduction of specific surface area was attributed to the increased mass of the composite rather than a degradation of the NCS structure itself.

The materials were shown to maintain the superparamagnetic character of the initial $\mathrm{Fe}_{3} \mathrm{O}_{4}$. However, the saturation magnetic moment was found to be lower than expected for a pure $\gamma-\mathrm{Fe}_{2} \mathrm{O}_{3}$ phase. This was attributed to the possible formation of an antiferrimagnetic $\alpha-\mathrm{Fe}_{2} \mathrm{O}_{3}$ phase present as an impurity within the samples. No iron could be detected within the composite materials in XPS studies, indicating that the $\gamma$ - 

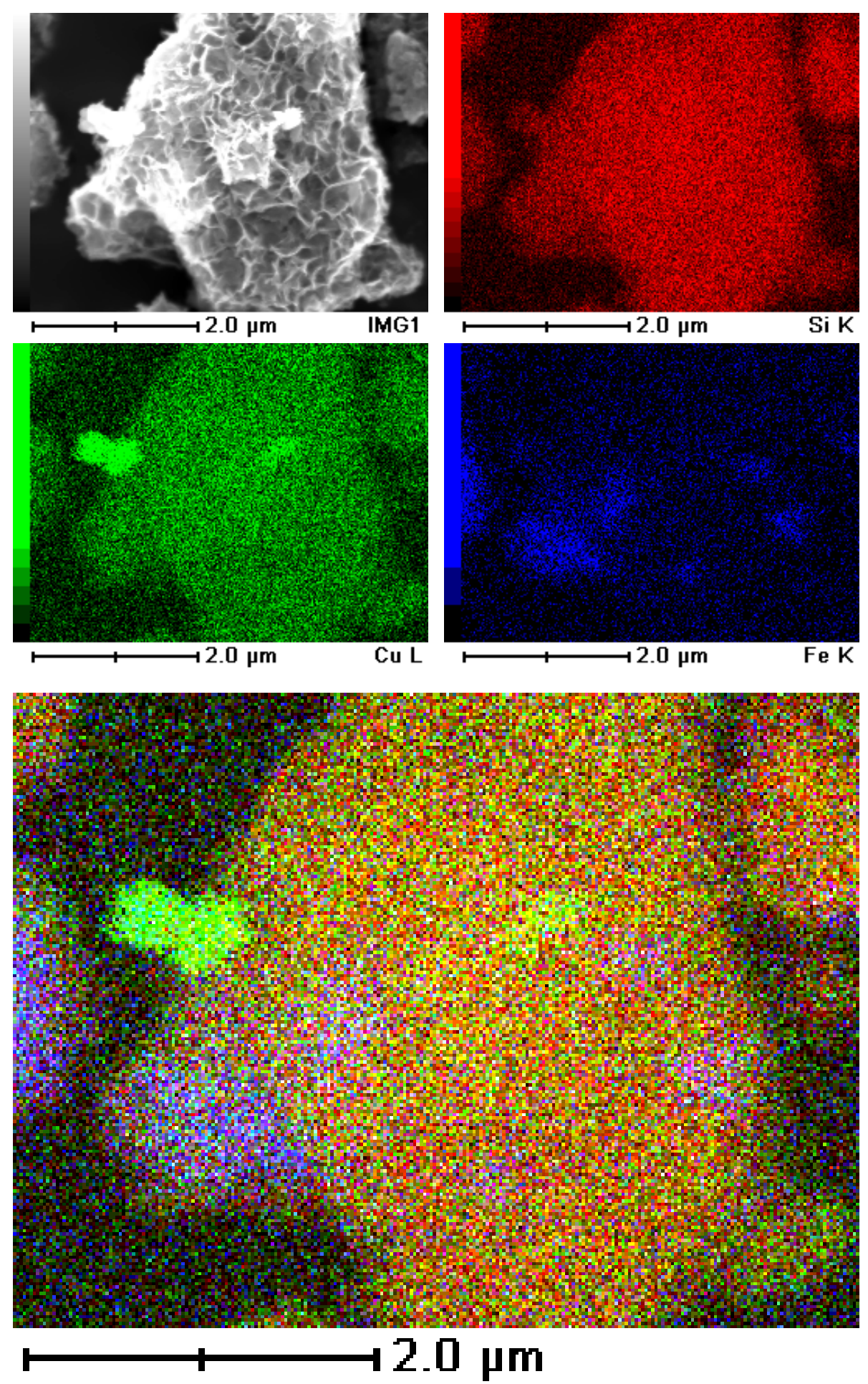

Figure 4.21 EDS overlay of $\gamma$ - $\mathrm{Fe}_{2} \mathrm{O}_{3}$-NCS following sorption of $\mathrm{Cu}^{2+}$ from a $1.6 \mathrm{mmol} \mathrm{dm}{ }^{-3} \mathrm{Cu}\left(\mathrm{NO}_{3}\right)_{2}$ solution: $\mathrm{Si}$ (red); $\mathrm{Cu}$ (green); $\mathrm{Fe}$ (blue). 
$\mathrm{Fe}_{2} \mathrm{O}_{3}$ was incorporated within the NCS structure, and not simply bound to the surface of the material. EDS, with its greater sample penetration depth (micrometre scale compared to picometre scale for XPS) provided evidence for a uniform iron distribution within the composites.

Sorption of copper by derivatives of NCS containing either $\gamma-\mathrm{Fe}_{2} \mathrm{O}_{3}-\mathrm{NCS}$ or $\mathrm{Fe}_{3} \mathrm{O}_{4}-\mathrm{NCS}$ were undertaken. It was found that the presence of maghemite $\left(\gamma-\mathrm{Fe}_{2} \mathrm{O}_{3}-\mathrm{NCS}\right)$ did not degrade the capacity of the NCS. A slight promotion of the sorption rate was noticed, but the maximum sorption capacity remained unchanged $\left(10.8 \pm 0.8 \mathrm{mmol} \mathrm{Cu}^{2+} \mathrm{g}^{-1}\right.$ for a composite containing $10 \mathrm{wt} \% \gamma-\mathrm{Fe}_{2} \mathrm{O}_{3}$ ). However, it was found that the acidic nature of the copper solution caused the composite materials to degrade. This resulted in a partial separation of the NCS and $\gamma-\mathrm{Fe}_{2} \mathrm{O}_{3}$ phases, effectively negating the magnetic characteristics of the material whilst in solution.

\subsubsection{Magnetite-Calcium Silicate Composites}

Composite materials of NCS with bulk magnetite powder were prepared containing up to 25 wt $\% \mathrm{Fe}_{3} \mathrm{O}_{4}$ by dispersion of $\mathrm{Fe}_{3} \mathrm{O}_{4}$ powder in a sodium silicate solution prior to the NCS-forming precipitation reaction with $\mathrm{Ca}(\mathrm{OH})_{2}$. Particle size analysis of the composite material provided no evidence for bimodal size distributions, implying that the $\mathrm{Fe}_{3} \mathrm{O}_{4}$ was fully incorporated within the NCS structure. Unlike with the superparamagnetic composite materials, the bulk magnetite did not oxidise upon heating to $110^{\circ} \mathrm{C}$ in air. No $\gamma-\mathrm{Fe}_{2} \mathrm{O}_{3}$ was formed during the heating process.

The composite materials were found to display a greater degree of conformity with Freundlich isotherm behaviour for the sorption of $\mathrm{Cu}^{2+}$, whereas the NCS material exhibited Langmuir isotherm behaviour under the same conditions. This was attributed to the presence of non-equivalent sorption sites within the composite material, introduced as a result of magnetite inclusion within the material. 


\section{Chapter 5}

\section{Phosphate Sorption by $\mathrm{Fe}_{3} \mathrm{O}_{4}$-NCS Composites}

\subsection{Introduction}

\subsubsection{Phosphorus Speciation}

Inorganic phosphorus, in the aqueous environment, is typically encountered in the form of phosphate, $\mathrm{PO}_{4}^{3-}$, or one of its protonated forms. The equilibria existing between these various forms are shown in Table 5.1. As these reactions are facile, the phosphate system is often employed for the preparation of $\mathrm{pH}$ buffers. In natural waters (typically at a $\mathrm{pH}$ of $7 \pm 1$, the dominant species are $\mathrm{H}_{2} \mathrm{PO}_{4}^{-}$and $\mathrm{HPO}_{4}^{2-}$.

Table 5.1 Equilibria existing between the various protonated forms of phosphate in the aqueous environment (after Alward and Findlay ${ }^{50}$ ).

\begin{tabular}{lr}
\hline Equilibrium & $\mathbf{p} K_{a}$ \\
\hline $\mathrm{H}_{3} \mathrm{PO}_{4} \rightleftharpoons \mathrm{H}^{+}+\mathrm{H}_{2} \mathrm{PO}_{4}^{-}$ & 2.15 \\
$\mathrm{H}_{2} \mathrm{PO}_{4}^{-} \rightleftharpoons \mathrm{H}^{+}+\mathrm{HPO}_{4}^{2-}$ & 7.20 \\
$\mathrm{HPO}_{4}^{2-} \rightleftharpoons \mathrm{H}^{+}+\mathrm{PO}_{4}^{3-}$ & 12.38 \\
\hline
\end{tabular}

\subsubsection{Phosphate in the Environment}

The element phosphorus is relatively abundant, comprising approximately $0.1 \%$ by mass of the Earth's crust. ${ }^{3}$ It is never found as the pure element in Nature, and almost always exists as phosphate, $\mathrm{PO}_{4}^{3-}$ or one of its protonated or polymetric derivatives. The labile nature of the phosphate protonation process often leads to its use as a buffering agent to control $\mathrm{pH}$. Phosphorus is an element vital to all known forms of life; DNA is an organophosphate polymer ${ }^{119}$ whereas the ADP/ATP energy cycle depends on the cleavage of phosphate from ATP (or addition of phosphate to ADP). ${ }^{120}$

Due to the low solubility of most phosphate minerals, especially iron, calcium and 
aluminium phosphates (Table 5.2), biologically available phosphorus is often present in very low concentrations in the natural environment. Due to the biological dependence on phosphate, the rate of biological activity, such as algal growth, is often limited by this nutrient. As such, should waste streams (normally from anthropogenic sources, such as municipal effluent or agricultural run-off) with significant concentration of bio-available phosphorus be introduced into the environment, pre-existing life thrives. This can, for example, lead to eutrophication of lakes or other water bodies; sudden growth of algae and its subsequent bacterial decomposition leads to a depletion of dissolved oxygen. This drastically affects the balance of the local ecosystem, often eliminating higher order animals, such as fish, from the area. ${ }^{121}$

Table 5.2 Common metal species encountered as counter-ions in phosphate precipitates, after Sharpley. ${ }^{122}$

\begin{tabular}{ll}
\hline Metal Ion & Common Precipitates \\
\hline $\mathrm{Ca}^{2+}$ & $\mathrm{Ca}_{3}\left(\mathrm{PO}_{4}\right)_{2}$ \\
& $\mathrm{Ca}_{5}(\mathrm{OH})\left(\mathrm{PO}_{4}\right)_{3}$ \\
$\mathrm{Fe}^{2+}, \mathrm{Fe}^{3+}$ & $\mathrm{Fe}_{3}\left(\mathrm{PO}_{4}\right)_{2}$ \\
& $\mathrm{Fe}_{\mathrm{x}}(\mathrm{OH})_{\mathrm{x}}\left(\mathrm{PO}_{4}\right)_{\mathrm{z}}$ \\
$\mathrm{Al}^{3+}$ & $\mathrm{Al}_{\mathrm{x}}(\mathrm{OH})_{\mathrm{y}}\left(\mathrm{PO}_{4}\right)_{\mathrm{z}}$ \\
\hline
\end{tabular}

The low bioavailability of phosphate in the environment has created a great demand worldwide for agricultural fertilisers containing this vital nutrient. This has lead, over the past hundred years or so, to the depletion of much of the world's readily accessible reserves of phosphate minerals. In the Pacific region, one of the prime examples of this is the island of Nauru. Phosphate mining was commenced on Nauru in 1906; all reserves of phosphate ore were exhausted by the mid 1990s. ${ }^{123}$ It has been estimated that there are perhaps 80-100 years worth of economically recoverable phosphate reserves worldwide. ${ }^{3}$ After these reserves have been exhausted, lower grade ores, often contaminated with toxic metal ions, such as cadmium, must be used. The alternative to this is a phosphate recycling system, whereby phosphate is recovered from a waste stream at, or near, its source, preventing environmental degradation. The thus recovered phosphate may then be reused, as, for example a feedstock for the fertiliser industry. ${ }^{14}$ 


\subsubsection{Phosphate Sorption}

Materials used for phosphate sorption are often based on aluminium-, ${ }^{124-128}$ calcium-, ${ }^{33-35,129-131}$ or iron-containing ${ }^{127,132-134}$ materials due to the low solubility of the poshpate minerals of these cations (Table 5.3). In recent years, much work has been focused on sorbing phosphate via precipitation as calcium phosphate phases, due to their low solubility product and the low cost of calcium-containing sorbent precursors. Of the various calcium phosphates, the apatites have the lowest solubility; $\mathrm{p} K_{s}$ for hydroxyapatite, $\mathrm{Ca}_{5}\left(\mathrm{PO}_{4}\right)_{3}(\mathrm{OH})$, ranges from 54.4 to 58.5 at $25^{\circ} \mathrm{C}$. ${ }^{135}$ Despite the low solubility of the apatites, calcium and phosphate are readily found in solution at concentrations far exceeding that for the precipitation of apatite phases, due to the large activation energy of the precipitation step. It has previously been shown that the initial calcium phosphate precipitate consists mainly of $\mathrm{Ca}^{2+}$ and $\mathrm{H}_{2} \mathrm{PO}_{4}^{-}$ions. ${ }^{136}$ The phosphate ion, $\mathrm{PO}_{4}^{3-}$ is not encountered in this initial precipitate, which subsequently ages to form the apatite phase over a period of several hours (Equation (5.1)). The activation energy for this ageing step process been reported as between $95-105 \mathrm{~kJ} \mathrm{~mol}^{-1} \cdot 136,137$

Table 5.3 Calcium compounds of phosphorus commonly encountered in soils as reported by Brady. ${ }^{138}$

\begin{tabular}{llr}
\hline Compound & Formula $^{a}$ & Solubility Product, $\mathbf{p} K_{s}{ }^{b}$ \\
\hline Fluoroapatite & $\mathrm{Ca}_{5}\left(\mathrm{PO}_{4}\right)_{3} \mathrm{~F}$ & $58.1^{139}$ \\
Hydroxyapatite & $\mathrm{Ca}_{5}\left(\mathrm{PO}_{4}\right)_{3} \mathrm{OH}$ & $56.0^{139}$ \\
Tricalcium phosphate & $\mathrm{Ca}_{3}\left(\mathrm{PO}_{4}\right)_{2}$ & $28.9^{50}$ \\
Monetite & $\mathrm{CaHPO}_{4}$ & $6.60^{139}$ \\
Brushite & $\mathrm{CaHPO}_{4} \cdot 2 \mathrm{H}_{2} \mathrm{O}$ & $6.60^{139}$ \\
\hline
\end{tabular}

${ }^{a}$ The formula has been adjusted to coincide with the reported solubility product.

${ }^{b}$ These references are for the solubility products, not listed by Brady.

$$
x \mathrm{H}_{2} \mathrm{PO}_{4}^{-}+y \mathrm{Ca}^{2+} \longrightarrow \mathrm{Ca}\left(\mathrm{H}_{2} \mathrm{PO}_{4}\right)_{2} \longrightarrow \mathrm{Ca}\left(\mathrm{HPO}_{4}\right) \cdot 2 \mathrm{H}_{2} \mathrm{O} \longrightarrow \longrightarrow \mathrm{Ca}_{5}\left(\mathrm{PO}_{4}\right)_{3} \mathrm{OH}
$$




\subsubsection{Biological Removal}

Due to its prominent biological role and inherently low solubility, phosphate is often a limiting nutrient in ecological systems. For this reason, biological systems (such as activated sludge) are widely implemented for the purpose of removing phosphate from wastewater streams, particularly municipal wastewater. ${ }^{140-142}$ Bacteria commonly reported as being used for phosphate removal include Microthrix parvicella, Acinetobacter spp., Microlunatus phosphovorus and Lampropedia spp. ${ }^{140}$ Under appropriate operating conditions, an optimised biological phosphate removal system can remove over $80 \%$ of the phosphate present in municipal wastewater, typically resulting in an effluent having a concentration less than $30 \mu \mathrm{mol} \mathrm{P} \mathrm{dm}{ }^{-3}$. ${ }^{141}$ Such systems typically operate in a three stage process:

- The influent is initially permitted to react with the microbiological sludge in an anaerobic environment. In this step, the bacteria metabolise organic components of the wastewater, typically volatile fatty acids (which are converted into polyhydroxyalkanoates). ${ }^{142}$ This stage results in an increase in the phosphate concentration of the wastewater; the bacteria degrade their reserves of polyphosphates (releasing phosphate) to provide energy for the metabolises of the fatty acids.

- In the second step, the system is aerated. The introduction of oxygen to the system triggers a growth phase in the bacteria. The stored fatty acids are used to regenerate the reserves of polyphosphates, a process which typically results in extra phosphate removal from solution, so-called "luxury uptake" (phosphate uptake in excess of that released in the anaerobic step). The growth phase also results in removal of moderate quantities of nitrogen nutrients $\left(\mathrm{NH}_{3}\right.$ and $\left.\mathrm{NO}_{3}^{-}\right)$from solution.

- The final step is a settling phase. The sludge is separated from the effluent and returned to the anaerobic stage. Excess sludge may be processed to recover the phosphates or otherwise discarded.

You and Ouyang ${ }^{143}$ have reported a modification to this generic process whereby in addition to the phosphate-removing organisms in the sludge, bacteria optimised for 
denitrification are introduced to the aerobic stage. They report $>99 \%$ phosphorus removal and $82.9 \%$ nitrogen removal from a synthetic wastewater influent containing $0.15 \mathrm{mmol} \mathrm{P} \mathrm{dm}^{-3}$ and $2.76 \mathrm{mmol} \mathrm{N} \mathrm{dm}^{-3}$, predominantly present as phosphate and ammonia respectively.

Being a biological process, such systems have the advantage of being inexpensive to operate as they typically require no inputs of chemicals to achieve phosphate removal. Such systems also have disadvantages:

- The system requires a supply of fatty acids to effectively remove phosphate. It has been suggested that fatty acids should be present in a seven to nine times excess (mass basis) over phosphorus ${ }^{141}$ to achieve optimal phosphate removal. This is typically encountered at a $\mathrm{BOD}_{5}: \mathrm{P}$ ratio in excess of $20: 1 .^{\dagger}$ While this ratio is usually exceeded for municipal sewage, this is not always the case for phosphatecontaining wastewaters from other sources such as agricultural run-off or various industrial processes. In these cases biological treatment may not be possible.

- The system is very susceptible to changes in the temperature, composition and flow-rate of the influent. It has been suggested that many biological treatment systems may take as much as four weeks to re-achieve optimal phosphate removal following radical disturbances in the temperature, flow-rate or composition of the influent. ${ }^{141}$ Changes in the composition of other biologically significant chemical species, such as $\mathrm{Na}^{+}, \mathrm{K}^{+}$and $\mathrm{CH}_{3} \mathrm{COO}^{-}$can significantly alter the performance of the system. Acetate, $\mathrm{CH}_{3} \mathrm{COO}^{-}$, in particular will alter the performance of the system as bacteria will metabolise this species in preference to fatty acids. The presence of toxic components in the influent, such as some of the metal ions listed in Table 1.2, is highly detrimental to the operation of the system.

- The system can suffer biological contamination. If a fibrous bacterial species, such as Nostocoida limicola becomes established in the sludge system, the sedimentation and clarification treatments applied to the effluent become less efficient. This means

${ }^{\dagger} \mathrm{BOD}_{5}$ is the Biological Oxygen Demand over 5 days and is indicative of the mobilisable organic content of the sample. ${ }^{10}$. The 20:1 ratio is reported on a mass per mass basis. 
that the overall phosphate removal of the system decreases, as sludge material with a high phosphate concentration may be discharged in the effluent stream. ${ }^{141}$

\subsubsection{Chemical Removal}

Due to the low solubility of many of the phosphate minerals, its removal from wastewater often relies on the addition of a chemical to precipitate a phosphate salt. While such a method is simple, it often results in large volumes of sludge, the disposal of which can be problematic. Common precipitants used in treatment processes include the mineral alum $\left(\mathrm{KAl}\left(\mathrm{SO}_{4}\right)_{2} \cdot 12 \mathrm{H}_{2} \mathrm{O}\right)$ or $\mathrm{Al}(\mathrm{OH})_{3},{ }^{125,126} \mathrm{Fe}^{2+} / \mathrm{Fe}^{3+}$ salts or hydroxides, ${ }^{134,144}$ $\mathrm{MgO},{ }^{145,146}$ or calcite. ${ }^{130}$ The use of magnesium to precipitate phosphate minerals is often desirable in municipal (or other ammonical) wastewater treatment as the precipitate formed is struvite, $\mathrm{NH}_{4} \mathrm{MgPO}_{4} \cdot 6 \mathrm{H}_{2} \mathrm{O}$, meaning that ammonium is removed concurrently. In many cases, struvite may be recovered and used as a fertiliser. However, use of the precipitation technique often results in large quantities of sludge, the disposal of which can be problematic. Because to this, sorbent materials, containing the precipitant metal ions, are often used instead. Sorbent materials usually exhibit more desirable processibility characteristics, when compared to precipitants, an important consideration when sorption is undertaken on a large scale.

\subsubsection{Aluminium and Iron}

Sorbent materials derived from soils have been studied for phosphate sorption. This topic is often approached both from the direction of phosphate sorption ${ }^{147,148}$ and phosphate release ${ }^{149,150}$ (due to the importance of this nutrient for agriculture). Sorption of phosphate by soils typically occurs through sorption of the ion by iron and aluminium minerals in the soil matrix. Phosphate sorption by aluminium based materials is believed to proceed via the displacement or exchange of hydroxyl or water ligands on the sorbent surface for phosphate ions:

$$
\mathrm{Al}(\mathrm{OH})_{x}+\mathrm{H}_{2} \mathrm{PO}_{4}^{-} \rightleftharpoons \mathrm{Al}(\mathrm{OH})_{(x-1)}\left(\mathrm{H}_{2} \mathrm{PO}_{4}\right)+\mathrm{OH}^{-}
$$


This process is energetically favourable at slightly acidic to neutral $\mathrm{pH}$ values of 4 $7^{124}$ and will occur in many aluminium-containing minerals such as zeolites, ${ }^{128}$ fly ash, ${ }^{129}$ clays, ${ }^{151,152}$ and micas. ${ }^{124}$ A similar process has been reported to occur for ironbased sorbent materials such as iron hydroxides, ${ }^{153-155}$ blast furnace slag, ${ }^{156}$ or synthetic sorbents containing combined aluminium and iron oxides. ${ }^{157}$ The sorption behaviour of such materials can often be modelled by the Freundlich isotherm, ${ }^{128,154,155}$ although the conformation towards the Langmuir isotherm has also been observed. ${ }^{156}$ Many materials otherwise inert with respect to phosphate sorption have been activated by pretreatment with aluminium or iron ions, such as sand, ${ }^{127,158}$ polymer fibres, ${ }^{159}$ or biological waste products such as orange peel. ${ }^{128}$

\subsubsection{Calcium}

When one considers the sorption of phosphate by calcium based materials, it is prudent to examine $\mathrm{CaO}-\mathrm{SiO}_{2}-\mathrm{P}_{2} \mathrm{O}_{5}$ glasses, such as Bioglass ${ }^{\circledR}$, used as biological implants. These materials are often implanted in patients with severe injuries to, or deformities of the skeletal structure. After implantation, apatite forms in the interface between the implant and the original bone. The strength of this interfacial apatite often exceeds that of the original bone. Studies of the apatite formation have suggested the following mechanism, as presented in a review of the subject given by Hench: ${ }^{160}$

- Ion exchange of alkali cations for $\mathrm{H}^{+}$, increasing the $\mathrm{pH}$ near the surface region of the glass.

- Condensation of dissolved silica to form a permeable surface layer.

- Diffusion of calcium and phosphate ions through this layer.

- Precipitation of an amorphous calcium phosphate phase.

- Ageing of this precipitate, resulting in formation of the more thermodynamically stable apatite phase. 
All of these steps, with the possible exception of the condensation of the dissolved silica are probably relevant to the sorption of phosphate by calcium-based systems. One can suggest the following:

- Ion exchange of surface $\mathrm{Ca}^{2+}$ ions for $\mathrm{H}^{+}$, having the dual effect of raising the $\mathrm{pH}$ and increasing the $\mathrm{Ca}^{2+}$ concentration in the local environment.

- Diffusion of phosphate from the bulk of the solution to this surface layer.

- Precipitation of an amorphous calcium phosphate phase on the calcium silicate.

- Ageing of this phosphate to form the apatite phase.

It has previously been noted that many wastewaters contain calcium and phosphate at supersaturated concentrations with respect to the precipitation of $\mathrm{Ca}_{5}\left(\mathrm{PO}_{4}\right)_{3} \mathrm{OH}$ (Section 5.1.3). Due to this supersaturation, calcium seed materials are often used to initiate apatite precipitation. Typically calcite, $\mathrm{CaCO}_{3}$, is used to achieve this, ${ }^{130,161}$ although apatite ${ }^{162}$ or dolomite $\left(\mathrm{CaMg}\left(\mathrm{CO}_{3}\right)_{2}\right)^{131}$ are also used. In many cases, the calcium phosphate phases precipitated in this way can be used as feedstocks for the phosphate fertiliser industry. Berg et al., ${ }^{161}$ for example, demonstrated calcite induced precipitation of apatite from municipal wastewater. The sorbed product contained 25 wt $\% \mathrm{P}_{2} \mathrm{O}_{5}$, higher than the $23 \mathrm{wt} \%$ required for phosphate fertiliser feedstock.

The use of portland cement as a sorbent material for phosphate has also been reported. ${ }^{14}$ A phosphate sorption capacity of $0.9 \mathrm{mmol} \mathrm{P} \mathrm{g}^{-1}$ was reported, with the sorption process exhibiting characteristics consistent with the Frumkin isotherm.

\subsubsection{Phosphate Sorption by NCS}

Southam, in $2004^{33}$ and $2005^{34}$ conducted extensive studies of the characteristics of unmodified NCS with regard to its sorption of phosphate. It was found that the material possessed a maximum sorption capacity for phosphate of $1.94 \mathrm{mmol} \mathrm{P} \mathrm{g} \mathrm{g}^{-1} \mathrm{NCS}$. Maximum phosphate sorption was achieved when the phosphate was initially present as 
the $\mathrm{H}_{2} \mathrm{PO}_{4}^{-}$ion. When $\mathrm{HPO}_{4}^{2-}$ or $\mathrm{PO}_{4}^{3-}$ were used as the phosphate source, a maximum sorption capacity of $1.78 \mathrm{mmol} \mathrm{P} \mathrm{g}{ }^{-1} \mathrm{NCS}\left(\mathrm{HPO}_{4}^{2-}\right)$ or $1.56 \mathrm{mmol} \mathrm{P} \mathrm{g}{ }^{-1} \mathrm{NCS}\left(\mathrm{PO}_{4}^{3-}\right)$ respectively, was attained. The 2-ethoxyethanol washed form of NCS was found to be a better sorbent material than the water washed or $\mathrm{pH}$ adjusted forms. This was attributed to the 2-ethoxyethanol washed material having a higher surface area than the water washed material, and a higher calcium content than the $\mathrm{pH}$ adjusted NCS material.

The sorption of phosphate by NCS was found to occur via a two-step mechanism, both parts of which exhibited second order kinetics. In the first step, the NCS forms what was presumed to be a positively charged intermediate, either by reaction with $\mathrm{H}_{2} \mathrm{PO}_{4}^{-}$or $\mathrm{H}^{+} .^{\dagger}$ In the second step of the sorption process, the intermediate reacted with the phosphate, removing it from solution. The first step (reaction of NCS with $\mathrm{H}^{+}$) was found to be rate limiting for the sorption reaction. Thus, phosphate sorption from solutions of $\mathrm{H}_{2} \mathrm{PO}_{4}^{-}$ occurred faster than sorption from $\mathrm{HPO}_{4}^{2-}$ or $\mathrm{PO}_{4}^{3-}$ solutions due to the lower initial $\mathrm{pH}$ of the $\mathrm{H}_{2} \mathrm{PO}_{4}^{-}$solution. Sorption isotherms, when measured at high loadings of NCS (up to $25 \mathrm{~g} \mathrm{NCS} \mathrm{dm}^{-3}$ ) indicated that the NCS exhibited a better conformity to the Freundlich isotherm than the Langmuir or Tempkin isotherms.

It was found by Southam that the brushite phase, $\mathrm{CaHPO}_{4} \cdot 2 \mathrm{H}_{2} \mathrm{O}$, is formed during the sorption of $\mathrm{H}_{2} \mathrm{PO}_{4}^{-}$by NCS, rather than the more thermdynamically stable apatite phase, $\left(\mathrm{Ca}_{5}\left(\mathrm{PO}_{4}\right)_{3}(\mathrm{OH})\right)$. It is known that brushite is an intermediate during the initial stages of hydroxyapatite formation. ${ }^{136}$ Southam suggested that that the presence of an initial brushite precipitate, or the NCS surface itself, retarded formation of the apatite phase.

It has also been shown by Lai ${ }^{163}$ that the reinforced NCS material exhibits similar adsorption characteristics to those observed by Southam. As the reinforced material has a lower calcium content than the 2-ethoxyethanol washed material, the sorption capacity is lower, and the material takes longer to attain equilibrium. The reported sorption capacity of the reinforced NCS was $91 \%$ that of the 2-ethoxyethanol washed material.

\footnotetext{
${ }^{\dagger}$ This probably occurs at a $\mathrm{Si}-\mathrm{O}-\mathrm{Ca}---$ site on the NCS surface, forming $\mathrm{Si}-(\mathrm{OH})^{+}-\mathrm{Ca}---$, thereby reducing the strength of the $\mathrm{Ca}-\mathrm{O}$ bond and making the calcium more labile.
} 


\subsection{Results}

Experiments were undertaken to evaluate the sorption characteristics of $\mathrm{Fe}_{3} \mathrm{O}_{4}$-NCS composite materials with respect to unmodified NCS. The magnetic properties of the NCS composite were employed by using the composite material as the sorbent in a continuous uptake process.

\subsubsection{Isotherm Studies}

The sorption of phosphate was investigated in respect to the magnetite content of the silicate (Figure 5.1). As expected, the sorption capacity of the composite decreased as the magnetite content increased. When related to the actual mass of silicate present (as opposed to the total mass of the composite), the sorption capacity was constant within the limits of uncertainty. The maximum sorption was found to be $5.1 \pm 0.5 \mathrm{mmol} \mathrm{\textrm {P } \mathrm { g } ^ { - 1 }}$ silicate. By comparison, the sorption of phosphate for a sample of $100 \%$ magnetite was found to be $0.4 \pm 0.2 \mathrm{mmol} \mathrm{P} \mathrm{g}^{-1}$. Therefore, the $\mathrm{Fe}_{3} \mathrm{O}_{4}$ only alters the specific sorption capacity of the composite material insofar as it reduces the quantity of NCS in a given mass of the composite material.

Elemental analysis has previously shown that the calcium silicate (once dried at $110^{\circ} \mathrm{C}$ ) contains approximately $7.1 \mathrm{mmol} \mathrm{Ca} \mathrm{g}^{-1}(28 \mathrm{wt} \% \mathrm{Ca})$. Previous studies ${ }^{34}$ have also shown by XRD analysis that the brushite, $\mathrm{CaHPO}_{4} \cdot 2 \mathrm{H}_{2} \mathrm{O}$, is the preferred crystalline phase formed upon phosphate sorption by NCS. The Ca:P ratio of 1.4:1 in the sorbed product is greater than that found in brushite, $(1: 1)$, and is also inconsistent with that found in apatite $\left(\mathrm{Ca}_{5}\left(\mathrm{PO}_{4}\right)_{3}(\mathrm{OH})\right)$, which has a $\mathrm{Ca}: \mathrm{P}$ ratio of 1.67:1. In the isotherm measurements shown later (Figure 5.2(a)), that at the NCS loading of $0.5 \mathrm{~g} \mathrm{dm}^{-3}$, saturation of NCS with phosphate has been achieved - no more phosphate is capable of being sorbed. Because of this, if brushite were being formed, some $\mathrm{Ca}^{2+}$ would remain uncoordinated to phosphate. It is possible that a mixture of apatite and brushite was formed, which would account for the observed $\mathrm{Ca}: \mathrm{P}$ ratio.

Attempts to determine the amount of phosphate present in the washed and dried samples 
after sorption via digestion in nitric acid were inconsistent. The results from the digestion process indicated the presence of much more phosphate than could be present in the sample. This was attributed to the digestion process interfering with the results, probably due to the acid digestion process forming reactive silicate oligomers.

The presence of silicate ions $\left(\mathrm{H}_{x} \mathrm{SiO}_{4}^{(4-x)-}\right)$ are known to produce positive interference in the determination of phosphate by the molybdenum blue photometric technique. ${ }^{10}$ To solve this issue when isotherm sorption measurements were conducted, a correction for the silica interference was made by equilibrating a sample of NCS in MilliQ water containing no phosphate. The silicate introduced a positive interference in the phosphate concentration on the order of $0.002 \mathrm{mmol} \mathrm{dm}^{-3}$ under normal conditions. Under digestion conditions, this interference was significantly higher (by orders of magnitude) and useful data could not be obtained due to this.

Isotherm studies were conducted with a calcium silicate composite containing 25 wt $\%$ magnetite (dry basis). Initially, the NCS composite was applied at a loading of $0.5 \mathrm{~g} \mathrm{dm}^{-3}$ (dry basis). A Levenberg-Marquardt algorithm was used for the purpose of performing

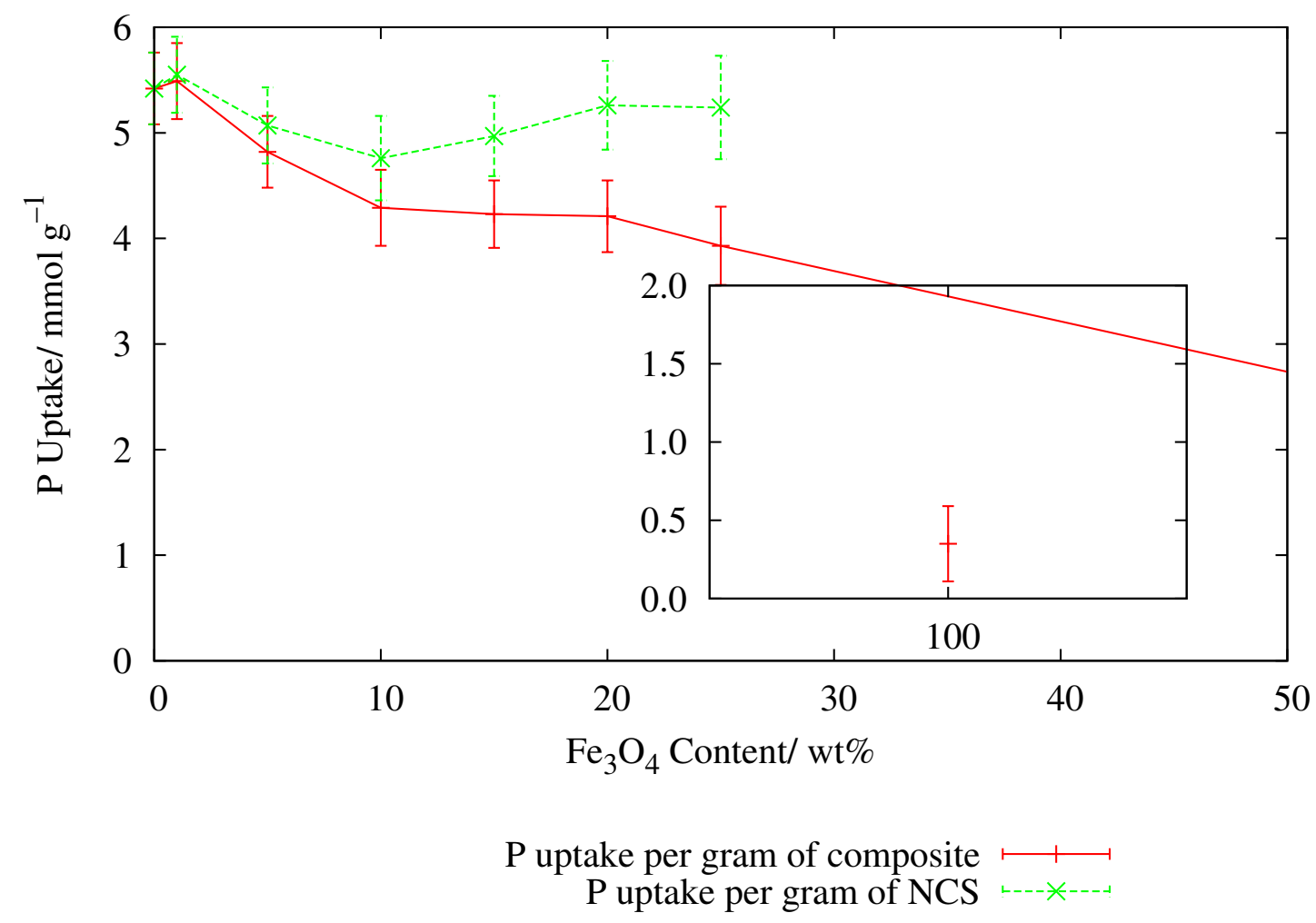

Figure 5.1 Phosphate sorption capacity of $\mathrm{Fe}_{3} \mathrm{O}_{4}-\mathrm{NCS}$ composite materials at a composite loading of $0.5 \mathrm{~g} \mathrm{dm}^{-3}$. 
a non-linear least-squares fit of the isotherm models to the experimental data set. The data were not initially transformed to conform to a linear interpretation of the isotherm models, as discussed in Chapter 1.

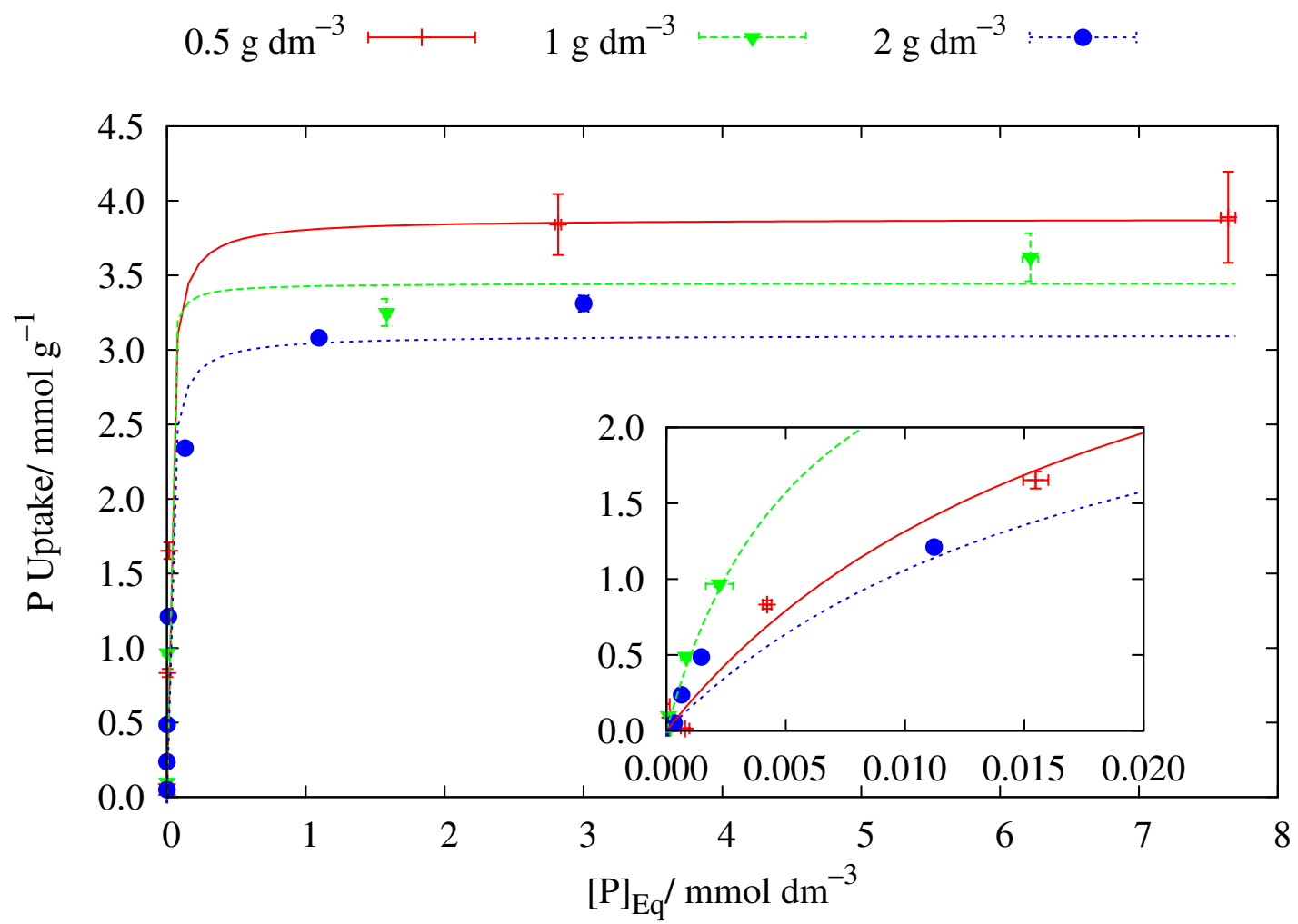

(a) Langmuir isotherm model.

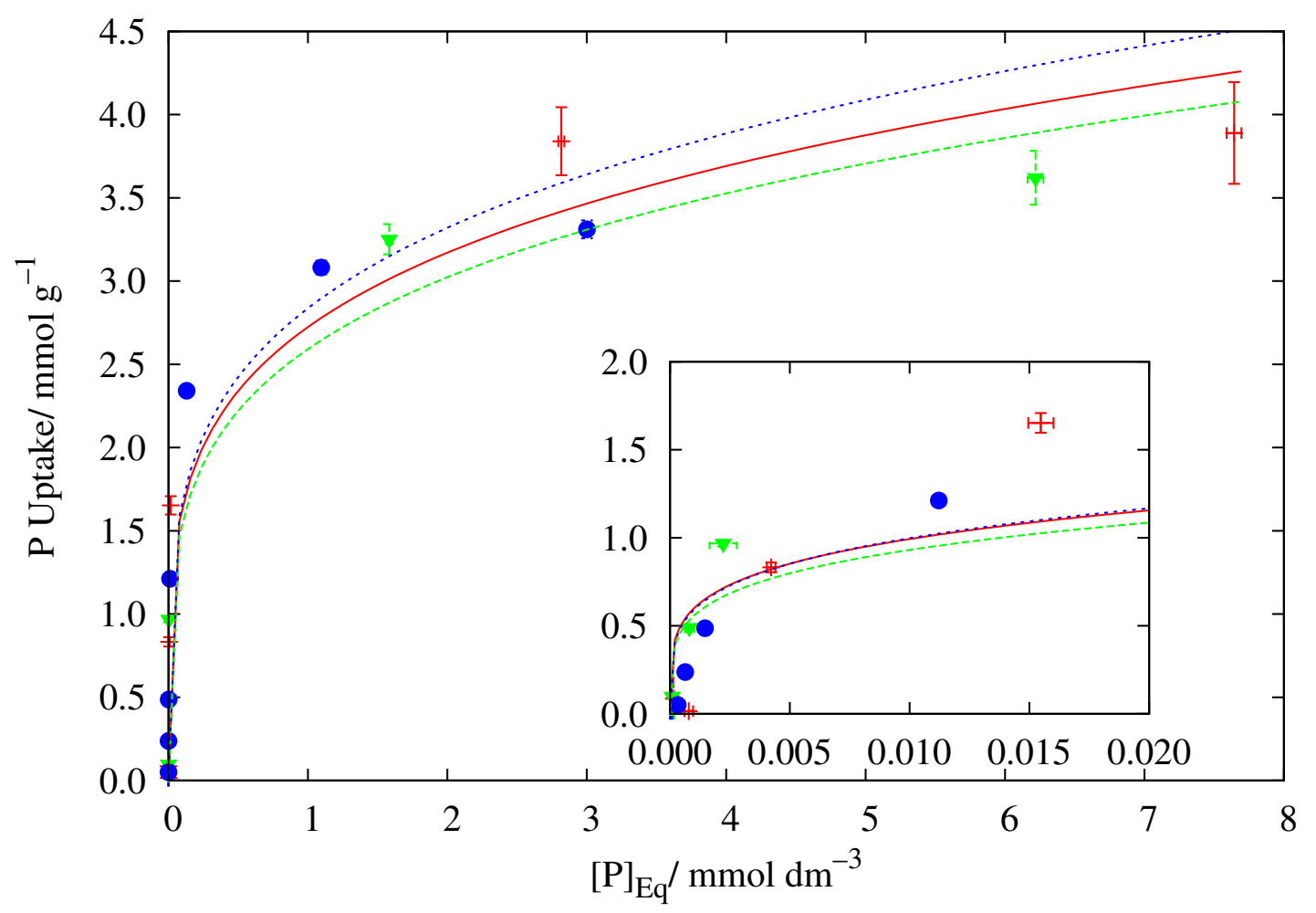

(b) Freundlich isotherm model.

Figure 5.2 First part of Figure 5.2. (Figure continued over page.) 


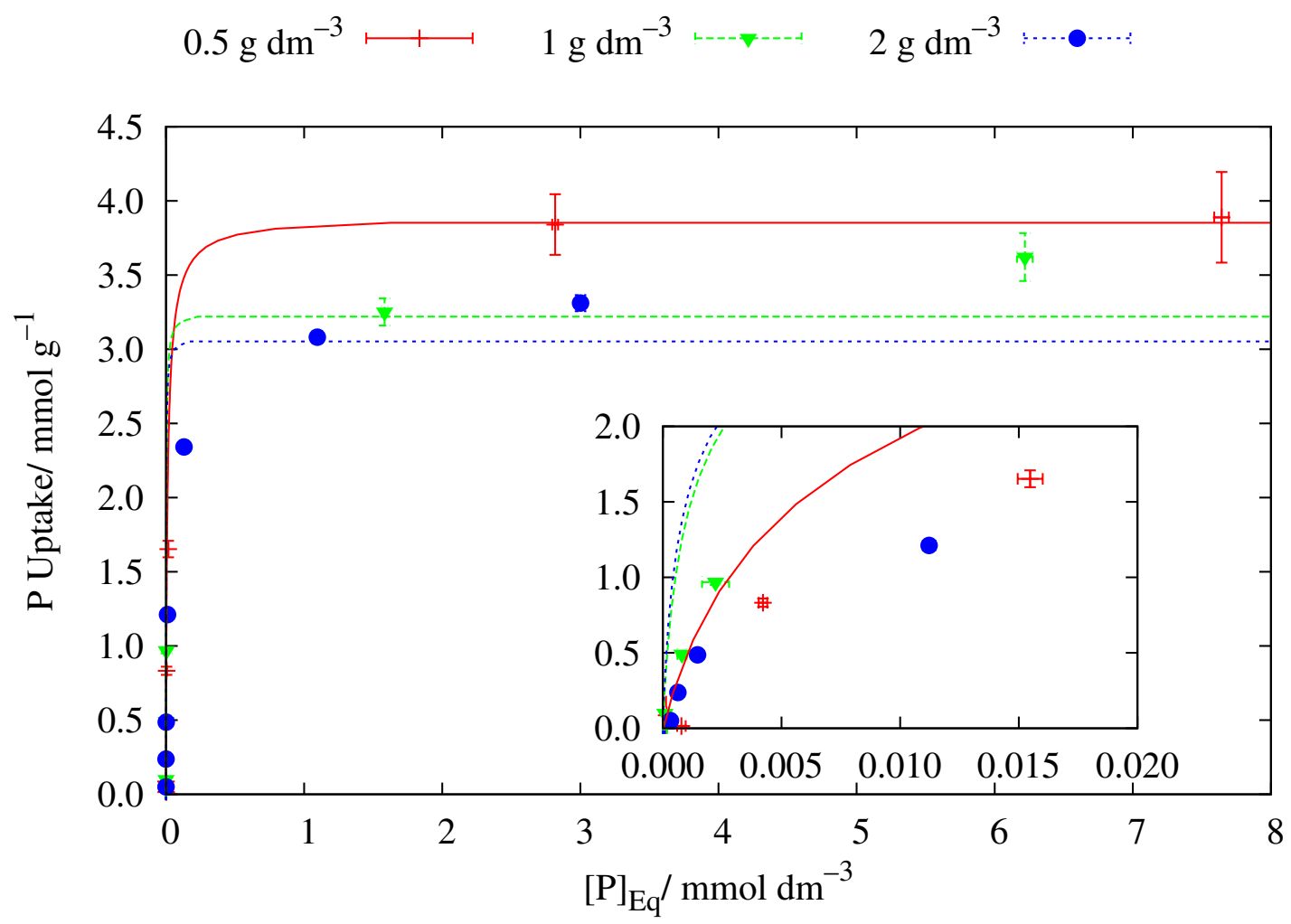

(c) Frumkin isotherm model.

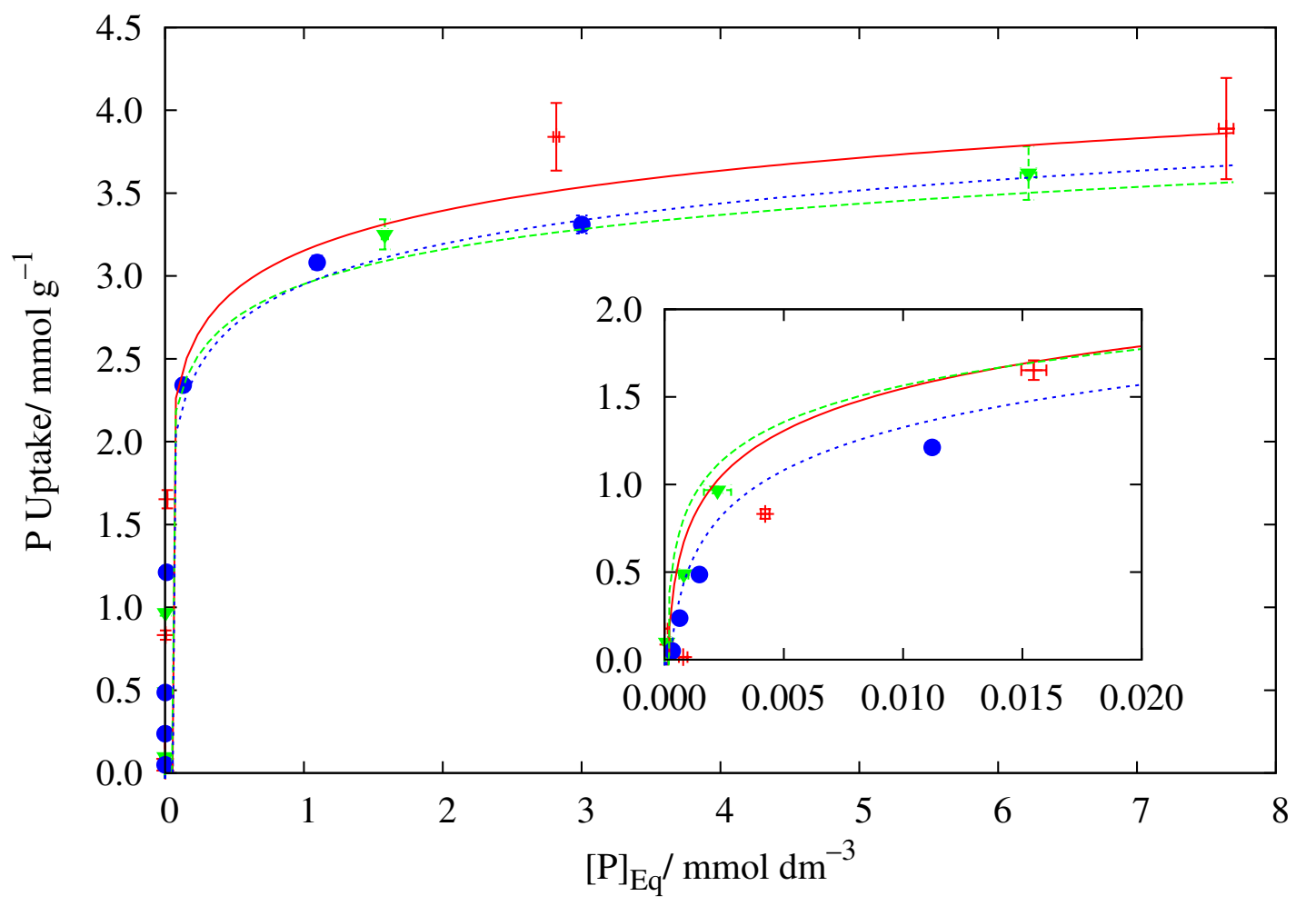

(d) Tempkin isotherm model.

Figure 5.2 Phosphate sorption at various $\mathrm{NCS}-\mathrm{Fe}_{3} \mathrm{O}_{4}$ loadings, with the data modelled by different isotherms. The experimental data is represented by points, the isotherm models are shown with lines: (a) the Langmuir isotherm; (b) the Freundlich isotherm; (c) the Frumkin isotherm; (d) the Tempkin isotherm. 
The data (Figure 5.2(a) and Table 5.2) obtained from the experiments conducted at sorbent loadings of $0.5 \mathrm{~g} \mathrm{dm}^{-3}$ were readily modelled by the Langmuir isotherm; the model having an $R^{2}$ value in excess of 0.995 . Attempts were also made to model the data by the Freundlich, Frumkin, or Tempkin isotherms. However $R^{2}$ values obtained were on the order of 0.94-0.95 and as such were less representative of the system than the Langmuir model. Isotherm experiments conducted with sorbent applied at increased loadings of $1 \mathrm{~g} \mathrm{dm}^{-3}$ or $2 \mathrm{~g} \mathrm{dm}^{-3}$ (dry basis) exhibited a lesser degree of conformity to the Langmuir isotherm, with $R^{2}$ values of 0.991 and 0.979 respectively. Data obtained from these experiments were not consistent with data obtained from the earlier experiment. The phosphate uptake attained by the silicate decreased (on a per mass basis) as the silicate loading increased. It was initially assumed that a model other than the Langmuir isotherm would provide a more appropriate fit to the data, as the other models attempt to account for the non-equivalence of sorption sites within the system, something that the Langmuir model does not inherently account for.

The results of the modelling with these additional isotherms can be seen in Figures 5.2(b)(d). None of these isotherms provide the same degree of conformity to the data as was obtained with the Langmuir model at the lower sorbent loading. This may be accounted for by one of several reasons:

- The most likely reason for this behaviour is that the system failed to reach thermodynamic equilibrium. As can be seen from Figure 5.2, at the $0.5 \mathrm{~g} \mathrm{dm}^{-3}$ sorbent loading saturation of the sorbent is achieved, as evidenced by the increase in equilibrium phosphate concentration not resulting in an increase in phosphate uptake. Similar behaviour is not obtained with the systems at loadings of $1 \mathrm{~g} \mathrm{dm}^{-3}$ and $2 \mathrm{~g} \mathrm{dm}^{-3}$. As can be seen from the figures, the sorption capacity does not reach a plateau. Instead, an increase in the equilibrium phosphate concentration continues to result in an increase in the phosphate uptake.

- The molybdenum blue method used for colourmetric determination of phosphate concentration suffers positive interference from the presence of silica, as the molybdate may react to form either a molybdophosphate complex of a molybdosilicate 
complex. It has previously been demonstrated that removal of calcium from the NCS material (typically by leaching with acid) also results in a silicate release to solution. ${ }^{22}$ While attempts were made to correct for possible interference by silicate in the phosphate determination (the colourmetric response in the absence of phosphate was used to provide a baseline during analysis), it is possible that insufficient correction was applied. This would lead to an increase in observed phosphate concentration and, hence, a decrease in the apparent phosphate sorption. This problem would be exaggerated at higher NCS concentrations.

- In the case of the Frumkin isotherm (Figure 5.2(c)), the isotherm model was fitted to the experimental data in a slightly different fashion. It has previously been noted that with the Frumkin isotherm (Equation (1.14)) $\theta$ is not a function of $\left[\mathrm{P}_{E q}\right]$ as it is for the other isotherm models. That is to say that for a given value of $\left[\mathrm{P}_{E q}\right]$ the equation may provide more than one possible value of $\theta$. For this reason, the experimental data were modelled by a function (as presented in (5.3)) very similar to Equation (1.13):

$$
[\mathrm{P}]_{E q}=\frac{n}{K\left(n_{\infty}-n\right)} \exp \left(-A \frac{n}{n_{\infty}}\right)
$$

In Equation (5.3), $[\mathrm{P}]_{E q}$ is treated as a function of $n$. In doing so, the method of calculating $R^{2}$ changes, being based on a variation in $[\mathrm{P}]_{E q}$ instead of $n$. The different method of modelling the experimental data may account for the poorer fit to the Frumkin isotherm when compared to the Langmuir isotherm. Theoretically, the Frumkin isotherm should never be a poorer model than the Langmuir isotherm, as when $A=0$, the two isotherms are identical.

\subsubsection{Sorption Capacity}

Although it is known that the primary crystalline phase formed in the sorption of phosphate by NCS is brushite, ${ }^{34}$ the $\mathrm{Fe}_{3} \mathrm{O}_{4}$-NCS composites sorbed too little phosphate relative to the amount of calcium present. In brushite, $\mathrm{CaHPO}_{4} \cdot 2 \mathrm{H}_{2} \mathrm{O}$, the Ca:P ratio is 1:1, but maximum phosphate sorption by NCS was attained when a Ca:P ratio of 1.4:1 was reached. The materials sorbed sorbed $5.1 \pm 0.5 \mathrm{mmol} \mathrm{P} \mathrm{g}^{-1} \mathrm{NCS}$ with this sorption 
Table 5.4 Parameters for isotherm models of phosphate sorption by $\mathrm{Fe}_{3} \mathrm{O}_{4}$-NCS composite material.

\begin{tabular}{lccr}
\hline \multirow{2}{*}{ Parameter } & \multicolumn{4}{c}{$\mathrm{Fe}_{\mathbf{3}} \mathbf{O}_{\mathbf{4}}$-NCS loading $\left(25\right.$ wt $\left.\% \mathbf{F e}_{\mathbf{3}} \mathbf{O}_{\mathbf{4}}\right) / \mathbf{g ~ d m}^{-\mathbf{3}}$} \\
\cline { 2 - 4 } & \multicolumn{4}{c}{$\mathbf{0 . 5}$} & $\mathbf{1 . 0}$ & $\mathbf{2 . 0}$ \\
\cline { 2 - 4 } & \multicolumn{4}{c}{ Langmuir Isotherm } \\
\hline$n_{\infty}{ }^{a}$ & 3.88 & 3.45 & 3.10 \\
$K^{b}$ & 51.3 & 168 & 51.9 \\
$R^{2}$ & 0.996 & 0.991 & 0.979 \\
\hline
\end{tabular}

\section{Freundlich Isotherm}

\begin{tabular}{lrrr}
\hline$n_{\infty} a$ & 2.72 & 2.59 & 2.84 \\
$C_{2}$ & 4.56 & 4.50 & 4.40 \\
$R^{2}$ & 0.942 & 0.962 & 0.934 \\
\hline
\end{tabular}

\section{Frumkin Isotherm}

\begin{tabular}{lrrr}
\hline$n_{\infty}$ & 3.89 & 3.25 & 3.08 \\
$A$ & 1.18 & 3.14 & 3.43 \\
$K$ & 50.0 & 50.2 & 50.1 \\
$R^{2 c}$ & 0.894 & -0.154 & -0.137 \\
\hline
\end{tabular}

\section{Tempkin Isotherm}

\begin{tabular}{lrrr}
\hline$n_{\infty} C_{1}$ & 0.350 & 0.301 & 0.353 \\
$C_{2}$ & $8.30 \times 10^{3}$ & $1.81 \times 10^{4}$ & $4.30 \times 10^{3}$ \\
$R^{2}$ & 0.950 & 0.960 & 0.986 \\
\hline
\end{tabular}

${ }^{a}$ Presented in dimensions of $\mathrm{mmol} \mathrm{g}^{-1}$.

${ }^{b}$ Presented in dimensions of $\mathrm{dm}^{3} \mathrm{mmol}^{-1}$

${ }^{c}$ Calculated from variation in $\left[\mathrm{P}_{E q}\right]$, not $\mathrm{P}$ uptake. 
decreasing proportionally as $\mathrm{Fe}_{3} \mathrm{O}_{4}$ was incorporated into the structure. By comparison, NCS contains $7.1 \mathrm{mmol} \mathrm{Ca} \mathrm{g}^{-1}$. This indicates that, either not all calcium present in the NCS is coordinated to phosphate, or that a calcium phosphate phase with a $\mathrm{Ca}: \mathrm{P}$ ratio greater than 1:1 is formed. Due to the saturation of NCS with phosphate, it is probable that partial formation of the more thermodynamically stable apatite phase, with a Ca:P ratio of $1.67: 1$, occurs.

\subsubsection{Kinetic Studies}

The sorption of $\mathrm{H}_{2} \mathrm{PO}_{4}^{-}$is modelled well by a second-order kinetic model with respect to phosphate (Figure 5.3 and Table 5.5), with an $R^{2}$ value of 0.997. By comparison, the first-order kinetic model provided an $R^{2}$ value of 0.984 . As these values are both relatively high the reaction cannot be definitively described to a second-order model, although it is more likely than the first-order one. During the 60 minutes for which data were recorded, the concentration of $\mathrm{H}_{2} \mathrm{PO}_{4}^{-}$decreased from $0.99 \pm 0.01 \mathrm{mmol} \mathrm{dm}^{-3}$, to $0.138 \pm 0.001 \mathrm{mmol} \mathrm{dm}^{-3}$. This is in agreement with the second-order model reported by Southam $^{34}$ for the non-magnetic NCS material. Southam reported two distinct processes occurring in the reaction of NCS with $\mathrm{H}_{2} \mathrm{PO}_{4}^{-}$: An initial second order process (with respect to $\mathrm{H}^{+}$) occurring in the first 30 minutes, attributed to the reaction of NCS with $\mathrm{H}^{+}$, leading to an increase in the $\mathrm{pH}$ of the solution, followed by a second second-order process (with respect to phosphate) in which phosphate (predominantly $\mathrm{HPO}_{4}^{2-}$ ) reacts with NCS precipitating calcium phosphate phases such as brushite $\left(\mathrm{CaHPO}_{4} \cdot 2 \mathrm{H}_{2} \mathrm{O}\right)$.

Table 5.5 Rate data for phosphate sorption by $\mathrm{Fe}_{3} \mathrm{O}_{4}-\mathrm{NCS}$ composite.

\begin{tabular}{lrr}
\hline Parameter & First Order $^{a}$ & Second Order $^{b}$ \\
\hline$k$ & 0.332 & 0.774 \\
{$[\mathrm{P}]_{0, \text { Calc. }}{ }^{c}$} & 0.988 & 0.989 \\
{$[\mathrm{P}]_{\Delta}$} & 0.795 & 0.848 \\
$R^{2}$ & 0.984 & 0.997 \\
\hline
\end{tabular}

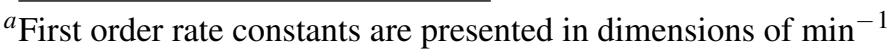

${ }^{b}$ Second order rate constants are presented in dimensions of $\mathrm{dm}^{3} \mathrm{mmol}^{-1} \mathrm{~min}^{-1}$.

${ }^{c}$ Concentrations are presented in dimensions of $\mathrm{mmol} \mathrm{dm}^{-3}$. 


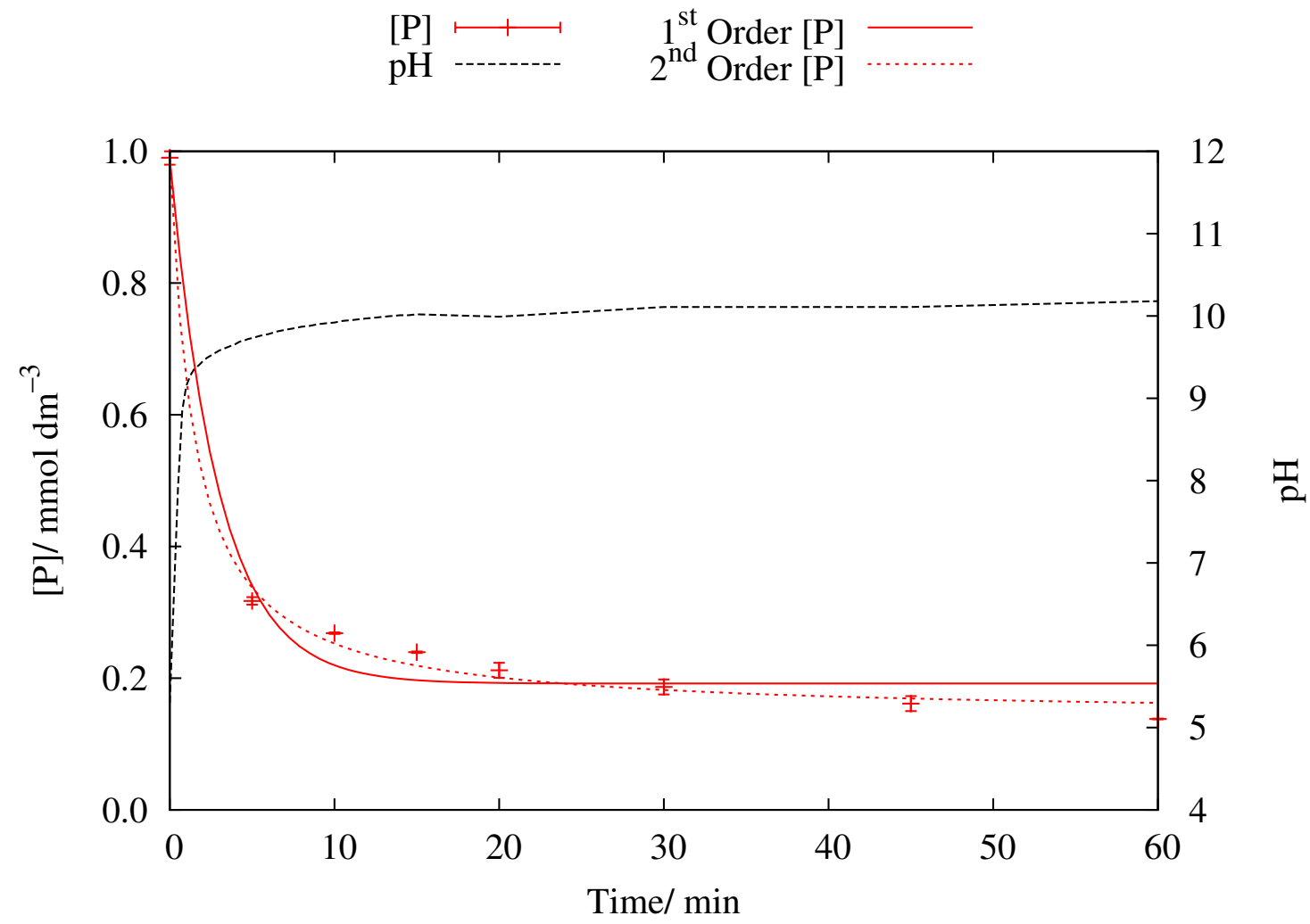

Figure 5.3 Sorption of $\mathrm{H}_{2} \mathrm{PO}_{4}^{-}$by $\mathrm{Fe}_{3} \mathrm{O}_{4}$-NCS composite, 25 wt $\% \mathrm{Fe}_{3} \mathrm{O}_{4}$. NCS filter cake applied at a loading of $1 \mathrm{~g} \mathrm{dm}^{-3}$ (dry basis). Data points represent the measured phosphate concentration; the solid red line shows a first order kinetic model; the dashed red line shows a second order kinetic model. $\mathrm{pH}$ values have not been modelled. 


\subsubsection{Wastewater Treatment}

Attempts were made to use the $\mathrm{Fe}_{3} \mathrm{O}_{4}$-NCS material to sorb phosphate from a whey-type wastewater obtained from a local dairy factory near Karlsruhe. Analysis of the phosphorus content of the wastewater provided phosphate contents of: $1.56 \pm 0.01 \mathrm{mmol} \mathrm{dm}{ }^{-3} \mathrm{DRP}$ (dissolved reactive phosphorus), $1.84 \pm 0.01 \mathrm{mmol} \mathrm{dm}^{-3} \mathrm{TRP}$ (total reactive phosphorus) and $2.11 \pm 0.01 \mathrm{mmol} \mathrm{dm}{ }^{-3} \mathrm{TP}$ (total phosphorus), the latter by $\mathrm{HNO}_{3}$ digestion. The dairy waste was also found to contain approximately 5-10\% settleable solids (by volume).

Although attempts were made to measure both an sorption isotherm for the waste solution and to acquire kinetic data for the phosphate uptake, filtration of the dairy waste was found to be impractical on a reasonable time scale. Some component within the waste (presumed to be fat) was found to block the $0.45 \mu \mathrm{m}$ membranes used for filtration. In most cases insufficient sample could be obtained for phosphate analysis (the DRP analysis of the raw waste, given above, was obtained only after significant dilution).

Due to the difficulties encountered in filtering the wastewater, attempts to characterise the sorption qualities of the material with regard to the dairy waste were abandoned.

\subsubsection{Continuous Uptake Studies}

The results of the continuous uptake studies of $\mathrm{H}_{2} \mathrm{PO}_{4}^{-}$are presented in Figure 5.4 and Table 5.4. Figures 5.4(a) and 5.4(b) show the results of experiments performed with a larger, $600 \mathrm{~cm}^{3}$ column, whereas 5.4(c) displays the results from the use of a smaller $50 \mathrm{~cm}^{3}$ column.

As can be seen from the figures, the smaller column achieved saturation, with the phosphate effluent concentration eventually exceeding the influent concentration. A maximum sorption capacity of $0.82 \pm 0.06 \mathrm{mmol} \mathrm{P} \mathrm{g}^{-1}$ was calculated for the smaller column. This is significantly less that the $3.9 \mathrm{mmol} \mathrm{P} \mathrm{g}^{-1}$ as measured in the isotherm sorption experiments. The lower sorption capacity is partially explained by noting that nearly half the mass of sorbent was lost from the column in the effluent stream. The 


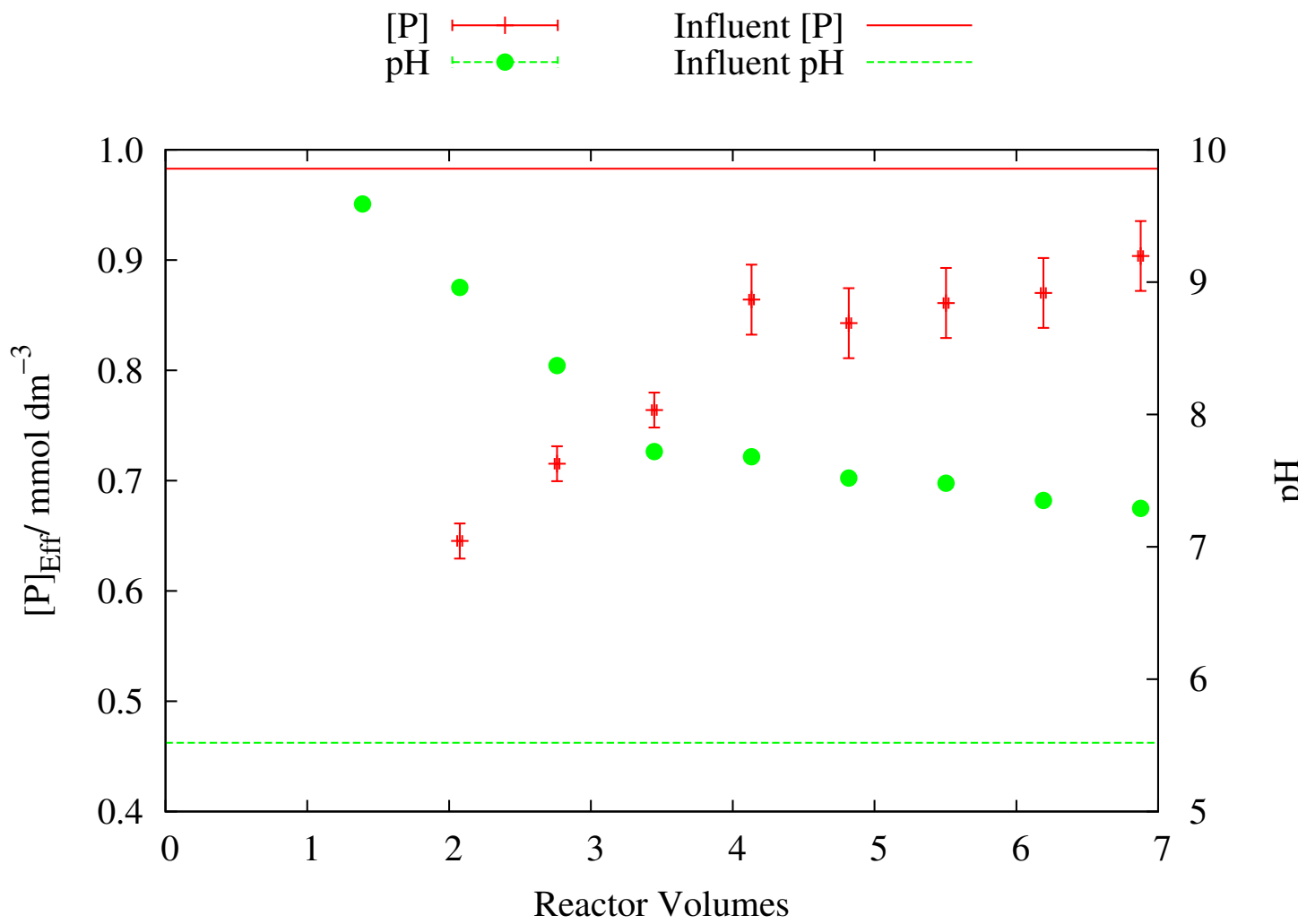

(a)

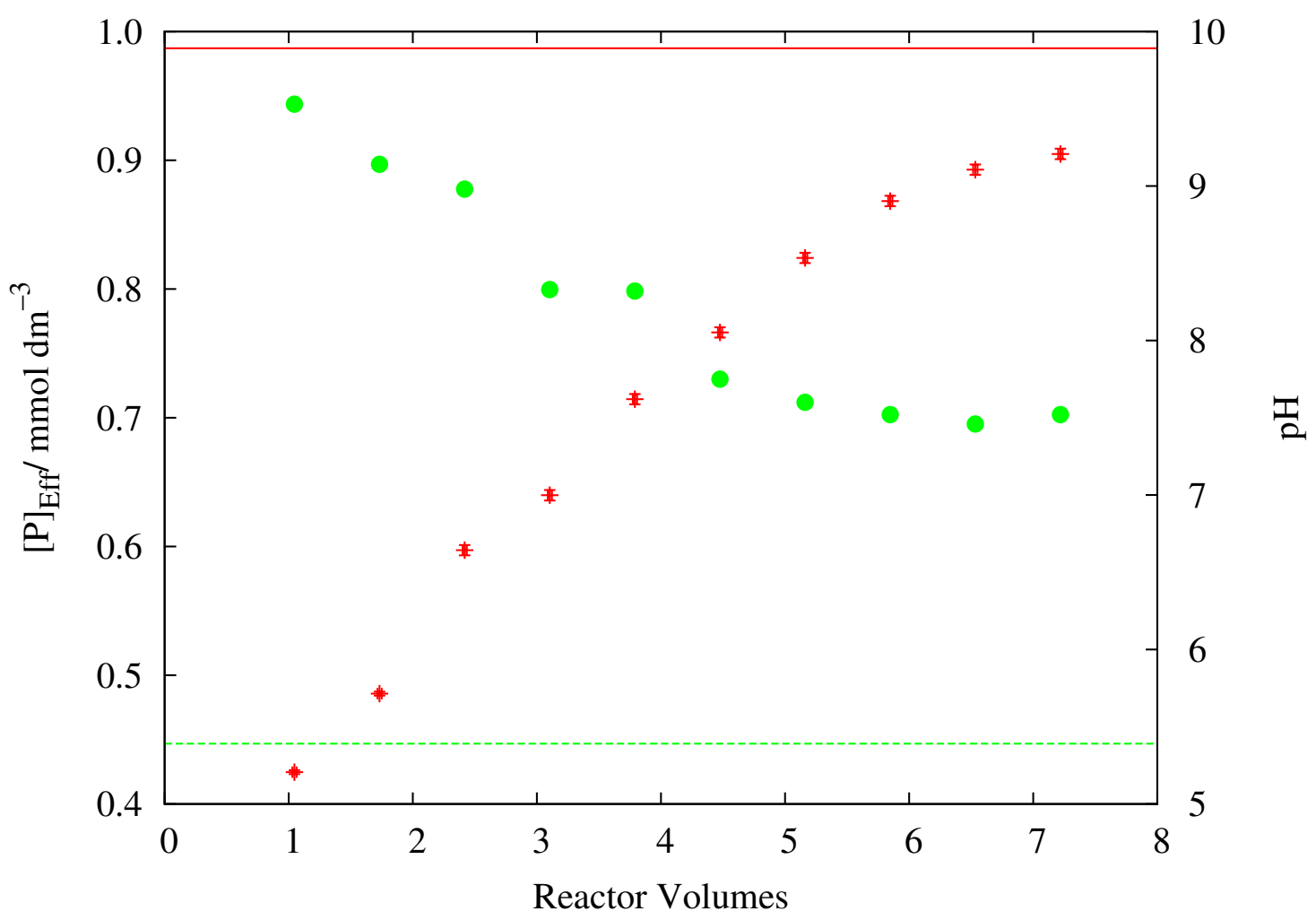

(b)

Figure 5.4 First part of Figure 5.4. (Figure continued over page.) 


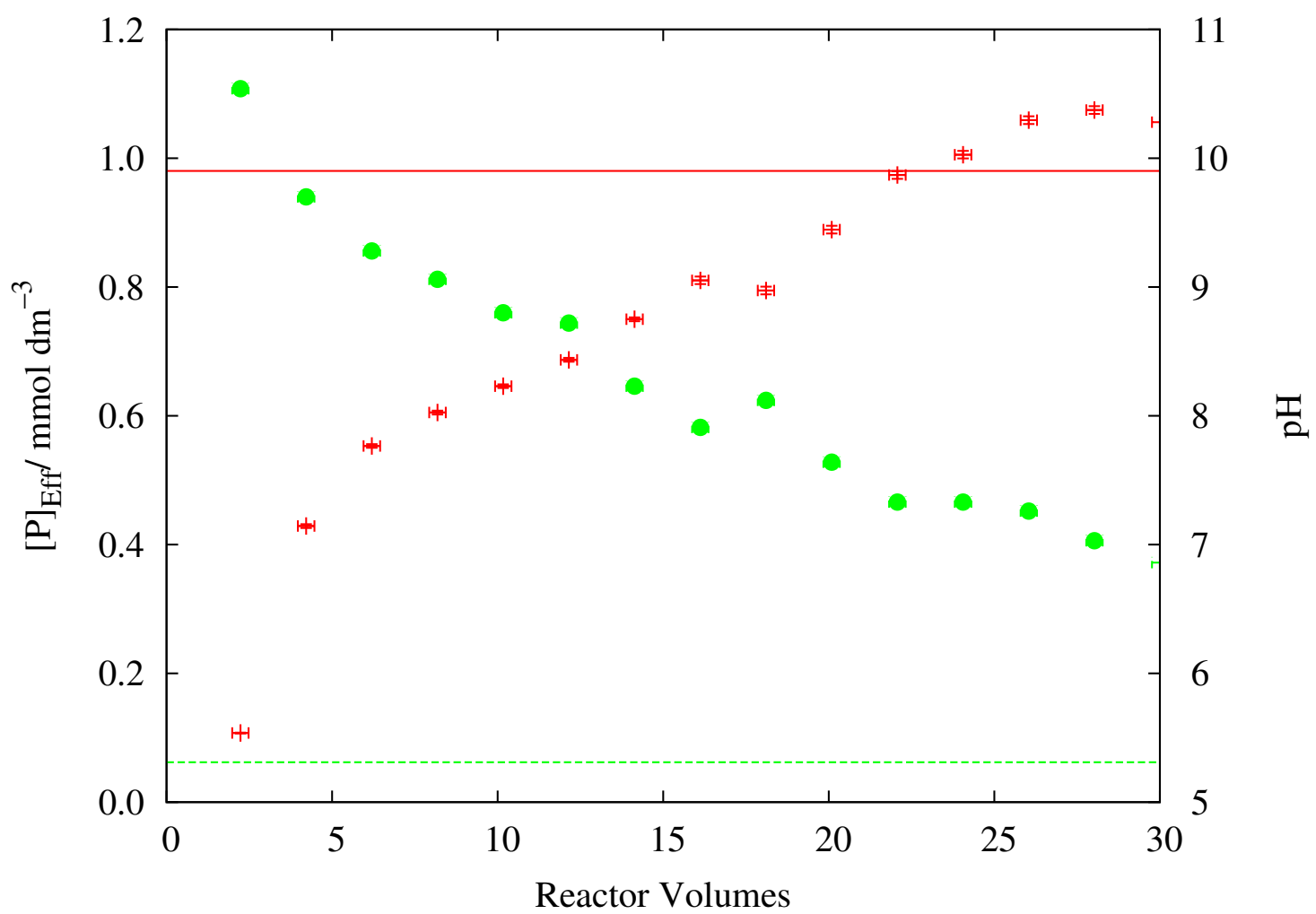

(c)

Figure 5.4 Column-based continuous uptake of phosphate (as $\mathrm{H}_{2} \mathrm{PO}_{4}^{-}$by $\mathrm{Fe}_{3} \mathrm{O}_{4}-\mathrm{NCS}$ composite. Figures (a) and (b) show results from experiments with a $600 \mathrm{~cm}^{3}$ column, whereas Figure (c) shows data from a $50 \mathrm{~cm}^{3}$ column. Data points represent the effluent $\mathrm{pH}$ and phosphate concentration; the lines show the influent conditions. 


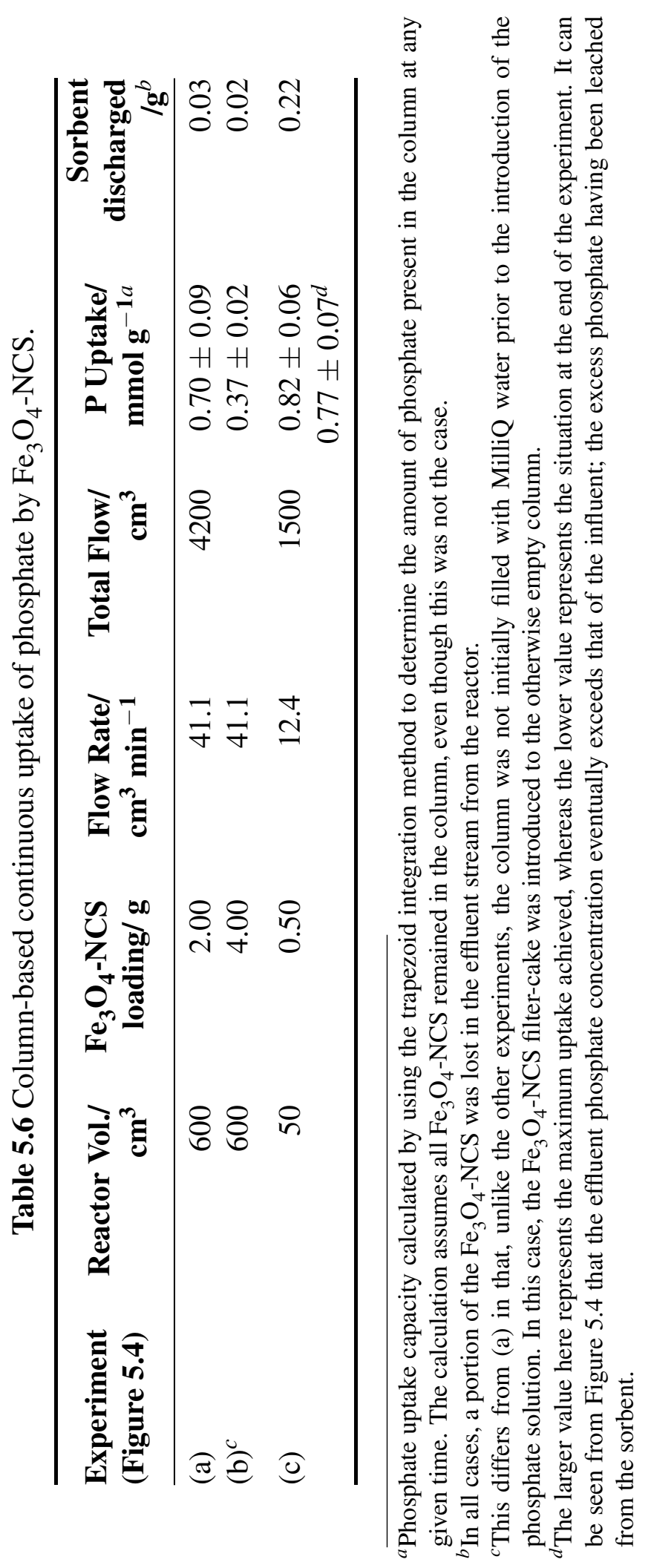


$0.82 \mathrm{mmol} \mathrm{P} \mathrm{g}^{-1}$ figure calculated above is based on the assumption that all sorbent remained in the column.

By comparison, the experiments conducted with the larger column did not reach saturation in that the measured phosphate effluent concentration was less than the influent concentration for the duration of the experiment. These experiments exhibited very similar total phosphate sorption $(1.4 \pm 0.2 \mathrm{mmol} \mathrm{P}$ for Figure 5.4(a) and $1.48 \pm 0.08 \mathrm{mmol} \mathrm{P}$ for Figure 5.4(b), despite one experiment having double the mass of NCS present. This leads to the assumption that the system in this case was rate limited by the amount of phosphate present.

For the smaller column, a comparatively large fraction of the sorbent was removed in the effluent stream (approximately $44 \%$ of the initial mass of sorbent). Contrasting this, almost no sorbent was removed from the larger column. This may be explained by examining the solution velocity in the column. The smaller column had an internal diameter of $20 \mathrm{~mm}$, a cross-section of $3.14 \mathrm{~cm}^{2}$. At the flow rate used, this equates to an upward velocity of $3.9 \mathrm{~cm} \mathrm{~min}^{-1}$. With the larger column, the diameter varied (see Figure 2.1). At the exit point of the column, the internal diameter was $120 \mathrm{~mm}$, a crosssecion of $117 \mathrm{~cm}^{2}$. This means that when the effluent exited the larger column, it was only moving at a rate of $0.35 \mathrm{~cm} \mathrm{~min}^{-1}$ (approximately one eleventh the velocity present in the smaller column). During operation of the large column, the $\mathrm{Fe}_{3} \mathrm{O}_{4}$-NCS material remained dispersed in the lower half of the column. The internal column diameter at the upper edge of this $\mathrm{Fe}_{3} \mathrm{O}_{4}$-NCS layer was estimated as $90 \mathrm{~mm}$. The upwards velocity of the fluid in the column at this point may be calculated as $0.6 \mathrm{~cm} \mathrm{~min}{ }^{-1}$.

As can be seen from the images of the experimental set-up (Figure 5.5), the $\mathrm{Fe}_{3} \mathrm{O}_{4}$-NCS was dispersed throughout the small column (Figure 5.5(b)) to a much greater degree than in the larger column (Figure 5.5(d)). The majority of the sorbent lost from the column was removed in the initial few column volumes of effluent. Figure 5.5(c) demonstrates this. This image shows the discolouration of the $0.45 \mu \mathrm{m}$ membranes used to filter effluent subsamples subsequent to their collection. These samples were later analysed to provide data for the first five points shown in Figure 5.4(c). It can be seen that the $\mathrm{Fe}_{3} \mathrm{O}_{4}$-NCS was 
initially lost at a significant rate (as indicated by the black sorbent trapped in the leftmost filter cartridge, labelled 'A', in Figure 5.5(c)). By the time the fifth subsample was taken very little sorbent was being lost (the rightmost filter cartridge, labelled ' $E$ ', shows almost no discolouration). 

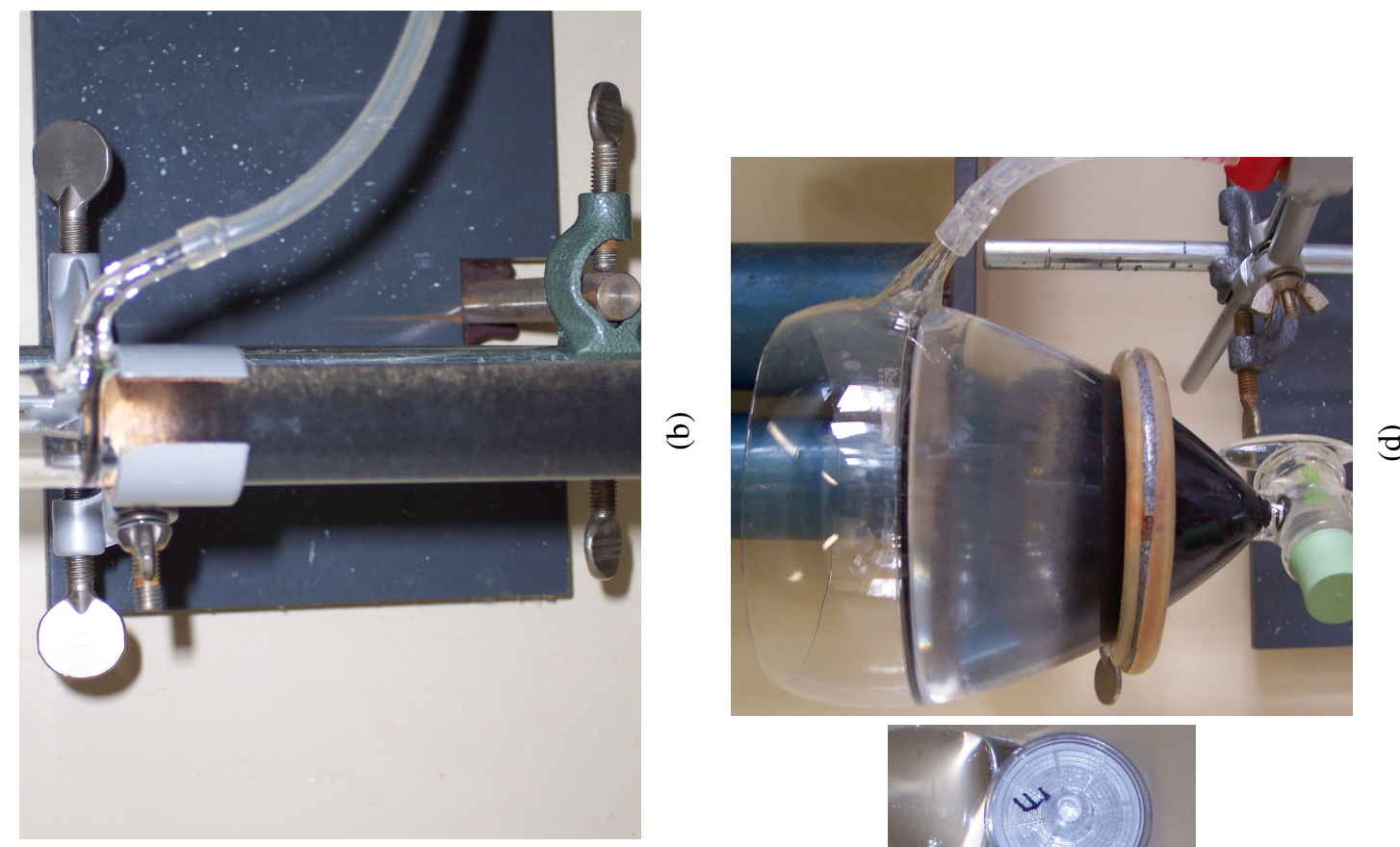

:
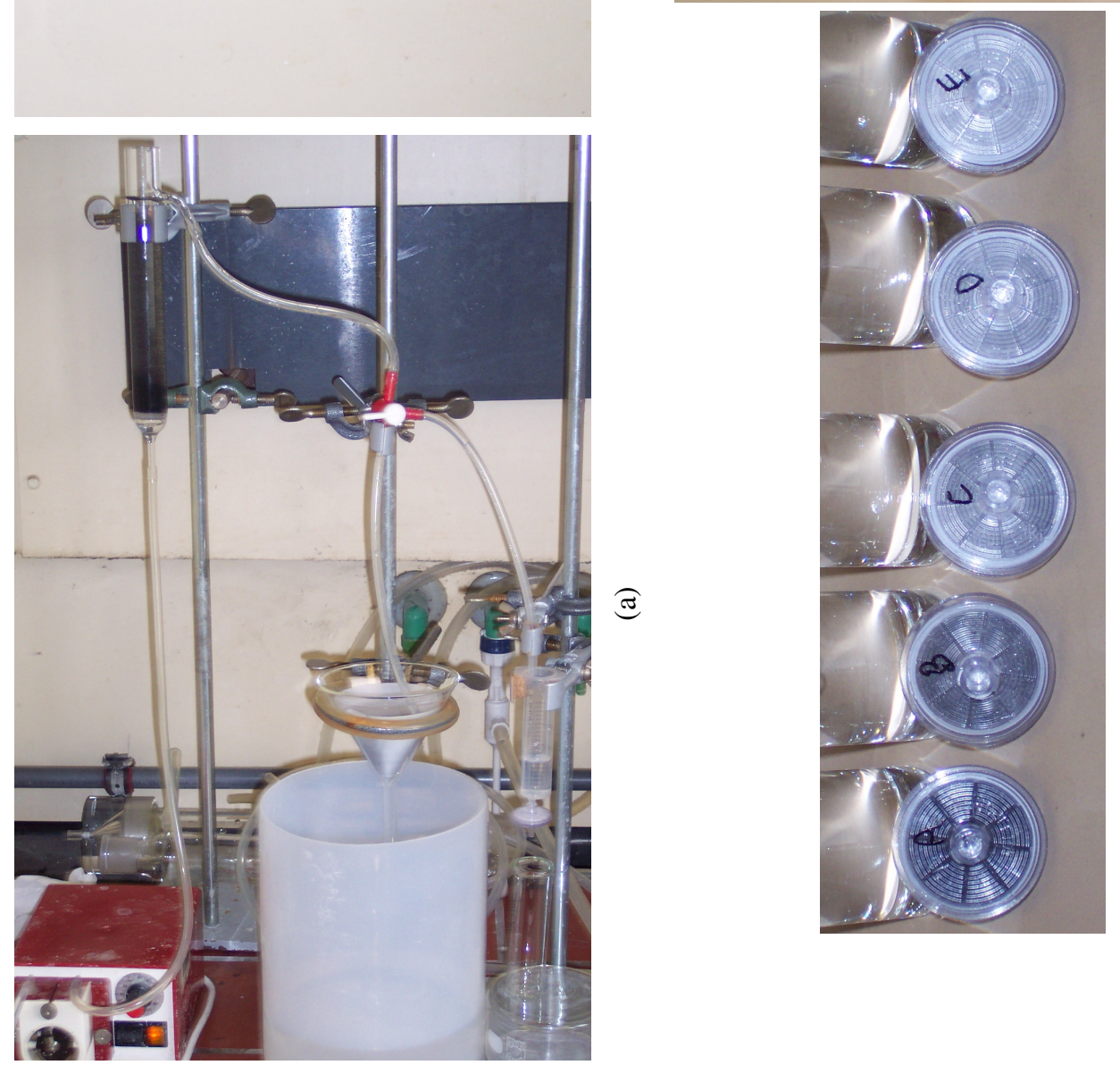

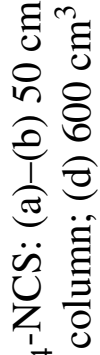

$0^{+\infty}{ }^{+} \overline{0}$

II

e

ล

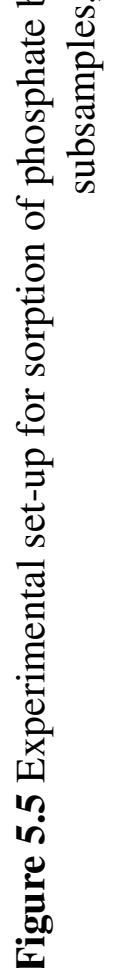




\subsection{Conclusions}

It was found that the $\mathrm{Fe}_{3} \mathrm{O}_{4}$-NCS composite was a suitable sorbent material for phosphate. The sorption capacity for the materials was found to be dependant on the quantity of NCS present. $\mathrm{As} \mathrm{Fe}_{3} \mathrm{O}_{4}$ was introduced into the composite material, the sorption capacity decreased accordingly with $\mathrm{Fe}_{3} \mathrm{O}_{4}$ accounting for very little of the observed phosphate sorption. For the unmodified NCS, an sorption capacity of $5.1 \pm 0.5 \mathrm{mmol} \mathrm{P} \mathrm{g}$ was recorded for phosphate as the $\mathrm{H}_{2} \mathrm{PO}_{4}^{-}$ion; sorption capacities of NCS for other forms of phosphate were not determined. While this is significantly higher than the $1.94 \mathrm{mmol} \mathrm{P} \mathrm{g}^{-1}$ sorption capacity previously reported by Southam ${ }^{34}$ for sorption of $\mathrm{H}_{2} \mathrm{PO}_{4}^{-}$on to NCS, the amount of phosphate sorbed is comparable to the amount of calcium present in the material.

When isotherm measurements were conducted with the $\mathrm{Fe}_{3} \mathrm{O}_{4}-\mathrm{NCS}$ composite material ( $25 \mathrm{wt} \% \mathrm{Fe}_{3} \mathrm{O}_{4}$ ) present at a loading of $0.5 \mathrm{~g} \mathrm{dm}^{-3}$, the system exhibited good conformity to the Langmuir isotherm. An $R^{2}$ value of 0.996 was calculated for the model, having a calculated maximum sorption capacity, $n_{\infty}$, of $3.88 \mathrm{mmol} \mathrm{P} \mathrm{g}^{-1}$, with the parameter $K$ having a value of $51.3 \mathrm{dm}^{3} \mathrm{mmol}^{-1}$. As the loading of $\mathrm{Fe}_{3} \mathrm{O}_{4}-\mathrm{NCS}$ was increased to $2.0 \mathrm{~g} \mathrm{dm}^{-3}$, the $R^{2}$ value decreased to 0.979 for the Langmuir isotherm model. At the highest loading, the Tempkin isotherm was found to more accurately model the data, with an $R^{2}$ value of 0.986 . It was also noted that the phosphate sorption capacity (on a per gram basis) decreased as the sorbent loading was increased (from $3.9 \pm 0.3 \mathrm{mmol} \mathrm{P} \mathrm{g}^{-1}$ with a $0.5 \mathrm{~g} \mathrm{dm}^{-3}$ sorbent concentration, to $3.31 \pm 0.05 \mathrm{mmol} \mathrm{P} \mathrm{g}-1$ a a $2.0 \mathrm{~g} \mathrm{dm}^{-3}$ sorbent concentration. The reduction in the observed sorption capacity was attributed to the system not attaining equilibrium within 24 hours at the higher NCS concentrations. This was evidenced by the phosphate sorption not reaching saturation for the higher sorbent concentrations. The sorption capacity did not plateau with higher solution phosphate concentrations. Instead, it continued to increase.

The sorption of $\mathrm{H}_{2} \mathrm{PO}_{4}^{-}$by $\mathrm{Fe}_{3} \mathrm{O}_{4}$-NCS over a 60 minute time period was adequately described by a second-order kinetic model, having a rate constant of $k=0.774 \mathrm{dm}^{3} \mathrm{mmol}^{-1}$ $\min ^{-1}$, with an $R^{2}$ value of 0.997 . Similar behaviour has been observed by Southam ${ }^{34}$, 
who attributed this to an acid/base reaction between the acidic $\mathrm{H}_{2} \mathrm{PO}_{4}^{-}$solution and the basic NCS.

Continuous uptake studies were performed in upflow mode using two different reactor sizes and shapes, a small $50 \mathrm{~cm}^{3}$ cylindrical reactor and a large $600 \mathrm{~cm}^{3}$ conical reactor. Under the conditions employed with the smaller column, the $\mathrm{Fe}_{3} \mathrm{O}_{4}$-NCS was found to sorb a maximum of $0.82 \pm 0.06 \mathrm{mmol} \mathrm{P} \mathrm{g} \mathrm{g}^{-1}$ before the material reached saturation, significantly lower than the maximum sorption capacity of $3.9 \pm 0.3 \mathrm{mmol} \mathrm{P} \mathrm{g}^{-1}$ attained in the isotherm measurements. This uptake accounted for only $37 \%$ of the phosphate passed through the column. The smaller column also lost $44 \%$ of the $\mathrm{Fe}_{3} \mathrm{O}_{4}$-NCS in the effluent stream. By comparison, the $\mathrm{Fe}_{3} \mathrm{O}_{4}$-NCS sorbent used in the larger column experiments also sorbed between $33-35 \%$ of the phosphate from the influent stream. The system did not reach saturation however. In the larger column, for which two different loadings of $\mathrm{Fe}_{3} \mathrm{O}_{4}$-NCS sorbent were used, the total amount of phosphate sorbed from the solution did not depend on the amount of sorbent present; doubling the mass of sorbent did not alter the amount of phosphate sorbed. Therefore, the system is rate-limited by the contact time between the sorbent and phosphate solution. 


\section{Chapter 6}

\section{Rhenium Sorption by Polyaniline-NCS Composite}

\section{Materials}

\subsection{Introduction}

Technetium is often a significant component of nuclear waste streams. The ${ }^{99} \mathrm{Tc}$ isotope is not recovered during typical reprocessing procedures, such as flocculation or precipitation, primarily due to the solubility of the pertechnetate ion $\mathrm{TcO}_{4}^{-}$. It has been reported that the waste stream from a typical reprocessing plant may contain ${ }^{99} \mathrm{TcO}_{4}^{-}$at concentrations of up to $1 \mathrm{mmol} \mathrm{dm}{ }^{-3} \cdot{ }^{164,165}$ The ${ }^{99} \mathrm{Tc}$ isotope decays via $\beta$ emission to ${ }^{99} \mathrm{Ru}$; the half life of this process being on the order of $2.1 \times 10^{5}$ years. ${ }^{165}$ While this is insignificant on a geological time scale, it is impractical to store the technetium waste at such a dilute concentration until it has decayed to a level at which it poses no radiological threat. As such, treatment methods are required to remove the technetium from this waste. Rhenium is in many ways chemically analogous to the the element technetium, more so than the other stable member of group 7, manganese. Rhenium compounds are often used in sorption studies as a model compound for technetium as rhenium is safer to handle, due to its non-radioactive nature.

\subsubsection{Polyaniline}

The conducting polymers are a class of organic polymer materials typified by the presence of extensive $\pi$-conjugation along the polymer backbone. ${ }^{166}$ Polyaniline is a rarity among the conducting polymers in that the nitrogen heteroatom is integral to the $\pi$-conjugation system. As such, changes to the degree of protonation of the nitrogen, or its oxidation state can significantly alter the degree of this conjugation. Altering the degree of conjugation can greatly perturb the electronic and hence spectrographic properties of the polymer. 
Polyaniline exhibits three readily accessible oxidation states: pernigraniline, a fully oxidised form, in which the nitrogen is present as an imine functionality; leucoemeraldine, the fully reduced form, in which the nitrogen is present as amine functionalities; and emeraldine, the partially oxidised form in which a mix of amine and imine functionalities are present. The standard reduction potential for the transition from pernigraniline salt to emeraldine salt has been reported as $0.75 \mathrm{~V}$. For the transition from emeraldine salt to leucoemeraldine base, the standard reduction potential is $0.30 \mathrm{~V} \cdot{ }^{29}$ Examples of these oxidation states are shown in Figure 6.1. In a form in which formal charges may be assigned to the molecule, the polymer has a four-aniline repeat unit, although it is thought that a three-aniline repeat unit may fully describe the electronic structure of polyaniline when computational modelling of the polymer is undertaken. ${ }^{167}$

During the synthesis of many organic polymer materials, acidic conditions are necessary to ensure proper polymerisation of the monomer material. As many of these syntheses are undertaken in aqueous conditions, the acidic conditions are also necessary to dissolve the monomer. For example, aniline $\left(\mathrm{C}_{6} \mathrm{H}_{5} \mathrm{NH}_{2}\right)$ is only sparingly soluble in water, whereas anilinium $\left(\mathrm{C}_{6} \mathrm{H}_{5} \mathrm{NH}_{3}^{+}\right)$is readily water soluble. ${ }^{29}$ The synthesis of polyaniline, for example, is believed to occur via the mechanism shown in Figure 6.2. Under acidic conditions, aniline monomers polymerise by oxidative addition at the para position, forming the traditional polyaniline structure. ${ }^{169}$ When the reaction conditions are such that the $\mathrm{pH}$ is greater than 3 , oxidation of aniline leads to the formation of diphenylhydrazine. ${ }^{170}$ Further oxidation can lead to the formation of oligomers containing $\mathrm{N}-\mathrm{N}$ bonds. These polyazanes exhibit significantly different spectroscopic and redox properties to polyaniline.

Many examples of conducting polymer composite materials exist in which the oxidation of the organic monomer is achieved subsequent to its sorption onto a silica ${ }^{171,172}$ or aluminosilicate ${ }^{173,174}$ support material. This method does pose a limitation on the framework material used: the material must be chemically inert to the reaction conditions for the oxidation process. Silica is nonreactive towards acid, and although acidic conditions are known to leach aluminium from aluminosilicates, the leaching reaction proceeds moderately slowly. ${ }^{175} \mathrm{NCS}$, by comparison, will rapidly degrade in acidic 


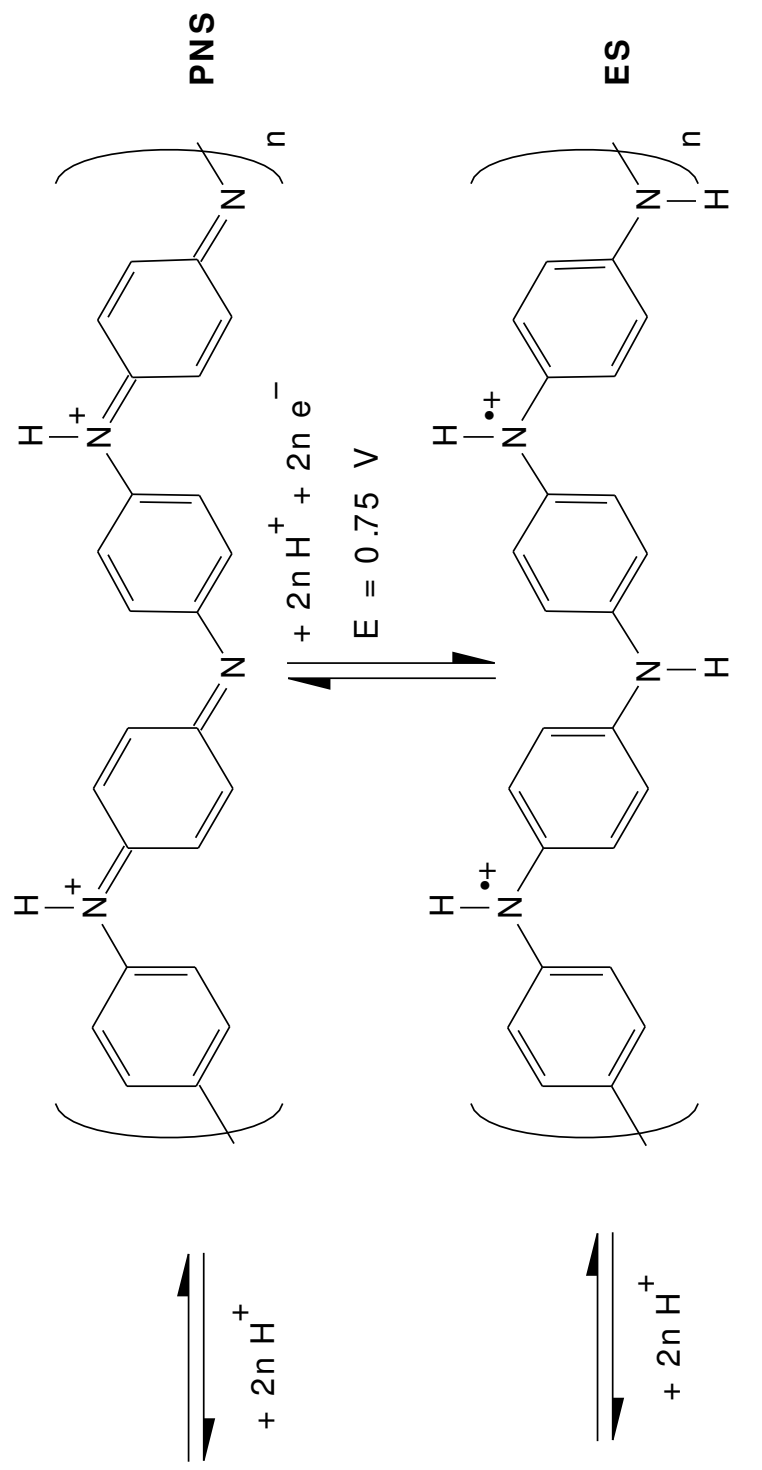

$\frac{m}{2}$
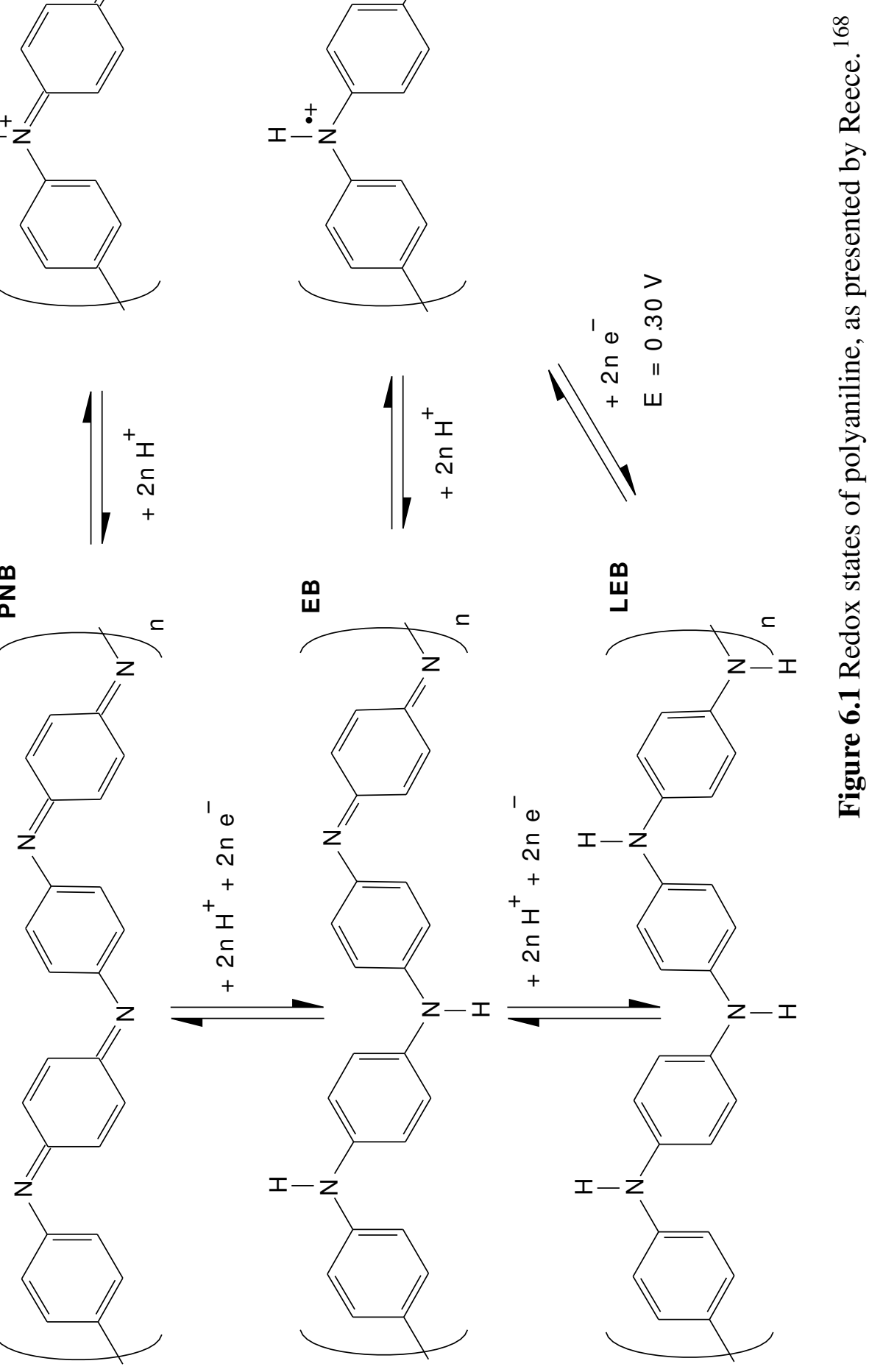

Щำ

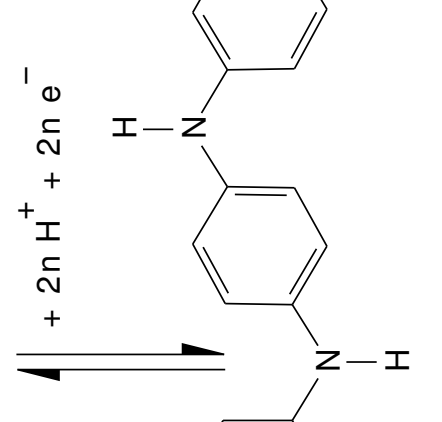




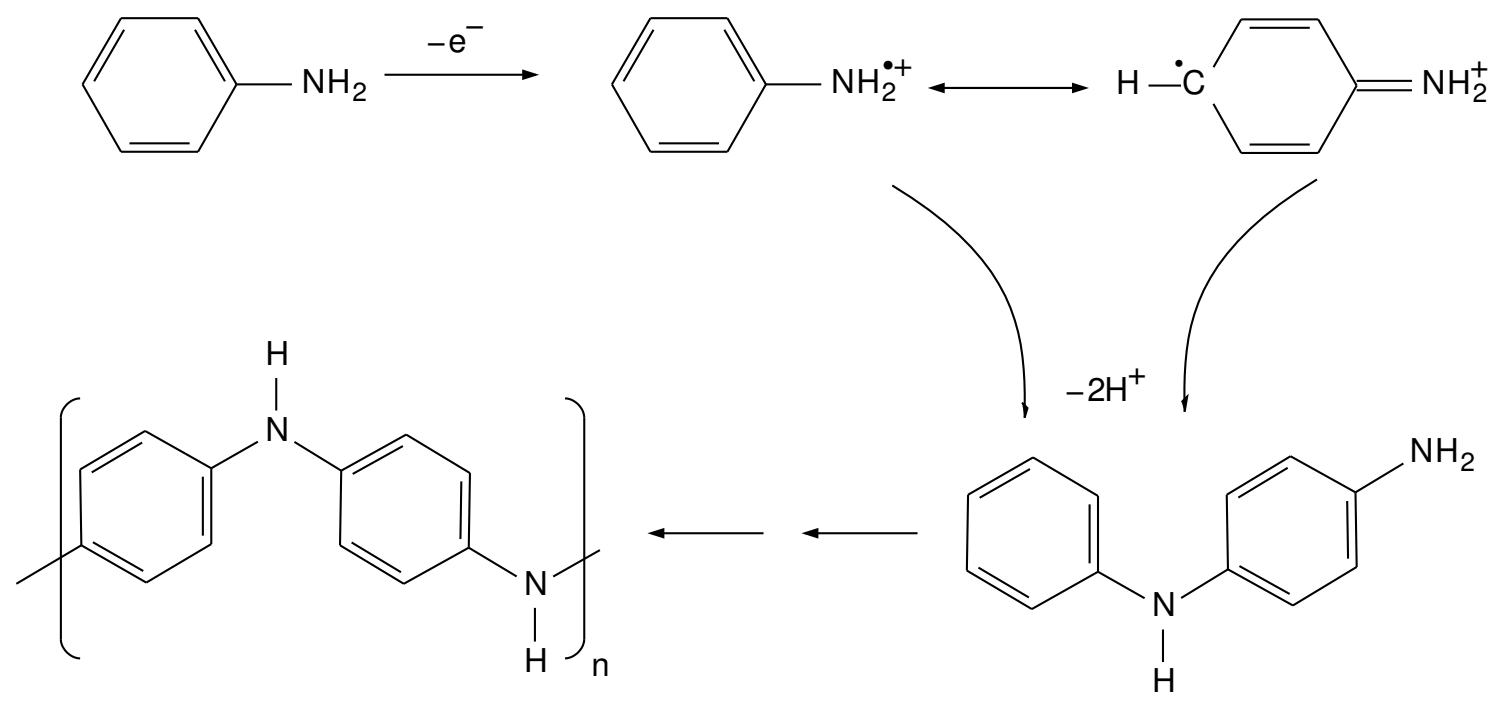

(a)
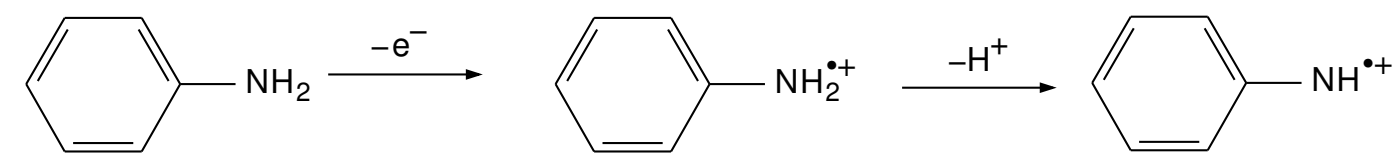<smiles>CN(C)c1ccccc1</smiles><smiles></smiles>

(b)

Figure 6.2 Mechanism for oxidation of aniline: (a) acidic conditions as presented by Wei; ${ }^{169}$ (b) weakly acidic $(\mathrm{pH}>3$ ) or basic conditions as presented by Venancio et al. ${ }^{170}$ Only (a) leads to the formation of polyaniline. 
conditions, so the in situ oxidation method is not amenable to the formation of PANI-NCS composite materials. In cases where the composite material is prepared post-synthesis of the polymer, water-soluble conducting polymer derivatives are often used. For example, Borrmann et al. have reported composites of NCS with the sulfonated PANI derivative PMAS (Figure 6.3). ${ }^{47}$

The requirement for acidic conditions during the polymerisation of polyaniline precludes the in situ oxidation of the monomer on the NCS surface due to the basic nature of the latter. For this reason, the polymer must be prepared prior to its incorporation onto the NCS material. This has been demonstrated before with the NCS-conducting polymer derivatives, using either polypyrrole ${ }^{176}$ and the sulfonated polyaniline derivative PMAS. ${ }^{47}$ PMAS binds to the NCS through coordination between the sulfonate groups of the polymer and $\mathrm{Ca}^{2+}$ ions on the NCS surface. However, this mode of coordination means that the PMAS-NCS composite will dissociate under acidic conditions, as protonation of the sulfonate moieties reduces their effectiveness as ligands for $\mathrm{Ca}^{2+}$. The propensity for the composite material to dissociate reduces its potential effectiveness as an sorbent material for the uptake of pollutant materials such as $\mathrm{ReO}_{4}^{-}$, which are often encountered in acidic solutions.

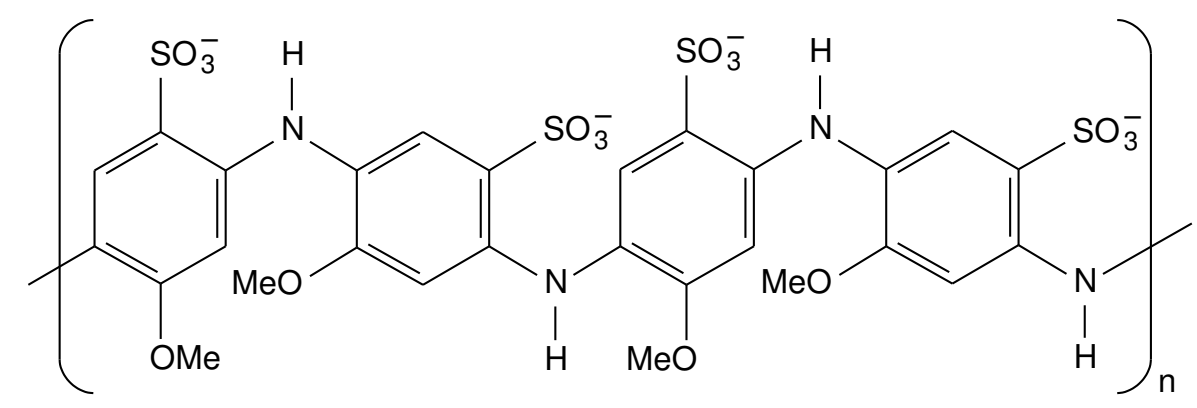

Figure 6.3 Poly(2-methoxyaniline-5-sulfonic acid) (PMAS) in the leucoemeraldine base state. 


\subsubsection{Chemisty of Rhenium}

\subsubsection{Electrochemistry}

Standard reduction potentials for several rhenium (and other group 7) species are listed in Table 6.1, along with those for polyaniline. It can be seen that, under acidic conditions, $\mathrm{ReO}_{4}^{-}$may act to oxidise emeraldine salt to the pernigraniline salt form, with an electrochemical potential for the reaction of $\mathrm{E}^{\ominus}=0.02 \mathrm{~V}$.

Table 6.1 Reduction potentials for various rhenium, manganese, technetium and polyaniline species (after Lide). ${ }^{29}$

\begin{tabular}{lr}
\hline Half Reaction & $\mathbf{E}^{\ominus} / \mathbf{V}$ \\
\hline $\mathrm{PNS}(\mathrm{s})+\mathrm{H}^{+}+\mathrm{e}^{-} \rightleftharpoons \mathrm{ES}(\mathrm{s})^{a}$ & 0.75 \\
$\mathrm{ES}(\mathrm{s})+\mathrm{e}^{-} \rightleftharpoons \mathrm{LEB}(\mathrm{s})$ & 0.30 \\
$\mathrm{ReO}_{4}^{-}+2 \mathrm{H}^{+}+\mathrm{e}^{-} \rightleftharpoons \mathrm{ReO}_{3}(\mathrm{~s})+\mathrm{H}_{2} \mathrm{O}$ & 0.768 \\
$\mathrm{ReO}_{4}^{-}+4 \mathrm{H}^{+}+3 \mathrm{e}^{-} \rightleftharpoons \mathrm{ReO}_{2} \cdot 2 \mathrm{H}_{2} \mathrm{O}(\mathrm{s})$ & 0.51 \\
$\mathrm{ReO}_{4}^{-}+8 \mathrm{H}^{+}+7 \mathrm{e}^{-} \rightleftharpoons \mathrm{Re}(\mathrm{s})+4 \mathrm{H}_{2} \mathrm{O}$ & 0.367 \\
$\mathrm{Re}^{3+}+3 \mathrm{e}^{-} \rightleftharpoons \mathrm{Re}$ & 0.3 \\
$\mathrm{ReO}_{2} \cdot 2 \mathrm{H}_{2} \mathrm{O}+4 \mathrm{H}^{+}+4 \mathrm{e}^{-} \rightleftharpoons \mathrm{Re}(\mathrm{s})+6 \mathrm{H}_{2} \mathrm{O}$ & 0.26 \\
$\mathrm{ReO}_{4}^{-}+4 \mathrm{H}_{2} \mathrm{O}+7 \mathrm{e}^{-} \rightleftharpoons \mathrm{Re}(\mathrm{s})+8 \mathrm{OH}^{-}$ & -0.81 \\
$\mathrm{MnO}_{4}^{-}+8 \mathrm{H}^{+}+5 \mathrm{e}^{-} \rightleftharpoons \mathrm{Mn}^{2+}+2 \mathrm{H}_{2} \mathrm{O}$ & 1.491 \\
$\mathrm{MnO}_{4}^{-}+2 \mathrm{H}_{2} \mathrm{O}+3 \mathrm{e}^{-} \rightleftharpoons \mathrm{MnO}_{2}(\mathrm{~s})+4 \mathrm{OH}^{-}$ & 0.558 \\
$\mathrm{TcO}_{4}^{-}+4 \mathrm{H}^{+}+3 \mathrm{e}^{-} \rightleftharpoons \mathrm{TcO}_{2}(\mathrm{~s})+2 \mathrm{H}_{2} \mathrm{O}$ & 0.738 \\
\hline
\end{tabular}

${ }^{a}$ See Figure 6.1 for the structures of pernigraniline salt, emeraldine salt and leucoemeraldine base.

As this value is only slightly positive, it is prudent to consider how non-standard conditions affect this potential. The concentration dependence of an electrochemical potential may be predicted by the Nernst equation (6.1) with its application to the polyaniline/perrhenate system given in (6.2) and (6.3).$^{\dagger}$ The potential for the reaction as a function of $\mathrm{H}^{+}$and $\mathrm{ReO}_{4}^{-}$concentrations is given in Figure 6.4. It can be seen in the figure that a positive electrochemical potential only occurs when both species are present at relatively high (near-molar) concentrations. As will be shown in Section 6.2, sorption of $\mathrm{ReO}_{4}^{-}$may lower the rhenium concentration to sub-millimolar levels. As such, it is

\footnotetext{
${ }^{\dagger}$ Although the redox-change for polyaniline is shown as an oxidation of emeraldine salt to pernigraniline salt, the same equation is valid for the oxidation of emeraldine base to pernigraniline base.
} 
highly unlikely that redox chemistry contributes in a significant manner to the sorption process of $\mathrm{ReO}_{4}^{-}$by as-made emeraldine polyaniline species.

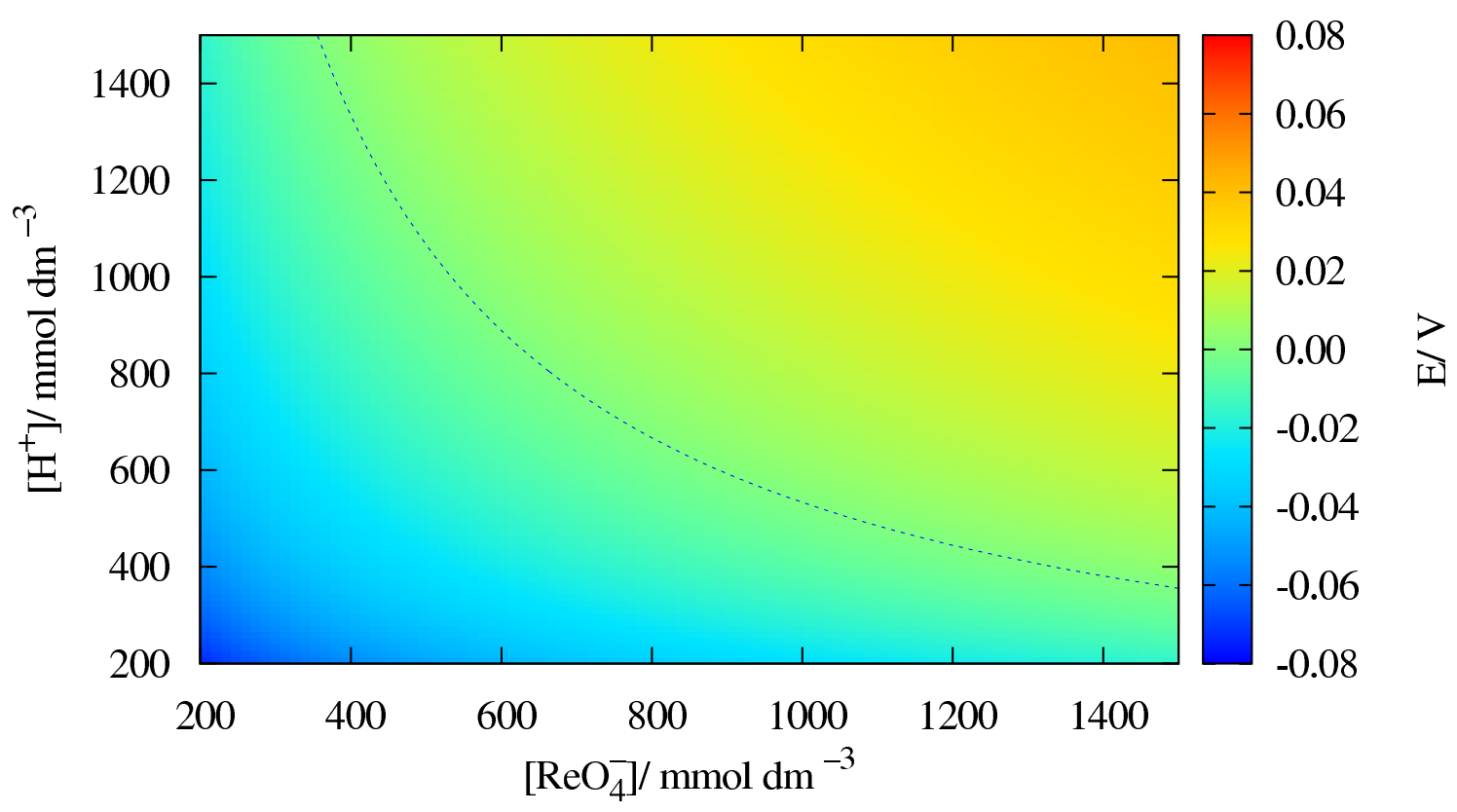

Figure 6.4 Electrochemical potential for the reduction of $\mathrm{ReO}_{4}^{-}$by polyaniline in the emeraldine salt oxidation state at $25^{\circ} \mathrm{C}$, as predicted by the Nernst equation (6.2). The $0 \mathrm{~V}$ potential contour is indicated in the figure.

$$
\begin{gathered}
\mathrm{E}=\mathrm{E}^{\ominus}-\frac{R T}{n F} \ln Q \\
\mathrm{ES}+\mathrm{ReO}_{4}^{-}+\mathrm{H}^{+} \rightleftharpoons \mathrm{PNS}+\mathrm{ReO}_{3}(\mathrm{~s})+\mathrm{H}_{2} \mathrm{O} \\
\mathrm{E}=\mathrm{E}^{\ominus}-\frac{R T}{F} \ln \left(\frac{1}{\left[\mathrm{ReO}_{4}^{-}\right]\left[\mathrm{H}^{+}\right]}\right)
\end{gathered}
$$

\subsubsection{Coordination Chemistry}

Several compounds containing rhenium in the +7 oxidation state have been reported in which the rhenium is coordinated to nitrogen atoms, for example the 1,4,7-triazacyclononane and the bis(trimethylsilyl)-benzamidinate complexes shown in Figure 6.5. Complexes such as these are normally formed from $\operatorname{Re}_{2} \mathrm{O}_{7}$. During the formation of the complexes, the $\mathrm{Re}_{2} \mathrm{O}_{7}$ will decompose to the $\mathrm{ReO}_{4}^{-}$anion and a $\mathrm{ReO}_{3}^{+}$moiety:

$$
\mathrm{Re}_{2} \mathrm{O}_{7} \rightleftharpoons \mathrm{ReO}_{3}^{+}+\mathrm{ReO}_{4}^{-}
$$


Complexes such as those in Figure 6.5 are not usually formed directly from the $\mathrm{ReO}_{4}^{-}$ anion. However, complexes such as $\mathrm{Gd}\left(\mathrm{ReO}_{4}\right)_{3}\left(\mathrm{H}_{2} \mathrm{O}\right)_{3},{ }^{178}$ in which $\mathrm{ReO}_{4}^{-}$acts as a ligand are also known. The position of the $\mathrm{ReO}_{4}^{-}$anion in the spectrochemical series has been reported to lie between $\mathrm{ClO}_{4}^{-}$(on the lower side) and $\mathrm{CH}_{3} \mathrm{COO}^{-}$(on the upper side). ${ }^{179}$ This means that the anion is modestly amenable to coordination and will replace $\mathrm{ClO}_{4}^{-}$ ligands coordinated to metal ions.

\subsubsection{Rhenium Sorption}

In oxic media such as water, rhenium and technetium exist primarily as oxyanions in the +7 oxidation state (perrhenate, $\mathrm{ReO}_{4}^{-}$, and pertechnetate, $\mathrm{TcO}_{4}^{-}$). Under acidic conditions, the ions act as moderate oxidising agents, forming $\mathrm{ReO}_{3}(\mathrm{~s})$ and $\mathrm{TcO}_{2}(\mathrm{~s})$ with standard reduction potentials of $\mathrm{E}^{\ominus} \mathrm{ReO}_{4}^{-} / \mathrm{ReO}_{3}=0.768 \mathrm{~V}$ and $\mathrm{E}^{\ominus} \mathrm{TcO}_{4}^{-} / \mathrm{TcO}_{2}=0.738 \mathrm{~V}$ respectively. A more complete listing of reduction potentials for rhenium and oxides and oxyanions is given in Table 6.1. In addition to its use as a model for technetium, rhenium compounds are valuable in their own right, often being used as catalysts in, for example, alkene metathesis ${ }^{180,181}$ or as potential catalysts for the photo-induced reduction of $\mathrm{CO}_{2} \cdot{ }^{182}$

In Nature, rhenium compounds are commonly found as minor components of molybdate and copper ores. ${ }^{183,184}$ Perrhenate is typically recovered from these ores through its sorption by amine-based anion exchangers, ${ }^{184,185}$ before its recovery as a perrhenate salt such as ammonium perrhenate, $\mathrm{NH}_{4} \mathrm{ReO}_{4}$. This final step is often performed by evaporation of an $\mathrm{NH}_{4} \mathrm{ReO}_{4}$ solution, although other methods of rhenium recovery are known, for

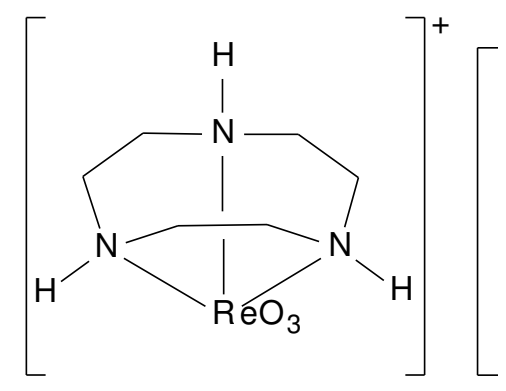

(a)

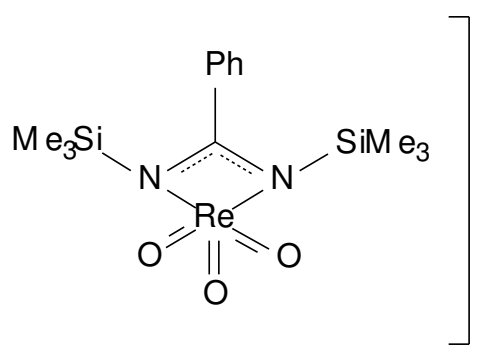

(b)

Figure 6.5 Examples of rhenium(VII) complexes in exhibiting $\mathrm{Re}-\mathrm{N}$ bonding: (a) complex with 1,4,7-triazacyclononane; (b) complex with bis(trimethylsilyl)-benzamidinate, as reported by Abram. ${ }^{177}$ 
example electroreduction ${ }^{186}$ or recovery as other salts, such as $\left[\mathrm{Zn}\left(\mathrm{NH}_{3}\right)_{4}\right]\left(\mathrm{ReO}_{4}\right)_{2} \cdot{ }^{187}$ Perrhenate, as an anion, is not readily sorbed by negatively charged surfaces, such as silicates, ${ }^{188}$ and calcium perrhenates typically exhibit a high degree of solubility (187 $\mathrm{g}$ $\mathrm{Ca}\left(\mathrm{ReO}_{4}\right)_{2}$ will dissolve in $100 \mathrm{~g}$ water at $\left.30^{\circ} \mathrm{C}\right) .{ }^{189}$ For this reason, a material such as NCS would, on its own, make a poor sorbent for $\mathrm{ReO}_{4}^{-}$. Most reports of rhenium recovery techniques rely on ion exchange ${ }^{184,185,190,191}$ or electrodeposition. ${ }^{192,193}$

Zemskova et al. ${ }^{194}$ have reported the use of chitosan-functionalised carbon fibre sheets as sorbent materials for $\mathrm{ReO}_{4}^{-}$, with the sorbent exhibiting Langmuir isotherm behaviour. The authors concluded that the electrostatic interactions between the amino groups and $\mathrm{ReO}_{4}^{-}$was responsible for the sorption of the latter. By applying a negative potential to the sorbent material, it was possible to reversibly desorb the $\mathrm{ReO}_{4}^{-}$. Sorbent materials exhibiting Freundlich isotherm behaviour for $\mathrm{ReO}_{4}^{-}$sorption are also known, for example the polymer resins reported by Xiong et al. ${ }^{195}$ (Figure 6.6).

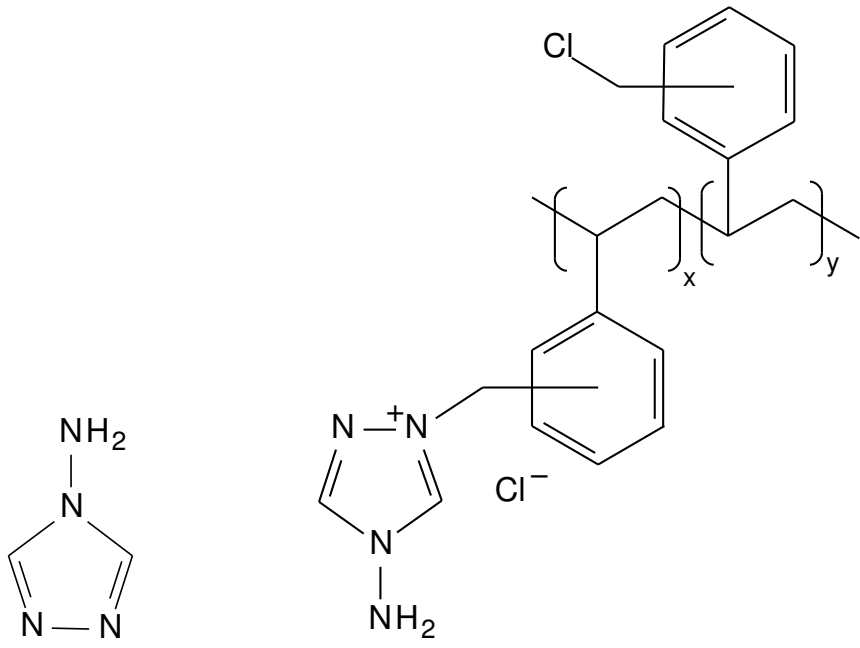

(a)

(b)

Figure 6.6 Polymer resin used by Xiong et al. ${ }^{195}$ for $\mathrm{ReO}_{4}^{-}$sorption: (a) 4-amino-1,2,4-triazole; (b) functionalised chloromethylated polystyrene.

The use of chitosan as an sorbent for $\mathrm{ReO}_{4}^{-}$in its own right has been reported by Kim et al. ${ }^{188}$ The sorption was found to be highly dependant on the solution $\mathrm{pH}$ and ionic strength, with the greatest sorption occurring at low $\mathrm{pH}$ values and low ionic strength. The authors attributed this sorption to an electrostatic interaction between the $\mathrm{ReO}_{4}^{-}$ and the $\mathrm{R}-\mathrm{NH}_{3}^{+}$functional groups of the chitosan. ${ }^{\dagger}$ The higher $\mathrm{ReO}_{4}^{-}$sorption at low

$\dagger$ It should be noted that in the $\mathrm{pH}$ range studied by Kim et al. ${ }^{188}$ (3-7), perrhenate remains unprotonated, the $\mathrm{p} K_{a}$ of the acid being -1.25 . ${ }^{196,197}$ 
ionic strength was attributed to the presence of two different interactions occurring with the amine groups. Electrostatic interactions can occur between chitosan and $\mathrm{ReO}_{4}^{-}$, or between the chitosan and the ionic-strength determinant (in this case $\mathrm{NO}_{3}^{-}$). Stability constants for these ion pairs are given in Table 6.2. As the equilibrium constant for the

Table 6.2 Equilibria determining rhenium uptake by chitosan (after Kim ${ }^{188}$ ).

\begin{tabular}{lr}
\hline Equilibrium & $\log K$ \\
\hline $\mathrm{R}-\mathrm{NH}_{3}^{+}+\mathrm{ReO}_{4}^{-} \rightleftharpoons \mathrm{R}-\mathrm{NH}_{3}^{+} \mathrm{ReO}_{4}^{-}$ & 1.24 \\
$\mathrm{R}-\mathrm{NH}_{3}^{+}+\mathrm{NO}_{3}^{-} \rightleftharpoons \mathrm{R}-\mathrm{NH}_{3}^{+} \mathrm{NO}_{3}^{-}$ & 1.55 \\
$\mathrm{R}-\mathrm{NH}_{2}+\mathrm{H}^{+} \rightleftharpoons \mathrm{R}-\mathrm{NH}_{3}^{+}$ & 5.8 \\
\hline
\end{tabular}

formation of the chitosan-nitrate ion pair is slightly larger than that for the formation of the chitosan-perrhenate ion pair, it can be seen that the presence of nitrate can significantly retard the sorption of perrhenate.

Petrov et al. ${ }^{198}$ have reported the presence of hydrogen bonding between $\mathrm{ReO}_{4}^{-}$and various amine species (Figure 6.7). They report that the strength of an individual hydrogen bond increases in the order $1^{\circ}<2^{\circ}<3^{\circ}$ amines and attributed this to the greater basicity of the more substituted amines. Counteracting this bonding is the possibility of $\mathrm{ReO}_{4}^{-}$ to form multiple hydrogen bonds to a single amine functional group (Figure 6.7(b)). As such, an amine with a higher degree of substitution will form fewer, but stronger, hydrogen bonds to an individual $\mathrm{ReO}_{4}^{-}$ion. Thus, the total strength of the interactions between the two components $\left(\mathrm{ReO}_{4}^{-}\right.$and the amine) is not necessarily greater for the higher degree amines.

Kholmogorov et al. ${ }^{185}$ have reported the use of several amine-based ion exchangers as selective sorbents for $\mathrm{ReO}_{4}^{-}$from a mixed perrhenate/molybdate solution. The greatest distribution coefficients for $\mathrm{ReO}_{4}^{-}$were reported for ion exchangers containing the amine functional groups shown in Figure 6.8. Distribution coefficients in excess of 1200 were reported for all these resins. The ion exchangers, amine functionalised poly(styrene/divinylbenzenes), performed significantly better when prepared as a gel instead of using a templating agent (isooctane) to provide porosity within the structure. By comparison, the porous ion exchanges compared poorly, having a distribution coefficient with respect to $\mathrm{ReO}_{4}^{-}$of 200-300, approximately one quarter that of the gel-based 
exchangers. The use of aniline based ion exchangers was also investigated. A distribution coefficient of 236 was reported for a porous aniline based ion exchanger; no ion-exchange results were reported for an aniline-based gel.

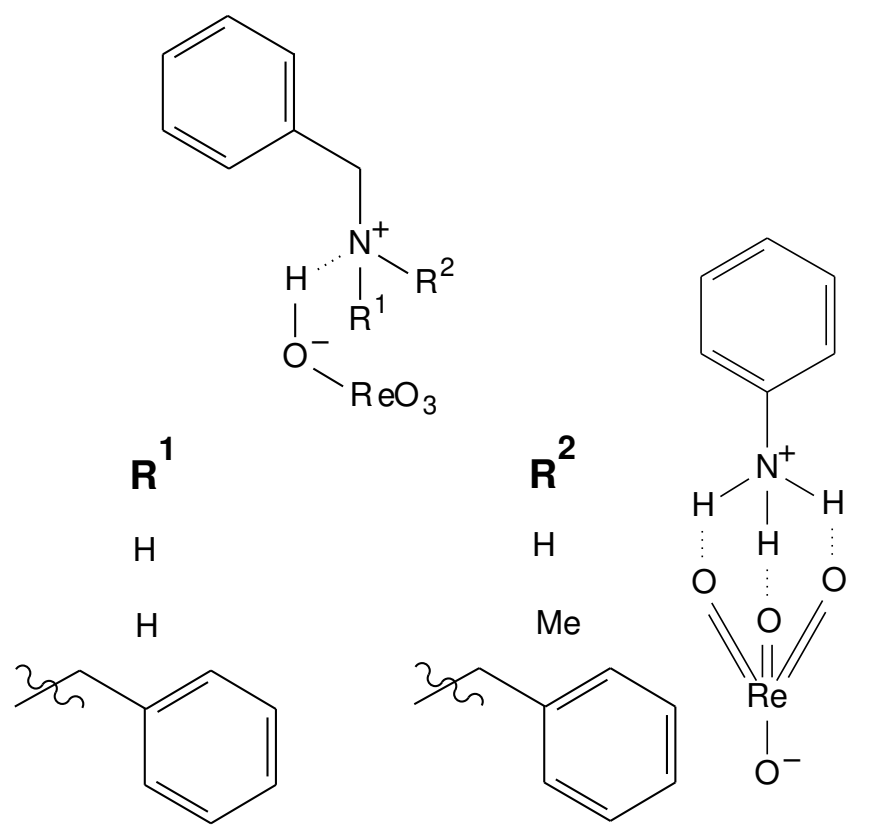

(a)

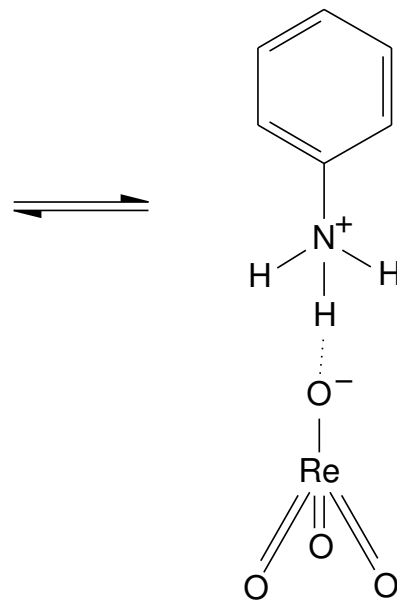

(b)

Figure 6.7 Examples of hydrogen bonding between $\mathrm{ReO}_{4}^{-}$and amine species ((a)), some of which may form multiple hydrogen bonds to the same pehrrhenate ion, (b) (after Petrov et al. ${ }^{198}$ ).

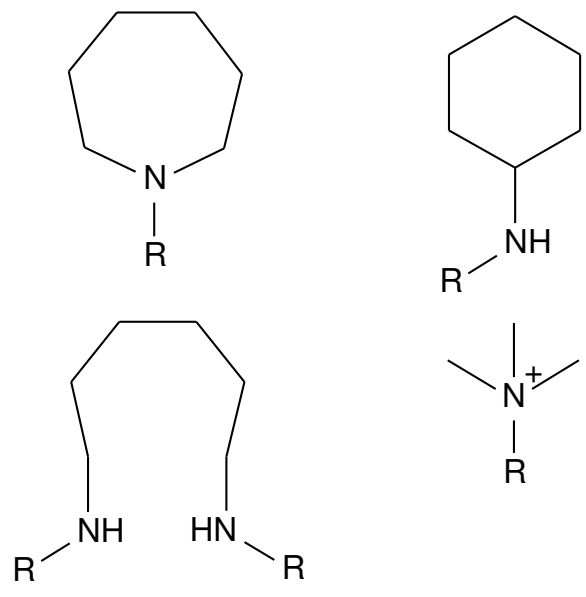

Figure 6.8 Amine functional groups reported as suitable for ion exchange of $\mathrm{ReO}_{4}^{-}$by Kholmogorov et al. ${ }^{185}$ 


\subsection{Results}

Characterisation of the PANI-NCS composites were undertaken via a variety of instrumental techniques. The applicability of the composite material towards $\mathrm{ReO}_{4}^{-}$sorption was investigated.

\subsubsection{Polyaniline-NCS Composites}

\subsubsection{UV-Visible Spectroscopy}

Figures 6.9-6.10 show the reflectance spectra for a the polyaniline-NCS composite materials. Included in Figure 6.9 is an absorbance UV/vis spectrum for a polyaniline film in the emeraldine base oxidation state. The peak positions of these spectra are given in Table 6.3. In emeraldine base, the two peaks observed in the UV/vis spectrum may be attributed to a $\pi \rightarrow \pi^{*}$ transition at $\lambda_{\max } \approx 320 \mathrm{~nm}$ and a broad $\mathrm{n} \rightarrow \pi^{*}$ transition at $\lambda_{\max } \approx 600 \mathrm{~nm} .{ }^{168}$ Density functional theory (DFT) calculations have shown that the $\mathrm{n}$ orbital resides on the amine nitrogen atoms in polyaniline. ${ }^{167}$ By comparing the peak positions in the reflectance spectra of a PANI-NCS composite with those of the emeraldine base absorption spectrum, it can be seen that the $\mathrm{n} \rightarrow \pi^{*}$ transition has reduced in energy; centred at a wavelength of $\lambda_{\max } \approx 630 \mathrm{~nm}$ compared to $\lambda_{\max }=575 \mathrm{~nm}$ in the reference spectrum. The energy of the $\pi \rightarrow \pi^{*}$ transition remains largely unchanged meaning that the $\mathrm{n}$ orbital has increased in energy rather than the $\pi^{*}$ orbital decreasing in energy..$^{\dagger}$ The energy difference between the $\mathrm{n} \rightarrow \pi^{*}$ transitions in the unmodified PANI and the composite (Equation (6.5)) is approximately $18 \mathrm{~kJ} \mathrm{~mol}^{-1}$. The magnitude of this energy difference suggests that the PANI is bound to the NCS material through hydrogen bonding, rather than covalent bonding. As the $\mathrm{n}$ orbital has increased in energy, the hydrogen bonding is most likely occurring between the amine hydrogen and an NCS oxygen, rather than the amine nitrogen and an NCS hydrogen. The alternative is hydrogen bonding between a hydroxyl group and an imine nitrogen, as protonation of the imine nitrogen, as occurs with emeraldine salt is known to induce a red-shift in the $\mathrm{n} \rightarrow \pi^{*}$

${ }^{\dagger}$ DFT calculations suggest that the $\pi^{*}$ orbitals for the $\mathrm{n} \rightarrow \pi^{*}$ and $\pi \rightarrow \pi^{*}$ transitions are degenerate. ${ }^{167}$ 
transition. ${ }^{168}$ Examples of these hydrogen bonding modes are given in Figure 6.11.
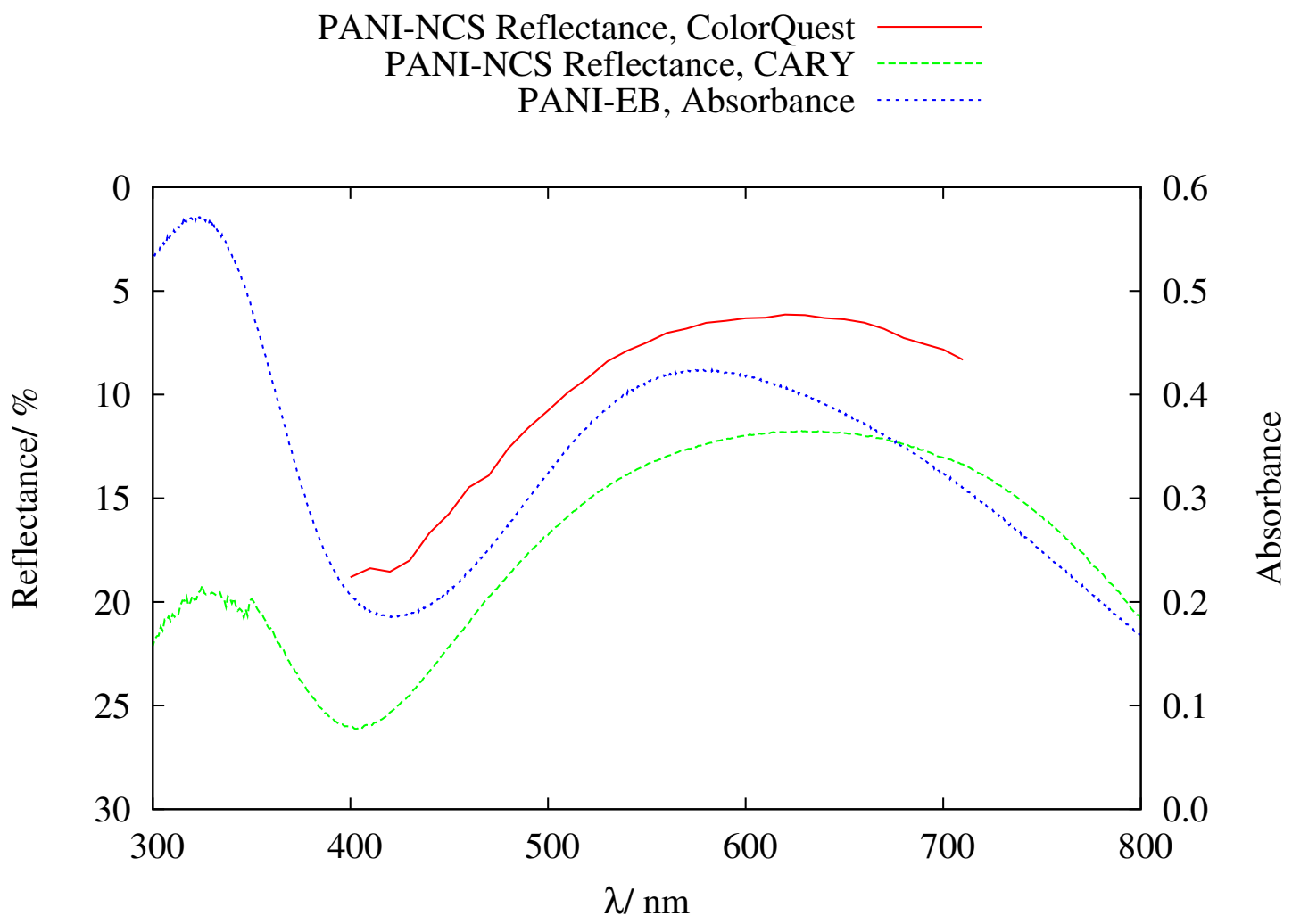

Figure 6.9 UV/visible reflectance spectra of PANI-NCS composite material, with absorption spectrum of polyaniline, as emeraldine base, for comparison purposes.

NCS PANI-NCS

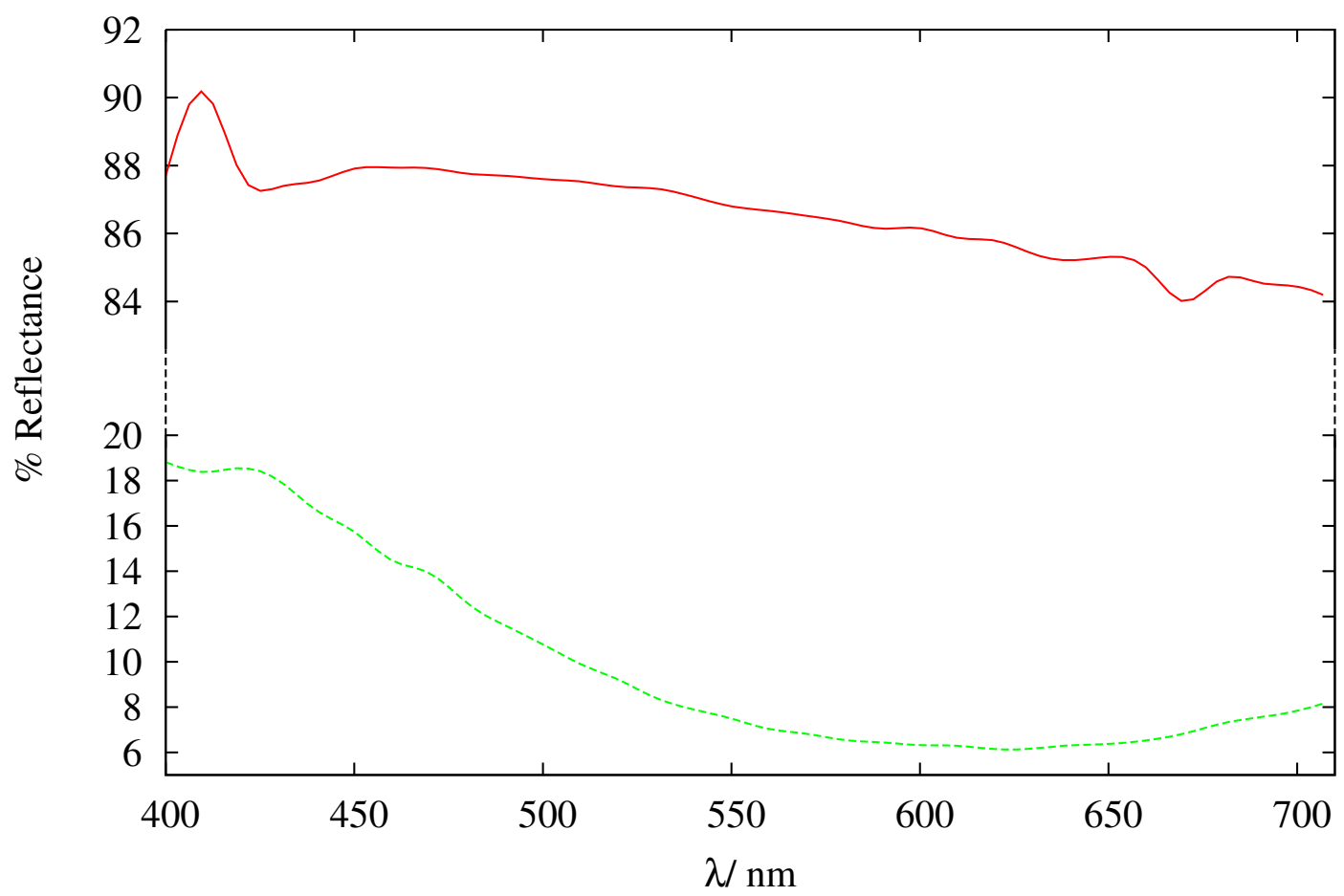

Figure 6.10 Reflectance spectra of NCS and its PANI composite. 


$$
\Delta E=h c\left(\frac{1}{\lambda_{1}}-\frac{1}{\lambda_{2}}\right)
$$

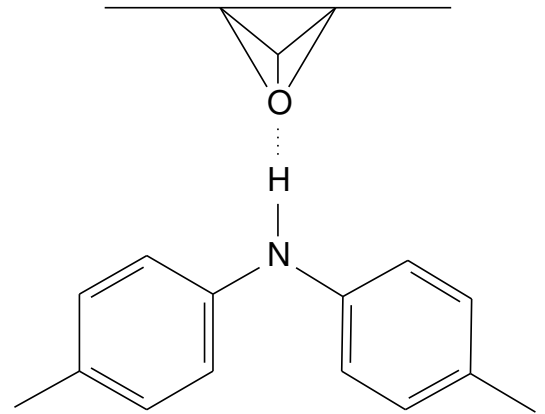

(a)<smiles>CC1OC(C)C1C</smiles><smiles>Cc1ccc(Nc2ccc(C)cc2)cc1</smiles>

(b)

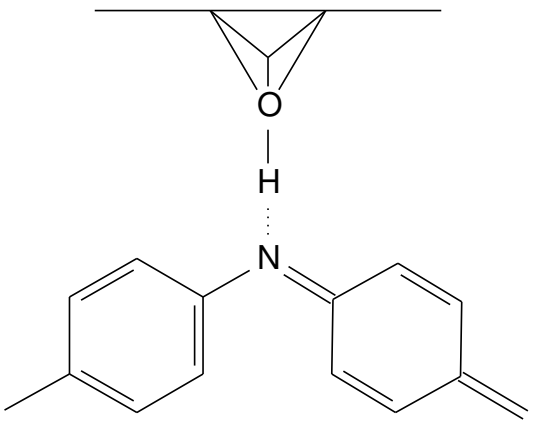

(c)

Figure 6.11 Possible hydrogen bonding modes between emeraldine base and NCS. Bonding modes (a) and (c) are most likely as they lead to a red shift in $\lambda_{\max }$ absorption for the $\mathrm{n} \rightarrow \pi^{*}$ transition.

Table 6.3 Spectral parameters for PANI-NCS composite, from spectra presented in Figure 6.9.

\begin{tabular}{lrr}
\hline Instrument & $\lambda_{\max } / \mathbf{n m}$ & \% Reflectance \\
\hline HunterLabs ColorQuest C5160 & $620-630^{a}$ & $6.1-6.2$ \\
Varian Cary Scan 100 Reflectance & 325 & 19.22 \\
& 628 & 11.75 \\
Varian Cary Scan 100 UV & 322 & N/A \\
& 575 & N/A \\
\hline
\end{tabular}

${ }^{a}$ The HunterLabs instrument has a spectral resolution of $10 \mathrm{~nm}$, compared to $2 \mathrm{~nm}$ for the Varian instrument.

${ }^{b} \mathrm{UV} / \mathrm{vis}$ absorbance spectrum of PANI in the emeraldine base oxidation state. PANI film deposited on walls of disposable cuvette, spectrum recorded in $0.1 \mathrm{~mol} \mathrm{dm}^{-3}$ $\mathrm{NaOH}$ on the same Varian instrument as was used for the reflectance measurement (Figure 6.13). 
The effect of $\mathrm{ReO}_{4}^{-}$sorption on the spectrographic properties of polyaniline films is shown in Figure 6.12, with Figure 6.13 provided for comparison purposes showing the UV/vis spectrographic properties of the various oxidation states of polyaniline. Labels in Figure 6.12 indicate the initial state of the polymer prior to its exposure to $\mathrm{ReO}_{4}^{-}$. It can be seen in Figure 6.12 that $\mathrm{ReO}_{4}^{-}$, under acidic conditions, does not significantly alter the spectrographic properties of the emeraldine salt form of polyaniline. As the spectrographic properties of polyaniline are highly dependant on the redox state of the polymer, this indicates that no significant redox reaction occurs between an emeraldine salt film and $\mathrm{ReO}_{4}^{-}$under acidic conditions. The small changes observed in the UV/vis spectrum of emeraldine salt in contact with $\mathrm{ReO}_{4}^{-}$do not preclude the existence of an electrostatic interaction between $\mathrm{ReO}_{4}^{-}$and ES. However, it is unlikely that hydrogen bonding is occurring between these species. By comparison, it can be seen that the reaction of $\mathrm{ReO}_{4}^{-}$with the leucoemeraldine base form of polyaniline causes significant changes in the spectrographic properties of the polymer. The characteristic absorption peak of LEB, at $\lambda_{\max }=330 \mathrm{~nm}$ decreases in intensity, and undergoes a red shift, approaching the peak of ES located at $\lambda_{\max }=380 \mathrm{~nm}$. An additional peak also starts to appear at $\lambda_{\max } \geq 800 \mathrm{~nm}$, also characteristic of the emeraldine salt oxidation state of polyaniline. This indicates that, under acidic conditions, $\mathrm{ReO}_{4}^{-}$will readily oxidise leucoemeraldine base to emeraldine salt. For this to occur, the $\mathrm{ReO}_{4}^{-}$must undergo reduction, the thermodynamically favoured product of which is $\mathrm{ReO}_{3}$. 


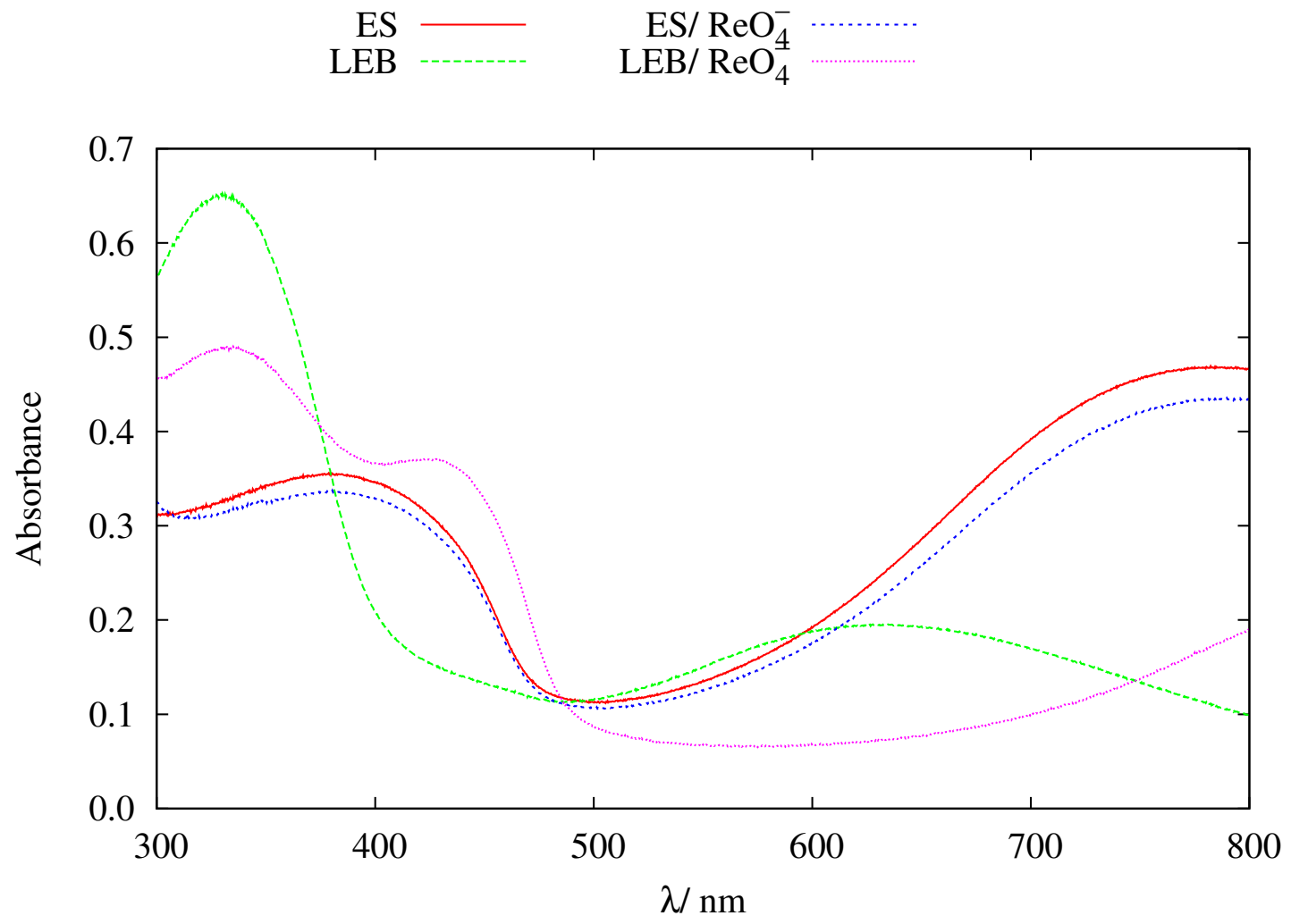

Figure 6.12 UV/vis spectra of perrhenate sorption onto polyaniline films deposited onto polycarbonate cuvettes. The polyaniline was treated as per Figure 6.13 , then rinsed with water and a solution containing $0.1 \mathrm{~mol} \mathrm{dm}{ }^{-3} \mathrm{HCl}$ and $5.4 \mathrm{mmol} \mathrm{dm}^{-3} \mathrm{KReO}_{4}$ introduced to the cuvette. 5 minutes were allowed for equilibration before the spectra were recorded. Labels in the figure indicate the initial oxidation state of the polymer prior to this equilibration time. Thus, the trace labelled ' $\mathrm{LEB} / \mathrm{ReO}_{4}^{-}$' shows partial oxidation of the leucoemeraldine base to the emeraldine salt form of polyaniline. 


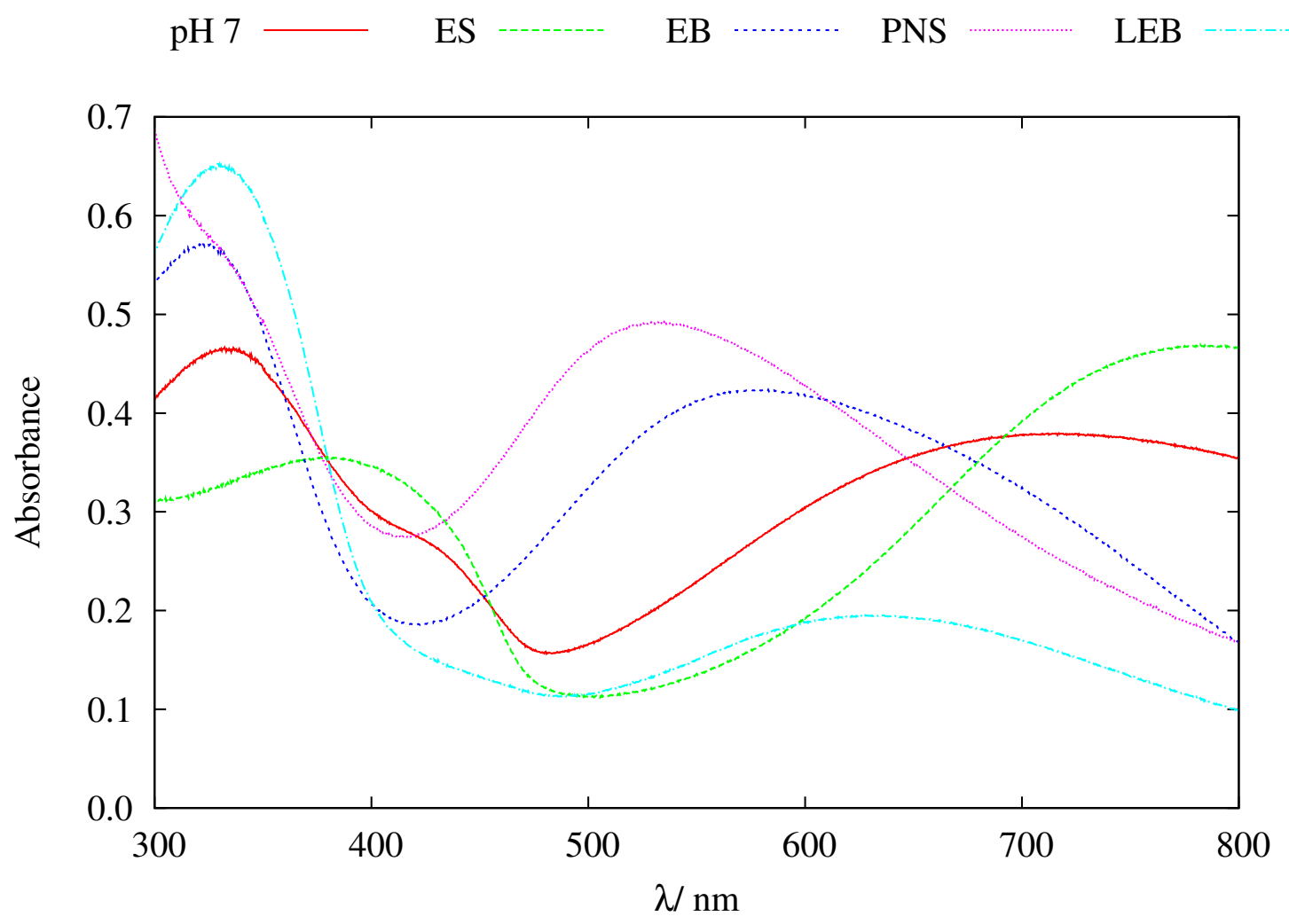

Figure 6.13 UV/vis spectra of polyaniline films deposited on polycarbonate cuvettes. Spectra were recorded in: water (pH 7) $0.1 \mathrm{~mol} \mathrm{dm}^{-3} \mathrm{HCl}$ (ES); $0.1 \mathrm{~mol} \mathrm{dm}^{-3} \mathrm{NaOH}$ (EB); $0.1 \mathrm{~mol} \mathrm{dm}^{-3}\left(\mathrm{NH}_{4}\right)_{2} \mathrm{~S}_{2} \mathrm{O}_{8}$ (PNS); $0.1 \mathrm{~mol} \mathrm{dm}{ }^{-3} \mathrm{~N}_{2} \mathrm{H}_{2} \cdot \mathrm{H}_{2} \mathrm{O}$ (LEB) 


\subsubsection{Thermogravimetric Analysis}

A typical thermogravimetric trace of the PANI-NCS materials is presented in Figure 6.14. The trace shows a $15.6 \%$ mass loss between $105^{\circ} \mathrm{C}$ and $950^{\circ} \mathrm{C}$ for a composite material containing nominally $10 \mathrm{wt} \%$ PANI. This is slightly higher than would be expected. However, the NCS material does contain a significant quantity of water present in the material, both as surface coordinated water and as hydroxyl functionalities, the latter of which will dehydrate at approximately $650-680^{\circ} \mathrm{C}$, following:

$$
(\mathrm{CaO})_{0.8} \mathrm{SiO}_{(2-x)}(\mathrm{OH})_{2 x} \longrightarrow(\mathrm{CaO})_{0.8} \mathrm{SiO}_{2}+\mathrm{H}_{2} \mathrm{O} \uparrow
$$

Between $105^{\circ} \mathrm{C}$ and $600^{\circ} \mathrm{C}$, the material loses $12.9 \%$ of its mass, the majority of which could be attributed to the combustion of polyaniline.

Mass $\longrightarrow$ Temperature

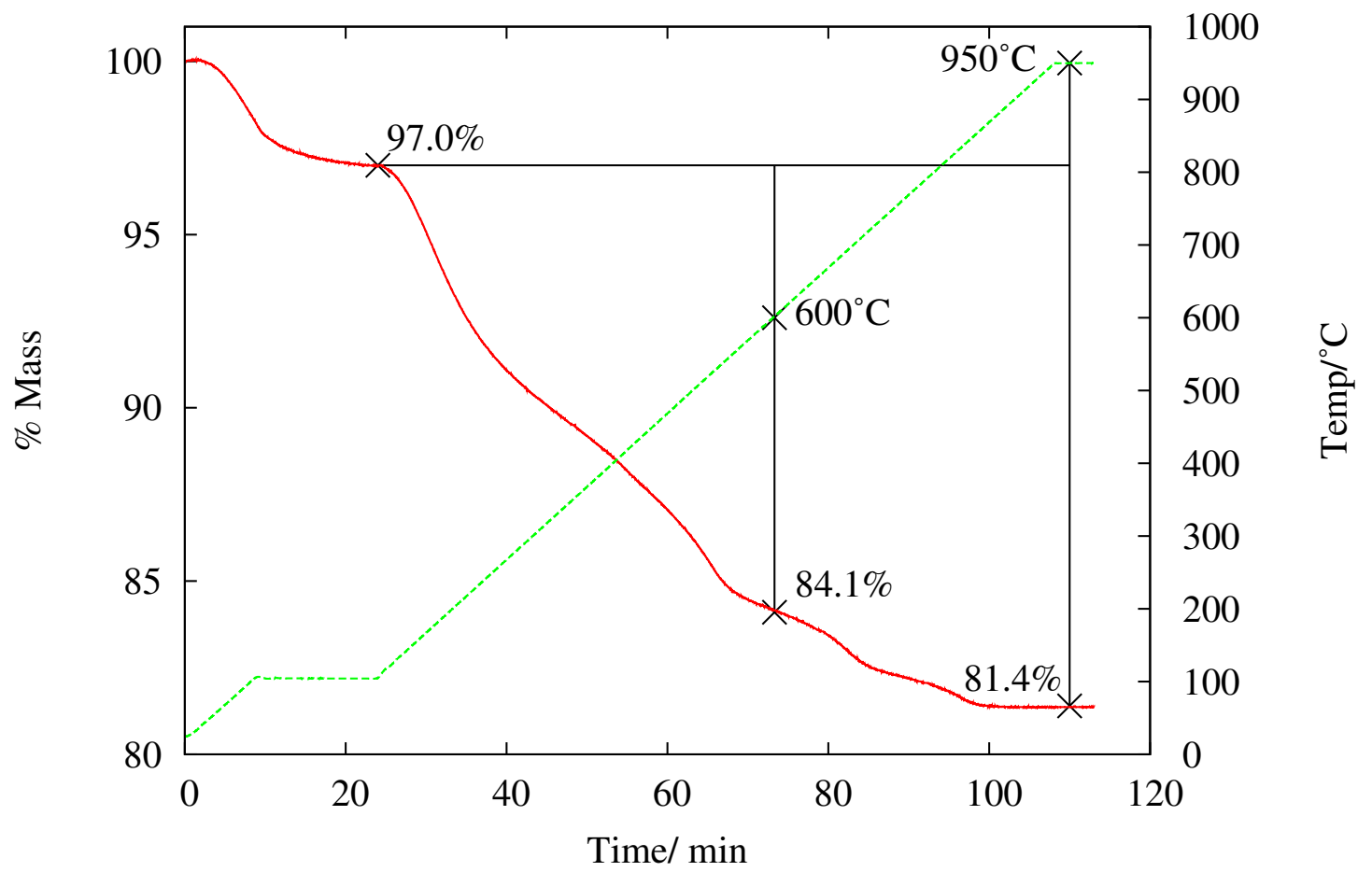

Figure 6.14 TGA trace for PANI-NCS composite with a nominal $10 \mathrm{wt} \%$ PANI content. 


\subsubsection{Electrochemistry}

Figure 6.15 shows a cyclic voltagram of a PANI-NCS composite recorded in a saturated $\mathrm{KClO}_{4}$ solution at $\mathrm{pH}$ 7. The material is largely redox inactive, with the exception of a small reduction peak occurring between -0.10 and $-0.20 \mathrm{~V}$, relative to the standard hydrogen electrode. This is most likely due to the reduction of emeraldine base to leucoemeraldine base, which is consistent with the same transition in unmodified polyaniline at $\mathrm{pH} 7$ (Equation (6.1)). The corresponding oxidation peak (from leucoemeraldine base to emeraldine base) is ill defined, and the transition between the emeraldine and pernigraniline forms is not observed.

As the presence of polyaniline in the PANI-NCS composites has been confirmed spectrographically, the non-observation in cyclic voltammetry of a emeraldine - pernigraniline redox transition does not indicate a lack of the transition itself. Most likely, a poor electrical connectivity between the PANI-NCS powder and the working electrode of the cyclic voltammeter prevented the transition from being observed.

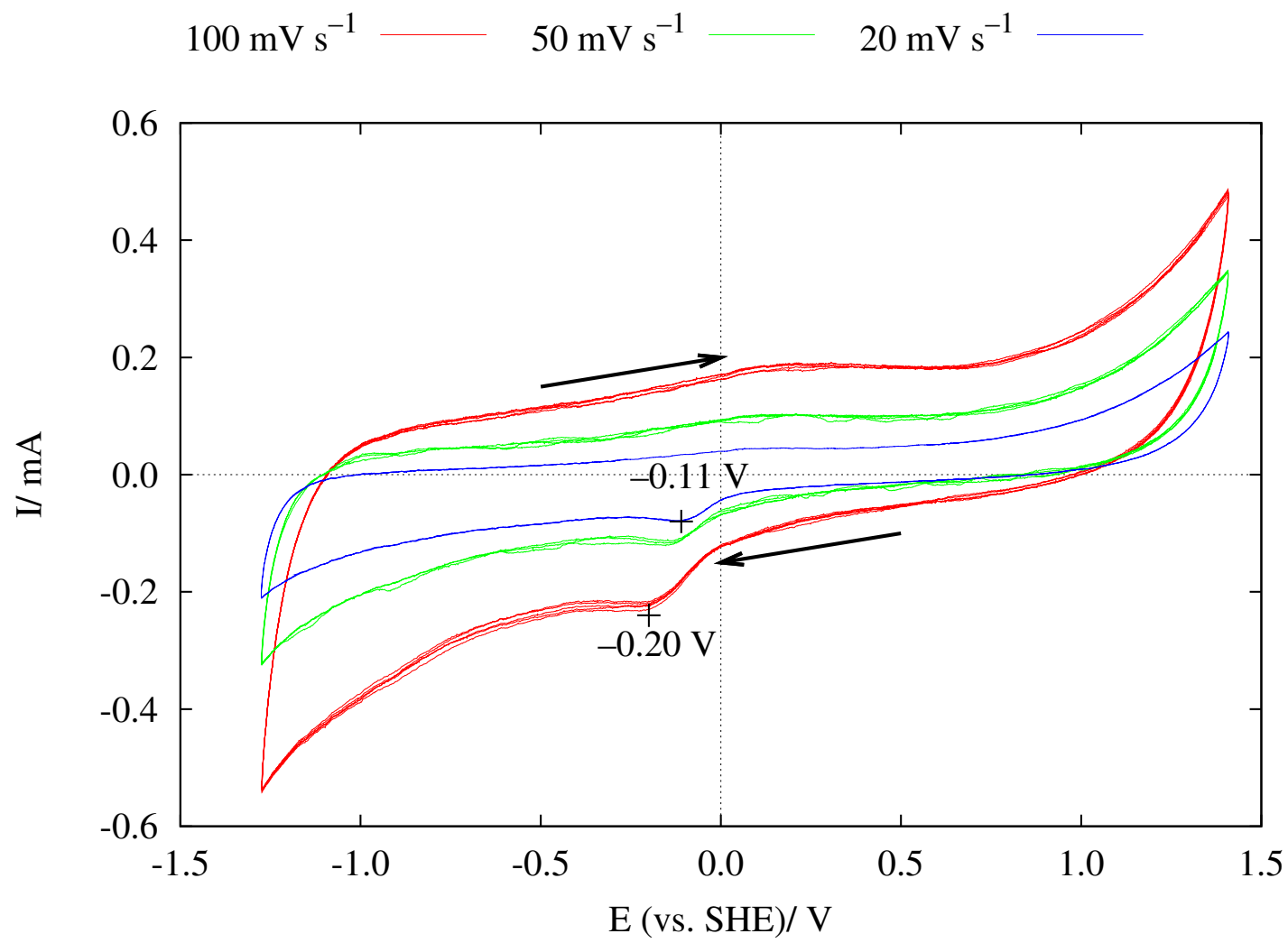

Figure 6.15 Cyclic voltagram of PANI-NCS composite material. Voltagram recorded in saturated $\mathrm{KClO}_{4}$ at various sweep rates. Potentials are referenced to a standard hydrogen electrode. 
The Pourbaix diagram ${ }^{199}$ for the rhenium system is given in Figure 6.16. The diagram, which shows the $\mathrm{pH}$ dependence of the reduction potential for the equilibrium between various rhenium oxidation states, indicates that for the $\mathrm{pH}$ range observed for the rhenium sorption by the PANI-NCS composite, $\mathrm{ReO}_{4}^{-}$may exist in equilibrium with any of the rhenium oxides $\mathrm{ReO}_{3}, \mathrm{ReO}_{2}$ or $\mathrm{Re}_{2} \mathrm{O}_{3}$. The energy of the $\mathrm{Re} 4 \mathrm{f}$ XPS signal for the rhenium sorbed onto the PANI-NCS occurs midway between that of the $\mathrm{ReO}_{2}$ and $\mathrm{Re}$.

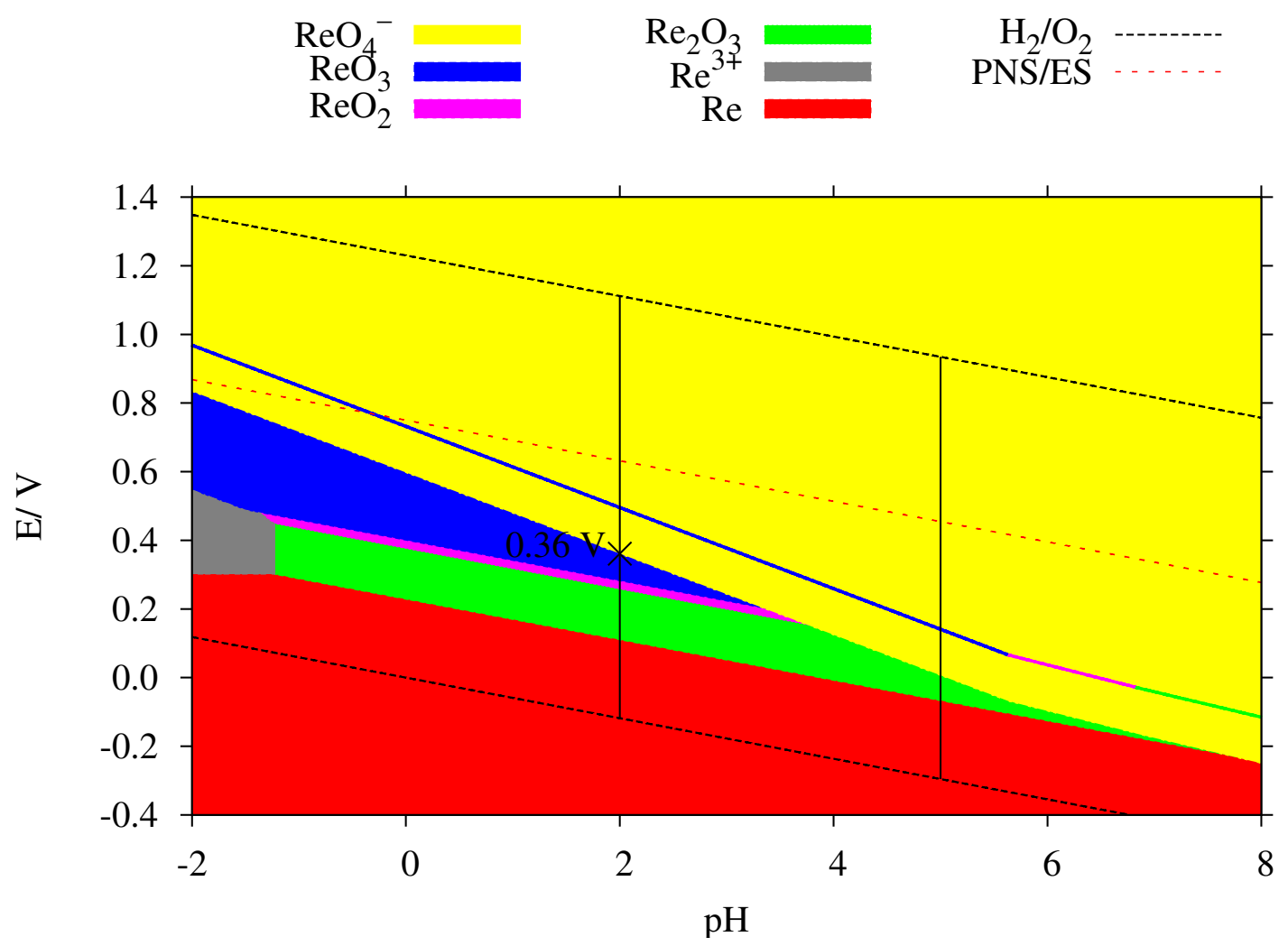

Figure 6.16 Partial Pourbaix diagram for the rhenium system. Potentials in the diagram calculated for $\left[\mathrm{ReO}_{4}^{-}\right]=5.4 \mathrm{mmol} \mathrm{dm}{ }^{-3}$, the maximum initial $\mathrm{ReO}_{4}^{-}$concentration for the PANI-NCS sorption study. The potential calculation for $\left[\mathrm{ReO}_{4}^{-}\right]=1 \mathrm{~mol} \mathrm{dm}^{-3}$ is given by the multicoloured line, and the potential for the $\mathrm{PNS}+\mathrm{H}^{+}+\mathrm{e}^{-} \rightleftharpoons \mathrm{ES}$ reaction is shown by the broken red line. Vertical lines indicate the $\mathrm{pH}$ range observed for the uptake of $\mathrm{ReO}_{4}^{-}$by PANI-NCS composites (see Figure 6.23). Diagram constructed from equations presented by Zoubov and Pourbaix. ${ }^{199}$ Oxidation states of rhenium not observed in aqueous systems, for example $\mathrm{Re}^{-}$, have been omitted from the diagram.

From Figure 6.16, it is possible for $\mathrm{ReO}_{4}^{-}$to reduced by the PANI-NCS to $\mathrm{ReO}_{3}$, or another rhenium oxide if the potential for the PANI-NCS composite is less than $+0.36 \mathrm{~V}$ (Equation (6.7)).

$$
\mathrm{PNS}+\mathrm{H}^{+}+\mathrm{e}^{-} \rightleftharpoons \mathrm{ES}
$$

This is significantly lower than the $+0.75 \mathrm{~V}$ value of this transition under standard 
state conditions (Table 6.1). However, the reduction potential of the pernigraniline to emeraldine transition is $\mathrm{pH}$ dependant, decreasing by $59.1 \mathrm{mV}$ with each increase of $\mathrm{pH}$ value (Equation (6.1)). Even so, at the $\mathrm{ReO}_{4}^{-}$and $\mathrm{H}^{+}$concentrations used in this study, the reduction of $\mathrm{ReO}_{4}^{-}$by emeraldine is not energetically favourable.

The situation changes in the composite material, where the PANI is in contact with the NCS. The basic nature of the NCS surface (which equilibrates with water until a pH of 10.5 is reached) means that, in the composite, the PANI exists in a basic environment. As such, it should have a significantly reduced reduction potential. At a $\mathrm{pH}$ value of 10.5 , the reaction in (6.7) has a reduction potential of $\mathrm{E}=+0.13 \mathrm{~V}$.

The presence of PANI reduces the degree to which calcium is leached from the composite material, even under acidic conditions (discussed in more detail in Section 6.2.5). As calcium is still retained in the composite, the NCS will maintain a basic surface and the PANI should exhibit a lower reduction potential, due to the lower $\mathrm{H}^{+}$concentration at the composite surface. This would allow for the emeraldine base composite to facilitate reduction of $\mathrm{ReO}_{4}^{-}$. The most thermodynamically favourable reaction is reduction of $\mathrm{ReO}_{4}^{-}$to $\mathrm{ReO}_{3}$, although $\mathrm{Re}_{2} \mathrm{O}_{3}$ and $\mathrm{ReO}_{2}$ can also be formed via direct reduction of $\mathrm{ReO}_{4}^{-}$within the $\mathrm{pH}$ range observed during isotherm measurements. 


\subsubsection{X-Ray Photoelectron Spectroscopy}

The X-ray photoelectron spectra of polyaniline as the emeraldine salt is shown in Figure 6.17, and the associated parameters for the modelled peaks are given in Table 6.4. It can be seen that the polyaniline sample prepared from ${ }^{15} \mathrm{~N}$ aniline exhibits slightly greater binding energies than the sample prepared from ${ }^{14} \mathrm{~N}$ aniline. The polyaniline composite materials, from which the spectra shown in Figures 6.18-6.20 were obtained, were likewise prepared from ${ }^{15} \mathrm{~N}$-PANI. It was hoped that ${ }^{15} \mathrm{~N}-\mathrm{NMR}$ could be performed on the composite materials, as ${ }^{15} \mathrm{~N}-\mathrm{NMR}$ is a well-known technique for the characterisation of polyaniline. ${ }^{200}$ Unfortunately, peak broadening of the nitrogen signal due to the low polyaniline content (10 wt \%) prevented usable data from being obtained. As such, XPS analysis was undertaken on the samples instead.

Table 6.4 Parameters for X-ray photoelectron spectra of polyaniline samples.

\begin{tabular}{|c|c|c|c|c|}
\hline Orbital & Centre/ eV & HWHM/ eV & Area $^{a}$ & Bonding Type $^{b}$ \\
\hline \multicolumn{5}{|c|}{${ }^{14} \mathrm{~N}$-PANI, Figure 6.17(a) } \\
\hline $\mathrm{C} 1 \mathrm{~s}$ & $\begin{array}{l}289.0 \\
286.6 \\
285.0 \\
283.4\end{array}$ & $\begin{array}{l}0.49 \\
0.62 \\
0.65 \\
1.20\end{array}$ & $\begin{array}{r}16.6 \\
19.1 \\
100.0 \\
23.2\end{array}$ & Carbon tape ${ }^{c}$ \\
\hline $\mathrm{N} 1 \mathrm{~s}$ & $\begin{array}{l}399.2 \\
398.3\end{array}$ & $\begin{array}{l}1.47 \\
0.69\end{array}$ & $\begin{array}{r}93.6 \\
100.0\end{array}$ & $\begin{array}{c}\mathrm{Ph}_{2} \mathrm{NH}^{\cdot+} \\
\mathrm{Ph}_{2} \mathrm{NH}\end{array}$ \\
\hline \multicolumn{5}{|c|}{${ }^{15} \mathrm{~N}-P A N I$, Figure 6.17(b) } \\
\hline $\mathrm{C} 1 \mathrm{~s}$ & $\begin{array}{l}288.9 \\
286.6 \\
285.0 \\
283.7\end{array}$ & $\begin{array}{l}0.59 \\
0.85 \\
0.72 \\
0.77\end{array}$ & $\begin{array}{r}15.6 \\
28.0 \\
100.0 \\
60.6\end{array}$ & Carbon tape \\
\hline $\mathrm{N} 1 \mathrm{~s}$ & $\begin{array}{l}400.3 \\
398.9\end{array}$ & $\begin{array}{l}1.52 \\
0.75\end{array}$ & $\begin{array}{r}72.9 \\
100.0\end{array}$ & $\begin{array}{c}\mathrm{Ph}_{2} \mathrm{NH}^{\cdot+} \\
\mathrm{Ph}_{2} \mathrm{NH}\end{array}$ \\
\hline
\end{tabular}

\footnotetext{
${ }^{a}$ Normalised based on the area of the largest peak within each sample.

${ }^{b}$ Where known.

${ }^{c}$ Used as a sample mount.
}

The XPS spectra of the unmodified NCS material are presented in Figure 6.19 and Table 6.5. The spectra are typical of $\mathrm{NCS}^{47}$ and other calcium silicates. ${ }^{201,202}$ The O 1s signal may be assigned to three chemical functionalities within the silicate structure: A bridging $\mathrm{Si}-\mathrm{O}-\mathrm{Si}$ signal (binding energy $532.0 \mathrm{eV}$ ), and two non-bridging oxygen 

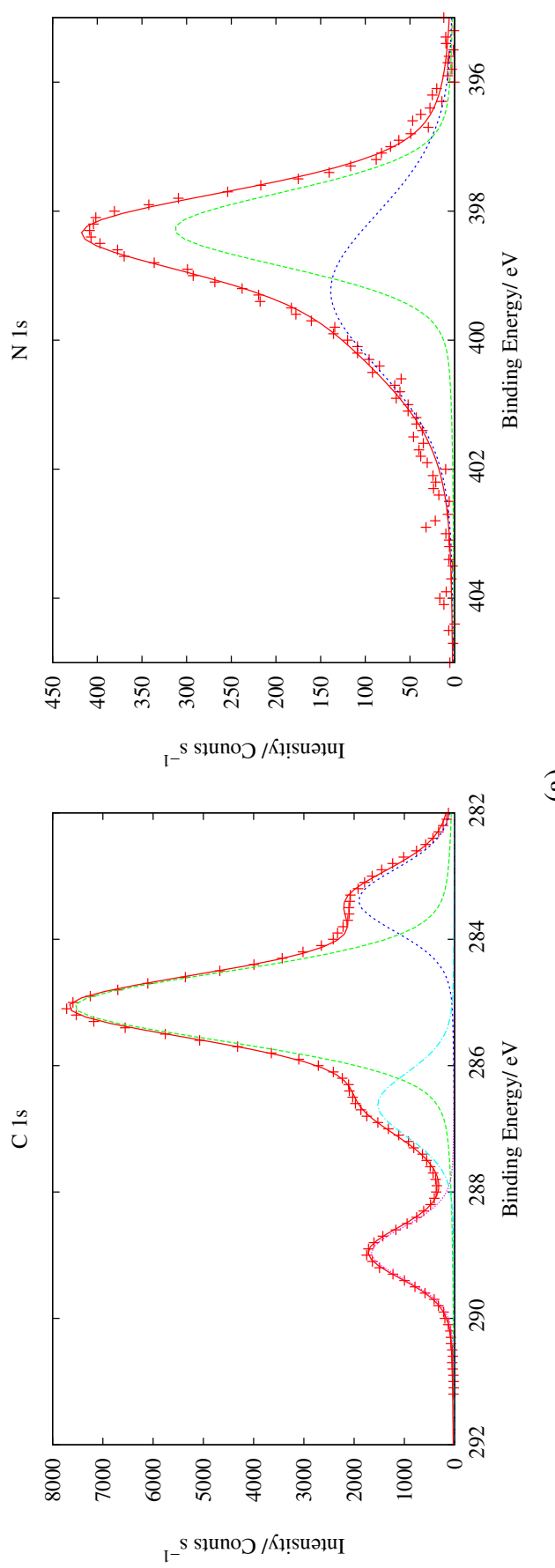

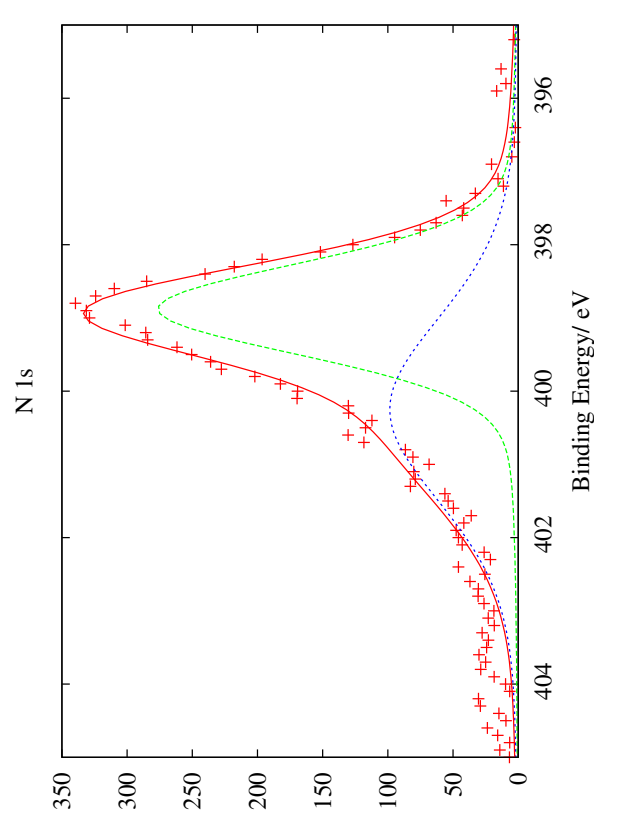

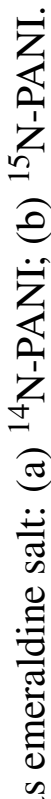

త

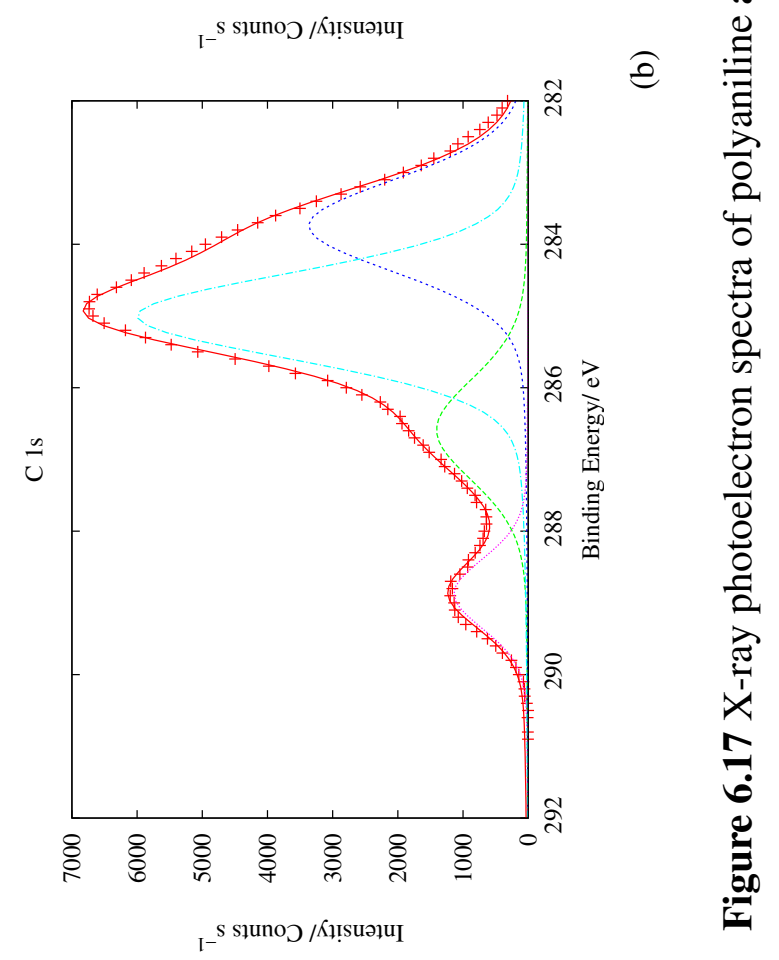


signals. One, at a binding energy of $530.7 \mathrm{eV}$ arises from the $\mathrm{Si}-\mathrm{O}-\mathrm{Ca}$ oxygen environment and the other arises from the interaction of the groups $\mathrm{Si}-\mathrm{OH}$ or $\mathrm{Ca}-\mathrm{OH}_{2}$ at a binding energy of $529.2 \mathrm{eV} .{ }^{47}$ The $\mathrm{Si} 2 \mathrm{p}$ signal varies with the degree of polymerisation of the silicate backbone. It is known that silicates with a lower degree of polymerisation exhibit lower Si $2 p$ binding energies. ${ }^{202} \mathrm{~A} \mathrm{Q}^{4}$ type signal occurs at a binding energy of $104.2 \mathrm{eV}$, a $\mathrm{Q}^{2}$ signal at $102.1 \mathrm{eV}$ and a $\mathrm{Q}^{1}$ signal at a binding energy of $100.9 \mathrm{eV}^{\dagger}$ Although the Si $2 p$ orbital should exhibit splitting into the $2 p_{\frac{3}{2}}$ and $2 p_{\frac{1}{2}}$ spin states, this is usually not resolved in a typical XPS spectrum. The Ca 2 p orbital can be deconvoluted to provide two peaks in the $2 \mathrm{p}_{\frac{3}{2}}$ spin state. These are most likely due to the presence of $\mathrm{SiO}-\mathrm{Ca}-\mathrm{OSi}$ and $\mathrm{Si}-\mathrm{O}-\mathrm{Ca}-\mathrm{OH}_{2}$ chemical environments for the calcium.

When the XPS spectra of the PANI-NCS composite (Figure 6.18, Table 6.5) are compared to those of pure NCS (Figure 6.19, Table 6.5) and pure PANI (Figure 6.17, Table 6.4), several differences can be observed. It can be seen that the $\mathrm{N} 1 \mathrm{~s}$ signal attributable to the $\mathrm{NH}^{\cdot+}$ at a binding energy of $400.3 \mathrm{eV}$ is not present in the $\mathrm{N} 1$ s spectrum of the composite material, indicative of the deprotonation of PANI (initially emeraldine salt) to emeraldine base in the composite material. Due to the low $\mathrm{N}$ 1s signal intensity in the composite material, signals resulting from amine and imine functionalities present in emeraldine base could not be deconvoluted. It can also be seen that the $\mathrm{N} 1 \mathrm{~s}$ signal attributable to the amine groups has shifted from $398.9 \mathrm{eV}$ in the unmodified PANI to $398.3 \mathrm{eV}$ in the composite material. This decrease in binding energy indicates a greater degree of negative charge in the nitrogen chemical environment in the composite material.

As it has been established through UV-visible spectrographic studies that the PANI binds to the NCS through hydrogen bonding, the downward shift in the N 1s binding energy can be explained should the hydrogen bonding occur between an amine hydrogen on the PANI and an oxygen within the NCS (Figure 6.11(a)). This would contribute additional negative charge to the PANI nitrogen. Should the hydrogen bonding occur between an NCS hydroxyl hydrogen and a PANI nitrogen (Figures 6.11(b)-6.11(c)), an upward shift in the $\mathrm{N}$ 1s binding energy would be expected due to the presence of additional positive

${ }^{\dagger}$ The $\mathrm{Q}^{\mathrm{n}}$ notation refers to the degree of polymerisation of $\mathrm{SiO}_{4}$ units. Thus, $\mathrm{Q}^{4}$ is indicative of the threedimensional $\mathrm{SiO}_{2}$ network, $\mathrm{Q}^{3}$ is represented by $\left(\mathrm{SiO}_{2}\right)_{\mathrm{n}}^{\mathrm{n}-}$ sheets, $\mathrm{Q}^{2}$ indicates $\left(\mathrm{SiO}_{3}\right)_{\mathrm{n}}^{2 \mathrm{n}-}$ chains and $\mathrm{Q}^{1}$ is exemplified by the $\mathrm{Si}_{2} \mathrm{O}_{7}^{6-}$ dimer, or the terminal $\mathrm{SiO}_{4}$ units of a $\mathrm{Q}^{2}$ chain. $\mathrm{Q}^{0}$ is the $\mathrm{SiO}_{4}^{4-}$ monomer. 
charge from the hydrogen. If the hydrogen bonding between PANI and NCS causes a decrease in the $\mathrm{N}$ 1s binding energy, a corresponding increase in the binding energy of the $\mathrm{O}$ 1s electrons should be observed. When comparing the $\mathrm{O} 1 \mathrm{~s}$ binding energy in Table 6.5, it can be seen that the composite material exhibits an increased $\mathrm{O} 1 \mathrm{~s}$ binding energy of between 1.0 to $1.2 \mathrm{eV}$ over the unmodified material. This is consistent with the expected increase in binding energy occurring as a result of hydrogen bonding between the PANI and NCS.

Table 6.5 Parameters for X-ray photoelectron spectra of PANI-NCS composite materials. Orbital Centre/ eV HWHM/ eV Area ${ }^{a} \quad$ Bonding Type $^{b}$

\begin{tabular}{|c|c|c|c|c|}
\hline \multicolumn{5}{|c|}{${ }^{15}$ N-PANI-NCS composite, Figure 6.18} \\
\hline \multirow[t]{3}{*}{$\mathrm{C} 1 \mathrm{~s}$} & 289.0 & 1.01 & 31.9 & \\
\hline & 286.3 & 0.98 & 64.3 & \\
\hline & 285.0 & 0.81 & 100 & Carbon tape $^{c}$ \\
\hline $\mathrm{N} 1 \mathrm{~s}$ & 398.3 & 1.89 & 100 & $\mathrm{Ph}_{2} \mathrm{NH}$ \\
\hline \multirow[t]{3}{*}{$\mathrm{O} 1 \mathrm{~s}$} & 533.2 & 1.26 & 68.7 & Bridging $\mathrm{Si}-\mathrm{O}-\mathrm{Si}$ \\
\hline & 531.7 & 1.00 & 100 & Non-bridging $\mathrm{Si}-\mathrm{O}-\mathrm{Ca}$ \\
\hline & 530.2 & 0.65 & 35.4 & $\begin{array}{c}\text { Non-bridging } \\
\mathrm{Si}-\mathrm{OH} \text { or } \mathrm{Ca}-\mathrm{OH}_{2}\end{array}$ \\
\hline \multirow[t]{3}{*}{ Si $2 p$} & 103.2 & 0.73 & 52.4 & $\mathrm{Q}^{4} \mathrm{Si}$ \\
\hline & 102.3 & 0.60 & 100 & $\mathrm{Q}^{2} \mathrm{Si}$ \\
\hline & 101.5 & 0.60 & 89.7 & $\mathrm{Q}^{1} \mathrm{Si}$ \\
\hline $\mathrm{Ca} 2 \mathrm{p}_{\frac{1}{2}}$ & 350.5 & 0.86 & 94.7 & \\
\hline \multirow{2}{*}{$\mathrm{Ca} 2 \mathrm{p}_{\frac{3}{2}}^{2}$} & 347.4 & 0.75 & 100 & \\
\hline & 346.7 & 0.66 & 93.1 & \\
\hline \multicolumn{5}{|c|}{ 2-ethoxyethanol washed NCS, Figure 6.19} \\
\hline \multirow[t]{3}{*}{$\mathrm{O} 1 \mathrm{~s}$} & 532.0 & 1.33 & 96.9 & Bridging \\
\hline & 530.7 & 1.05 & 100.0 & Non-bridging \\
\hline & 529.2 & 0.75 & 43.8 & Non-bridging \\
\hline \multirow[t]{3}{*}{$\mathrm{Si} 2 \mathrm{p}$} & 104.2 & 1.27 & 53.7 & $\mathrm{Q}^{4} \mathrm{Si}$ \\
\hline & 102.1 & 1.02 & 100 & $\mathrm{Q}^{2} \mathrm{Si}$ \\
\hline & 100.9 & 0.85 & 88.8 & $\mathrm{Q}^{1} \mathrm{Si}$ \\
\hline $\mathrm{Ca} 2 \mathrm{p}_{\frac{1}{2}}$ & 349.6 & 1.55 & 100 & \\
\hline \multirow[t]{2}{*}{$\mathrm{Ca} 2 \mathrm{p}_{\frac{3}{2}}^{2}$} & 346.7 & 0.79 & 68.1 & \\
\hline & 345.7 & 0.68 & 64.7 & \\
\hline
\end{tabular}

\footnotetext{
${ }^{a}$ Normalised based on the area of the largest peak within each sample.

${ }^{b}$ Where known.

${ }^{c}$ Used as a sample mount.
}

When comparing the XPS spectra of the PANI-NCS material prior to $\mathrm{ReO}_{4}^{-}$sorption 

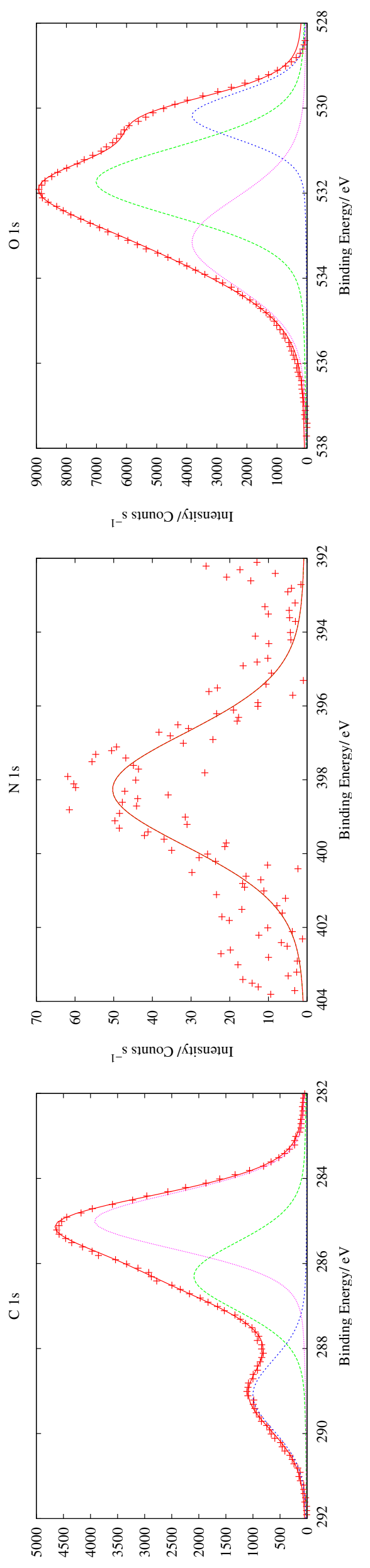

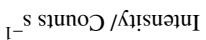
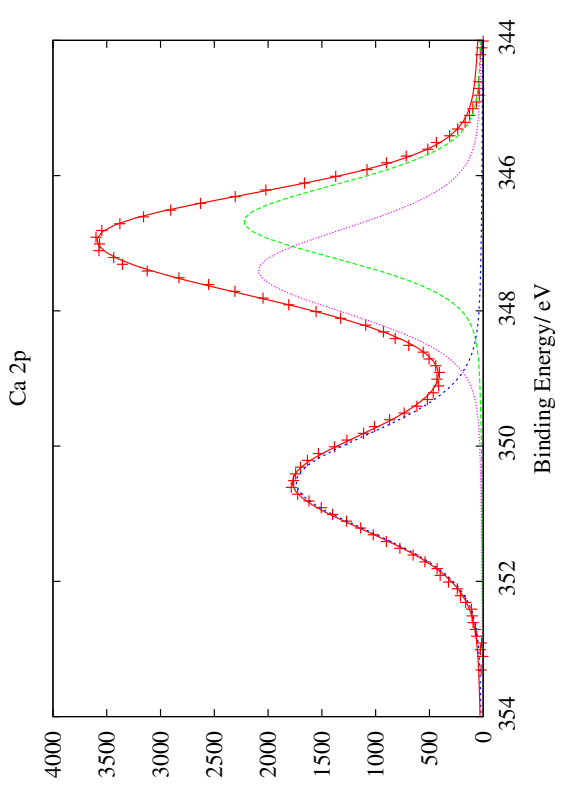

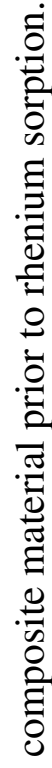

U

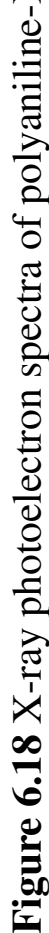



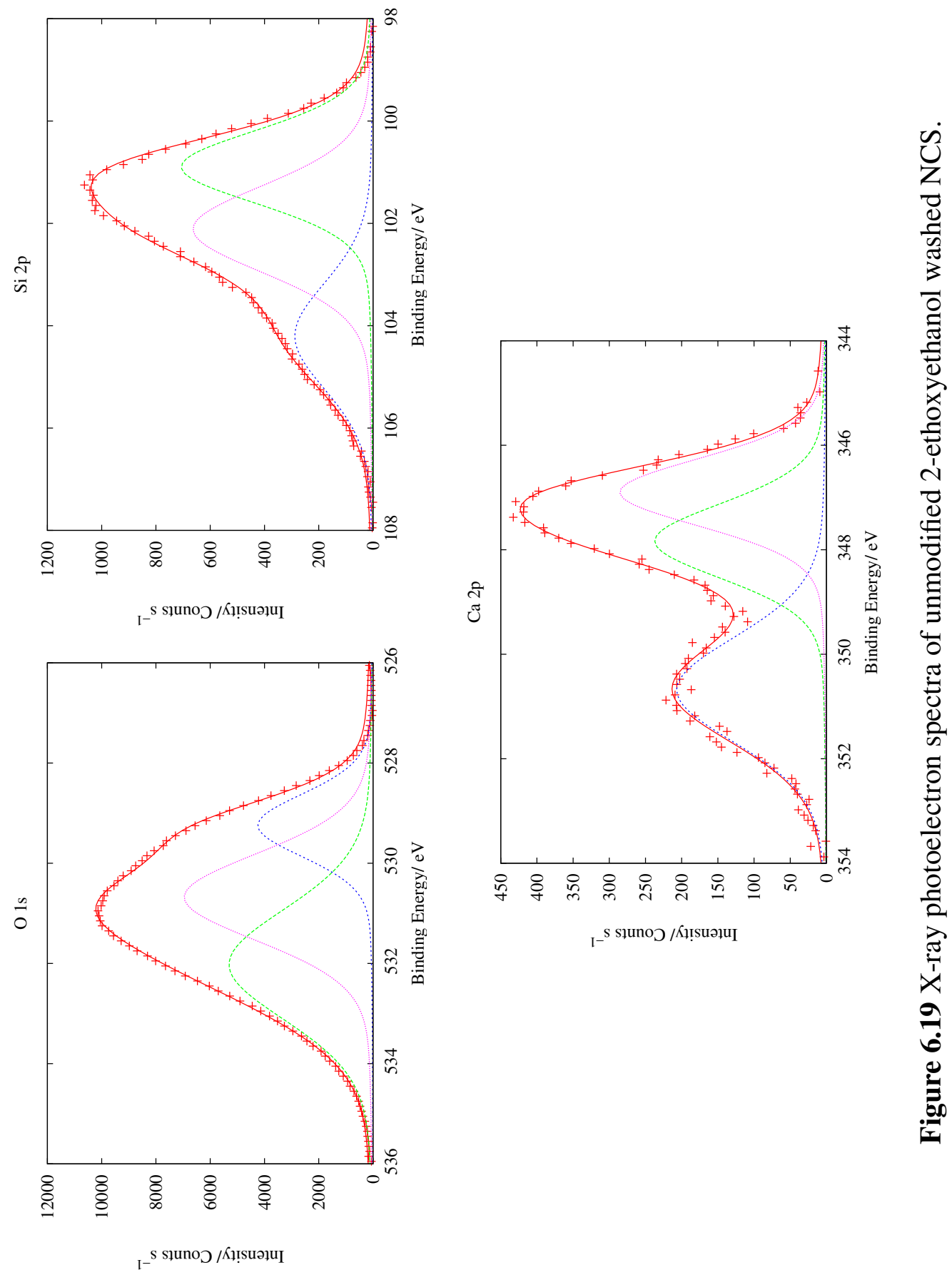

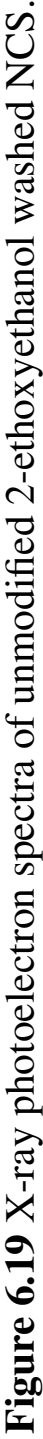


(Figure 6.18, Table 6.5), and subsequent to sorption (Figure 6.20, Table 6.6), several changes are evident. It can be seen that the binding energy of the $\mathrm{O} 1 \mathrm{~s}$ and $\mathrm{Si} 2 \mathrm{p}$ electrons has increased by approximately $1 \mathrm{eV}$, whereas the binding energy of the $\mathrm{N} 1 \mathrm{~s}$ electrons has decreased by $0.6 \mathrm{eV}$. This is indicative of a greater negative charge on the nitrogen, and greater positive charge on the silicon and oxygen. The binding energy of the Re $4 \mathrm{f}$ electrons has also significantly changed: from a binding energy of $46.3 \mathrm{eV}$ for the most intense $\mathrm{Re}_{4} \mathrm{f}_{\frac{7}{2}}$ signal in $\mathrm{KReO}_{4}$ (Figure 6.21) to an energy of $42.3 \mathrm{eV}$ for the equivalent peak in the sorbed material. This decrease in binding energy of $4 \mathrm{eV}$ is indicative of a decrease in the oxidation state of rhenium. Stable rhenium species which exhibit binding energies either side of this $42.3 \mathrm{eV}$ value are: Re metal, which has a $4 \mathrm{f}_{\frac{7}{2}}$ binding energy of $40.3 \mathrm{eV}$, and $\mathrm{ReO}_{2}$, which has a $4 \mathrm{f}_{\frac{7}{2}}$ binding energy of $43.6 \mathrm{eV} .{ }^{203}$ An oxide of rhenium in the +3 oxidation state is known to exist, as $\operatorname{Re}_{2} \mathrm{O}_{3} \cdot{ }^{204}$ However, this species is not commonly observed in aqueous media. ${ }^{193,204}$ It is possible that $\operatorname{Re}_{2} \mathrm{O}_{3}$ species formed as a consequence of sample preparation, or the high-vacuum conditions under which XPS measurements are conducted. However, under the reaction conditions used for the rhenium sorption, it is possible that $\mathrm{Re}_{2} \mathrm{O}_{3}$ is formed. This is discussed further in Section 6.2.3.

For $\mathrm{ReO}_{4}^{-}$to be reduced to $\mathrm{Re}_{2} \mathrm{O}_{3}$, it would be expected that the polyaniline should be oxidised to the pernigraniline state. This would result in an increase of the observed binding energy of the $\mathrm{N} 1 \mathrm{~s}$ electrons due to the oxidation of the amine nitrogen to an imine. As an $\mathrm{N}$ 1s binding energy decrease is observed instead, the nitrogen moieties are more likely to have formed some sort of hydrogen bonding-equivalent interaction between the nitrogen and rhenium atoms masking any potential change in the nitrogen signal.

XPS studies of rhenium-nitrogen interactions during the formation of rhenium nitride films have been reported by Soto. ${ }^{205}$ It was observed that an increase in the ratio of $\mathrm{N}: \mathrm{Re}$ was accompanied by an increase in the Re $4 \mathrm{f}$, and a decrease in the $\mathrm{N} 1 \mathrm{~s}$ binding energies when compared to that of their zero-valent elemental states. For a film with a composition of $\operatorname{Re}_{3} \mathrm{~N}_{4}$ binding energies of $41.8 \mathrm{eV}\left(\operatorname{Re} 4 \mathrm{f}_{\frac{7}{2}}\right)$ and $397.2 \mathrm{eV}(\mathrm{N} 1 \mathrm{~s})$ were reported. XPS analysis of a series of chloro-amine complexes of the form $\left[\mathrm{ReCl}_{2} \cdot \mathrm{L}\right]$ (with $\mathrm{L}$ being pyridine of a similar nitrogen ligand) has been reported by Tisley and Walton. ${ }^{206}$ Binding 

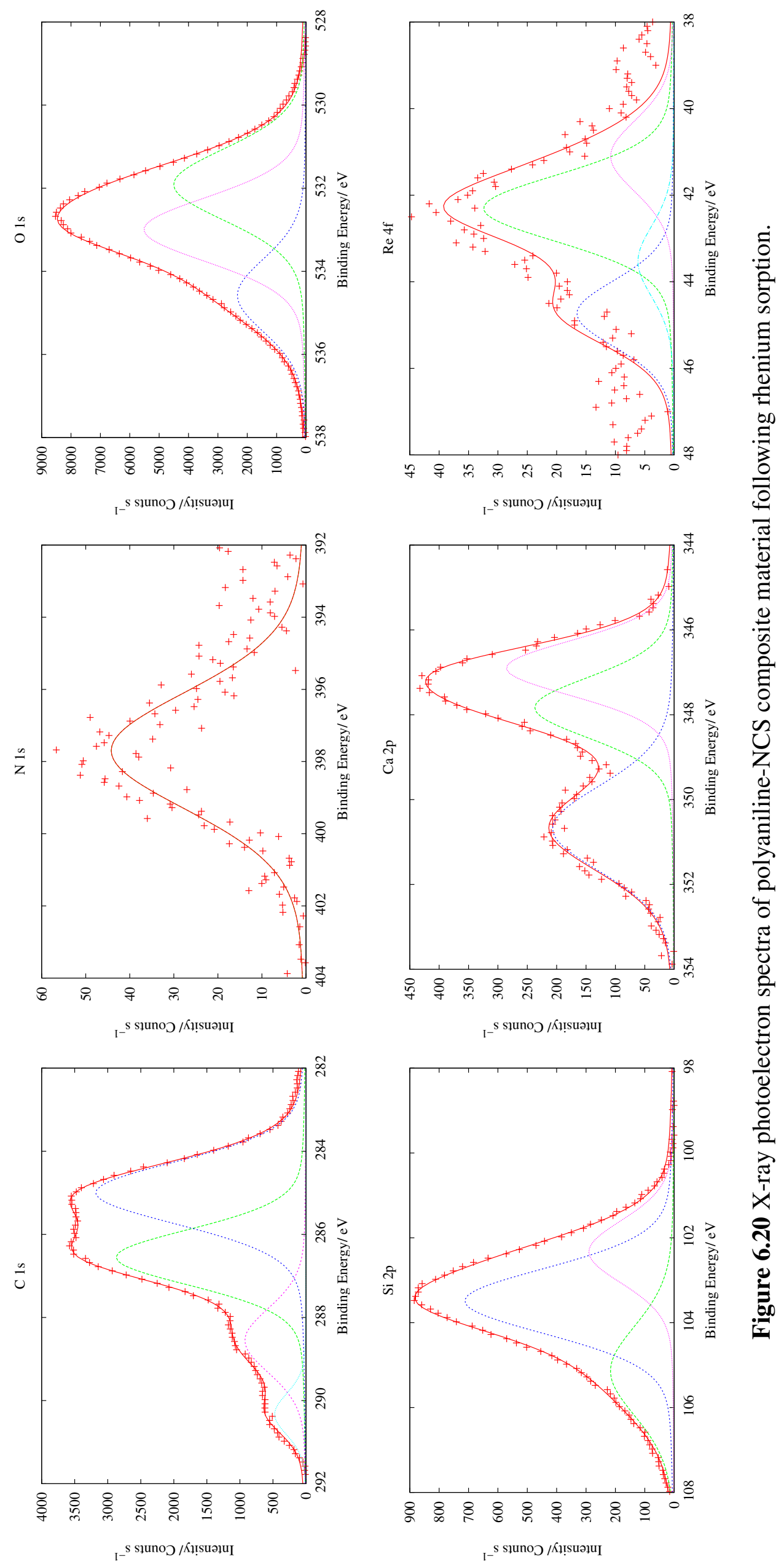

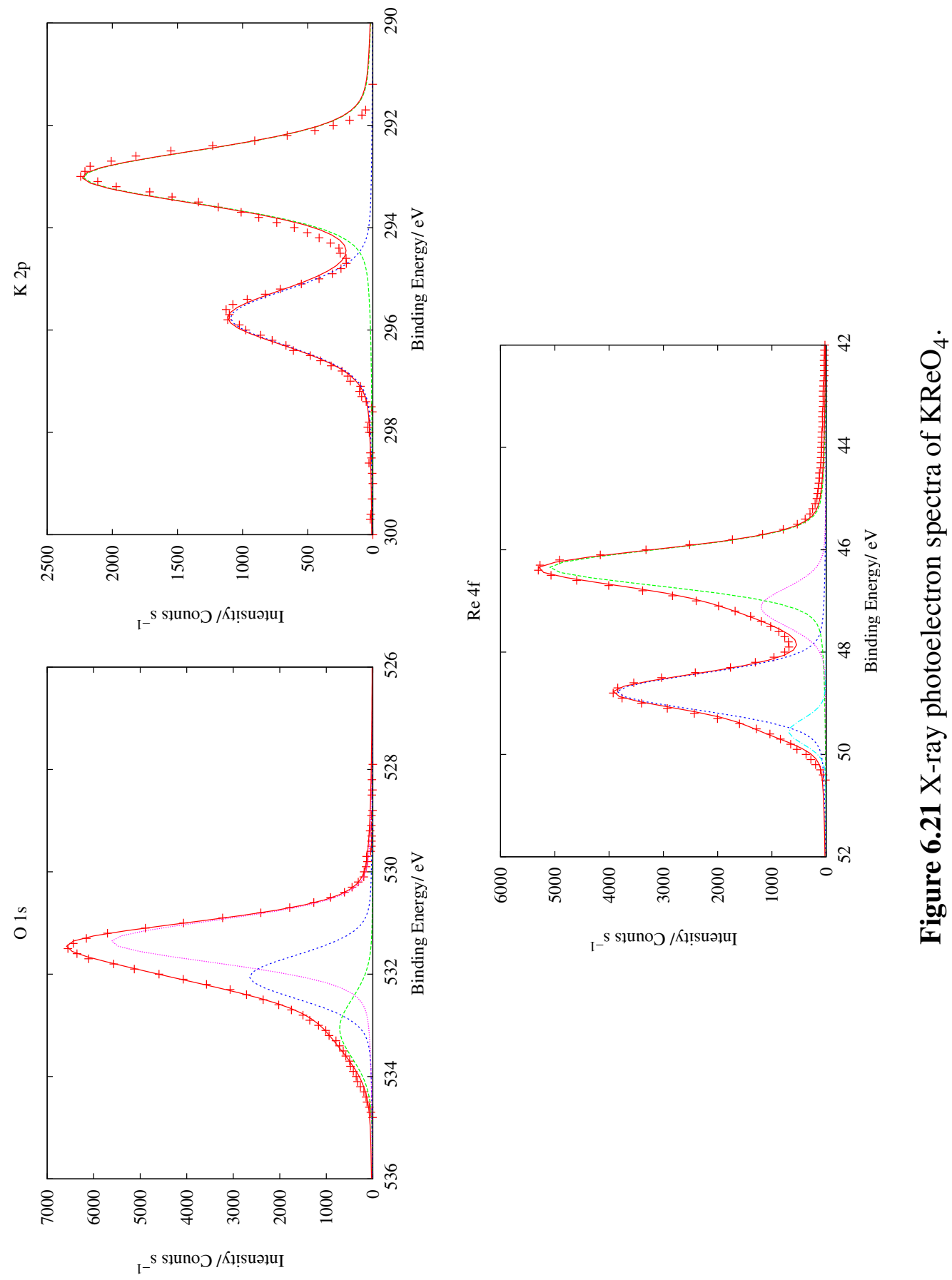
Table 6.6 Parameters for X-ray photoelectron spectra of PANI-NCS composite following rhenium sorption from a $\mathrm{KReO}_{4}$ solution.

Orbital Centre/ eV HWHM/ eV Area $^{a}$ Bonding Type ${ }^{b}$

\begin{tabular}{|c|c|c|c|c|}
\hline \multicolumn{5}{|c|}{ PANI-NCS following $\mathrm{KReO}_{4}$ sorption, Figure 6.20} \\
\hline \multirow[t]{4}{*}{$\mathrm{C} 1 \mathrm{~s}$} & 290.3 & 0.60 & 9.8 & \\
\hline & 288.6 & 0.94 & 29.9 & \\
\hline & 286.5 & 0.81 & 80.4 & \\
\hline & 285.0 & 0.91 & 100 & Carbon tape \\
\hline $\mathrm{N} 1 \mathrm{~s}$ & 397.7 & 1.97 & 100 & \\
\hline \multirow[t]{3}{*}{$\mathrm{O} 1 \mathrm{~s}$} & 534.6 & 1.07 & 48.3 & Bridging \\
\hline & 532.0 & 0.94 & 100 & Non-bridging \\
\hline & 531.9 & 1.04 & 91.3 & Non-bridging \\
\hline \multirow[t]{3}{*}{ Si $2 p$} & 105.1 & 1.29 & 41.8 & $\mathrm{Q}^{4} \mathrm{Si}$ \\
\hline & 103.5 & 0.94 & 100 & $\mathrm{Q}^{2} \mathrm{Si}$ \\
\hline & 102.5 & 0.90 & 39.1 & $\mathrm{Q}^{1} \mathrm{Si}$ \\
\hline $\mathrm{Ca} 2 \mathrm{p}_{\frac{1}{2}}$ & 350.7 & 1.22 & 100 & \\
\hline \multirow{2}{*}{$\mathrm{Ca} 2 \mathrm{p}_{\frac{3}{2}}^{2}$} & 347.8 & 0.86 & 81.5 & \\
\hline & 346.9 & 0.78 & 88.8 & \\
\hline \multirow{2}{*}{$\operatorname{Re} 4 f_{\frac{5}{2}}$} & 44.7 & 0.93 & 51.0 & \\
\hline & 43.5 & 1.00 & 20.3 & \\
\hline \multirow{2}{*}{$\operatorname{Re} 4 f_{\frac{7}{2}}$} & 42.3 & 0.93 & 100 & \\
\hline & 41.1 & 1.00 & 35.7 & \\
\hline \multicolumn{5}{|c|}{$\mathrm{KReO}_{4}$, Figure 6.21} \\
\hline \multirow[t]{3}{*}{$\mathrm{O} 1 \mathrm{~s}$} & 533.1 & 0.71 & 18.3 & \\
\hline & 532.1 & 0.52 & 49.9 & \\
\hline & 531.4 & 0.49 & 100 & \\
\hline $\mathrm{K} 2 \mathrm{p}_{\frac{1}{2}}$ & 295.8 & 0.65 & 51.9 & \\
\hline $\mathrm{K} 2 \mathrm{p}_{\frac{3}{2}}^{2}$ & 293.0 & 0.62 & 100 & \\
\hline \multirow[t]{2}{*}{$\operatorname{Re} 4 f_{\frac{5}{2}}$} & 49.6 & 0.30 & 9.6 & $\operatorname{Re}_{2} \mathrm{O}_{7}$ \\
\hline & 48.8 & 0.42 & 75.7 & $\mathrm{Re}_{2} \mathrm{O}_{7}$ \\
\hline \multirow{2}{*}{$\operatorname{Re} 4 f_{\frac{7}{2}}$} & 47.2 & 0.45 & 25.1 & $\operatorname{Re}_{2} \mathrm{O}_{7}$ \\
\hline & 46.3 & 0.42 & 100 & $\mathrm{ReO}_{4}^{-}$ \\
\hline
\end{tabular}

${ }^{a}$ Normalised based on the area of the largest peak within each sample.

${ }^{b}$ Where known. 
energies of the $\operatorname{Re} 4 \mathrm{f}_{\frac{7}{2}}$ electrons ranged from $41.8 \mathrm{eV}$ to $42.5 \mathrm{eV}$.

The measured binding energies for the PANI-NCS composites after rhenium sorption are more closely related to those for $\mathrm{Re}-\mathrm{N}$ interactions than for $\mathrm{Re}-\mathrm{O}$ interactions. Any potential oxygen ligands of the rhenium species are not detected by XPS analysis; the signal would be masked by that of the NCS oxygen.

The sorbed rhenium is most likely present in the +3 oxidation state, with some form of covalent interaction between the $\mathrm{N}$ atoms of the polyaniline, and the Re. It is unlikely to be strictly a rhenium nitride due to the oxic, aqueous conditions under which the sorption was performed. Thus, the species is most likely a $\mathrm{Re}_{x} \mathrm{O}_{y}(\mathrm{~N}-\mathrm{PANI})_{z}$ compound. Although few reports exist of aniline acting as a ligand, both cobalt ${ }^{207}$ and rhenium ${ }^{208}$ complexes of aniline are known. Polyaniline is also known to act as a ligand, ${ }^{209}$ as the imine nitrogen in PANI is similar in nature to that of pyridine and other conjugated imines. Examples of rhenium-oxy-nitrogen compounds have been previously reported. Johnson et al., for example have reported crystal structures of many rhenium oxide salts, in which the oxidation state of the rhenium varies from +2 to +7 , where the rhenium oxide crystallises with pyridine as a solvent of crystallisation. ${ }^{210}$ Rhenium oxides in the +4 oxidation state have also been reported as a major product in the electroreduction of $\mathrm{ReO}_{4}^{-}$on noble metals. $^{211}$

Elements in the $5 \mathrm{~d}$ transition series, to which rhenium belongs, have a very high electron density. It is probable that the presence of increased electron density in close proximity to the PANI-NCS material is responsible to some degree for the observed shifts in the electron binding energies subsequent to $\mathrm{ReO}_{4}^{-}$sorption. ${ }^{203}$

Although XPS demonstrated that the $\mathrm{ReO}_{4}^{-}$was unequivocally reduced, identification of the species oxidised concurrently could not be made by XPS analysis. If the polyaniline were oxidised an increase in the N 1s binding energy should have been observed, which was not the case. It may be possible that the PANI-NCS composite facilitated oxidation of $\mathrm{Cl}^{-}$within the system to $\mathrm{Cl}_{2}$, or the oxidation of surface-bound hydroxyl species to peroxides or $\mathrm{O}_{2}$ gas. However, this is highly unlikely due to the high reduction potential of $\mathrm{Cl}^{-}$or $\mathrm{OH}^{-}$when compared to $\mathrm{ReO}_{4}^{-}$. Oxidation of the carbon backbone of polyaniline is 
another possibility, but this should not occur without first oxidising the polyaniline amine moieties to imine.

\subsubsection{Rhenium Sorption Isotherms}

Figure 6.22 and Table 6.7 show the sorption of $\mathrm{ReO}_{4}^{-}$by a polyaniline-NCS composite material. It can be seen from the table that the data is best modelled by the Langmuir isotherm, having an $R^{2}$ value of 0.984 . The degree of conformity to the Langmuir isotherm is greater than that to either the Freundlich isotherm $\left(R^{2}=0.814\right)$ or the Tempkin isotherm $\left(R^{2}=0.938\right)$. The maximum uptake capacity for this material is $0.58 \mathrm{mmol} \mathrm{Re} \mathrm{g}^{-1}$ composite. The degree of calcium leaching (and solution $\mathrm{pH}$ ) as a result of this rhenium sorption is shown in Figure 6.23. It can be seen from this figure that, although some calcium is leached from the PANI-NCS composite, the leaching occurs to a lesser degree than from the unmodified NCS material. The greatest degree of calcium leaching observed with this sample resulted in an equilibrium $\mathrm{Ca}^{2+}$ concentration of $0.922 \pm 0.002 \mathrm{mmol} \mathrm{dm}^{-3}$. This is significantly less than the amount of calcium leached from the material during copper sorption $\left(\left[\mathrm{Ca}^{2+}\right]\right.$ in excess of $\left.6 \mathrm{mmol} \mathrm{dm}^{-3}\right)$ or even in equilibration with water $\left(\left[\mathrm{Ca}^{2+}\right]=1.33 \pm 0.03 \mathrm{mmol} \mathrm{dm}{ }^{-3}\right){ }^{\dagger}$ When comparing the amount of calcium leached from NCS with the equilibrium rhenium concentration, an interesting trend is apparent. The degree of calcium leaching increases with an increase in the amount of nonsorbed rhenium until a maximum is reached, whereupon the degree of calcium leaching decreases. The maximum amount of calcium leaching occurs at the point where the PANI-NCS reaches saturation sorption of perrhenate. The following may provide an explanation for this:

- When no rhenium is present in the system, the PANI protects the NCS from the acidic conditions, retarding calcium leaching.

- As the concentration of perrhenate is increased, its initial interaction with the PANI weakens the bonding between the PANI and NCS, leading to an increase

\footnotetext{
${ }^{\dagger}$ For all these samples, the NCS was applied at a loading of $1 \mathrm{~g} \mathrm{dm}^{-3}$.
} 


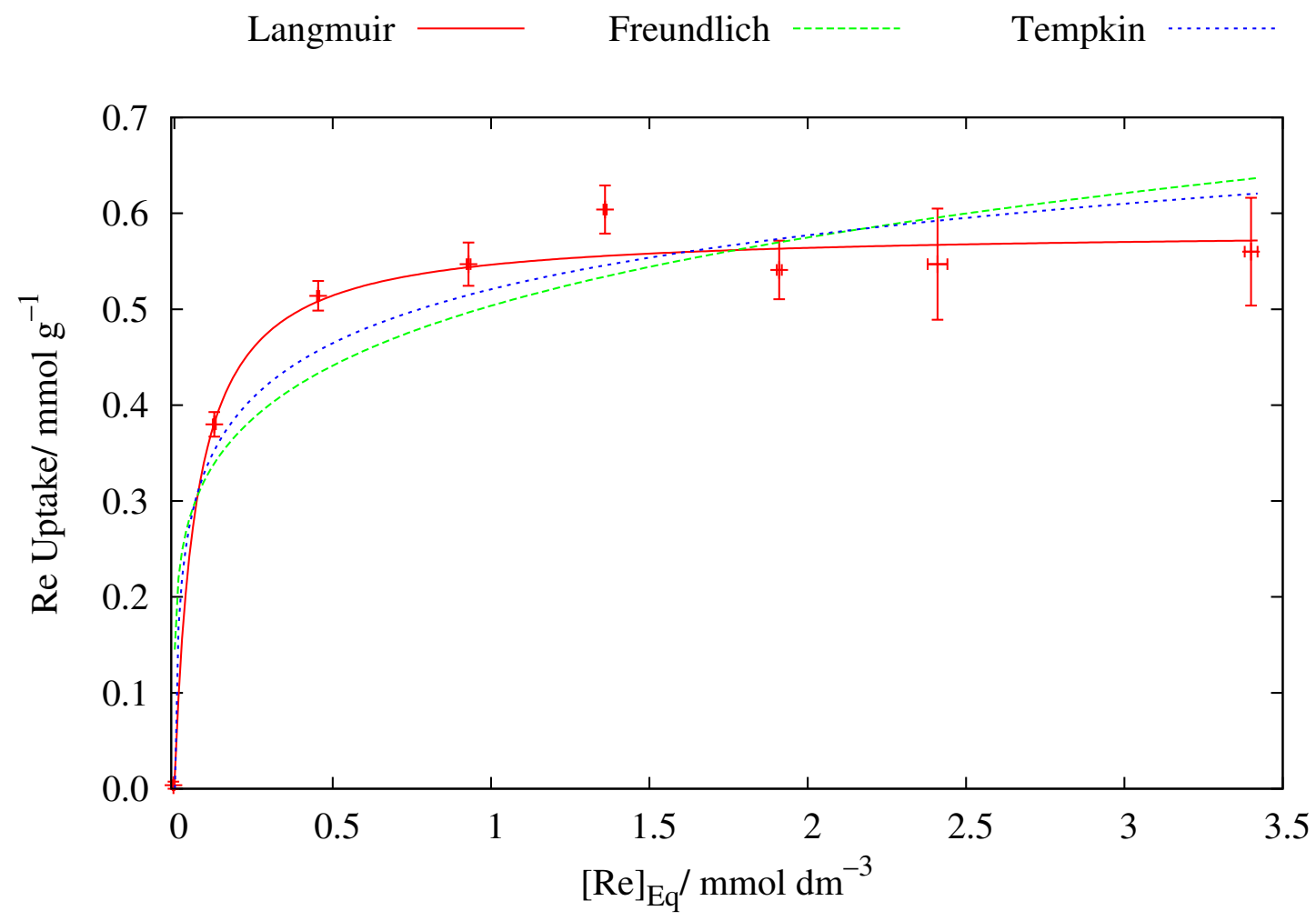

Figure 6.22 Isotherm models for the sorption of $\mathrm{ReO}_{4}^{-}$by PANI-NCS composite under acidic conditions.

Table 6.7 Parameters for perrhenate sorption isotherms as shown in Figure 6.22.

\begin{tabular}{lr}
\hline Parameter & Value \\
\hline \multicolumn{2}{c}{ Langmuir Isotherm } \\
\hline$n_{\infty}{ }^{a}$ & 0.583 \\
$K^{b}$ & 15.0 \\
$R^{2}$ & 0.984 \\
\hline \multicolumn{2}{l}{ Freundlich Isotherm } \\
\hline$n_{\infty} a$ & 0.504 \\
$C_{2}$ & 5.24 \\
$R^{2}$ & 0.814 \\
\hline \multicolumn{2}{c}{ Tempkin Isotherm } \\
\hline$n_{\infty} C_{1}$ & $8.12 \times 10^{-2}$ \\
$C_{2}$ & 612 \\
$R^{2}$ & 0.938 \\
\hline
\end{tabular}

${ }^{a}$ Presented in dimensions of $\mathrm{mmol} \mathrm{g}^{-1}$.

${ }^{b}$ Presented in dimensions of $\mathrm{dm}^{3} \mathrm{mmol}^{-1}$. 
in the amount of calcium leached from the material. The weakening of the bond between PANI and NCS may also be indicative of an oxidation of the PANI to the pernigraniline form.

- The perrhenate is reduced to a rhenium oxide layer, forming a protective layer on the surface of the NCS and preventing further calcium leaching.

- When a significant excess of perrhenate is present, this protecting layer forms quickly, reducing the total quantity of calcium leached.

The observed decrease in the degree of calcium leaching at increasing rhenium concentrations is unlikely to be caused by interference in the quantitative analysis of calcium. It can be seen that an increase in the observed degree of calcium leaching is accompanied by an increase in the $\mathrm{pH}$ value of the solution, behaviour consistent with other observations made of the NCS material, as was noted in, for example Chapter 3, Figure 3.4.
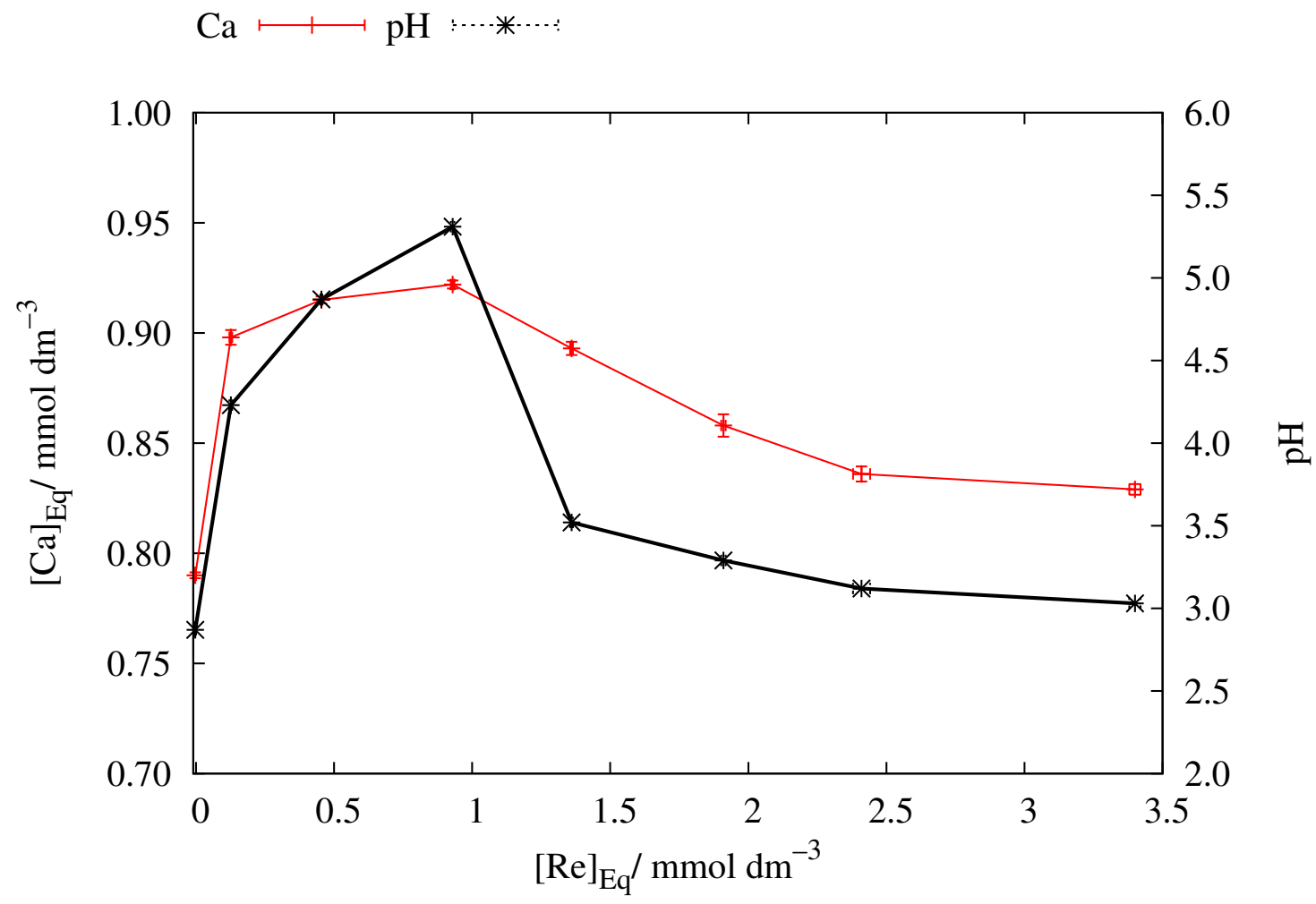

Figure 6.23 Supernatant $\left[\mathrm{Ca}^{2+}\right]$ and $\mathrm{pH}$ for the $\mathrm{ReO}_{4}^{-}$sorption isotherm presented in Figure 6.22.

The composite material contains nominally $10 \mathrm{wt} \%$ PANI, or $0.25 \mathrm{mmol} \mathrm{g}^{-1}$ assuming an empirical formula for the polyaniline of $\mathrm{C}_{24} \mathrm{H}_{18} \mathrm{~N}_{4}$ (emeraldine base). The transition 
of emeraldine to pernigraniline is a two electron oxidation based on this repeat unit (Figure 6.1). Thus, if the $\mathrm{ReO}_{4}^{-}$is reduced to $\mathrm{ReO}_{2}$, a three electron reduction, it would be expected that total oxidation of the emeraldine would result in the reduction of $0.17 \mathrm{mmol} \mathrm{ReO}_{4}^{-} \mathrm{g}^{-1}$ of composite. If $\mathrm{Re}_{2} \mathrm{O}_{3}$ were formed, a four electron transition, sorption of $0.13 \mathrm{mmol} \mathrm{ReO}_{4}^{-} \mathrm{g}^{-1}$ would be expected. However, if $\mathrm{ReO}_{3}$ were formed through reduction of $\mathrm{ReO}_{4}^{-}$by PANI, then a sorption capacity of $0.50 \mathrm{mmol} \mathrm{ReO}_{4}^{-} \mathrm{g}^{-1}$ would result. Although this is consistent with the measured sorption capacity, there is no evidence for the formation of $\mathrm{ReO}_{3}$ in XPS studies. If reduction of $\mathrm{ReO}_{4}^{-}$to $\mathrm{Re}_{2} \mathrm{O}_{3}$ was occurring, the PANI-NCS would be sorbing more than four times as much $\mathrm{ReO}_{4}^{-}$as could be accounted for by a redox mechanism. This discrepancy may be accounted for by considering the nature of the XPS measurement.

The XPS technique detects electrons emitted from a sample upon exposure to X-ray radiation. As a free electron will readily react with nearby atoms, only electrons ejected from atoms within a few nanometres of the sample surface are detected and analysed by the instrument. ${ }^{203}$ For this reason, although $\mathrm{Re}_{2} \mathrm{O}_{3}$ was identified as the predominant rhenium species in the spent sorbent, this may not be the only species present. It is possible that $\mathrm{Re}_{2} \mathrm{O}_{3}$ is only formed as a surface layer on another rhenium species. Thus, the PANI-NCS composite can initially reduce the $\mathrm{ReO}_{4}^{-}$to the thermodynamically preferred $\mathrm{ReO}_{3}$ phase. Subsequently, formation of a surface layer of $\mathrm{Re}_{2} \mathrm{O}_{3}$ can form. This can occur either by direct reduction of $\mathrm{ReO}_{4}^{-}$or reduction of $\mathrm{ReO}_{3}$, as both routes are viable according to Figure 6.16.

The observed sorption capacity of the composite material is consistent with a reduction mechanism if the majority of the sorbed rhenium were present as $\mathrm{ReO}_{3}$, with a small quantity of $\mathrm{Re}_{2} \mathrm{O}_{3}$ also formed.

\subsection{Conclusions}

Polyaniline-NCS composite materials were successfully prepared by coating NCS with polyaniline post synthesis of the constituent components. The polyaniline thus treated 
remained in the emeraldine oxidation state, being present in the composite material as the base form. A red-shift in the $\mathrm{n} \rightarrow \pi^{*}$ transition present in the UV-visible reflectance spectrum was of a magnitude consistent with hydrogen bonding interactions between the amine hydrogen of the PANI and the oxygen present in the NCS structure. Changes to the redox characteristics of the composite material were ill defined. This was most likely due to the nature of the composite material: as a powder, adequate electrical connections between it and the available cyclic voltammetry instrument could not be made. X-ray photoelectron spectroscopy did provide indirect evidence for the polymer retaining its redox-activity, as changes in the redox state of rhenium following $\mathrm{ReO}_{4}^{-}$ sorption could clearly be detected. However, the expected oxidation of PANI to the pernigraniline oxidation state was not observed. The PANI-NCS composite material exhibited significantly enhanced stability under acidic conditions when compared to the unmodified form. While calcium was leached from the composite material under acidic conditions, the degree of leaching was approximately one sixth that observed for the unmodified material under comparable conditions. Composite materials containing up to $10 \mathrm{wt} \%$ of the polymer could be easily prepared.

Studies on the sorption of $\mathrm{ReO}_{4}^{-}$by polyaniline films demonstrated that, prima facie, $\mathrm{ReO}_{4}^{-}$is unable to oxidise the emeraldine form of polyaniline. Although the standard state reduction potentials of polyaniline and $\mathrm{ReO}_{4}^{-}$indicate that this reduction is possible, calculations employing the Nernst equation demonstrated that this is only energetically favourable when near-molar concentrations of the species involved are used. By comparison, oxidation of the leucoemeraldine form of polyaniline to emeraldine by $\mathrm{ReO}_{4}^{-}$ was shown by UV-visible spectrography to proceed readily. However due to the basic nature of NCS, the reduction potential of the emeraldine to pernigraniline transition can be altered, facilitating sorption of $\mathrm{ReO}_{4}^{-}$by the PANI-NCS composite through a redox process.

It was demonstrated that the PANI-NCS material readily sorbed $\mathrm{ReO}_{4}^{-}$from solutions conditions, having a measured maximum sorption capacity of $0.56 \pm 0.06 \mathrm{mmol} \mathrm{g}^{-1}$. The sorption was consistent with the Langmuir isotherm, the model conforming to the sorption data with an calculated $R^{2}$ value of 0.984 . 
XPS studies on the composite material post-sorption, demonstrated that the rhenium had undergone reduction from the $\mathrm{ReO}_{4}^{-}$species during the sorption process. The oxidation state of the rhenium could not be unequivocally determined by XPS, however, the +3 oxidation state is most likely. To account for the observed sorption capacity of the PANI films, it is suspected that most of the $\mathrm{ReO}_{4}^{-}$is converted to the $\mathrm{ReO}_{3}$ phase, with a surface layer of $\mathrm{Re}_{2} \mathrm{O}_{3}$ being observed by X-ray photoelectron spectroscopy. The existence of this surface layer indicates that reduction of $\mathrm{ReO}_{4}^{-}$by the composite material occurred. 


\section{Chapter 7}

\section{Summary of Thesis}

In this thesis the sorption properties of nanostructured calcium silicate (NCS) with respect to copper, phosphate, and perrhenate ions has been studied.

\subsection{Sorption of Copper}

It was demonstrated that NCS had a large affinity for the sorption of $\mathrm{Cu}^{2+}$ ions, the material being capable of sorbing up to $9 \pm 1 \mathrm{mmol} \mathrm{Cu}^{2+} \mathrm{g}^{-1} \mathrm{NCS}$, when $\mathrm{CuCl}_{2}$ was used as the source of copper. When sorption was undertaken from solutions of

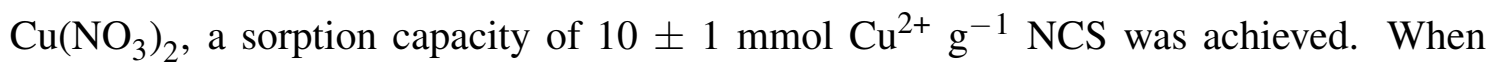
the amount of $\mathrm{Cu}^{2+}$ was limited, the copper was sorbed as an X-ray amorphous species, believed to be $\mathrm{Cu}(\mathrm{OH})_{2}$. If a significant excess of copper was present in the system, crystalline phases with a stoichiometry of $\mathrm{Cu}_{2} \mathrm{X}(\mathrm{OH})_{3}$ were observed by both electron microscopy and X-ray diffraction. In cases where $\mathrm{Cu}\left(\mathrm{NO}_{3}\right)_{2}$ was used as the copper source, the rouaite phase $\left(\mathrm{Cu}_{2}\left(\mathrm{NO}_{3}\right)(\mathrm{OH})_{3}\right)$ formed. When $\mathrm{CuCl}_{2}$ was used as the copper source, atacamite was the primary crystalline copper phase (56\%) formed, although clinoatacamite and paratacamite were also observed. These three phases share the $\mathrm{Cu}_{2} \mathrm{Cl}(\mathrm{OH})_{3}$ stoichiometry.

The presence of calcium is necessary for the NCS to sorb copper. Leaching calcium from the NCS with $\mathrm{HCl}$ prior to copper sorption resulted in a significant decrease in the maximum sorption capacity of the material. The calcium dependence on copper sorption was attributed to the exchange, in solution, of $\mathrm{Ca}^{2+}$ for $\mathrm{H}^{+}$, leading to an increase of the solution $\mathrm{pH}$ and, hence, a decrease in the solubility of the $\mathrm{Cu}^{2+}$ ion.

Under dynamic conditions, NCS applied at a loading of $1 \mathrm{~g} \mathrm{dm}^{-3}$ to a $1.6 \mathrm{mmol} \mathrm{dm}^{-3}$ $\mathrm{Cu}^{2+}$ solution would remove more than $99.5 \%$ of the copper from solution over a time period of 30-60 minutes. When the initial copper concentration was increased 


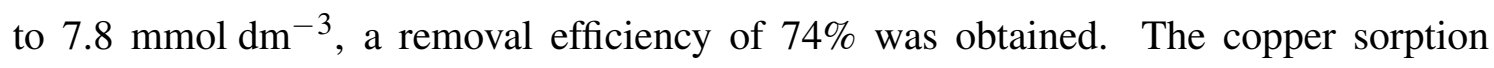
capacities noted above could only be attained when a significant excess of copper was present. Kinetic modelling of the rates of copper sorption and calcium desorption indicated that the sorption process was inconsistent with simple first- or second- order kinetics. Rather, the sorption kinetics are consistent with the system being rate limited by the diffusion of copper towards the NCS surface.

Overall, it has been shown that NCS would be an effective agent for the treatment of dilute copper waste solutions, achieving maximum sorption in a short time period.

\subsection{Magnetic Calcium Silicate Composites}

It was found that magnetite $\left(\mathrm{Fe}_{3} \mathrm{O}_{4}\right)$ could be incorporated into the NCS structure without significantly degrading its physical characteristics such as particle size distribution or surface area. This was achieved by dispersing magnetite with the sodium silicate precursor solution prior to the precipitation step during NCS synthesis. If a nanosized magnetite source was used (with a particle size of approximately $15 \mathrm{~nm}$ ), the magnetite was oxidised to the maghemite phase $\left(\gamma-\mathrm{Fe}_{2} \mathrm{O}_{3}\right)$ upon drying of the composite NCS material at $110^{\circ} \mathrm{C}$. The $\gamma-\mathrm{Fe}_{2} \mathrm{O}_{3}$ phase formed was shown to contain a diamagnetic, or antiferrimagnetic contaminant (most likely hematite) due to its lower than expected saturation magnetisation. If the magnetite source was bulk magnetite powder, the $\mathrm{Fe}_{3} \mathrm{O}_{4}$ phase was maintained during the drying stage. Up to 25 wt $\% \mathrm{Fe}_{3} \mathrm{O}_{4}$ could be incorporated into the composite with NCS while maintaining the desirable physical properties of the silicate, namely a high surface area.

It was shown that the magnetic phase was incorporated within the NCS material due to the absence of energy bands associated with iron from X-ray photoelectron spectra. This form of spectroscopy has a low sample penetration depth (a few hundreds of picometres), so is ideally suited for surface analysis.

The sorption capacity of the magnetic NCS composites, with respect to $\mathrm{Cu}^{2+}$, was comparable to that of the unmodified material. If a significant amount of magnetic 
material was present in the composite, the specific sorption capacity was suppressed insofar as the composite material contained less NCS on a per mass basis.

Attempts were made to utilise the magnetic nature of the $\gamma-\mathrm{Fe}_{2} \mathrm{O}_{3}$-NCS composites to aid separation of the material from solution following copper sorption. This was unsuccessful, however. It was found that the composite material degraded in the acidic $\mathrm{Cu}^{2+}$ solution, with the $\gamma-\mathrm{Fe}_{2} \mathrm{O}_{3}$ phase separating from the NCS. Due to this, it can be concluded that the inclusion of magnetic centres aids separation of the NCS from solution. However, the separation technique not been perfected and is not yet suitable for industrial application.

\subsection{Sorption of Phosphate by Magnetite-Calcium Silicate Composites}

The sorption of the phosphate ions, for example $\mathrm{H}_{2} \mathrm{PO}_{4}^{-}$, by $\mathrm{Fe}_{3} \mathrm{O}_{4}-\mathrm{NCS}$ composites prepared from bulk $\mathrm{Fe}_{3} \mathrm{O}_{4}$ was studied. The unmodified material was found to have a sorption capacity of $5.1 \pm 0.5 \mathrm{mmol} \mathrm{P} \mathrm{g}^{-1} \mathrm{NCS}$. The phosphate appears to be sorbed in the form of brushite $\left(\mathrm{CaHPO}_{4} \cdot 2 \mathrm{H}_{2} \mathrm{O}\right)$, although the $7.1 \mathrm{mmol} \mathrm{Ca}^{2+} \mathrm{g}^{-1}$ indicates that impurities of apatite could also be present. The $\mathrm{Fe}_{3} \mathrm{O}_{4}$ in the system did not contribute to the observed sorption capacity of the composite material. For example, a composite containing 25 wt $\% \mathrm{Fe}_{3} \mathrm{O}_{4}$ exhibits a sorption capacity of $3.9 \mathrm{mmol} \mathrm{P} \mathrm{g}^{-1}$ when modelled by the Langmuir isotherm, a sorption capacity approximately $75 \%$ that of the unmodified material. It was found that the sorption process conformed to the Langmuir isotherm at low sorbent loadings $\left(0.5 \mathrm{~g} \mathrm{dm}^{-3}\right)$, with a calculated correlation coefficient, $R^{2}$, for the isotherm model of 0.996 . As the sorbent loading was increased, the experimental data conformed less well to the Langmuir model. At a sorbent loading of $2 \mathrm{~g} \mathrm{dm}^{-3}$, the experimental data conformed more closely to the Tempkin isotherm than to the Langmuir one. This was attributed to the system not attaining a saturation sorption capacity at the higher sorbent loading.

The use of the $\mathrm{Fe}_{3} \mathrm{O}_{4}$-NCS composite as a sorbent in a continuous sorption system 
was demonstrated. It was shown that the $\mathrm{Fe}_{3} \mathrm{O}_{4}$-NCS composite could be utilised in a fluidised-bed type reactor. By using a conical reactor operating in upflow mode, it was shown that the settling rate of a $\mathrm{Fe}_{3} \mathrm{O}_{4}$-NCS composite at 25 wt $\% \mathrm{Fe}_{3} \mathrm{O}_{4}$ was approximately $0.6 \mathrm{~cm} \mathrm{~min}^{-1}$. The maximum sorption capacities attained by the composite in the continuous process were significantly lower than those in the batch experiments. This was attributed to the sorbent in the column either not reaching saturation (the phosphate effluent concentration remaining less than the influent concentration), or loss of some of the sorbent from the column. The sorbent loss was only noticed for a cylindrical column with a fluid velocity of $3.9 \mathrm{~cm} \mathrm{~min}^{-1}$. Therefore, it was concluded that the residence time of the solution in contact with the silicate is the limiting variable in terms of phosphate sorption. An industrial-scale application should be designed accordingly.

\subsection{Polyaniline Calcium Silicate Composites and Sorp- tion of Rhenium}

Composites of polyaniline and NCS were successfully prepared by the method of sorbing the polymer onto NCS from a solution of the polymer in 1-methylpyrrolidine. Composites containing up to $10 \mathrm{wt} \%$ PANI were easily prepared by this method. Spectrographic analysis showed the polymer to be bound to the NCS as the emeraldine base form. Shifts in the visible absorption bands suggested PANI was bound to the NCS via hydrogen bonding between the amine hydrogen of the polymer and oxygen present within the NCS structure.

Both polyaniline films and the PANI-NCS composite were used to sorb $\mathrm{ReO}_{4}^{-}$from solution. It was demonstrated that PANI films in the leucoemeraldine base form will sorb $\mathrm{ReO}_{4}^{-}$by a redox mechanism. The emeraldine forms of PANI films, by comparison, do not undergo redox reactions with $\mathrm{ReO}_{4}^{-}$. However, it was found that when $\mathrm{ReO}_{4}^{-}$ sorption was undertaken by the PANI-NCS composite material, that some of the $\mathrm{ReO}_{4}^{-}$ was reduced to a rhenium oxide species in the +3 oxidation state. This was shown by XPS analysis. The exact nature of the rhenium species formed could not be unequivocally 
determined. The species responsible for the reduction of $\mathrm{ReO}_{4}^{-}$was also indeterminable through XPS analysis. Expected changes in the binding energy of the $\mathrm{N} 1 \mathrm{~s}$ electrons should the polymer be oxidised to its pernigraniline form, for example, were not present.

The sorption capacity of the PANI-NCS material towards $\mathrm{ReO}_{4}^{-}$was determined to be $0.58 \pm 0.05 \mathrm{mmol} \mathrm{Re}^{-1}$, with the sorption conforming to a Langmuir isotherm, having a $R^{2}$ value of 0.984 . It is suspected $\mathrm{ReO}_{4}^{-}$is reduced to the thermodynamically favoured $\mathrm{ReO}_{3}$ phase, with a surface layer of $\mathrm{Re}_{2} \mathrm{O}_{3}$ being observed during XPS measurements. Thus, the PANI-NCS composites are suitable sorbent materials for $\mathrm{ReO}_{4}^{-}$.

\subsection{Future Work}

As a result of the work presented herein, the use of NCS as a sorbent material for copper is now well characterised, as is use of the magnetic composites of NCS for copper or phosphate sorption. Utilisation of the magnetic properties of superparamagnetic $\gamma-\mathrm{Fe}_{2} \mathrm{O}_{3}$ NCS materials to aid their separation from copper solutions was unsuccessful, as the $\gamma-\mathrm{Fe}_{2} \mathrm{O}_{3}$ separated from the NCS material in the acidic copper solution. The sorption characteristics, with respect to copper, of magnetic composites prepared with bulk $\mathrm{Fe}_{3} \mathrm{O}_{4}$ were briefly investigated and were found to be comparable to those of the unmodified NCS. Use of magnetic techniques to separate these materials following copper sorption was not investigated and should be studied in a future project.

Characterisation of the sorption properties of the $\mathrm{Fe}_{3} \mathrm{O}_{4}$-NCS materials with respect to phosphate demonstrated that the materials exhibited a high affinity for the ion. It was shown that the materials were suitable for use in a continuous sorption system, with the magnetic nature of the composite material causing flocculation. The continuous sorption system was only tested at the laboratory scale. Testing the materials on larger scale systems would be a logical progression of this research.

The preparation of PANI-NCS composites provided a material which acted as a substrate for the sorption and partial reduction of $\mathrm{ReO}_{4}^{-}$. Although the concurrent oxidation of the PANI could not be confirmed, it is likely that the polyaniline retains its redox activity. 
This leads to three possible avenues of research: The first is to definitively characterise the redox properties of the PANI-NCS composite, as a better understanding of the material's electrochemical properties would be desirable. The second area of research would be to characterise the sorption properties of the PANI-NCS composite with respect to $\mathrm{TcO}_{4}^{-}$, as perrhenate was chosen as a target sorbate due to its chemical similarities to pertechnetate. Finally, the a study focusing on the recovery of $\mathrm{ReO}_{4}^{-}$in its own right may prove interesting. A comparison should be made of the sorption properties of perrhenate and a typical source contaminant, such as molybdate. 


\section{Appendix A}

\section{Data for Figures}

Data from which graphs were constructed is included below, unless it is already mentioned in the main body of the text.

Data for Figure 3.4

Leaching of $\mathrm{Ca}^{2+}$ from NCS.

\begin{tabular}{llr}
\hline Time/ min & {$\left[\mathrm{Ca}^{2+}\right] / \mathbf{~ m m o l ~} \mathbf{d m}^{-\mathbf{3}}$} & $\mathbf{p H}$ \\
\hline 0 & $0.010 \pm 0.001$ & 7.18 \\
1 & $0.303 \pm 0.003$ & 9.43 \\
3 & $0.516 \pm 0.005$ & 9.69 \\
6 & $0.621 \pm 0.005$ & 9.70 \\
10 & $0.704 \pm 0.008$ & 9.82 \\
15 & $0.776 \pm 0.008$ & 9.96 \\
30 & $0.913 \pm 0.004$ & 10.06 \\
60 & $1.10 \pm 0.01$ & 10.12 \\
240 & $1.34 \pm 0.01$ & 10.19 \\
480 & $1.44 \pm 0.01$ & 10.16 \\
1441 & $1.33 \pm 0.02$ & 10.01 \\
\hline
\end{tabular}


Data for Figure 3.5

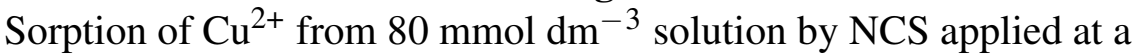
loading of $1 \mathrm{~g} \mathrm{dm}^{-3}$.

\begin{tabular}{lccc}
\hline Time/ min & {$\left[\mathrm{Cu}^{2+}\right] / \mathbf{~ m m o l ~} \mathbf{d m}^{-\mathbf{3}}$} & {$\left[\mathrm{Ca}^{2+}\right] / \mathbf{~ m m o l ~} \mathbf{d m}^{-\mathbf{3}}$} & $\mathbf{p H}$ \\
\hline \multicolumn{4}{c}{ Figure 3.5(a), $\mathbf{C u C l}_{\mathbf{2}}$} \\
\hline 0 & $77.3 \pm 0.5$ & $-0.2 \pm 0.1$ & \\
1 & $74.0 \pm 0.3$ & $2.5 \pm 0.3$ & 3.12 \\
3 & $72.2 \pm 0.1$ & $4.3 \pm 0.1$ & 3.87 \\
5 & $70.8 \pm 0.7$ & $5.1 \pm 0.3$ & 3.87 \\
10 & $69.4 \pm 0.7$ & $5.4 \pm 0.1$ & 3.89 \\
15 & $69.4 \pm 0.7$ & $5.9 \pm 0.2$ & 3.75 \\
30 & $68.1 \pm 0.1$ & $6.3 \pm 0.4$ & 3.92 \\
60 & $67.2 \pm 0.6$ & $6.5 \pm 0.2$ & 4.01 \\
240 & $67.3 \pm 0.1$ & $6.3 \pm 0.1$ & 4.10 \\
478 & $67.2 \pm 0.3$ & $6.2 \pm 0.1$ & 4.05 \\
1440 & $67.5 \pm 0.5$ & $6.5 \pm 0.2$ & 4.02 \\
\hline & \multicolumn{4}{c}{ Figure 3.5(b), Cu(NO $\mathbf{3}_{\mathbf{2}}$} & \\
\hline 0 & $81.8 \pm 0.6$ & $-0.07 \pm 0.03$ & 4.13 \\
1 & $79.5 \pm 0.3$ & $1.67 \pm 0.01$ & 4.40 \\
3 & $79.3 \pm 0.1$ & $1.99 \pm 0.01$ & 4.41 \\
5 & $79.0 \pm 0.3$ & $2.13 \pm 0.01$ & 4.42 \\
10 & $77.6 \pm 0.1$ & $2.38 \pm 0.01$ & 4.42 \\
15 & $78.0 \pm 0.3$ & $2.48 \pm 0.03$ & 4.40 \\
30 & $76.5 \pm 1.0$ & $2.94 \pm 0.02$ & 4.39 \\
60 & $75.5 \pm 0.5$ & $5.78 \pm 0.01$ & 4.41 \\
249 & $73.3 \pm 0.6$ & $5.72 \pm 0.02$ & 4.43 \\
477 & $74.3 \pm 0.5$ & $5.59 \pm 0.07$ & 4.45 \\
1440 & $72.4 \pm 0.4$ & $5.64 \pm 0.04$ & 4.58 \\
\hline & & &
\end{tabular}


Data for Figure 3.6

Sorption of $\mathrm{Cu}^{2+}$ from low concentration solution by NCS.

\begin{tabular}{lccc}
\hline Time/ min & {$\left[\mathrm{Cu}^{2+}\right] / \mathbf{~ m m o l ~ d m}{ }^{-3}$} & {$\left[\mathrm{Ca}^{2+}\right] / \mathbf{~ m m o l ~} \mathbf{d m}^{-\mathbf{3}}$} & $\mathbf{p H}$ \\
\hline \multicolumn{4}{c}{ Figure 3.6(a), $\mathbf{C u}\left(\mathbf{N O}_{3}\right)_{\mathbf{2}}$} \\
\hline 0 & $7.8 \pm 0.2$ & $-0.07 \pm 0.02$ & \\
1 & $7.2 \pm 0.1$ & $1.42 \pm 0.07$ & 5.74 \\
3 & $6.9 \pm 0.1$ & $1.84 \pm 0.04$ & 5.70 \\
5 & $6.7 \pm 0.1$ & $1.93 \pm 0.02$ & 5.74 \\
10 & $6.39 \pm 0.03$ & $2.06 \pm 0.04$ & 5.76 \\
15 & $6.32 \pm 0.05$ & $2.3 \pm 0.1$ & 5.78 \\
30 & $6.08 \pm 0.05$ & $2.4 \pm 0.1$ & 5.80 \\
80 & $5.69 \pm 0.04$ & $2.7 \pm 0.1$ & 5.81 \\
240 & $4.80 \pm 0.06$ & $3.9 \pm 0.1$ & 5.86 \\
480 & $4.03 \pm 0.05$ & $4.54 \pm 0.04$ & 5.89 \\
1440 & $2.04 \pm 0.03$ & $6.24 \pm 0.06$ & 6.05 \\
\hline
\end{tabular}

\begin{tabular}{lccc}
\hline \multicolumn{4}{c}{ Figure 3.6(b), $\mathbf{C u}\left(\mathbf{N O}_{\mathbf{3}} \mathbf{~} \mathbf{2}\right.$} \\
\hline 0 & $1.6 \pm 0.1$ & $0.005 \pm 0.003$ & 5.38 \\
1 & $0.85 \pm 0.01$ & $0.966 \pm 0.007$ & 6.00 \\
3 & $0.63 \pm 0.02$ & $1.320 \pm 0.005$ & 6.08 \\
5 & $0.47 \pm 0.09$ & $1.455 \pm 0.004$ & 6.23 \\
10 & $0.327 \pm 0.005$ & $1.704 \pm 0.005$ & 6.37 \\
15 & $0.172 \pm 0.002$ & $1.91 \pm 0.02$ & 7.01 \\
30 & $0.02 \pm 0.02$ & $2.20 \pm 0.03$ & 7.31 \\
60 & $0.00 \pm 0.07$ & $2.338 \pm 0.005$ & 7.72 \\
240 & $-0.02 \pm 0.01$ & $2.45 \pm 0.01$ & 8.10 \\
480 & $-0.009 \pm 0.008$ & $2.50 \pm 0.01$ & 8.33 \\
1440 & $-0.012 \pm 0.005$ & $2.532 \pm 0.008$ & 9.10 \\
\hline & Figure 3.6(c), CuCl & \\
\hline 0 & $1.31 \pm 0.02$ & $0.005 \pm 0.001$ & 4.73 \\
1 & $0.77 \pm 0.02$ & $0.946 \pm 0.003$ & 6.17 \\
3 & $0.48 \pm 0.02$ & $1.220 \pm 0.004$ & 6.34 \\
5 & $0.38 \pm 0.04$ & $1.41 \pm 0.01$ & 6.57 \\
10 & $0.130 \pm 0.002$ & $1.64 \pm 0.02$ & 6.77 \\
15 & $0.065 \pm 0.003$ & $1.78 \pm 0.02$ & 6.96 \\
30 & $0.01 \pm 0.01$ & $1.97 \pm 0.01$ & 7.73 \\
60 & $0.03 \pm 0.01$ & $2.03 \pm 0.02$ & 8.31 \\
240 & $0.03 \pm 0.03$ & $2.183 \pm 0.004$ & 9.21 \\
480 & $0.02 \pm 0.02$ & $2.17 \pm 0.01$ & 9.29 \\
1440 & $0.02 \pm 0.02$ & $2.17 \pm 0.01$ & 9.27 \\
\hline & & &
\end{tabular}


Data for Figure 3.7

Sorption of $\mathrm{Cu}^{2+}$ by $\mathrm{pH}$ adjusted NCS.

\begin{tabular}{|c|c|c|c|}
\hline Time/ min & {$\left[\mathrm{Cu}^{2+}\right] / \mathrm{mmol}_{\mathbf{d m}}^{-3}$} & {$\left[\mathrm{Ca}^{2+}\right] / \mathrm{mmol} \mathbf{d m}^{-3}$} & pH \\
\hline \multicolumn{4}{|c|}{ Figure 3.7(a), $\mathrm{Cu}\left(\mathrm{NO}_{3}\right)_{2}$} \\
\hline 0 & $80.7 \pm 0.7$ & $-0.06 \pm 0.04$ & 4.03 \\
\hline 1 & $80.4 \pm 0.7$ & $0.112 \pm 0.002$ & 4.50 \\
\hline 3 & $79.6 \pm 0.7$ & $0.184 \pm 0.001$ & 4.52 \\
\hline 5 & $80.6 \pm 0.5$ & $0.225 \pm 0.001$ & 4.51 \\
\hline 10 & $80.6 \pm 0.6$ & $0.308 \pm 0.004$ & 4.44 \\
\hline 15 & $81.0 \pm 0.2$ & $0.350 \pm 0.003$ & 4.41 \\
\hline 30 & $80.7 \pm 0.1$ & $0.436 \pm 0.003$ & 4.40 \\
\hline 60 & $81.0 \pm 0.9$ & $0.546 \pm 0.003$ & 4.40 \\
\hline 240 & $79.5 \pm 0.8$ & $1.11 \pm 0.01$ & 4.44 \\
\hline 480 & $78.8 \pm 0.3$ & $1.293 \pm 0.006$ & 4.50 \\
\hline 1440 & $78.5 \pm 0.3$ & $1.374 \pm 0.004$ & 4.52 \\
\hline \multicolumn{4}{|c|}{ Figure 3.7(b), $\mathrm{Cu}\left(\mathrm{NO}_{3}\right)_{2}$} \\
\hline 0 & $1.51 \pm 0.02$ & $0.02 \pm 0.01$ & 5.30 \\
\hline 1 & $1.49 \pm 0.02$ & $0.12 \pm 0.01$ & 5.74 \\
\hline 3 & $1.46 \pm 0.02$ & $0.166 \pm 0.003$ & 5.88 \\
\hline 5 & $1.40 \pm 0.01$ & $0.188 \pm 0.003$ & 5.94 \\
\hline 10 & $1.336 \pm 0.003$ & $0.26 \pm 0.01$ & 6.02 \\
\hline 15 & $1.38 \pm 0.01$ & $0.29 \pm 0.01$ & 6.02 \\
\hline 30 & $1.30 \pm 0.01$ & $0.36 \pm 0.01$ & 6.09 \\
\hline 60 & $1.22 \pm 0.02$ & $0.45 \pm 0.02$ & 6.12 \\
\hline \multicolumn{4}{|c|}{ Figure 3.7(c), $\mathrm{CuCl}_{2}$} \\
\hline 0 & $1.49 \pm 0.01$ & $0.01 \pm 0.01$ & 5.56 \\
\hline 1 & $1.45 \pm 0.02$ & $0.13 \pm 0.01$ & 5.87 \\
\hline 3 & $1.34 \pm 0.02$ & $0.18 \pm 0.01$ & 5.99 \\
\hline 5 & $1.37 \pm 0.02$ & $0.21 \pm 0.01$ & 6.01 \\
\hline 10 & $1.34 \pm 0.03$ & $0.271 \pm 0.004$ & 6.07 \\
\hline 15 & $1.33 \pm 0.02$ & $0.32 \pm 0.01$ & 6.11 \\
\hline 30 & $1.21 \pm 0.03$ & $0.42 \pm 0.01$ & 6.15 \\
\hline 61 & $1.11 \pm 0.01$ & $0.539 \pm 0.003$ & 6.17 \\
\hline
\end{tabular}

Data for Figure 3.8

Sorption of $\mathrm{Cu}^{2+}$ in the presence of $\mathrm{I}_{3}^{-}$by NCS.

\begin{tabular}{|c|c|c|c|c|}
\hline Time/ min & {$\left[\mathrm{Cu}^{2+}\right] / \mathbf{m m o l} \mathbf{d m}^{-3}$} & {$\left[\mathrm{Ca}^{2+}\right] / \mathbf{m m o l} \mathbf{d m}^{-3}$} & {$\left[\mathrm{I}_{3}^{-}\right] / \mathbf{m m o l ~ d m}^{-3}$} & $\overline{\text { pH }}$ \\
\hline 0 & $1.42 \pm 0.01$ & $-0.02 \pm 0.01$ & $0.14 \pm 0.01$ & 4.19 \\
\hline 1 & $0.79 \pm 0.01$ & $1.21 \pm 0.02$ & $0.04 \pm 0.01$ & 4.61 \\
\hline 3 & $0.54 \pm 0.02$ & $1.49 \pm 0.01$ & $0.04 \pm 0.01$ & 4.66 \\
\hline 5 & $0.43 \pm 0.01$ & $1.66 \pm 0.01$ & $0.04 \pm 0.01$ & 4.66 \\
\hline 10 & $0.18 \pm 0.01$ & $1.88 \pm 0.02$ & $0.04 \pm 0.01$ & 4.74 \\
\hline 15 & $0.06 \pm 0.01$ & $2.03 \pm 0.02$ & $0.03 \pm 0.01$ & 4.87 \\
\hline 30 & $-0.03 \pm 0.01$ & $2.26 \pm 0.01$ & $0.03 \pm 0.01$ & 5.17 \\
\hline 60 & $-0.05 \pm 0.02$ & $2.44 \pm 0.02$ & $0.03 \pm 0.01$ & 6.15 \\
\hline
\end{tabular}


Data for Figure 4.5(b)

Particle size distribution of superparamagnetic $\mathrm{Fe}_{3} \mathrm{O}_{4}$-NCS composites.

\begin{tabular}{lccr}
\hline \multirow{2}{*}{ wt $\% \mathbf{F e}_{\mathbf{3}} \mathbf{O}_{\mathbf{4}}$} & \multicolumn{3}{c}{ Size/ $\mu \mathrm{m}$} \\
\cline { 2 - 4 } & \multicolumn{3}{c}{ Percentile } \\
\cline { 2 - 4 } & $\mathbf{1 0}^{\text {th }}$ & $\mathbf{5 0}^{\text {th }}$ & $\mathbf{9 0}^{\text {th }}$ \\
\hline 0.00 & 2.16 & 3.37 & 9.76 \\
1.51 & 2.19 & 3.61 & 11.37 \\
3.01 & 1.84 & 2.98 & 8.76 \\
4.49 & 1.68 & 2.62 & 6.76 \\
7.41 & 1.36 & 2.12 & 5.28 \\
10.27 & 1.34 & 2.04 & 4.81 \\
14.46 & 1.35 & 2.10 & 4.85 \\
100 & 0.30 & 0.43 & 1.57 \\
\hline
\end{tabular}

Data for Figure 4.6

Surface area of $\gamma-\mathrm{Fe}_{2} \mathrm{O}_{3}$-NCS composites.

\begin{tabular}{lrr}
\hline wt $\%$ & $\gamma-\mathrm{Fe}_{2} \mathbf{O}_{3}$ & \multicolumn{2}{c}{ Surface area/ $\mathbf{~}^{\mathbf{2}} \mathbf{g}^{-\mathbf{1}}$} \\
\cline { 2 - 3 } & 2-ethoxyethanol & water \\
\hline 0.00 & 437 & 126 \\
2.05 & 664 & 157 \\
4.08 & 565 & 160 \\
6.08 & 538 & 156 \\
10.03 & 561 & 157 \\
13.89 & 538 & 150 \\
19.52 & 444 & 139 \\
\hline
\end{tabular}

Data for Figures 4.10-4.11

Magnetic properties of $\gamma-\mathrm{Fe}_{2} \mathrm{O}_{3}$-NCS composites.

\begin{tabular}{|c|c|c|c|c|}
\hline \multirow{2}{*}{ wt $\% \gamma-\mathrm{Fe}_{2} \mathrm{O}_{3}$} & \multicolumn{2}{|c|}{ As made } & \multicolumn{2}{|c|}{ After $\mathrm{Cu}^{2+}$ sorption } \\
\hline & $\sigma_{s}^{a}$ & $\sigma_{r}$ & $\sigma_{s}$ & $\sigma_{r}$ \\
\hline 0 & - & -0.011 & - & - \\
\hline 2.05 & 0.06 & 0.004 & 0.56 & 0.002 \\
\hline 4.08 & 1.15 & 0.004 & 1.15 & 0.005 \\
\hline 6.08 & 2.24 & 0.013 & 1.72 & 0.007 \\
\hline 10.03 & 3.52 & 0.018 & 3.26 & 0.012 \\
\hline 13.89 & 4.21 & 0.016 & 4.13 & 0.017 \\
\hline 19.52 & 6.38 & 0.031 & - & - \\
\hline
\end{tabular}

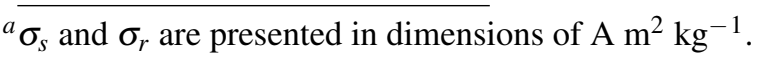


Data for Figure 4.15(b)

Particle size distribution of bulk $\mathrm{Fe}_{3} \mathrm{O}_{4}$-NCS composites.

\begin{tabular}{llrr}
\hline \multirow{2}{*}{ wt $\% \mathbf{F e}_{\mathbf{3}} \mathbf{O}_{\mathbf{4}}$} & \multicolumn{3}{c}{ Size/ $\mu \mathrm{m}$} \\
\cline { 2 - 4 } & \multicolumn{3}{c}{ Percentile } \\
\cline { 2 - 4 } & $\mathbf{1 0}^{\text {th }}$ & $\mathbf{5 0}^{\text {th }}$ & $\mathbf{9 0}^{\text {th }}$ \\
\hline 0 & 1.23 & 2.35 & 8.66 \\
1 & 2.04 & 3.91 & 10.12 \\
5 & 1.92 & 3.58 & 9.45 \\
10 & 1.61 & 2.97 & 8.99 \\
15 & 0.71 & 0.95 & 4.14 \\
20 & 0.71 & 0.97 & 4.03 \\
25 & 0.71 & 1.03 & 3.76 \\
100 & 0.22 & 0.29 & 0.71 \\
\hline
\end{tabular}

Data for Figure 4.18

Sorption of $\mathrm{Cu}^{2+}$ by $\gamma-\mathrm{Fe}_{2} \mathrm{O}_{3}$-NCS composites.

Time/ min $\left[\mathrm{Cu}^{2+}\right] / \mathbf{m m o l} \mathbf{d m}^{-3} \quad\left[\mathrm{Ca}^{2+}\right] / \mathbf{m m o l} \mathbf{d m}^{-\mathbf{3}} \quad \mathbf{p H}$

Figure 4.18(a)

\begin{tabular}{lcrr}
\hline 0 & $82.8 \pm 0.1$ & $-0.042 \pm 0.004$ & 4.12 \\
1 & $81 \pm 1$ & $1.50 \pm 0.02$ & 4.35 \\
3 & $79 \pm 1$ & $1.76 \pm 0.02$ & 4.38 \\
5 & $79 \pm 1$ & $1.87 \pm 0.01$ & 4.40 \\
10 & $79.0 \pm 0.7$ & $2.05 \pm 0.02$ & 4.4 \\
15 & $78.0 \pm 0.6$ & $2.20 \pm 0.02$ & 4.37 \\
30 & $77 \pm 1$ & $2.57 \pm 0.01$ & 4.42 \\
60 & $75 \pm 1$ & $4.553 \pm 0.001$ & 4.43 \\
240 & $73.0 \pm 0.5$ & $5.30 \pm 0.02$ & 4.38 \\
480 & $72.7 \pm 0.8$ & $5.30 \pm 0.06$ & 4.48 \\
1440 & $71.8 \pm 0.7$ & $5.46 \pm 0.04$ & 4.50 \\
\hline \multicolumn{4}{c}{ Figure 4.18(b) } \\
\hline 0 & $1.62 \pm 0.05$ & $-0.026 \pm 0.002$ & 4.38 \\
1 & $0.406 \pm 0.005$ & $1.296 \pm 0.002$ & 5.90 \\
3 & $0.107 \pm 0.001$ & $1.612 \pm 0.003$ & 6.37 \\
5 & $0.023 \pm 0.006$ & $1.768 \pm 0.002$ & 6.73 \\
10 & $0.005 \pm 0.004$ & $1.917 \pm 0.005$ & 7.14 \\
15 & - & $1.981 \pm 0.007$ & 7.08 \\
30 & $-0.003 \pm 0.001$ & $2.076 \pm 0.009$ & 7.60 \\
60 & $-0.003 \pm 0.005$ & $2.087 \pm 0.006$ & 7.77 \\
\hline
\end{tabular}


Data for Figure 4.19

Isotherm sorption of $\mathrm{Cu}^{2+}$ by $\mathrm{Fe}_{3} \mathrm{O}_{4}$-NCS composite at 25 wt $\% \mathrm{Fe}_{3} \mathrm{O}_{4}$.

\begin{tabular}{cc}
\hline $\mathbf{C u}^{2+}$ uptake/ mmol g & {$\left[\mathrm{Cu}^{2+}\right]_{\mathbf{E q}} / \mathbf{~ m m o l ~} \mathbf{~ m}^{-\mathbf{3}}$} \\
\hline \multicolumn{2}{c}{$\mathbf{N C S}$} \\
\hline $0.0004 \pm 0.0003$ & $0.0002 \pm 0.0001$ \\
$1.73 \pm 0.06$ & $0.0019 \pm 0.0003$ \\
$3.6 \pm 0.1$ & $0.003 \pm 0.001$ \\
$5.64 \pm 0.2$ & $0.006 \pm 0.001$ \\
$7.3 \pm 0.3$ & $0.43 \pm 0.03$ \\
$8.5 \pm 0.4$ & $0.82 \pm 0.04$ \\
$8.6 \pm 0.5$ & $1.68 \pm 0.07$ \\
$9.0 \pm 0.6$ & $2.3 \pm 0.1$ \\
$8.5 \pm 0.9$ & $4.0 \pm 0.2$ \\
$9.1 \pm 0.8$ & $4.4 \pm 0.2$ \\
$9.9 \pm 0.8$ & $4.4 \pm 0.2$
\end{tabular}

$\mathrm{Fe}_{3} \mathrm{O}_{4}$-NCS composite

\begin{tabular}{cc}
\hline $0.001 \pm 0.004$ & $0.000 \pm 0.002$ \\
$1.72 \pm 0.09$ & $0.05 \pm 0.01$ \\
$3.4 \pm 0.1$ & $0.016 \pm 0.001$ \\
$4.6 \pm 0.3$ & $0.51 \pm 0.07$ \\
$5.3 \pm 0.5$ & $0.98 \pm 0.1$ \\
$5.7 \pm 0.7$ & $1.49 \pm 0.3$ \\
$7.1 \pm 0.8$ & $2.13 \pm 0.2$ \\
$7.4 \pm 0.9$ & $2.35 \pm 0.4$ \\
$7.6 \pm 1$ & $3.43 \pm 0.5$ \\
$8.2 \pm 2$ & $4.82 \pm 2$ \\
$8.5 \pm 1$ & $5.04 \pm 0.5$ \\
\hline
\end{tabular}

Data for Figure 5.1

Phosphate uptake for $\mathrm{Fe}_{3} \mathrm{O}_{4}$-NCS composites.

\begin{tabular}{ll}
\hline wt $\% \mathrm{Fe}_{\mathbf{3}} \mathbf{O}_{\mathbf{4}}$ & Phosphate uptake/ $\mathbf{~ m m o l ~ ~ ^ { - 1 }}$ \\
\hline 0 & $5.4 \pm 0.3$ \\
1 & $5.5 \pm 0.4$ \\
5 & $4.8 \pm 0.3$ \\
10 & $4.3 \pm 0.4$ \\
15 & $4.2 \pm 0.3$ \\
20 & $4.2 \pm 0.3$ \\
25 & $3.9 \pm 0.4$ \\
100 & $0.3 \pm 0.2$ \\
\hline
\end{tabular}


Data for Figure 5.2

Isotherm sorption of phosphate by $\mathrm{Fe}_{3} \mathrm{O}_{4}$-NCS composite at 25 wt $\% \mathrm{Fe}_{3} \mathrm{O}_{4}$.

\begin{tabular}{cc}
\hline Phosphate uptake/ mmol g & {$\left[\mathrm{H}_{\mathrm{x}} \mathrm{PO}_{4}^{(3-\mathrm{x})-}\right]_{\mathbf{E q}} / \mathbf{~ m m o l ~ d m}^{-\mathbf{3}}$} \\
\hline & $\mathbf{0 . 5} \mathbf{~ g ~ d m}^{-\mathbf{3}}$ loading \\
\hline $0.0001 \pm 0.0003$ & $-0.0001 \pm 0.0002$ \\
$0.0151 \pm 0.0008$ & $0.0008 \pm 0.0002$ \\
$0.086 \pm 0.003$ & $0.0002 \pm 0.0002$ \\
$0.177 \pm 0.006$ & $-0.0001 \pm 0.0002$ \\
$0.83 \pm 0.03$ & $0.0042 \pm 0.0002$ \\
$1.65 \pm 0.06$ & $0.0155 \pm 0.0005$ \\
$3.84 \pm 0.2$ & $2.82 \pm 0.02$ \\
$3.89 \pm 0.3$ & $7.64 \pm 0.05$ \\
\hline & $\mathbf{1 . 0} \mathbf{g ~ d m}^{-\mathbf{3}}$ loading \\
\hline $0.0000 \pm 0.0002$ & $0.0000 \pm 0.0002$ \\
$0.0100 \pm 0.0004$ & $0.0000 \pm 0.0002$ \\
$0.049 \pm 0.001$ & $-0.0003 \pm 0.0002$ \\
$0.100 \pm 0.002$ & $0.0001 \pm 0.0002$ \\
$0.49 \pm 0.01$ & $0.0008 \pm 0.0002$ \\
$0.97 \pm 0.02$ & $0.0022 \pm 0.0006$ \\
$3.25 \pm 0.1$ & $1.58 \pm 0.02$ \\
$3.62 \pm 0.2$ & $6.22 \pm 0.06$ \\
\hline
\end{tabular}

Data for Figure 5.3

Sorption of phosphate by $\mathrm{Fe}_{3} \mathrm{O}_{4}$-NCS composite.

\begin{tabular}{llr}
\hline Time/ min & {$\left[\mathrm{H}_{\mathrm{x}} \mathrm{PO}_{4}^{(3-\mathrm{x})-}\right] / \mathbf{~ m m o l ~ d m}^{-\mathbf{3}}$} & $\mathbf{p H}$ \\
\hline 0 & $0.99 \pm 0.01$ & 5.39 \\
5 & $0.32 \pm 0.01$ & 9.97 \\
10 & $0.27 \pm 0.01$ & 9.96 \\
15 & $0.24 \pm 0.01$ & 10.00 \\
20 & $0.21 \pm 0.01$ & 9.99 \\
30 & $0.19 \pm 0.01$ & 10.11 \\
45 & $0.16 \pm 0.01$ & 10.11 \\
60 & $0.14 \pm 0.01$ & 10.18 \\
\hline
\end{tabular}


Data for Figure 5.4

Continuous uptake of phosphate by $\mathrm{Fe}_{3} \mathrm{O}_{4}$-NCS.

\begin{tabular}{|c|c|c|}
\hline Reactor Volumes & {$\left[\mathrm{H}_{\mathrm{x}} \mathrm{PO}_{4}^{(3-\mathrm{x})-}\right] / \mathbf{m m o l} \mathbf{d m}^{-\mathbf{3}}$} & $\mathbf{p H}$ \\
\hline \multicolumn{3}{|c|}{ Figure 5.4(a) } \\
\hline $0.15 \pm 0.02$ & $0.001 \pm 0.003$ & 10.90 \\
\hline $0.70 \pm 0.02$ & $0.08 \pm 0.02$ & 10.16 \\
\hline $1.39 \pm 0.02$ & $0.37 \pm 0.02$ & 9.59 \\
\hline $2.07 \pm 0.02$ & $0.65 \pm 0.02$ & 8.96 \\
\hline $2.76 \pm 0.02$ & $0.72 \pm 0.02$ & 8.37 \\
\hline $3.45 \pm 0.02$ & $0.76 \pm 0.02$ & 7.72 \\
\hline $4.13 \pm 0.02$ & $0.86 \pm 0.03$ & 7.68 \\
\hline $4.82 \pm 0.02$ & $0.84 \pm 0.03$ & 7.52 \\
\hline $5.50 \pm 0.02$ & $0.86 \pm 0.03$ & 7.48 \\
\hline $6.19 \pm 0.02$ & $0.87 \pm 0.03$ & 7.35 \\
\hline $6.88 \pm 0.02$ & $0.90 \pm 0.03$ & 7.29 \\
\hline \multicolumn{3}{|c|}{ Figure 5.4(b) } \\
\hline $0.15 \pm 0.02$ & $0.001 \pm 0.003$ & 10.90 \\
\hline $0.70 \pm 0.02$ & $0.08 \pm 0.02$ & 10.16 \\
\hline $1.39 \pm 0.02$ & $0.37 \pm 0.02$ & 9.59 \\
\hline $2.07 \pm 0.02$ & $0.65 \pm 0.02$ & 8.96 \\
\hline $2.76 \pm 0.02$ & $0.72 \pm 0.02$ & 8.37 \\
\hline $3.45 \pm 0.02$ & $0.76 \pm 0.02$ & 7.72 \\
\hline $4.13 \pm 0.02$ & $0.86 \pm 0.03$ & 7.68 \\
\hline $4.82 \pm 0.02$ & $0.84 \pm 0.03$ & 7.52 \\
\hline $5.50 \pm 0.02$ & $0.86 \pm 0.03$ & 7.48 \\
\hline $6.19 \pm 0.02$ & $0.87 \pm 0.03$ & 7.35 \\
\hline $6.88 \pm 0.02$ & $0.90 \pm 0.03$ & 7.29 \\
\hline \multicolumn{3}{|c|}{ Figure 5.4(c) } \\
\hline $2.23 \pm 0.25$ & $0.107 \pm 0.001$ & 10.54 \\
\hline $4.22 \pm 0.25$ & $0.429 \pm 0.003$ & 9.70 \\
\hline $6.20 \pm 0.25$ & $0.553 \pm 0.003$ & 9.28 \\
\hline $8.18 \pm 0.25$ & $0.605 \pm 0.003$ & 9.06 \\
\hline $10.17 \pm 0.25$ & $0.646 \pm 0.003$ & 8.80 \\
\hline $12.15 \pm 0.25$ & $0.687 \pm 0.003$ & 8.72 \\
\hline $14.14 \pm 0.25$ & $0.750 \pm 0.003$ & 8.23 \\
\hline $16.12 \pm 0.25$ & $0.810 \pm 0.006$ & 7.91 \\
\hline $18.10 \pm 0.25$ & $0.795 \pm 0.006$ & 8.12 \\
\hline $20.09 \pm 0.25$ & $0.889 \pm 0.006$ & 7.64 \\
\hline $22.07 \pm 0.25$ & $0.974 \pm 0.006$ & 7.33 \\
\hline $24.06 \pm 0.25$ & $1.006 \pm 0.006$ & 7.33 \\
\hline $26.04 \pm 0.25$ & $1.059 \pm 0.006$ & 7.26 \\
\hline $28.02 \pm 0.25$ & $1.075 \pm 0.006$ & 7.03 \\
\hline $30.01 \pm 0.25$ & $1.056 \pm 0.006$ & 6.86 \\
\hline
\end{tabular}


Data for Figures 6.22-6.23

Isotherm sorption of $\mathrm{ReO}_{4}^{-}$by PANI-NCS composite.

\begin{tabular}{|c|c|c|c|}
\hline Rhenium uptake/ mmol $\mathrm{g}^{-1}$ & {$\left[\mathrm{ReO}_{4}^{-}\right]_{\mathbf{E q}} / \mathbf{m m o l} \mathbf{d m}^{-\mathbf{3}}$} & {$\left[\mathrm{Ca}^{2+}\right]_{\mathbf{E q}} / \mathbf{m m o l} \mathbf{d m}^{-3}$} & pH \\
\hline $0.004 \pm 0.004$ & $0.003 \pm 0.003$ & $0.790 \pm 0.001$ & 2.87 \\
\hline $0.38 \pm 0.01$ & $0.13 \pm 0.00$ & $0.898 \pm 0.003$ & 4.23 \\
\hline $0.51 \pm 0.02$ & $0.45 \pm 0.00$ & $0.915 \pm 0.002$ & 4.87 \\
\hline $0.55 \pm 0.02$ & $0.93 \pm 0.01$ & $0.922 \pm 0.002$ & 5.31 \\
\hline $0.60 \pm 0.03$ & $1.36 \pm 0.01$ & $0.893 \pm 0.003$ & 3.52 \\
\hline $0.54 \pm 0.03$ & $1.91 \pm 0.01$ & $0.858 \pm 0.005$ & 3.29 \\
\hline $0.55 \pm 0.06$ & $2.41 \pm 0.03$ & $0.836 \pm 0.003$ & 3.12 \\
\hline $0.56 \pm 0.06$ & $3.40 \pm 0.02$ & $0.829 \pm 0.002$ & 3.03 \\
\hline $0.92 \pm 0.07$ & $4.01 \pm 0.02$ & $0.916 \pm 0.003$ & 4.45 \\
\hline
\end{tabular}




\section{References}

1. Environment New Zealand 2007, The Ministry for the Environment, New Zealand.

2. Smith, C. M. New Zealand Journal of Marine and Freshwater Research 1987, 21, 627-639.

3. Sumner, M. E. Handbook of Soil Science; CRC Press, 2000.

4. Mortimer, C. H. Limnology and Oceanography 1971, 16, 387-404.

5. Resource Management Act, New Zealand, 1991.

6. Drinking-water Standards for New Zealand 2005; Ministry of Health, Wellington, New Zealand, 2005.

7. The Wellington City Council Trade Waste Bylaw 2004; Wellington City Council, Wellington, New Zealand, 2004.

8. Cameron, D. Moa Point Wastewater Treatment Plant Resource Consent Applications and Assessment of Effects on the Environment; Wellington City Council, Wellington, New Zealand, 2007.

9. Chou, H.-N.; Naleway, C. A. Analytical Chemistry 1984, 56, 1737-1738.

10. Standard Methods for the Examination of Water and Wastewater, 19th ed.; Greenberg, A. E., Clesceri, L. S., Eaton, A. D., Eds.; American Public Health Association: Washington, DC, U. S. A, 1995.

11. Langmuir, I. Journal of the American Chemical Society 1918, 40, 1361-1402.

12. Adamson, A. W. Physical Chemistry of Surfaces; J. Wiley, New York, 1982.

13. Atkins, P. W. Physical Chemistry, 4th ed.; Oxford University Press, 1992.

14. Agyei, N. M.; Strydom, C. A.; Potgieter, J. H. Cement and Concrete Research 2002, 32, 1889-1897.

15. Brunauer, S.; Emmett, P. H.; Teller, E. Journal of the American Chemical Society 1938, 60, 309-319.

16. Marquardt, D. W. Journal of the Society for Industrial and Applied Mathematics 1963, 11, 431-441.

17. Choy, K. K. H.; McKay, G.; Porter, J. F. Resources, Conservation and Recycling 1999, 27, 57-71.

18. Boyd, G. E.; Adamson, A. W.; Myers, L. S. Journal of the American Chemical Society 1947, 69, 2836-2848.

19. Ho, Y. S.; McKay, G. Process Biochemistry 1999, 34, 451-465.

20. Johnston, J. H.; McFarlane, A. J.; Borrmann, T. NZ Patent Specification 537,747, 2006.

21. Johnston, J. H.; McFarlane, A. J.; Borrmann, T.; Moraes, J. Current Applied Physics 2004, 4, 411-414. 
22. McFarlane, A. J. The Synthesis and Characterisation of Nano-structured Calcium Silicate, Ph.D. thesis, Victoria University of Wellington, 2008.

23. Chen, J. J.; Thomas, J. J.; Taylor, H. F. W.; Jennings, H. M. Cement and Concrete Research 2004, 34, 1499-1519.

24. ASTM Standard D281-95 (2007), Standard Test Method for Oil Adsorption of Pigments by Spatula Rub-out, ASTM International, West Conshohocken, PA, U. S. A., 2007.

25. ISO Standard 2470:1999 Paper, board and pulps - Measurement of diffuse blue reflectance factor (ISO brightness), International Organisation for Standardization, Geneva Switzerland, 1999.

26. International Commission on Illumination CIE $1976\left(L^{*}, a^{*}, b^{*}\right)$ color space, Vienna, Austria, 1976.

27. McFarlane, A. J. Victoria University of Wellington, Personal communication, 2008.

28. Borrmann, T.; Johnston, J. H.; McFarlane, A. J.; MacKenzie, K. J. D.; Nukui, A. Powder Diffraction 2008, 23, 204-212.

29. Handbook of Chemistry and Physics, 86th ed.; Lide, D. R., Ed.; CRC Press, 2005.

30. Borrmann, T.; Cairns, M. J.; Anderson, B. G.; Höll, W. H.; Johnston, J. H. International Journal of Environment and Waste Management 2008, in press, .

31. Iler, R. K. The Chemistry of Silica: Solubility, Polymerization, Colloid and Surface Properties, and Biochemistry; Wiley, New York, 1979.

32. Borrmann, T.; Johnston, J. H.; McFarlane, A. J.; Anderson, B. G.; Witkowski, D.; Lim, T. H. Calcium Silicate Facilitated Polymerisation of MAS. ICSM The International Conference on Science and Technology of Synthetic Metals, Trinity College Dublin, Ireland, July 2-7, 2006.

33. Southam, D. C.; Lewis, T. W.; McFarlane, A. J.; Johnston, J. H. Current Applied Physics 2004, 4, 355-358.

34. Southam, D. C. Phosphate in the environment: its analysis and removal using advanced materials, Ph.D. thesis, University of Tasmania, 2005.

35. Southam, D. C.; Lewis, T. W.; McFarlane, A. J.; Borrmann, T.; Johnston, J. H. Journal of Colloid and Interface Science 2008, 319, 489-497.

36. Chiang, C. K.; Fincher, C. R., Jr.; Park, Y. W.; Heeger, A. J.; Shirakawa, H.; Louis, E. J.; Gau, S. C.; MacDiarmid, A. G. Physical Review Letters 1977, 39, 1098-1101.

37. Chiang, C. K.; Dury, M. A.; Gau, S. C.; Heeger, A. J.; Louis, E. J.; MacDiarmid, A. G.; Park, Y. W.; Shirakawa, H. Journal of the American Chemical Society 1978, 100, 1013-1014.

38. Letherberg, H. Journal of the Chemical Society 1862, 15, 161-163.

39. ISO Standard 6878:2004 Water Quality - Determination of phosphorus Ammonium molybdate spectrographic method, International Organisation for Standardization, Geneva, Switzerland, 2004. 
40. Athanasopoulos, N. Flame Methods Manual for Atomic Absorption; GBC Scientific Equipment Pty. Ltd., Dandenong, Vic. Australia, 1989.

41. Powder Diffraction File, Version 4+, International Centre for Diffraction Data, Pennsylvania, U. S. A., 2007.

42. Flowsorb II 2300 Instruction Manual for Determining Single Point and Multipoint Surface Area, Total Pore Volume, and Pore Area and Volume Distribution; Micromeritics Instrument Corporation, Norcross, GA, U. S. A, 1990.

43. Wojdyr, M. fityk Version 0.8.2 http://www.unipress.waw.pl/fityk/ (10 June 2008).

44. Shirley, D. A. Physical Review, B 1972, 5, 4709-4714.

45. Berger, P.; Adelman, N. B.; Beckman, K. J.; Campbell, D. J.; Ellis, A. B.; Lisensky, G. C. Journal of Chemical Education 1999, 76, 943-948.

46. Massart, R. IEEE Transactions on Magnetics 1981, MAG-17, 1247-1248.

47. Borrmann, T.; Dominis, A.; McFarlane, A. J.; Johnston, J. H.; Richardson, M. J.; Kane-Maguire, L. A. P.; Wallace, G. G. Journal of Nanoscience and Nanotechnology 2007, 7, 4303-4310.

48. Duffus, J. H. Pure and Applied Chemistry 2002, 74, 793-807.

49. McNaught, A. D.; Wilkinson, A. Compendium of Chemical Terminology: IUPAC Recommendations, 2nd ed.; Blackwell Science, 1997.

50. Alward, G.; Findlay, T. SI Chemical Data, 5th ed.; J. Wiley \& Sons, Australia, 2002.

51. Näsänen, R.; Tamminen, V. Journal of the American Chemical Society 1949, 71, 1994-1998.

52. Xu, Y.-M.; Wang, R.-S.; Wu, F. Journal of Colloid and Interface Science 1999, 209, 380-385.

53. Salehizadeh, H.; Shojaosadati, S. A. Water Research 2003, 37, 4231-4235.

54. Cowan, J. A. Inorganic Biochemistry. An Introduction, 2nd ed.; Wiley-VCH, Inc., New York, 1997.

55. Kiam, W.; Schwedeski, B. Bioinorganic Chemistry: Inorganic Elements in the Chemistry of Life. An Introduction and Guide; John Wiley and Sons, Chichester, 1994.

56. Copper (II) Sulfate Pentahydrate Safety Data Sheet, Sigma-Aldrich New Zealand Ltd., 2008.

57. Barba, F.; Callejas, P. Journal of Materials Science 2006, 41, 5227-5230.

58. Dobrevsky, I.; Dimova-Todorova, M.; Panayotova, T. Desalination 1997, 108, 277 280.

59. Chmielewski, A. G.; Urbanski, T. S.; Migdal, W. Hydrometallurgy 1997, 45, 333344.

60. Panayotova, T.; Dimova-Todorova, M.; Dobrevsky, I. Desalination 2007, 206, 135140. 
61. Cavaco, S. A.; Fernandes, S.; Quina, M. M.; Ferreira, L. M. Journal of Hazardous Materials 2007, 144, 634-638.

62. Álvarez-Ayuso, E.; García-Sánchez, A.; Querol, X. Water Research 2003, 37, 48554862.

63. Kargman, V. B.; Sokolova, L. P.; Saldadze, G. K.; Beklemisheva, G. S.; Samsonov, A. K. Russian Journal of Applied Chemistry (Translation of Zhurnal Prikladnoi Khimii) 2001, 74, 1858-1863.

64. Huang, H. L.; Wang, H. P.; Wei, G. T.; Sun, I. W.; Huang, J. F.; Yang, Y. W. Environmental Science \& Technology 2006, 40, 4761-4764.

65. Ure, A. M.; Welch, K. H. Journal of the Science of Food and Agriculture 1982, 33, 711-14.

66. Adhoum, N.; Monser, L.; Bellakhal, N.; Belgaied, J.-E. Journal of Hazardous Materials 2004, 112, 207-213.

67. Liu, C.-K.; Li, C.-W. Desalination 2004, 169, 185-192.

68. Hor, Y. P.; Mohamed, N. Journal of Applied Electrochemistry 2003, 33, 279-285.

69. Hor, Y. P.; Mohamed, N. Journal of Applied Electrochemistry 2005, 35, 609-613.

70. Stefanowicz, T.; Osińska, M.; Napieralska-Zagozda, S. Hydrometallurgy 1997, 47, 69-90.

71. Vaaler, L. E. Journal of the Electrochemical Society 1978, 125, 204-209.

72. Kuchar, D.; Fukuta, T.; Onyango, M. S.; Matsuda, H. Journal of Hazardous Materials 2006, 138, 86-94.

73. Nosier, S. A.; Alhamed, Y. A.; Alturaif, H. A. Separation and Purification Technology 2007, 52, 454-460.

74. Ahluwalia, S. S.; Goyal, D. Bioresource Technology 2007, 98, 2243-2257.

75. Cochrane, E. L.; Lu, S.; Gibb, S. W.; Villaescusa, I. Journal of Hazardous Materials 2006, B137, 198-206.

76. Metal Ions in Biological Systems. Volume 1: Simple Complexes; Sigel, H., Ed.; Marcel Dekker Inc, New York, 1974.

77. Muraleedharan, T. R.; Venkobachar, C. Biotechnology and Bioengineering 1990, $35,320-325$.

78. Tsezos, M. Biotechnology and Bioengineering 1983, 25, 2025-2040.

79. Gardea-Torresdey, J. L.; de la Rosa, G.; Peralta-Videa, J. R. Pure and Applied Chemistry 2004, 76, 801-813.

80. Schneider, I. A. H.; Rubio, J.; Smith, R. W. International Journal of Mineral Processing 2001, 62, 111-120.

81. Schneider, I. A. H.; Smith, R. W.; Rubio, J. Minerals Engineering 1999, 12, 255260. 
82. Frank, P.; Benfatto, M.; Szilagyi, R. K.; D’Angelo, P.; Della Longa, S.; Hodgson, K. O. Inorganic Chemistry 2005, 44, 1922-1933.

83. Šljukić, B.; Wildgoose, G. G.; Crossley, A.; Jones, J. H.; Jiang, L.; Jones, T. G. J.; Compton, R. G. Journal of Materials Chemistry 2006, 16, 970-976.

84. Wang, C. C.; Wang, C. C. Journal of Applied Polymer Science 2005, 97, 2457-2468.

85. Kuroda, Y.; Kotani, A.; Maeda, H.; Moriwaki, H.; Morimato, T.; Nagao, M. Journal of the Chemical Society, Faraday Transactions 1992, 88, 1583-1590.

86. Kuroda, Y.; Kotani, A.; Uemura, A.; Yoshikawa, Y.; Morimoto, T. Journal of the Chemical Society, Chemical Communications 1989, 1631-1632.

87. Ursini, O.; Lilla, E.; Montanari, R. Journal of Hazardous Materials 2006, 137, 1079-1088.

88. de Peña, Y. P.; López, W.; Burguera, J. L.; Burguera, M.; Gallignani, M.; Brunetto, R.; Carrero, P.; Rondon, C.; Imbert, F. Analytica Chimica Acta 2000, 403, 249-258.

89. Feng, D.; Aldrich, C.; Tan, H. Hydrometallurgy 2000, 56, 359-368.

90. Choi, J. H.; Kim, S. D.; Kwon, Y. J.; Kim, W. J. Microporous and Mesoporous Materials 2006, 96, 157-167.

91. Kuroda, Y.; Kumashiro, R.; Nagao, M. Applied Surface Science 2002, 196, 408422.

92. Kuroda, Y.; Yagi, K.; Yoshikawa, Y.; Kumashiro, R.; Nagao, M. Chemical Communications 1997, 2241-2242.

93. Bolis, V.; Maggiorini, S.; Meda, L.; D’Acapito, F.; Turnes Palomino, G.; Bordiga, S.; Lamberti, C. Journal of Chemical Physics 2000, 113, 9248-9261.

94. Gougar, M. L. D.; Scheetz, B. E.; Roy, D. M. Waste Management 1996, 16, 295303.

95. Atkins, M.; Glasser, F. P. Waste Management 1992, 12, 105-31.

96. Coleman, N. J.; Lee, W. E.; Slipper, I. J. Journal of Hazardous Materials 2005, 121, 203-213.

97. Pamukoglu, M. Y.; Kargi, F. Journal of Hazardous Materials 2006, 138, 479-484.

98. Ok, Y. S.; Yang, J. E.; Zhang, Y.-S.; Kim, S.-J.; Chung, D.-Y. Journal of Hazardous Materials 2007, 147, 91-96.

99. Pellegrini, F.; Hills, C. D.; Carey, P. J.; Gardner, K. H.; Maries, A. Advances in Applied Ceramics 2006, 105, 185-190.

100. Borrmann, T.; McFarlane, A. J.; Johnston, J. H. Calcium silicate as a matrix for antifouling applications. NZIC Conference, Molecules for Life, Napier, New Zealand, 4-7 Dec, 2001.

101. Bowmaker, G. A. Auckland University, Personal communication, 2008. 
102. Ng, J. C. Y.; Cheung, W. H.; McKay, G. Journal of Colloid and Interface Science 2002, 255, 64-74.

103. Rengaraj, S.; Yeon, J.-W.; Kim, Y.; Jung, Y.; Ha, Y.-K.; Kim, W.-H. Journal of Hazardous Materials 2007, 143, 469-477.

104. Spark, K. M.; Johnson, B. B.; Wells, J. D. Australian Journal of Chemistry 1990, $43,749-754$.

105. Dunlop, D. J.; Özdemir, Ö. Rock magnetism: fundamentals and frontiers, 1st ed.; Cambridge studies in magnetism; Cambridge University Press, 1997.

106. Néel, L. Advances in Physics 1955, 4, 191-243.

107. Yamanaka, T.; Okita, M. Physics and Chemistry of Minerals 2001, 28, 102-109.

108. Svoboda, J. Minerals Engineering 2001, 14, 1493-1503.

109. Watson, J. H. P. Journal of Applied Physics 1973, 44, 4209-13.

110. Moeser, G. D.; Roach, K. A.; Green, W. H.; Hatton, T. A.; Laibinis, P. E. AIChe Journal 2004, 50, 2835-2848.

111. Hubbuch, J. J.; Thomas, O. R. T. Biotechnology and Bioengineering 2002, 79, 301313.

112. Franzreb, M.; Reichert, C. United States Patent Application 2006/0016732, 2006.

113. Meyer, A.; Hansen, D. B.; Gomes, C. S. G.; Hobley, T. J.; Thomas, O. R. T.; Franzreb, M. Biotechnology Progress 2005, 21, 244-254.

114. Rayner, J. G.; Napier-Munn, T. J. Minerals Engineering 2000, 13, 277-285.

115. Priestley, A. J. Australian Patent 553,423, 1982.

116. Pavlova, S.; Dobrevsky, I. Desalination 2005, 173, 55-59.

117. Franzreb, M.; Kampeis, P.; Franz, M.; Eberle, S. H. Acta Hydrochimica et Hydrobiologica 1998, 26, 213-217.

118. Bomatí-Miguel, O.; Mazeina, L.; Navrotsky, A.; Veintemillas-Verdaguer, S. Chemistry of Materials 2008, 20, 591-598.

119. Jones, M., Jr. Organic Chemistry, 2nd ed.; W. W. Norton \& Company, Inc., 2000.

120. Shriver, D. F.; Atkins, P. W. Inorganic Chemistry, 3rd ed.; Oxford University Press, 1999.

121. Frink, C. R. Soil Science Society of America Proceedings 1969, 33, 369-372.

122. Sharpley, A. Phosphorus Availability. In Handbook of Soil Science; Sumner, M. E., Ed., 1st ed.; CRC Press, 2000.

123. Weeramantry, C. G. Nauru: environmental damage under international trusteeship; Oxford University Press, Melbourne, 1992.

124. Kasama, T.; Watanabe, Y.; Yamada, H.; Murakami, T. Applied Clay Science 2004, $25,167-177$. 
125. Georgantas, D. A.; Grigoropoulou, H. P. Journal of Colloid and Interface Science 2007, 315, 70-79.

126. Berkowitz, J.; Anderson, M. A.; Amrhein, C. Water Research 2006, 40, 911-6.

127. Ayoub, G. M.; Koopman, B.; Pandya, N. Water Environment Research 2001, 73, 478-485.

128. Onyango, M. S.; Kuchar, D.; Kubota, M.; Matsuda, H. Industrial \& Engineering Chemistry Research 2007, 46, 894-900.

129. Chen, J.; Kong, H.; Wu, D.; Chen, X.; Zhang, D.; Sun, Z. Journal of Hazardous Materials 2007, 139, 293-300.

130. Song, Y.; Weidler, P. G.; Berg, U.; Nueesch, R.; Donnert, D. Chemosphere 2006, 63, 236-243.

131. Karaca, S.; Gürses, A.; Ejder, M.; Açikyildiz, M. Journal of Colloid and Interface Science 2004, 277, 257-263.

132. Zhang, J.-Z.; Huang, X.-L. Environmental Science \& Technology 2007, 41, 27892795 .

133. Monte, M. B. M.; Duarte, A. C. P.; Bonapace, J. A. P.; Do Amaral, J., Manoel R.; Vieyra, A.; De Souza-Barros, F. Origins of Life and Evolution of the Biosphere 2003, $33,37-52$.

134. Tzou, Y. M.; Wang, M. K.; Loeppert, R. H. Archives of Environmental Contamination and Toxicology 2003, 44, 445-453.

135. Badillo-Almaraz, V. E.; Ly, J. Journal of Colloid and Interface Science 2003, 258, 27-32.

136. Liu, C.; Huang, Y.; Shen, W.; Cui, J. Biomaterials 2001, 22, 301-306.

137. Heughebaert, J. C.; Montel, G. Comptes Rendus des Seances de l'Academie des Sciences, Serie C: Sciences Chimiques 1976, 283, 573-576.

138. Brady, N. C. The Nature and Properties of Soils, 8th ed.; MacMillan Publishing Co. Inc.: New York, 1974.

139. Jaynes, W. F.; Moore, J., P. A.; Miller, D. M. Journal of Environmental Quality 1999, 28, 530-536.

140. Mino, T. Biochemistry (Moscow)(Translation of Biokhimiya (Moscow)) 2000, 65, 341-348.

141. Mulkerrins, D.; Dobson, A. D. W.; Colleran, E. Environment International 2004, 30, 249-259.

142. van Loosdrecht, M. C. M.; Hooijmans, C. M.; Brdjanovic, D.; Heijnen, J. J. Applied Microbiology and Biotechnology 1997, 48, 289-296.

143. You, S. J.; Ouyang, C. F. Journal of Environmental Engineering (Reston, VA, United States) 2005, 131, 883-891.

144. Pierri, E.; Tsamouras, D.; Dalas, E. Journal of Crystal Growth 2000, 213, 93-98. 
145. Ganrot, Z.; Dave, G.; Nilsson, E. Bioresource Technology 2007, 98, 3112-3121.

146. Chimenos, J. M.; I., F. A.; Villalba, G.; Segarra, M.; Urrutiocoecha, A.; Artaza, B.; Espiell, F. Water Research 2003, 57, 1601-1607.

147. Allen, D. G.; Barrow, N. J.; Bolland, M. D. A. Australian Journal of Soil Research 2001, 39, 1433-1442.

148. Bera, R.; Seal, A.; Bhattacharyya, P.; Mukhopadhyay, K.; Giri, R. Environmental Geology 2006, 51, 399-407.

149. Edis, R. B.; Bramley, R. G. V.; White, R. E.; Wood, A. W. Marine and Freshwater Research 2002, 53, 961-970.

150. Bhadoria, P. S.; Steingrobe, B.; Claassen, N.; Liebersbach, H. Plant and Soil 2002, 246, 41-52.

151. Violante, A.; Pigna, M. Soil Science Society of America Journal 2002, 66, 17881796.

152. Van Emmerik, T. J.; Sandstroem, D. E.; Antzutkin, O. N.; Angove, M. J.; Johnson, B. B. Langmuir 2007, 23, 3205-3213.

153. Ler, A.; Stanforth, R. Environmental Science \& Technology 2003, 37, 2694-2700.

154. Mustafa, S.; Zaman, M. I.; Gul, R.; Khan, S. Separation and Purification Technology 2008, 59, 108-114.

155. Khare, N.; Hesterberg, D.; Martin, J. D. Environmental Science \& Technology 2005, $39,2152-2160$.

156. Kostura, B.; Kulveitová, H.; Leško, J. Water Research 2005, 39, 1795-1802.

157. Chubar, N. I.; Kanibolotskyy, V. A.; Strelko, V. V.; Gallios, G. G.; Samanidou, V. F.; Shaposhnikova, T. O.; Milgrandt, V. G.; Zhuravlev, I. Z. Colloids and Surfaces, A: Physicochemical and Engineering Aspects 2005, 255, 55-63.

158. Boujelben, N.; Bouzid, J.; Elouear, Z.; Feki, M.; Jamoussi, F.; Montiel, A. Journal of Hazardous Materials 2008, 151, 103-110.

159. Ruixia, L.; Jinlong, G.; Hongxiao, T. Journal of Colloid and Interface Science 2002, 248, 268-274.

160. Hench, L. L. Journal of the American Ceramic Society 1991, 74, 1487-510.

161. Berg, U.; Donnert, D.; Ehbrecht, A.; Bumiller, W.; Kusche, I.; Weidler, P. G.; Nueesch, R. Colloids and Surfaces, A: Physicochemical and Engineering Aspects 2005, 265, 141-148.

162. Inskeep, W. P.; Silvertooth, J. C. Geochimica et Cosmochimica Acta 1988, 52, 188393.

163. Borrmann, T.; Cairns, M. J.; Johnston, J. H.; Lai, J. C.; Anderson, B. G.; Milestone, N. B.; Höll, W. H. Nanostructured Calcium Silicate as MicroSorbent Material. Nano- \& Bionano-Technology, Quantum Transport \& Quantum Functional Semiconductors (NBT-QT), Wellington, New Zealand, 8-10 Feb, 2007. 
164. Alberto, R. Technetuim. In Comprehensive Coordination Chemistry II: From Biotechnology to Nanotechnology; McCleverty, J. A., Meyer, T. J., Eds.; Elsevier Ltd., 2004; Vol. 5.

165. Liang, L. Y.; Gu, B. H.; Yin, X. P. Separations Technology 1996, 6, 111-122.

166. MacDiarmid, A. G. Angewandte Chemie (International Edition) 2001, 40, 25812590.

167. Jansen, S. A.; Duong, T.; Major, A.; Wei, Y.; Sein, J., Lawrence T. Synthetic Metals 1999, 105, 107-113.

168. Reece, D. A. Development of Conducting Polymers for Separations, Ph.D. thesis, University of Wollongong, 2003.

169. Wei, Y.; Jang, G.-W.; Chan, C.-C.; Hsueh, K. F.; Hariharan, R.; Patel, S. A.; Whitecar, C. K. Journal of Physical Chemistry 1990, 94, 7716-7721.

170. Venancio, E. C.; Wang, P.-C.; MacDiarmid, A. G. Synthetic Metals 2006, 156, 357.

171. Li, X.; Dai, N.; Wang, G.; Song, X. Journal of Applied Polymer Science 2008, 107, 403-408.

172. Liu, Y.; Liu, P.; Su, Z. Synthetic Metals 2007, 157, 585-591.

173. Tang, Q.; Wu, J.; Ao, H.; Sun, H.; Lin, L.; Lin, J. Polymers \& Polymer Composites 2007, 15, 605-610.

174. Wu, C.-S.; Huang, Y.-J.; Hsieh, T.-H.; Huang, P.-T.; Hsieh, B.-Z.; Han, Y.-K.; Ho, K.-S. Journal of Polymer Science, Part A: Polymer Chemistry 2008, 46, 18001809.

175. Kloprogge, J. T.; Duong, L. V.; Frost, R. L. Environmental Geology 2005, 47, 967981.

176. Borrmann, T.; McFarlane, A. J.; Johnston, J. H.; Wallace, G. G. Synthesis of conducting polymers in the presence of novel calcium silicate. AMN-1 International Conference on Advanced Materials and Nanotechnology, Wellington, New Zealand, Feb., 2003.

177. Abram, U. Rhenium. In Comprehensive Coordination Chemistry II: From Biotechnology to Nanotechnology; McCleverty, J. A., Meyer, T. J., Eds.; Elsevier Ltd., 2004; Vol. 5.

178. Mujica, C.; Llanos, J.; Peters, K.; Peters, E.-M.; von Schnering, H. G. Boletín de la Sociedad Chilena de Química 2000, 45, 329-332.

179. Ohlhausen, L.; Cockrum, D.; Register, J.; Roberts, K.; Long, G. J.; Powell, G. L.; Hutchinson, B. B. Inorganic Chemistry 1990, 29, 4886-4891.

180. Jafarifar, D.; Daryanavard, M. R.; Sheibani, S. Hydrometallurgy 2005, 78, 166-171.

181. Okal, J. Applied Catalysis, A: General 2005, 287, 214-220.

182. Gholamkhass, B.; Mametsuka, H.; Koike, K.; Tanabe, T.; Furue, M.; Ishitani, O. Inorganic Chemistry 2005, 44, 2326-2336. 
183. Shariat, M. H.; Hassani, M. Journal of Materials Processing Technology 1998, 74, 243-250.

184. Mozammel, M.; Sadrnezhaad, S. K.; Badami, E.; Ahmadi, E. Hydrometallurgy 2007, 85, 17-23.

185. Kholmogorov, A. G.; Kononova, O. N.; Kachin, S. V.; Ilyichev, S. N.; Kryuchkov, V. V.; Kalyakina, O. P.; Pashkov, G. L. Hydrometallurgy 1999, 51, 1935.

186. Szabó, S.; Bakos, I. Journal of Electroanalytical Chemistry 2000, 492, 103.

187. Zagorodnyaya, A. N.; Abisheva, Z. S. Hydrometallurgy 2002, 65, 69-76.

188. Kim, E.; Benedetti, M. F.; Boulegue, J. Water Research 2004, 38, 448-454.

189. Smith, J., William T.; Maxwell, G. E. Journal of the American Chemical Society 1951, 73, 658-60.

190. Kalyakina, O. P.; Kononova, O. N.; Kachin, S. V.; Kholmogorov, A. G. Bulletin of the Korean Chemical Society 2004, 25, 79-84.

191. Lan, X.; Liang, S.; Song, Y. Hydrometallurgy 2006, 82, 133-136.

192. Schrebler, R.; Cury, P.; Suárez, C.; Muñoz, E.; Vera, F.; Córdova, R.; Gómez, H.; Ramos-Barrado, J. R.; Leinen, D.; Dalchiele, E. A. Thin Solid Films 2005, 483, 50.

193. Hahn, B. P.; May, R. A.; Stevenson, K. J. Langmuir 2007, 23, 10837-10845.

194. Zemskova, L. A.; Voit, A. V.; Nikolenko, Y. M.; Sergienko, V. I.; Troshkina, I. D.; Plevaka, A. V.; Maiboroda, S. B.; Chekmarev, A. M. Journal of Nuclear and Radiochemical Sciences 2005, 6, 221-222.

195. Xiong, C.; Yao, C.; Wu, X. Hydrometallurgy 2008, 90, 221-226.

196. McDonald, F. E.; Towne, T. B.; Schultz, C. C. Pure and Applied Chemistry 1998, $70,355-358$.

197. Guhlke, S.; Beets, A. L.; Oetjen, K.; Mirzadeh, S.; Biersack, H. J.; Knapp, F. F., Jr. The Journal of Nuclear Medicine 2000, 41, 1271-1278.

198. Petrov, K. I.; Rubtsov, M. V.; Sinitsyn, N. M.; Travkin, V. F. Russian Journal of Inorganic Chemistry 1969, 14, 1704-1707.

199. Pourbaix, M.; de Zoubov, N. Rhenium. In Atlas of Electrochemical Equilibria in Aqueous Solutions; Pourbaix, M., Ed.; Pergamon Press, 1966.

200. Wehrle, B.; Limbach, H.-H.; Mortensen, J.; Heinze, J. Angewandte Chemie 1989, $101,1781-3$.

201. Black, L.; Garbev, K.; Stemmermann, P.; Hallam, K. R.; Allen, G. C. Cement and Concrete Research 2003, 33, 899-911.

202. Black, L.; Garbev, K.; Beuchle, G.; Stemmermann, P.; Schild, D. Cement and Concrete Research 2006, 36, 1023-1031.

203. Moulder, J. F.; Stickle, W. F.; Sobol, P. F.; Bomben, K. D. Handbook of X-ray Photoelectron Spectroscopy; Perkin-Elmer Co, Minnesota, U. S. A., 1992. 
204. Busey, R. H.; Sprague, E. D.; Bevan, R. B., Jr. Journal of Physical Chemistry 1969, 73, 1039-1042.

205. Soto, G.; Rosas, A.; Farias, M. H.; De la Cruz, W.; Diaz, J. A. Materials Characterization 2007, 58, 519-526.

206. Tisley, D. G.; Walton, R. A. Inorganic Chemistry 1973, 12, 373-8.

207. Chan, S. F.; Tan, G. S. Journal of Inorganic and Nuclear Chemistry 1976, 38, 345-6.

208. Schmid, S.; Straehle, J. Zeitschrift für Naturforschung, B: Chemical Sciences 1991, $46,235-44$.

209. Hirao, T. Coordination Chemistry Reviews 2002, 226, 81-91.

210. Johnson, J. W.; Brody, J. F.; Ansell, G. B.; Zentz, S. Inorganic Chemistry 1984, 23, 2415-18.

211. Méndez, E.; Cerdá, M. F.; Castro Luna, A. M.; Zinola, C. F.; Kremer, C.; Martins, M. E. Journal of Colloid and Interface Science 2003, 263, 119-132. 